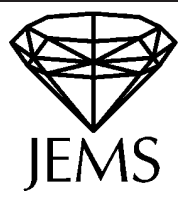

\title{
The $n$-centre problem of celestial mechanics for large energies
}

Received October 16, 2000 / final version received June 18, 2001

Published online August 15, 2001 - (C) Springer-Verlag \& EMS 2001

\begin{abstract}
We consider the classical three-dimensional motion in a potential which is the sum of $n$ attracting or repelling Coulombic potentials. Assuming a non-collinear configuration of the $n$ centres, we find a universal behaviour for all energies $E$ above a positive threshold. Whereas for $n=1$ there are no bounded orbits, and for $n=2$ there is just one closed orbit, for $n \geq 3$ the bounded orbits form a Cantor set. We analyze the symbolic dynamics and estimate Hausdorff dimension and topological entropy of this hyperbolic set. Then we set up scattering theory, including symbolic dynamics of the scattering orbits and differential cross section estimates. The theory includes the $n$-centre problem of celestial mechanics, and prepares for a geometric understanding of a class of restricted $n$-body problems. To allow for applications in semiclassical molecular scattering, we include an additional smooth (electronic) potential which is arbitrary except its Coulombic decay at infinity. Up to a (optimal) relative error of order $1 / E$, all estimates are independent of that potential but only depend on the relative positions and strengths of the centres. Finally we show that different, non-universal, phenomena occur for collinear configurations.
\end{abstract}

\section{Contents}

1 Introduction .......................... 2

2 Basic definitions . . . . . . . . . . . . . . . . . . . . . . 8

3 The Kustaanheimo-Stiefel transformation . . . . . . . . . . . . . . . . . 12

4 Application of the KS-transformation . . . . . . . . . . . . . . . . 15

5 Regularization by phase space extension . . . . . . . . . . . . . . . 26

6 Møller transformations ...................... . . 30

7 The flow between near-collisions . . . . . . . . . . . . . . . . . . 40

8 The single scattering process ................. . . . 42

9 Long paths within the interaction zone . . . . . . . . . . . . . . . . . . . . 49

10 The Poincaré map . . . . . . . . . . . . . . . . . . . . 57

11 Existence of an invariant cone field . . . . . . . . . . . . . . 63

12 Symbolic dynamics . . . . . . . . . . . . . . . . . . . . . . . 70

13 Fractal dimension . . . . . . . . . . . . . . . . . . . 76

14 Topological entropy . . . . . . . . . . . . . . . . . . . . . 86

A. Knauf: Mathematisches Institut, Universität Erlangen-Nürnberg, Bismarckstrasse 1 1/2, 91054 Erlangen, Germany; e-mail: knauf@mi.uni-erlangen.de

Mathematics Subject Classification (2000): 58F12, 70D05, 70F10, 70H05 


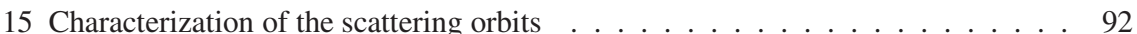

16 The differential cross section . . . . . . . . . . . . . . . . . . . 100

17 The collinear case . . . . . . . . . . . . . . . . . . . . . . . 106

A Aspects of geometry and global analysis . . . . . . . . . . . . . . . . 108

B The two-centre problem . . . . . . . . . . . . . . . . . . . . . . . . 110

References . . . . . . . . . . . . . . . . . . . . . . . 112

\section{Introduction}

The $n$-body problem of celestial mechanics is the problem of solving the Hamilton equation for the Hamiltonian function

$$
H: T^{*} M \rightarrow \mathbb{R} \quad, \quad H\left(\vec{p}_{1}, \ldots, \vec{p}_{n}, \vec{q}_{1}, \ldots, \vec{q}_{n}\right):=\sum_{i=1}^{n} \frac{\vec{p}_{i}^{2}}{2 m_{i}}+v \sum_{i \neq k} \frac{Z_{i} Z_{k}}{\left|\vec{q}_{i}-\vec{q}_{k}\right|}
$$

on the phase space $T^{*} M$ over the configuration space

$$
M:=\left\{\left(\vec{q}_{1}, \ldots, \vec{q}_{n}\right) \in\left(\mathbb{R}^{3}\right)^{n} \mid \vec{q}_{i} \neq \vec{q}_{k} \text { for } i \neq k\right\} .
$$

In celestial mechanics $v=-1$ and the $Z_{i}$ coincide with the positive masses $m_{i}>0$ (in units where the gravitational constant equals one). However in an electrostatical context $v=+1$, the $Z_{i}$ are interpreted as charges and may be positive or negative.

Whereas the one-body problem corresponds to free motion and the two-body problem was solved by Newton, the $n \geq 3$-body problem is analytically nonintegrable.

If one of $n+1$ bodies is much faster than the others then one may approximate its motion by considering the $n$-centre problem. There the Hamiltonian function $\hat{H}: T^{*} \hat{M} \rightarrow \mathbb{R}$ on the phase space $T^{*} \hat{M}$ over the configuration space

$$
\hat{M}:=\mathbb{R}^{3} \backslash\left\{\vec{s}_{1}, \ldots, \vec{s}_{n}\right\}
$$

of that body is given by

$$
\hat{H}(\vec{p}, \vec{q}):=\frac{1}{2} \vec{p}^{2}+V(\vec{q}) \quad \text { with } \quad V(\vec{q}):=-\sum_{l=1}^{n} \frac{Z_{l}}{\left|\vec{q}-\vec{s}_{l}\right|} .
$$

The 2-body problem thus reduces after separation of the centre of mass motion to the 1 -centre problem, that is the Kepler problem.

Moreover, the 2-centre problem is integrable and has been solved by Jacobi (see Appendix B). This solution is particularly relevant for calculating the motion of artificial satellites, since the gravitational field of the earth can be approximated by the one of two centres (analytically continued, since the earth is oblate and not prolate). This has been used by Vinti in [Vi]. See [GKM] for an application.

However, like the $n$-body problem, the $n$-centre problem for $n \geq 3$ is analytically non-integrable, see Bolotin [Bo].

The analogy between the Coulombic and the gravitational interaction is not perfect, since in electrostatics one has repulsive as well as attractive forces. This allows us to approximate molecules by static configurations of nuclei surrounded 
by an electronic cloud of opposite charge. Thus the electronic potential $V$ of the molecule has the form

$$
V(\vec{q})=\sum_{l=1}^{n} \frac{-Z_{l}}{\left|\vec{q}-\vec{s}_{l}\right|}+W(\vec{q}),
$$

$\vec{s}_{l} \in \mathbb{R}^{3}$ being the position, and $-Z_{l} \neq 0$ the charge of the $l$ th nucleus, multiplied by the test charge. The smooth electronic potential $W: \mathbb{R}^{3} \rightarrow \mathbb{R}$ may partially shield the nucleonic charge. We model this by assuming the existence of a net asymptotic charge $Z_{\infty} \in \mathbb{R}$ with

$$
V(\vec{q})=\frac{-Z_{\infty}}{|\vec{q}|}+\mathcal{O}\left(|\vec{q}|^{-1-\epsilon}\right) \quad(\vec{q} \rightarrow \infty) .
$$

Thus we consider the generalized $n$-centre problem (1.1) with these Coulombic potentials $V$.

The understanding of the motion in an $n$-centre potential, $n \geq 3$, is very limited if one considers negative energies $E$. There one expects a complicated mixture of ergodic components and motion on KAM tori.

However, by combining known techniques of celestial mechanics, we show in this article that the high energy motion allows for a more or less complete qualitative and even quantitative description.

Up to an error of relative order $1 / E$, the quantitative aspects treated here do not depend on the precise form of the smooth potential $W$ but only on the charges $Z_{l}$ and positions $\vec{s}_{l}$ of the nuclei.

The qualitative structures do not even depend on these data but only on the number $n$ of nuclei. Thus the case $n=1$ of an atom resembles the Rutherford case, whereas the $n=2$-atomic molecule is similar to the two-centre problem solved by Jacobi. Here we are mainly interested in the case $n \geq 3$ where the dynamics is no longer analytically integrable.

One motivation for this work is to establish the basis for a geometric understanding of certain restricted $(n+1)$-body problems of celestial mechanics, where one is interested in the motion of a fast test body in the force field of $n$ bodies, whose motion is assumed to be known.

Such an analysis should be based on perturbation theory for the $n$-centre problem. In a joint work [DK] with T. Dierkes, we intend to show that indeed the (2-dim.) $n$-centre problem is structurally stable in the following sense. For a sufficiently small local perturbation there exists a homeomorphism conjugating the two phase portraits and leaving the asymptotic initial and final directions of the scattering orbits invariant. This homeomorphism is unique up to changes in the flow direction.

Survey of results. We now describe the techniques and results of this paper.

The class of potentials $V$ under consideration is introduced, together with other basic definitions, in Sect. 2. An important consequence of the fall-off of $V$ is the existence of an interaction zone $\mathcal{I Z}$, a ball in configuration space which contains the points $\vec{s}_{l}$ and which, once left, cannot be reentered by an orbit, see 
(2.17). In Definition 2.4 we formulate the standing assumption that the centres are non-collinear, i.e. no three centres are on a line.

If $V$ contains a negative singularity $\left(Z_{l}>0\right.$ for some $\left.l\right)$, then the Hamiltonian flow generated by (1.1) is incomplete. In the Kepler problem it is well-known that the only sensible way to continue a collision orbit is to reflect it at the singularity in configuration space, for this is the limit behaviour for the Keplerian conic sections in the limit of vanishing angular momentum.

But for our purposes we need to control smoothness and energy dependence of the resulting flow, for potentials of the form (1.2). Due to the singularity at $\vec{s}_{l}$ the usual comparison techniques for o.d.e. are not effective for proving such a result directly.

Instead, near $\vec{s}_{l}$ we apply in Sect. 3 the so-called Kustaanheimo-Stiefel transformation. The Hopf map

$$
\mathbb{C}^{2} \rightarrow \mathbb{R}^{3}, \quad z \mapsto\left(\begin{array}{c}
\left\langle z, \sigma_{1} z\right\rangle \\
\left\langle z, \sigma_{2} z\right\rangle \\
\left\langle z, \sigma_{3} z\right\rangle
\end{array}\right)
$$

with the Pauli matrices $\sigma_{l}$, extends the Hopf fibration $S^{3} \rightarrow S^{2}$. A cognate map of the phase spaces (cotangent bundles) is known [KS,StS] as the KustaanheimoStiefel $(K S)$ transformation. It relates the positive energy Keplerian dynamics with the one of a particle in an inverted harmonic potential.

The KS transformation was used in celestial mechanics (see, e.g. Aarseth and Mikkola [AaMi] and articles by Aarseth and by Heggie cited therein), applied to spectral problems of Schrödinger operators (see, e.g. [HKSW]), and shown in [GK] to regularize the semiclassical dynamics.

The perturbation estimates of Sect. 4 work in the covering phase space of the KS transformation.

Although the leading contribution to $V$ near $\vec{s}_{l}$ is the Kepler potential

$$
-\frac{Z_{l}}{\left|\vec{q}-\vec{s}_{l}\right|}
$$

the following argument indicates that we cannot simply approximate a collision orbit of (1.1) by the Kepler hyperbola for the potential (1.4) which has the same initial conditions $\left(\vec{p}_{0}, \vec{q}_{0}\right)$.

The effective scattering region of (1.4) is a ball of radius $\mathcal{O}(1 / E)$ around $\vec{s}_{l}$, see Lemma 9.1. On the other hand, the time the particle needs to reach the singularity at $\vec{s}_{l}$ from its initial position $\vec{q}_{0}$ is of order $1 / \sqrt{E}$.

Due to the (bounded) difference

$$
\nabla\left[V(\vec{q})-\frac{-Z_{l}}{\left|\vec{q}-\vec{s}_{l}\right|}\right]
$$

of forces exerted on the two particles, within this time the distance between the true orbit and the Kepler orbit can grow to something of the order $1 / E$. So by the above the Kepler orbit starting at $\left(\vec{p}_{0}, \vec{q}_{0}\right)$ may miss the effective scattering region.

But this means that shortly after collision the distance between the two trajectories does not necessarily decrease as $E \nearrow \infty$. 
So instead we effectively show that for every orbit with initial condition $\left(\vec{p}_{0}, \vec{q}_{0}\right)$ there is a Kepler orbit, whose initial condition differs only by $\mathcal{O}(1 / E)$ (in a natural metric) from $\left(\vec{p}_{0}, \vec{q}_{0}\right)$, and which remains in a $\mathcal{O}(1 / E)$-neighbourhood through the whole collision process. In Proposition 4.1 the corresponding statements about perturbations of the inverted harmonic oscillator are formulated. More precisely, it is shown that these perturbed solutions are $C^{1}$-near to the ones of the harmonic oscillator.

Moreover the true scattering process is approximated by Kepler scattering in the $C^{1}$ sense, so that we may use the Kepler solutions if we want to linearize the true flow (see Proposition 8.6).

Whereas the KS transformation is particularly suited to understand the topological and geometrical structures, its disadvantages consist in its local nature, the introduction of a higher dimensional phase space and the reparameterization of time. The first problem, the locality near one singularity, should not be insurmountable. In fact Helffer and Siedentop found in [HS] a generalization of the $\mathrm{KS}$ transformation to two centres.

Time reparameterization, however, is unwanted since we are not only interested in the orbits but also in quantities like the time delay of scattering orbits. Thus we describe in Sect. 5 another regularization method. In Theorem 5.1 we show that the incomplete Hamiltonian system

$$
\left(T^{*} \hat{M}, \omega_{0}, \hat{H}\right)
$$

may be uniquely extended to a smooth complete Hamiltonian system

$$
(P, \omega, H)
$$

whose phase space $P \supset T^{*} \hat{M}$ is a smooth six-dimensional manifold, $\omega$ is a smooth symplectic two-form on $P$ restricting to the canonical symplectic two-form $\omega_{0}=$ $\sum_{i=1}^{3} d q_{i} \wedge d p_{i}$ on $T^{*} \hat{M}$, and $H: P \rightarrow \mathbb{R}$ is a smooth Hamiltonian function with $H \uparrow_{T^{*} \hat{M}}=\hat{H}$.

In fact we linearize the flow near each negative singularity and then add a copy of $\mathbb{R} \times S^{2}$ to $T^{*} \hat{M}$. Here $\mathbb{R}$ parametrizes the energy and $S^{2}$ the incoming (and outgoing) direction of the collision orbit.

Thus we may henceforth work with the complete smooth flow

$$
\Phi: \mathbb{R} \times P \rightarrow P \quad(t \in \mathbb{R})
$$

generated by $H$.

By Theorem 5.1 we may think of the Coulomb singularity as an artefact of the use of the inappropriate phase space coordinates $(\vec{p}, \vec{q})$ which, however, leads to a non-trivial topology of $P$.

In Sect. 6 we introduce the Møller transformation by comparing the flow $\Phi^{t}$ on $P$ with the flow $\Phi_{\infty}^{t}: P_{\infty} \rightarrow P_{\infty}$ generated by the Kepler Hamiltonian function

$$
\hat{H}_{\infty}(\vec{p}, \vec{q}):=\frac{1}{2} \vec{p}^{2}-\frac{Z_{\infty}}{|\vec{q}|}
$$

on its phase space $P_{\infty}$, with $Z_{\infty} \in \mathbb{R}$ defined by (1.3). 
The Mфller transformations are then given by

$$
\Omega^{ \pm}:=\lim _{t \rightarrow \pm \infty} \Phi^{-t} \circ \operatorname{Id} \circ \Phi_{\infty}^{t}
$$

where Id canonically identifies the two phase spaces outside a region near the singularities.

The $\Omega^{ \pm}$are continuous and, under mild conditions on $V$ near infinity, smooth canonical transformations, see Theorem 6.3 and 6.5. So the flow is asymptotically complete, see Corollary 6.4 .

However, we are not primarily interested in these typical properties, but in the specific traits of the multi-scattering dynamics.

It is a general fact that to a large extend the unbounded motion, i.e., scattering is determined by the bounded orbits of positive energy. We denote by $b_{E}$ the set of such bound states of energy $E$.

To control these orbits, we combine the above perturbative results for the single scattering process with an analysis of the motion inside the interaction zone $\mathcal{I Z}$, but away from the singularities (Sect. 7). This turns out to be a $C^{1}$-perturbation of relative order $\mathcal{O}(1 / E)$ of the free motion. Using this result and the one of Sect. 8, we may approximate the true dynamics by a combination of free and of Keplerian motion.

In Sect. 8 the perturbative results of Sect. 4 for the flow in KS space are used to obtain Proposition 8.6 which says that the single scattering process is $C^{1}$-near to pure Kepler scattering, up to a relative error $\mathcal{O}(1 / E)$.

Now in Sect. 9 it is shown that if a trajectory is not strongly reflected in uniformly bounded time by the singularities, it soon leaves the interaction zone (Proposition 9.2).

The bound states are then analyzed in Sect. 10 by a Poincaré section technique. We erect Poincaré surfaces which, in their projections to configuration space, sit between pairs of singularities and then define in (10.4) a Poincaré map $\mathcal{P}_{E}$.

In Proposition 11.2 of Sect. 11 we estimate the linearization of $\mathcal{P}_{E}$, which, up to a relative error $\mathcal{O}(1 / E)$, only depends on the scattering angle at $\vec{s}_{l}$ and the charge $Z_{l}$.

This allows us to establish in Sect. 12, Theorem 12.8 a symbolic dynamics for $b_{E}, E>E_{\mathrm{th}}$. The bounded orbits are described by their sequence of nearcollisions, (which is well defined, since we assumed the configuration of centres to be non-collinear).

Thus for $n=1$ there are no bounded orbits, for $n=2$ the set $b_{E}$ consists of one closed orbit, which is closed. For the case $n \geq 3$ of primary interest, $b_{E}$ is locally homeomorphic to the product of a Cantor set and an interval.

All bounded orbits are hyperbolic. $b_{E}$ has measure zero w.r.t. Liouville measure on the energy shell. The Morse index of a periodic trajectory equals its number of near-collisions.

In Sect. 13 the fractal dimension of this set $b_{E}$ of bounded orbits is estimated. More precisely, we consider its Hausdorff dimension $\operatorname{dim}_{\mathcal{H}}$ and its upper box 
counting dimension $\operatorname{dim}_{\mathcal{B}}$, since it is known that most other definitions lead to numerical values between these two.

Theorem 13.5 says that for energies $E$ above the threshold $E_{\text {th }}$ and $n \geq 3$ centres

$$
\begin{aligned}
1+2 d(E) \cdot\left(1-\mathcal{O}\left((E \ln E)^{-1}\right)\right) \leq \operatorname{dim}_{\mathcal{H}}\left(b_{E}\right) \leq \\
\leq \operatorname{dim}_{\mathcal{B}}\left(b_{E}\right) \leq 1+2 d(E) \cdot\left(1+\mathcal{O}\left((E \ln E)^{-1}\right)\right),
\end{aligned}
$$

with the solution $d(E)$ of a finite matrix eigenvalue problem (13.10). In particular they meet the rough estimate

$$
\operatorname{dim}_{\mathcal{H}}\left(b_{E}\right)=1+\frac{2 \ln (n-1)}{\ln (E)}+\mathcal{O}\left((\ln E)^{-2}\right)=\operatorname{dim}_{\mathcal{B}}\left(b_{E}\right)
$$

In Sect. 14 the topological entropy of the flow $\Phi_{E}^{t}$ of energy $E$ is estimated.

Topological entropy is a quantity which, roughly speaking, measures the information loss per time unit about the state of the system. Here for positive energy $E$ the energy shell $\Sigma_{E}$ is non-compact, so that we use Bowen's definition of entropy. Proposition 14.3 states that

$$
h_{\text {top }}\left(\Phi_{E}^{1}\right)=h_{\text {top }}\left(\Phi_{E}^{1} \uparrow_{b_{E}}\right)
$$

i.e. that the source of information loss is the intricate structure of the set $b_{E}$ of bounded orbits, whereas the scattering orbits only have a sub-exponential dependence on initial conditions.

So the estimates in the proof of Theorem 14.4 can be based on symbolic dynamics. It states that for $E$ large $h_{\text {top }}\left(\Phi_{E}^{1}\right)=0$ for $n=1$ or 2 centres, whereas for $n \geq 3$

$$
h_{\text {top }}\left(\Phi_{E}^{1}\right)=h_{\text {top }}^{\infty} \cdot \sqrt{2 E} \cdot\left(1+\frac{\ln (E)}{E} C_{h_{\text {top }}}+\mathcal{O}(1 / E)\right) .
$$

Here the constants $h_{\text {top }}^{\infty}$ and $C_{h_{\text {top }}}$ are determined by solving a finite matrix eigenvalue problem.

Whereas the factor $\sqrt{2 E}$ is of kinematical nature, in the simplest case of an equilateral triangle $(n=3)$ resp. tetrahedron $(n=4)$ of side length $d$ the constant $h_{\text {top }}^{\infty}$ equals $\ln (n-1) / d$.

This divergence of topological entropy is not in contradiction with integrability (in the sense of independent $C^{\infty}$ integrals of motion) of the hamiltonian dynamics above the energy threshold (compare with Bolsinov and Taimanov [BT]).

Section 15 is devoted to the classification of the scattering orbits. After excluding asymptotic directions in cones of aperture $\mathcal{O}(1 / \sqrt{E})$ around the axes through two nuclei, and near the forward direction, one obtains such a universal classification. Theorem 15.3 states that the orbits of given energy and asymptotic directions are enumerated by the succession of the nuclei they visit. In particular they form a Cantor set if $n \geq 3$. 
The differential cross section $\frac{d \sigma}{d \hat{\theta}^{+}}\left(E, \hat{\theta}^{-}, \hat{\theta}^{+}\right)$of the scattering process is analyzed in Sect. 16. Roughly speaking, this experimentally accessible quantity is the 'probability' that a particle of energy $E$ and initial direction $\hat{\theta}^{-}$has final direction $\hat{\theta}^{+}$. For general potential scattering in $\mathbb{R}^{d}$ one cannot even expect the cross section measure on $S^{d-1} \backslash \hat{\theta}^{-}$(defined in Definition 16.2) to be absolutely continuous w.r.t. Haar measure, see [Kn3]. Here, however, this is the case for energies $E>E_{\mathrm{th}}$, and $\frac{d \sigma}{d \hat{\theta}^{+}}\left(E, \hat{\theta}^{-}, \hat{\theta}^{+}\right)$is defined as the Radon-Nikodym derivative.

Moreover (after excluding cones of $E$-independent aperture) by Theorem 16.4 it differs from the well-known Rutherford cross section in $\mathbb{R}^{d}$

$$
\left(\frac{d \sigma}{d \hat{\theta}^{+}}\left(E, \hat{\theta}^{-}, \hat{\theta}^{+}\right)\right)_{\mathrm{Ru}}=\left(\frac{Z}{4 E \sin ^{2}\left(\frac{1}{2} \Delta \theta\right)}\right)^{d-1}
$$

of the single Coulomb potential with charge $Z=\sqrt{\sum_{l=1}^{n} Z_{l}^{2}}$ only by

$$
\frac{d \sigma}{d \hat{\theta}^{+}}\left(E, \hat{\theta}^{-}, \hat{\theta}^{+}\right)=\left(\frac{d \sigma}{d \hat{\theta}^{+}}\left(E, \hat{\theta}^{-}, \hat{\theta}^{+}\right)\right)_{\mathrm{Ru}} \cdot(1+\mathcal{O}(1 / E)) .
$$

So for these asymptotic data the intricate structure of the scattering orbits is not showing up in the cross section. In fact, Theorem 16.4 also states that the differential cross section is approximated by the Rutherford cross section outside the (much smaller) cones of aperture $\mathcal{O}(1 / \sqrt{E})$, though with less accuracy.

In the final Sect. 17 we show by counterexamples that many results of the paper do not generalize if we drop the assumption of non-collinearity.

The first Appendix is devoted to a comparison between the two-dim. case treated in $[\mathrm{KK}]$ and the three-dim. situation of the present paper. Basically, the analysis of $[\mathrm{KK}]$ is the one of geodesic motion on smooth many-handled surfaces of negative curvature, whereas here we apply perturbation techniques around infinite energy.

In the second Appendix we describe the (known) solution of the purely Coulombic two-centre problem, and its bifurcation diagramme.

Notation. Transposed vectors are used according to typographical, not mathematical needs.

\section{Basic definitions}

We consider the time evolution generated by a Hamiltonian function

$$
\hat{H}(\vec{p}, \vec{q}):=\frac{1}{2} \vec{p}^{2}+V(\vec{q})
$$

with $n$ Coulombic singularities of the potential $V$ situated at the points

$$
\vec{s}_{1}, \ldots, \vec{s}_{n} \in \mathbb{R}_{\vec{q}}^{3} \quad\left(\vec{s}_{i} \neq \vec{s}_{k} \text { for } i \neq k\right) .
$$

To control the asymptotic behaviour, we assume that $V$ decomposes into the sum of a purely Coulombic potential and a short range potential. By this we mean the following: 
Definition 2.1. A smooth, real-valued function $V$ on the configuration space

$$
\hat{M}:=\mathbb{R}_{\vec{q}}^{3} \backslash\left\{\vec{s}_{1}, \ldots, \vec{s}_{n}\right\}
$$

is called Coulombic if

1. There exist $Z_{l} \neq 0, l \in\{1, \ldots, n\}$, such that

$$
V(\vec{q})=\sum_{l=1}^{n} \frac{-Z_{l}}{\left|\vec{q}-\vec{s}_{l}\right|}+W(\vec{q}) \quad(\vec{q} \in \hat{M})
$$

with $W: \mathbb{R}_{\vec{q}}^{3} \rightarrow \mathbb{R}$ smooth. $Z_{l}$ is called the charge of the $l$ th nucleus, and we set

$$
Z_{\max }:=\max \left\{\left|Z_{1}\right|, \ldots,\left|Z_{n}\right|\right\}
$$

2. The potential vanishes at infinity, i.e.

$$
\lim _{|\vec{q}| \rightarrow \infty} V(\vec{q})=0
$$

and there exist $Z_{\infty} \in \mathbb{R}$, called the asymptotic charge, $\epsilon \in(0,1]$ and

$$
R_{\min }>2 \max \left(\left|\vec{s}_{1}\right|, \ldots,\left|\vec{s}_{n}\right|\right)
$$

such that for some $C_{1}>0$

$$
\left|\nabla V(\vec{q})-Z_{\infty} \frac{\vec{q}}{|\vec{q}|^{3}}\right|<\frac{C_{1} \cdot R_{\min }}{|\vec{q}|^{2+\epsilon}} \quad\left(|\vec{q}| \geq R_{\min }\right)
$$

and

$$
\left|\nabla V\left(\vec{q}_{1}\right)-\nabla V\left(\vec{q}_{2}\right)\right|<C_{1} \frac{\left|\vec{q}_{1}-\vec{q}_{2}\right|}{\min \left(\left|\vec{q}_{1}\right|,\left|\vec{q}_{2}\right|\right)^{2+\epsilon}} \quad\left(\left|\vec{q}_{1}\right|,\left|\vec{q}_{2}\right| \geq R_{\min }\right) .
$$

Example 2.2. For the class of purely Coulombic potentials

$$
V(\vec{q}):=\sum_{l=1}^{n} \frac{-Z_{l}}{\left|\vec{q}-\vec{s}_{l}\right|},
$$

the asymptotic charge $Z_{\infty}=\sum_{l=1}^{n} Z_{l}$. Here $V$ meets (2.5) and (2.6) with $\epsilon=1$ (and $C_{1}=\frac{17}{2} n Z_{\max }$ ).

The question of dynamics in a Coulombic potential is called the $n$-centre problem of classical mechanics. 
Remarks 2.3. 1) For $Z_{l}>0$ and $W=0$ this corresponds to the idealization of a celestial body in the force field of $n$ other bodies of masses $Z_{l}$ with fixed positions $\vec{s}_{l}$.

But our definition also covers the physical situation of (classical) scattering by the potential of partially ionized, neutral $\left(Z_{\infty}=0\right)$ or negatively charged quantum molecules. There $Z_{\infty}$ does not coincide with $\sum_{l=1}^{n} Z_{l}$. Again, one expects potentials with $\epsilon=1$, due to exponential decay of the quantum mechanical eigenfunctions of the bound electrons, which in turn leads to an electronic charge distribution which decays exponentially (see Agmon [Ag]).

2) Note that $V(\vec{q})$ and the asymptotic potential $-Z_{\infty} /|\vec{q}|$ appear symmetrically in (2.5), and that (2.4) and (2.6) are met by the asymptotic potential (the last one even with $\epsilon=1$ ). We choose $C_{1}$ large enough so that with the given constant $\epsilon$ (2.5) is met by both potentials. This will be used in Sect. 6 for simplifying the existence proof of the Møller transformations.

3 ) By a suitable translation of the origin, one could for $n \geq 2$ assume that $R_{\min }$ is, say, twice the maximal distance between the centres (but this assumption will not be used).

For $n \geq 2$ we set

$$
d_{\min }:=\min _{k \neq l} d^{k, l} \quad \text { and } \quad d_{\max }:=\max _{k \neq l} d^{k, l} \quad \text { with } \quad d^{k, l}:=\left|\vec{s}_{k}-\vec{s}_{l}\right| .
$$

In the case of a single atom $(n=1)$ we fix $d_{\min }$ by setting $d_{\min }:=2 R_{\min }$, say.

A threshold of the energy is

$$
V_{\max }:=\sup (\{V(\vec{q}) \mid \vec{q} \in \hat{M}, \nabla V(\vec{q})=\overrightarrow{0}\} \cup\{0\}) .
$$

One has $0 \leq V_{\max }<\infty$, and in many cases $V_{\max }=0$.

We will generally assume that there are no more than two nuclei on one line:

Definition 2.4. The configurations of singularities in

$$
\mathcal{N C}:=\left\{\left(\vec{s}_{1}, \ldots, \vec{s}_{n}\right) \in\left(\mathbb{R}_{\vec{q}}^{3}\right)^{n} \mid \forall i \neq j \neq k \neq i:\left(\vec{s}_{i}-\vec{s}_{j}\right) \times\left(\vec{s}_{j}-\vec{s}_{k}\right) \neq \overrightarrow{0}\right\}
$$

are called non-collinear (NC) configurations.

In three dimensions this is a weaker assumption than that the nuclei are in general position, since the latter also means that there are no more than three nuclei in one plane.

The space $\mathcal{N C}$ of configurations is a smooth connected manifold.

Most statements in this article are shown for values of $H$ above some threshold energy $E_{\text {th }}$. For given $W$ (in particular for the purely Coulombic case $W=0$ ) and charges $Z_{l}$ this is a function $E_{\mathrm{th}}: \mathcal{N C} \rightarrow \mathbb{R}$ of the positions $\left(\vec{s}_{1}, \ldots, \vec{s}_{n}\right)$ of singularities. $E_{\text {th }}$ diverges near the set $\left(\mathbb{R}_{\vec{q}}^{3}\right)^{n} \backslash \mathcal{N C}$ of partly collinear configurations. This is not an artefact of our methods of proof. Instead, we will see in Sect. 17 that several assertions proved in previous sections become wrong if one drops the NC condition. 
One parameter measuring the degree of non-collinearity of a configuration of $n \geq 3$ nuclei is the minimal angle

$$
\alpha_{\min }: \mathcal{N C} \rightarrow(0, \pi / 3] \quad \alpha_{\min }:=\min _{i \neq j \neq k \neq i} \alpha(i, j, k),
$$

where for $i \neq j \neq k$

$$
\alpha(i, j, k) \in[0, \pi), \quad \cos (\alpha(i, j, k)):=\left\langle\hat{s}^{j, i}, \hat{s}^{j, k}\right\rangle,
$$

with the directions

$$
\hat{s}^{i, j}:=\frac{\vec{s}_{j}-\vec{s}_{i}}{\left|\vec{s}_{j}-\vec{s}_{i}\right|}
$$

is the angle between $\vec{s}_{i}$ and $\vec{s}_{k}$, seen from $\vec{s}_{j}$. For $n=2$ we set $\alpha_{\min }:=\pi / 3$.

The Hamilton equations

$$
\dot{\vec{p}}=-\nabla V(\vec{q}) \quad, \quad \dot{\vec{q}}=\vec{p}
$$

lead to solutions $t \mapsto(\vec{p}(t), \vec{q}(t)) \equiv \Phi^{t}\left(x_{0}\right)$ of the initial value problem with initial values $x_{0}=\left(\vec{p}_{0}, \vec{q}_{0}\right)$ which exist uniquely up to eventual collisions with the $\vec{s}_{l}$. In Sect. 5 we extend $\Phi^{t}$ uniquely to a smooth complete flow.

The virial identity

$$
\frac{d}{d t}\langle\vec{q}(t), \vec{p}(t)\rangle=2(E-V(\vec{q}(t)))-\langle\vec{q}(t), \nabla V(\vec{q}(t))\rangle
$$

holds true for any trajectory $t \mapsto(\vec{p}(t), \vec{q}(t))$ with energy $E:=H\left(x_{0}\right)$ (whenever $\left.\vec{q}(t) \neq \vec{s}_{l}\right)$. Let us choose a function $R_{\mathrm{vir}}:(0, \infty) \rightarrow \mathbb{R}$ of the energy, called the virial radius, with

$$
\max \left(|V(\vec{q})|, \frac{\left|Z_{\infty}\right|}{|\vec{q}|}\right)<\frac{E}{2} \quad \text { and } \quad|\langle\vec{q}, \nabla V(\vec{q})\rangle|<E / 2 \quad\left(|\vec{q}| \geq R_{\mathrm{vir}}(E)\right) \text {. }
$$

As a consequence of part 2 of Definition 2.1 of Coulombic potentials, such a function exists. Without loss of generality (w.l.o.g.) we assume $R_{\text {vir }}$ to be continuous, nonincreasing and constant for energies $E>E_{\text {th }}$ above some threshold. Property (2.13) already implies that $R_{\text {vir }}(E)>2 Z_{\infty} / E$. Technically we choose $R_{\text {vir }}$ with

$$
R_{\mathrm{vir}}(E) \geq \max \left(2 R_{\min }, C_{2} / E\right) \quad \text { with } \quad C_{2}:=31(1+1 / \epsilon) R_{\min }^{1-\epsilon} C_{1}
$$

Then by (2.12) and (2.13)

$$
\frac{d}{d t}\langle\vec{q}(t), \vec{p}(t)\rangle>\frac{E}{2}>0 \quad \text { if }|\vec{q}(t)| \geq R_{\mathrm{vir}}(E) .
$$

We conclude that a configuration space trajectory $t \mapsto \vec{q}(t)$ of energy $E$ leaving the ball of radius $R_{\mathrm{vir}}(E)$ cannot reenter this ball in the future but must go to spatial infinity: 
Namely assume w.l.o.g. that $\langle\vec{q}(0), \vec{p}(0)\rangle \geq 0$. By (2.15)

$$
\frac{d^{2}}{d t^{2}} \vec{q}^{2}(t)=2 \frac{d}{d t}\langle\vec{q}(t), \vec{p}(t)\rangle>E \quad(t \geq 0)
$$

so that

$$
\vec{q}^{2}(t) \geq \vec{q}_{0}^{2}+\frac{1}{2} E t^{2} \quad(t \geq 0)
$$

We shall mainly deal with energies $E>E_{\text {th }}$ so that we may consider the $E$-independent interaction zone

$$
\mathcal{I Z}:=\left\{\vec{q} \in \mathbb{R}_{\vec{q}}^{3}|| \vec{q} \mid \leq R_{\mathrm{vir}}\left(E_{\mathrm{th}}\right)\right\} .
$$

Sometimes we will use outside $\mathcal{I Z}$ the shorthand

$$
V_{\mathrm{sr}}:=V-V_{\infty} \quad \text { with } \quad V_{\infty}(\vec{q}):=-\frac{Z_{\infty}}{|\vec{q}|}
$$

In addition to the condition (2.5) on $\nabla V_{\mathrm{sr}}$ this potential is of short range in the sense $V_{\text {sr }}(\vec{q})=\mathcal{O}\left(|\vec{q}|^{-1-\epsilon}\right)$, too, as follows from (2.4) and (2.5).

Due to collisions with the nuclei situated at $\vec{s}_{l}$, the flow on the phase space $T^{*} \hat{M}$ of the particle is incomplete. There are several ways to regularize the collision orbits which are all essentially equivalent. In Sect. 3 we now introduce the KustaanheimoStiefel (KS) regularization method, and use it in Sect. 4 for comparison estimates which control the deviation from the Keplerian motion near a singularity. Later, in Sect. 5, the flow is then regularized without a time change.

\section{The Kustaanheimo-Stiefel transformation}

In [KS] Kustaanheimo and Stiefel related the Kepler motion in three spatial dimensions to the motion of a resonant harmonic oscillator in four dimensions, thus linearizing the dynamics, see the book [StS] by Stiefel and Scheifele.

Although the authors had used spinor theory in order to derive their results, the emphasis of their article was more on the application to perturbation theory then on the geometry of the problem. In [Ku] Kummer presented this aspect of the $\mathrm{KS}$-transformation and related it to the approach [Mo] by Moser.

Our presentation will be based on the quaternion algebra over $\mathbb{R}$

$$
\mathbb{H}:=\left\{\left(\begin{array}{cc}
z_{1} & -z_{2} \\
\bar{z}_{2} & \bar{z}_{1}
\end{array}\right) \mid z_{1}, z_{2} \in \mathbb{C}\right\} \cong \mathbb{R}^{4}
$$

with matrix multiplication (see, e.g., $[\mathrm{KR}])$. We use the basis

$$
\begin{aligned}
\left(11, I_{1}, I_{2}, I_{3}\right) & :=\left(\left(\begin{array}{ll}
1 & 0 \\
0 & 1
\end{array}\right),\left(\begin{array}{cc}
0 & i \\
i & 0
\end{array}\right),\left(\begin{array}{cc}
0 & 1 \\
-1 & 0
\end{array}\right),\left(\begin{array}{cc}
i & 0 \\
0 & -i
\end{array}\right)\right) \\
& =\left(1, i \sigma_{1}, i \sigma_{2}, i \sigma_{3}\right)
\end{aligned}
$$

of $\mathbb{H}$, the $\sigma_{l}$ being the Pauli matrices. The direct sum decomposition

$$
\mathbb{H}=\mathbb{R} \cdot \mathbb{1} \oplus \operatorname{Im} \mathbb{H}
$$


with

$$
\begin{aligned}
\operatorname{Im} \mathbb{H} & :=\left\{Z \in \mathbb{H} \mid Z^{2}=\lambda \cdot 11 \text { with } \lambda \leq 0\right\} \\
& =\operatorname{Span}_{\mathbb{R}}\left(I_{1}, I_{2}, I_{3}\right)
\end{aligned}
$$

into real and imaginary space is orthogonal w.r.t. the inner product

$$
\mathbb{H} \times \mathbb{H} \rightarrow \mathbb{R} \quad, \quad\langle X, Y\rangle:=\frac{1}{2} \operatorname{tr}\left(X Y^{*}\right),
$$

$X \mapsto X^{*}:=\bar{X}^{t}$ being the conjugation. The norm $|X|:=\langle X, X\rangle^{\frac{1}{2}}$ is multiplicative:

$$
|X Y|=|X||Y| \quad(X, Y \in \mathbb{H}) .
$$

The vector product $\times: \operatorname{Im} \mathbb{H} \times \operatorname{Im} \mathbb{H} \rightarrow \operatorname{Im} \mathbb{H}$ is given by

$$
X \times Y:=\frac{1}{2}(X Y-Y X),
$$

and we have

$$
X Y=-\langle X, Y\rangle \mathbb{1}+X \times Y \quad(X, Y \in \operatorname{Im} \mathbb{H}) .
$$

We consider the Hopf map

$$
\pi_{0}: \mathbb{H} \rightarrow \operatorname{Im} \mathbb{H}, \quad \pi_{0}(Z):=Z^{*} I_{3} Z=i\left(\begin{array}{cc}
z_{1} \bar{z}_{1}-z_{2} \bar{z}_{2} & -2 \bar{z}_{1} z_{2} \\
-2 z_{1} \bar{z}_{2} & z_{2} \bar{z}_{2}-z_{1} \bar{z}_{1}
\end{array}\right)
$$

which is a surjection $\mathbb{R}^{4} \rightarrow \mathbb{R}^{3}$ whose preimages are the orbits of the isometric group action

$$
\alpha_{0}: S^{1} \rightarrow \operatorname{Aut}(\mathbb{H}), \quad \alpha_{0}(\varphi)(Z):=\exp \left(I_{3} \varphi\right) Z .
$$

This action is free on $\hat{\mathbb{H}}:=\mathbb{H} \backslash\{0\}$. We have the canonical symplectic one-forms

$$
\boldsymbol{\theta}:=\frac{1}{2} \operatorname{tr}\left(P^{*} d Q\right)=\Re\left(\bar{p}_{1} d q_{1}+\bar{p}_{2} d q_{2}\right) \quad\left((P, Q) \in T^{*} \mathbb{H}\right)
$$

and

$$
\theta:=\frac{1}{2} \operatorname{tr}\left(P^{*} d Q\right) \quad\left((P, Q) \in T^{*} \operatorname{Im} \mathbb{H}\right)
$$

on the cotangent bundles, and denote by $\hat{\boldsymbol{\theta}}:=\boldsymbol{\theta} \uparrow_{T * \hat{H}}$, resp. $\hat{\theta}:=\theta \uparrow_{T^{*} \operatorname{Im} \hat{H}}$ their restrictions.

The restricted Hopf map $\hat{\pi}_{0}:=\left.\pi_{0}\right|_{\hat{\mathbb{H}}}$ is then related to the $K S$-transformation

$$
\begin{aligned}
\hat{\pi} & : T^{*} \hat{\mathbb{H}} \rightarrow T^{*} \operatorname{Im} \hat{\mathbb{H}} \\
\hat{\pi}(P, Q) & =\left(\frac{-1}{4|Q|^{2}}\left(Q^{*} I_{3} P+P^{*} I_{3} Q\right), Q^{*} I_{3} Q\right)
\end{aligned}
$$

of the cotangent bundles.

We consider the quadric surface

$$
S:=\mathcal{I}^{-1}(0) \subset T^{*} \mathbb{H}
$$


for the bilinear form

$$
\mathcal{I}: T^{*} \mathbb{H} \rightarrow \mathbb{R}, \quad \mathcal{I}(P, Q):=\frac{1}{2} \operatorname{tr}\left(Q^{*} I_{3} P\right)=\Im\left(q_{1} \bar{p}_{1}+q_{2} \bar{p}_{2}\right)
$$

and its restriction $\hat{S}:=S \cap T^{*} \hat{\mathbb{H}}$. Then

$$
\hat{\boldsymbol{\theta}} \uparrow_{\hat{S}}=\hat{\pi}^{*} \hat{\theta} \uparrow_{\hat{S}},
$$

since on $\hat{S}$

$$
Q^{*} I_{3} P=P^{*} I_{3} Q \in \operatorname{Im} \mathbb{H} \text {. }
$$

$\mathcal{I}, \boldsymbol{\theta}$ and $\hat{\pi}$ are all invariant w.r.t. the group action

$$
\alpha: S^{1} \rightarrow \operatorname{Aut}\left(T^{*} \mathbb{H}\right), \quad \alpha(\varphi)(P, Q):=\left(\exp \left(I_{3} \varphi\right) P, \exp \left(I_{3} \varphi\right) Q\right),
$$

and $\mathcal{I}$ is a Hamiltonian function generating that time- $\varphi$-flow.

Lemma 3.1. Restricted to the quadric surface $\hat{S} \subset T^{*} \mathbb{H}$, the lift of a Hamiltonian function $\hat{H}(\vec{p}, \vec{q})=\frac{1}{2}|\vec{p}|^{2}-Z /|\vec{q}|+W(\vec{q})$ equals

$$
\hat{H} \circ \hat{\pi}(P, Q)=|Q|^{-2}\left(\frac{1}{8}|P|^{2}-Z\right)+W\left(Q^{*} I_{3} Q\right) \quad((P, Q) \in \hat{S}) .
$$

For the angular momentum $\vec{L}(\vec{p}, \vec{q})=\vec{q} \times \vec{p}$

$$
\vec{L} \circ \hat{\pi}(P, Q)=\frac{1}{4}\left(Q^{*} P-P^{*} Q\right) \quad((P, Q) \in \hat{S}),
$$

whereas the Runge-Lenz vector $\vec{F}(\vec{p}, \vec{q})=\vec{p} \times \vec{L}-Z \frac{\vec{q}}{|\vec{q}|}$ transforms into

$$
\begin{array}{r}
\vec{F} \circ \hat{\pi}(P, Q)=-\frac{1}{8} P^{*} I_{3} P+\left(\hat{H} \circ \hat{\pi}(P, Q)-W\left(Q^{*} I_{3} Q\right)\right) \cdot Q^{*} I_{3} Q \\
((P, Q) \in \hat{S}) .
\end{array}
$$

Proof. (see also $[\mathrm{Ku}]$ ).

By (3.4) the restricted KS-transformation $\pi{ }^{\mid} \hat{s}_{S}$ maps $|Q|^{2}=\left|Q^{*} I_{3} Q\right|$ onto $|q|$ and $|2 Q|^{-2} \cdot|P|^{2}=\left|\left(2|Q|^{2}\right)^{-1} Q^{*} I_{3} P\right|^{2}$ onto $|p|^{2}$, implying (3.6).

Since $q, p \in \operatorname{Im} \mathbb{H}$,

$$
\vec{L}=q \times p=\frac{1}{2}(q p-p q) .
$$

The KS-transformation $\hat{\pi}$ gives, using (3.4)

$$
\begin{aligned}
\vec{L} \circ \hat{\pi}(P, Q) & =\frac{\left(Q^{*} I_{3} P+P^{*} I_{3} Q\right) Q^{*} I_{3} Q-Q^{*} I_{3} Q\left(Q^{*} I_{3} P+P^{*} I_{3} Q\right)}{8|Q|^{2}} \\
& =\frac{P^{*} I_{3} Q Q^{*} I_{3} Q-Q^{*} I_{3} Q Q^{*} I_{3} P}{4|Q|^{2}}=\frac{1}{4}\left(Q^{*} P-P^{*} Q\right),
\end{aligned}
$$

This shows (3.7). To prove (3.8), we use the identity

$$
p \times(q \times p)=\frac{1}{2}(p q p-q p p)=\frac{1}{2}\left(p q p+q|p|^{2}\right) \quad(p, q \in \mathrm{Im} \mathbb{H})
$$


which implies that

$$
\vec{F}(\vec{p}, \vec{q})=\frac{1}{2} p q p+q \cdot(H(p, q)-W(q)) .
$$

The term $p q p$ lifts to

$$
\left(2|Q|^{2}\right)^{-2}\left(P^{*} I_{3} Q\right)\left(Q^{*} I_{3} Q\right)\left(Q^{*} I_{3} P\right)=-\frac{1}{4} P^{*} I_{3} P,
$$

finishing the proof.

\section{Application of the KS-transformation}

In a configuration space ball

$$
B_{l}(r):=\left\{\vec{q} \in \mathbb{R}_{\vec{q}}^{3}|| \vec{q}-\vec{s}_{l} \mid \leq r\right\}
$$

of radius $r:=c_{q}<\frac{1}{2} d_{\text {min }}$ around $\vec{q}=\vec{s}_{l}$ we want to regularize the motion generated by the restricted Hamiltonian function

$$
\begin{gathered}
\hat{H}(\vec{p}, \vec{q})=\frac{1}{2}|\vec{p}|^{2}-\frac{Z_{l}}{\left|\vec{q}-\vec{s}_{l}\right|}+W_{l}(\vec{q}), \quad\left((\vec{p}, \vec{q}) \in T^{*}\left(B_{l}\left(c_{q}\right) \backslash\{\overrightarrow{0}\}\right)\right) \\
W_{l}(\vec{q}):=\sum_{i \neq l} \frac{-Z_{i}}{\left|\vec{q}-\vec{s}_{i}\right|}+W(\vec{q})
\end{gathered}
$$

being a smooth function on $B_{l}\left(c_{q}\right) \subset \mathbb{R}^{3} \cong \operatorname{Im} \mathbb{H}$. The radius $c_{q} \in\left(0, \frac{1}{2} d_{\text {min }}\right)$ is chosen so that

$$
\left|W_{l}(\vec{q})\right| \leq \frac{1}{2} \frac{\left|Z_{l}\right|}{\left|\vec{q}-\vec{s}_{l}\right|} \quad\left(l=1, \ldots, n,\left|\vec{q}-\vec{s}_{l}\right| \leq c_{q}\right) .
$$

For simplicity of notation we assume $\vec{s}_{l}=\overrightarrow{0}$. For regular values $E$ of the energy $\hat{H}$ the orbits on $\hat{H}^{-1}(E)$ coincide with the ones of the zero surface of the Hamiltonian function

$$
|q| \cdot(\hat{H}(p, q)-E)
$$

since they are already determined by the form of the submanifold $\hat{H}^{-1}(E)$.

By (3.6) the lift of (4.3) with the KS-transformation equals

$$
\mathbf{H}_{E}(P, Q):=\frac{1}{8}\langle P, P\rangle+\langle Q, Q\rangle\left(-E+W_{l}\left(Q^{*} I_{3} Q\right)\right)-Z_{l} .
$$

The orbits of its Hamiltonian vector field on $\hat{S} \cap\left(\mathbf{H}_{E}\right)^{-1}(0)$ project to the ones of $\hat{H}$ on $\hat{H}^{-1}(E)$, since the symplectic one-form $\hat{\theta}$ on the phase space region $T^{*}\left(B_{l}\left(c_{q}\right) \backslash\{\overrightarrow{0}\}\right)$ lifts according to (3.3).

For $W_{l} \equiv 0$ the Hamiltonian function $\mathbf{H}_{E}$ is the one of a four-dimensional harmonic oscillator (with negative potential for $E>0$ ). In the general case the additional potential $Q \mapsto W_{l}\left(Q^{*} I_{3} Q\right)$ will be a small perturbation if $E$ is large, at least for $|Q| \leq \sqrt{c_{q}}$. 
From now on we assume initial conditions to lie in $S \subset T^{*} \mathbb{H}$.

Instead of considering the Hamilton equations

$$
\left\{\begin{array}{l}
\frac{d}{d s} P=2 E Q+2 R(Q) \\
\frac{d}{d s} Q=\frac{1}{4} P
\end{array}\right.
$$

of (4.4) with perturbation

$$
R(Q):=-Q W_{l}\left(Q^{*} I_{3} Q\right)-\frac{1}{2}|Q|^{2} \nabla W_{l}\left(Q^{*} I_{3} Q\right),
$$

we want the parameter $E$ to appear only in the perturbative term. Thus we set

$$
X:=\left(\begin{array}{l}
\tilde{P} \\
Q
\end{array}\right) \quad \text { with } \quad \tilde{P}:=P / \sqrt{8 E}
$$

and use the time variable $\tau:=\sqrt{E / 2} \cdot s$. Then

$$
\frac{d}{d \tau} X=\left(\begin{array}{ll}
0 & 11 \\
1 & 0
\end{array}\right) X+\tilde{R}(X) / E \quad, \quad X(0)=X_{0} \equiv\left(\begin{array}{l}
\tilde{P}_{0} \\
Q_{0}
\end{array}\right)
$$

with $\tilde{R}(X):=\left(\begin{array}{c}R(Q) \\ 0\end{array}\right)$.

The initial conditions $X_{0}:=\left(\begin{array}{c}P_{0} / \sqrt{8 E} \\ Q_{0}\end{array}\right)$ meet the energy constraint

$$
\left|\tilde{P}_{0}\right|^{2}-\left|Q_{0}\right|^{2}\left(1-W_{l}\left(Q_{0}^{*} I_{3} Q_{0}\right) / E\right)=\frac{Z_{l}}{E} .
$$

So with

$$
c_{Q}:=\sqrt{c_{q}}
$$

and

$$
\mathcal{E}(\tilde{P}, Q):=\frac{Z_{l}-|Q|^{2} W_{l}\left(Q^{*} I_{3} Q\right)}{|\tilde{P}|^{2}-|Q|^{2}}
$$

the region

$$
\mathcal{D}_{l}:=\left\{(\tilde{P}, Q) \in \mathbb{R}^{4} \times \mathbb{R}^{4}|| Q \mid \leq c_{Q}, \mathcal{E}(\tilde{P}, Q)>E_{\mathrm{th}}\right\}
$$

of phase space points meeting (4.7) for some $E>E_{\text {th }}$ is bounded.

It is natural to compare the solution $X(\tau)$ of (4.6) with the solution

$$
Y(\tau)=\left(\begin{array}{ll}
\cosh (\tau) \mathbb{1} & \sinh (\tau) \mathbb{1} \\
\sinh (\tau) \mathbb{1} & \cosh (\tau) \mathbb{1 1}
\end{array}\right) X_{0}
$$

of the linear initial value problem

$$
\frac{d}{d \tau} Y=\left(\begin{array}{ll}
0 & 11 \\
11 & 0
\end{array}\right) Y \quad, \quad Y(0)=X_{0} .
$$


We denote this linear flow (corresponding to $W_{l} \equiv 0$ ), restricted to the invariant phase space domain

$$
\breve{\mathcal{D}}_{L, l}:=\left\{(\tilde{P}, Q) \in \mathbb{R}^{4} \times \mathbb{R}^{4}|| \tilde{P}|\neq| Q \mid, \mathcal{E}_{L}(\tilde{P}, Q)>\frac{1}{2} E_{\mathrm{th}}\right\}
$$

with

$$
\mathcal{E}_{L}(\tilde{P}, Q):=Z_{l} /\left(|\tilde{P}|^{2}-|Q|^{2}\right)
$$

by

$$
\boldsymbol{\Phi}_{L, l}: \mathbb{R} \times \breve{\mathcal{D}}_{L, l} \rightarrow \breve{\mathcal{D}}_{L, l}
$$

and set

$$
\mathcal{D}_{L, l}:=\left\{(\tilde{P}, Q) \in \breve{\mathcal{D}}_{L, l}|| Q \mid \leq c_{Q}\right\} .
$$

Our condition (4.2) on the radius $c_{q}$ implies that

$$
\mathcal{D}_{L, l} \supset \mathcal{D}_{l}
$$

Let

$$
\mathbf{T}_{l}^{ \pm}: \mathcal{D}_{l} \rightarrow \mathbb{R} \cup\{ \pm \infty\}, \quad \mathbf{T}_{l}^{ \pm}\left(X_{0}\right):= \pm \inf \left\{t>0|| Q\left( \pm t, X_{0}\right) \mid \geq c_{Q}\right\} .
$$

be the exit times from $\mathcal{D}_{l}$,

$$
\boldsymbol{\Phi}_{l}: \mathbf{U}_{l} \rightarrow \mathcal{D}_{l}, \quad \mathbf{U}_{l}:=\left\{\left(t, X_{0}\right) \in \mathbb{R} \times \mathcal{D}_{l} \mid t \in\left[\mathbf{T}_{l}^{-}\left(X_{0}\right), \mathbf{T}_{l}^{+}\left(X_{0}\right)\right]\right\}
$$

the maximally extended KS flow on $\mathcal{D}_{l}$, and

$$
\boldsymbol{\Psi}_{l}^{ \pm}: \mathcal{D}_{l} \rightarrow \partial \mathcal{D}_{l} \quad, \quad X \mapsto \boldsymbol{\Phi}_{l}\left(\mathbf{T}_{l}^{ \pm}(X), X\right)
$$

the map to the exit points.

Basically we are interested in the Poincaré map

$$
\boldsymbol{\Psi}_{l}: \partial \mathcal{D}_{l} \rightarrow \partial \mathcal{D}_{l}, \quad X \equiv(\tilde{P}, Q) \mapsto\left\{\begin{array}{l}
\boldsymbol{\Psi}_{l}^{+}(X),\langle\tilde{P}, Q\rangle \leq 0 \\
\boldsymbol{\Psi}_{l}^{-}(X),\langle\tilde{P}, Q\rangle>0
\end{array}\right.
$$

that permutes incoming and outgoing data, but up to now we do not even know whether this is defined everywhere.

Therefore we compare with the linear flow (4.12) and thus introduce in analogy its exit times $\mathbf{T}_{L, l}^{ \pm}: \mathcal{D}_{L, l} \rightarrow \mathbb{R}$. They are finite and smooth, and we set $\boldsymbol{\Psi}_{L, l}^{ \pm}(X):=$ $\boldsymbol{\Phi}_{L, l}\left(\mathbf{T}_{L, l}^{ \pm}(X), X\right)$.

The analog

$$
\boldsymbol{\Psi}_{L, l}: \partial \mathcal{D}_{L, l} \rightarrow \partial \mathcal{D}_{L, l}, \quad X \equiv(\tilde{P}, Q) \mapsto\left\{\begin{array}{l}
\Psi_{L, l}^{+}(X),\langle\tilde{P}, Q\rangle \leq 0 \\
\Psi_{L, l}^{-}(X),\langle\tilde{P}, Q\rangle>0
\end{array}\right.
$$


of (4.13) for the linear flow is a involutive diffeomorphism:

$$
\Psi_{L, l}\left(\begin{array}{l}
\tilde{P} \\
Q
\end{array}\right)=\frac{1}{\sqrt{1-u^{2}}}\left(\begin{array}{l}
\tilde{P}-u Q \\
Q-u \tilde{P}
\end{array}\right) \text { with } \quad u:=\frac{2\langle\tilde{P}, Q\rangle}{|\tilde{P}|^{2}+|Q|^{2}},
$$

since $|u|<1$ on $\mathcal{D}_{L, l}$.

Ideally, one would like to prove that (4.13) is approximated by the map (4.14) so that the $C^{1}$-norm of $\boldsymbol{\Psi}_{l} \circ\left(\boldsymbol{\Psi}_{L, l}\right)^{-1}-\operatorname{Id}$ is of order $\mathcal{O}(1 / \mathcal{E})$. This would be true if the trajectories of $\boldsymbol{\Phi}_{l}$, resp. $\boldsymbol{\Phi}_{L, l}$ would spend a uniformly bounded time inside $\mathcal{D}_{l}$, resp. $\mathcal{D}_{L, l}$. But this is not the case, and in order to keep the error terms small we will compare the two flows for initial conditions on the pericentric hypersurface

$$
\mathcal{H}_{l}:=\left\{(\tilde{P}, Q) \in \mathcal{D}_{l} \mid\langle\tilde{P}, Q\rangle=0\right\} \subset \mathcal{H}_{L, l}:=\left\{(\tilde{P}, Q) \in \breve{\mathcal{D}}_{L, l} \mid\langle\tilde{P}, Q\rangle=0\right\}
$$

Note that by transversality of the linear flow to that hypersurface the pericentric time

$$
\mathbf{T}_{L, l}^{0}: \breve{\mathcal{D}}_{L, l} \rightarrow \mathbb{R} \quad \text { with } \quad \boldsymbol{\Psi}_{L, l}^{0}(X):=\boldsymbol{\Phi}_{L, l}\left(\mathbf{T}_{L, l}^{0}(X), X\right) \in \mathcal{H}_{L, l}
$$

is uniquely defined and smooth.

Proposition 4.1. For $E_{\mathrm{th}}$ large there is a unique pericentric time $\mathbf{T}_{l}^{0}: \mathcal{D}_{l} \rightarrow \mathbb{R}$ with

$$
\boldsymbol{\Psi}_{l}^{0}(X):=\boldsymbol{\Phi}_{l}\left(\mathbf{T}_{l}^{0}(X), X\right) \in \mathcal{H}_{l}, \quad\left(X \in \mathcal{D}_{l}\right) .
$$

The functions $\mathbf{T}_{l}^{-} \leq \mathbf{T}_{l}^{0} \leq \mathbf{T}_{l}^{+}: \mathcal{D}_{l} \rightarrow \mathbb{R}$ are smooth, and

$$
\left|\mathbf{T}_{l}^{ \pm}\left(X_{0}\right)-\mathbf{T}_{L, l}^{ \pm}\left(X_{0}\right)\right|=\mathcal{O}\left(1 / \mathcal{E}\left(X_{0}\right)\right) \quad\left(X_{0} \in \mathcal{H}_{l}\right) .
$$

The exit times $\mathbf{T}_{L, l}^{ \pm}$of the linear flow are estimated by

$$
\exp \left( \pm 2 \mathbf{T}_{L, l}^{ \pm}\left(X_{0}\right)\right)=\frac{4 c_{q}}{\left|Z_{l}\right| e\left(X_{0}\right)} \mathcal{E}\left(X_{0}\right)+\mathcal{O}\left(\mathcal{E}^{0}\left(X_{0}\right)\right) \quad\left(X_{0} \in \mathcal{H}_{l}\right)
$$

$e\left(X_{0}\right):=\sqrt{1+\frac{2 E\left|\vec{L}\left(X_{0}\right)\right|^{2}}{Z_{l}^{2}}}$ being the eccentricity of the corresponding Kepler hyperbola. The diffeomorphism

$$
\boldsymbol{\Xi}_{l}: \partial \mathcal{D}_{l} \rightarrow \partial \mathcal{D}_{L, l}, \quad X \equiv(\tilde{P}, Q) \mapsto\left\{\begin{array}{l}
\boldsymbol{\Psi}_{L, l}^{-} \circ \boldsymbol{\Psi}_{l}^{0}(X),\langle\tilde{P}, Q\rangle \leq 0 \\
\boldsymbol{\Psi}_{L, l}^{+} \circ \boldsymbol{\Psi}_{l}^{0}(X),\langle\tilde{P}, Q\rangle>0
\end{array}\right.
$$

onto its image which conjugates the maps $\left(\boldsymbol{\Psi}_{l}=\left(\boldsymbol{\Xi}_{l}\right)^{-1} \circ \boldsymbol{\Psi}_{L, l} \circ \boldsymbol{\Xi}_{l}\right)$ is $C^{0}$-near to the identity in the sense that

$$
\left|\boldsymbol{\Xi}_{l}(X)-X\right|=\mathcal{O}(1 / \mathcal{E}(X)), \quad\left(X \in \partial \mathcal{D}_{l}\right),
$$

and the solution $\boldsymbol{\Psi}_{L, l}$ of the linear problem is $C^{1}$-near to $\boldsymbol{\Psi}_{l}$ in the sense

$$
\begin{aligned}
\left|\boldsymbol{\Psi}_{l}(X)-\boldsymbol{\Psi}_{L, l} \circ \boldsymbol{\Xi}_{l}(X)\right| & =\mathcal{O}(1 / \mathcal{E}(X)) \\
\left\|D \boldsymbol{\Psi}_{l}(X)-D \boldsymbol{\Psi}_{L, l} \circ \boldsymbol{\Xi}_{l}(X)\right\| & =\mathcal{O}(1 / e(X)) .
\end{aligned}
$$


Remarks 4.2. 1) The energy-independent estimate (4.21) may seem to be poor but is in fact optimal in its energy dependence since, relative to the optimal estimates

$$
\left\|D \Psi_{l}(X)\right\|=\mathcal{O}(\mathcal{E}(X) / e(X))=\left\|D \Psi_{L, l}(X)\right\|,
$$

it is of order $\mathcal{O}(1 / E)$ (The r.h.s. of (4.22) is obtained by inserting the time bound (4.18) into the linearization of (4.9)).

2) For pericentric initial data $X_{0} \in \mathcal{H}_{l}$ the total time spent inside the ball equals $\mathbf{T}_{L, l}^{+}\left(X_{0}\right)-\mathbf{T}_{L, l}^{-}\left(X_{0}\right) \equiv \pm 2 \mathbf{T}_{L, l}^{ \pm}\left(X_{0}\right)$. Estimate (4.18) for that time is presented in a form needed to evaluate the term $D \boldsymbol{\Psi}_{L, l} \circ \boldsymbol{\Xi}_{l}(X)$ in (4.21).

In polar coordinates $(r, \varphi)$ the Kepler hyperbola has the parametric form (see e.g. [Th], Chapter 4.2)

$$
r(\varphi)=\frac{\vec{L}^{2}}{|Z| e \cos \left(\varphi-\varphi_{0}\right)+Z} .
$$

The denominator has the zeros $\varphi^{ \pm}$, and $\Delta \varphi:=\varphi^{+}-\varphi^{-}$is the angle under which the hyperbola is seen from the origin. Thus

$$
\cos \left(\frac{1}{2} \Delta \varphi\right)=-\frac{\operatorname{sign}(Z)}{e},
$$

so that $\Delta \varphi \in(\pi, 2 \pi]$ for $Z>0$ and $\Delta \varphi \in[0, \pi)$ for $Z<0$.

On the other hand, the total change in direction $\Delta \psi$ of the velocity vector equals

$$
\Delta \psi=\operatorname{sign}(Z) \cdot(\Delta \varphi-\pi)=2 \arcsin \left(1 / e\left(X_{0}\right)\right) \in(0, \pi] .
$$

Thus (4.18) can be rewritten as

$$
\exp \left( \pm 2 \mathbf{T}_{L, l}^{ \pm}\left(X_{0}\right)\right)=\frac{4 c_{q} \sin \left(\frac{1}{2} \Delta \psi\right)}{\left|Z_{l}\right|} \mathcal{E}\left(X_{0}\right)+\mathcal{O}\left(\mathcal{E}^{0}\left(X_{0}\right)\right) \quad\left(X_{0} \in \mathcal{H}_{l}\right)
$$

This equation will be useful for the study of orbit instability, since exp $(t)$ equals the expansion of the unstable manifold of (4.9) after time $t$.

Proof. We set $(\tilde{P}(t), Q(t)):=\Phi_{l}\left(t, X_{0}\right)$ for $X_{0}=\left(\tilde{P}_{0}, Q_{0}\right) \in \mathcal{D}_{l}$. For $E_{\text {th }}$ large the squared distance $t \mapsto|Q(t)|^{2}$ is a strictly convex function of time, since

$$
\begin{aligned}
\frac{1}{2} \frac{d^{2}}{d t^{2}}|Q(t)|^{2} & =\frac{d}{d t}\langle\tilde{P}(t), Q(t)\rangle \\
& =|\tilde{P}(t)|^{2}+|Q(t)|^{2}-\langle R(Q(t)), Q(t)\rangle / \mathcal{E}\left(X_{0}\right) \\
& \geq|\tilde{P}(t)|^{2}+|Q(t)|^{2} \cdot\left(1-\mathcal{L}_{1} c_{q} / \mathcal{E}\left(X_{0}\right)\right) \\
& \geq \frac{1}{2}\left(|\tilde{P}(t)|^{2}+|Q(t)|^{2}\right)>0
\end{aligned}
$$

with Lipschitz constant 


$$
\mathcal{L}_{1}:=\sup \left\{\frac{\left|R\left(Q_{1}\right)-R\left(Q_{2}\right)\right|}{\left|Q_{1}-Q_{2}\right|}|| Q_{1}|,| Q_{2} \mid \leq c_{Q}, Q_{1} \neq Q_{2}\right\},
$$

(one notes from inspection of (4.5) that $R(0)=0$ ).

We can bound (4.24) more precisely from below by using the inequality

$$
|X|^{2} \equiv|\tilde{P}|^{2}+|Q|^{2} \geq\left.\frac{1}{2}|| \tilde{P}\right|^{2}-|Q|^{2}\left(1-W_{l}\left(Q^{*} I_{3} Q\right) / \mathcal{E}(X)\right) \mid=\frac{\left|Z_{l}\right|}{2 \mathcal{E}(X)}
$$

which follows from (4.7) and is valid for $E_{\text {th }}>\max _{\vec{q} \in B_{l}\left(c_{q}\right)}\left|W_{l}(\vec{q})\right|$.

Thus $\mathbf{T}_{l}^{ \pm}$and $\mathbf{T}_{l}^{0}$ are uniquely defined finite functions. By transversality of $\mathcal{H}_{l}$ w.r.t. the flow the pericentric time $\mathbf{T}_{l}^{0}$ is smooth. The hypersurface $\partial \mathcal{D}_{l}$ is transversal to the flow, too, except at $\partial \mathcal{D}_{l} \cap \mathcal{H}_{l}$. Thus it is only there that we have to control smoothness of the exit times $\mathbf{T}_{l}^{ \pm}$.

The maps

$$
\mathcal{D}_{l} \rightarrow \mathbb{R} \times \mathcal{H}_{l}, \quad X \mapsto\left(\mathbf{T}_{l}^{0}(X), \Psi_{l}^{0}(X)\right)
$$

and

$$
\mathbb{R} \times \mathcal{H}_{l} \rightarrow \mathbb{R}^{4} \times \mathbb{R}^{4}, \quad(t, X) \mapsto \boldsymbol{\Phi}_{L, l}(-t, X)
$$

are diffeomorphisms onto their images, since $\mathcal{D}_{L, l}$ does not contain the (single) stationary point 0 of the linear flow $\boldsymbol{\Phi}_{L, l}$. Hence the composition

$$
\rho_{l}: \mathcal{D}_{l} \rightarrow \breve{\mathcal{D}}_{L, l}, \quad \rho_{l}(X):=\boldsymbol{\Phi}_{L, l}\left(-\mathbf{T}_{l}^{0}(X), \mathbf{\Psi}_{l}^{0}(X)\right)
$$

of these diffeos is a diffeomorphism onto its image.

Thus in order to compare the flows $\boldsymbol{\Phi}_{l}$ and $\boldsymbol{\Phi}_{L, l}$, it suffices to compare the trajectories

$$
X(t):=(\tilde{P}(t), Q(t)):=\boldsymbol{\Phi}_{l}\left(t, X_{0}\right) \text { and } Y(t):=\left(\tilde{P}_{L}(t), Q_{L}(t)\right):=\boldsymbol{\Phi}_{L, l}\left(t, X_{0}\right)
$$

for pericentric initial conditions $X_{0}=\left(\tilde{P}_{0}, Q_{0}\right) \in \mathcal{H}_{l}$.

We partition $\mathcal{H}_{l}$ into the regions $\mathcal{H}_{l}^{<}:=\left\{(\tilde{P}, Q) \in \mathcal{H}_{l}|| Q \mid \leq \frac{1}{2} c_{Q}\right\}$ and

$$
\mathcal{H}_{l}^{>}:=\left\{(\tilde{P}, Q) \in \mathcal{H}_{l}\left|\frac{1}{2} c_{Q} \leq\right| Q \mid \leq c_{Q}\right\} .
$$

The flow through $\mathcal{H}_{l}<$ is uniformly transversal to $\partial \mathcal{D}_{L, l}$, whereas the exit times are uniformly bounded on $\mathcal{H}_{l}>$.

1) On $\mathcal{H}_{l}^{>}$the second equation in (4.24) together with (4.7) yields for

$$
g_{1}(t):=|Q(t)|^{2} \quad \frac{1}{2} \frac{d^{2}}{d t^{2}} g_{1}(t)-2 g_{1}(t)=h_{1}(t) / \mathcal{E}\left(X_{0}\right)
$$

with

$$
\left.h_{1}(t):=Z_{l}-\langle R(Q(t)), Q(t)\rangle\right)-|Q(t)|^{2} W_{l}\left(Q^{*}(t) I_{3} Q(t)\right) \quad\left(|t| \leq \mathbf{T}_{l}^{+}\left(X_{0}\right)\right),
$$


$g_{1}(0)=\left|Q_{0}\right|^{2}$ and $g_{1}^{\prime}(0)=0$ so that

$$
g_{1}(t)=\left|Q_{0}\right|^{2}+\int_{0}^{t} \int_{0}^{s}\left(4 g_{1}(u)+2 h_{1}(u) / \mathcal{E}\left(X_{0}\right)\right) d u d s .
$$

As $\left.C_{3}:=\sup _{|Q| \leq c_{Q}} \mid Z_{l}-\langle R(Q), Q\rangle\right)-|Q|^{2} W_{l}\left(Q^{*} I_{3} Q\right) \mid<\infty$, for $E_{\text {th }}$ large and times $t$ between 0 and $\mathbf{T}_{l}^{ \pm}\left(X_{0}\right)$ the integrand is bounded below by $3\left|Q_{0}\right|^{2}$ and above by $5 c_{Q}^{2}$ so that

$$
\frac{3}{2}\left|Q_{0}\right|^{2} t^{2} \leq g_{1}(t) \leq \frac{5}{2} c_{Q}^{2} t^{2}
$$

As $g_{1}\left(\mathbf{T}_{l}^{ \pm}\left(X_{0}\right)\right)=c_{Q}^{2}$,

$\frac{1}{2} \sqrt{1-\left|Q_{0}\right|^{2} / c_{Q}^{2}} \leq \pm \mathbf{T}_{l}^{ \pm}\left(X_{0}\right) \leq 2 \sqrt{1-\left|Q_{0}\right|^{2} / c_{Q}^{2}} \quad\left(X_{0} \equiv\left(\tilde{P}_{0}, Q_{0}\right) \in \mathcal{H}_{l}^{>}\right)$,

and the same estimate holds for $\mathbf{T}_{L, l}^{ \pm}$. Est. (4.29) implies in particular the uniform bound $\left|\mathbf{T}_{l}^{ \pm}\right|,\left|\mathbf{T}_{L, l}^{ \pm}\right| \leq 2$ on $\mathcal{H}_{l}^{>}$.

The difference between these times is much smaller:

$$
\left|\mathbf{T}_{l}^{ \pm}\left(X_{0}\right)-\mathbf{T}_{L, l}^{ \pm}\left(X_{0}\right)\right|=\mathcal{O}\left(\sqrt{1-\left|Q_{0}\right|^{2} / c_{Q}^{2}} / \mathcal{E}\left(X_{0}\right)\right) \quad\left(X_{0} \in \mathcal{H}_{l}^{>}\right) .
$$

Namely setting $g_{2}(t):=|Q(t)|^{2}-\left|Q_{L}(t)\right|^{2}$, so that $g_{2}(0)=g_{2}^{\prime}(0)=0$ and, by (4.28),

$$
\frac{1}{2} \frac{d^{2}}{d t^{2}} g_{2}(t)-2 g_{2}(t)=\frac{h_{2}(t)}{\mathcal{E}\left(X_{0}\right)} \quad \text { with } \quad h_{2}(t):=h_{1}(t)-Z_{l},
$$

we get

$$
g_{2}(t)=\int_{0}^{t} \int_{0}^{s}\left(4 g_{2}(u)+2 h_{2}(u) / \mathcal{E}\left(X_{0}\right)\right) d u d s
$$

or

$$
\left|g_{2}(t)\right| \leq \frac{C_{4}}{\mathcal{E}\left(X_{0}\right)} \sinh ^{2}(t)
$$

for $C_{4}:=C_{3}+Z_{\max }$.

$$
\begin{aligned}
& \text { As } c_{Q}^{2}=\left|Q_{L}\left(\mathbf{T}_{L, l}^{ \pm}\left(X_{0}\right)\right)\right|^{2}=\sinh ^{2}\left(\mathbf{T}_{L, l}^{ \pm}\left(X_{0}\right)\right)\left|Q_{0}\right|^{2} \text { and } \\
& g_{2}\left(\mathbf{T}_{l}^{ \pm}\left(X_{0}\right)\right)=c_{Q}^{2}-\left|Q_{L}\left(\mathbf{T}_{l}^{ \pm}\left(X_{0}\right)\right)\right|^{2}=c_{Q}^{2}-\sinh ^{2}\left(\mathbf{T}_{l}^{ \pm}\left(X_{0}\right)\right)\left|Q_{0}\right|^{2}, \\
& \sinh \left(\mathbf{T}_{l}^{ \pm}\left(X_{0}\right)\right) \cdot \sqrt{1-\frac{C_{4}}{\left|Q_{0}\right|^{2} \mathcal{E}\left(X_{0}\right)}} \leq \sinh \left(\mathbf{T}_{L, l}^{ \pm}\left(X_{0}\right)\right) \\
& \leq \sinh \left(\mathbf{T}_{l}^{ \pm}\left(X_{0}\right)\right) \cdot \sqrt{1+\frac{C_{4}}{\left|Q_{0}\right|^{2} \mathcal{E}\left(X_{0}\right)}} .
\end{aligned}
$$

In view of (4.29) this gives (4.30). 
In turn (4.30) implies

$$
\begin{aligned}
& \left|\boldsymbol{\Phi}_{L, l}\left(\mathbf{T}_{L, l}^{ \pm}\left(X_{0}\right), X_{0}\right)-\boldsymbol{\Phi}_{L, l}\left(\mathbf{T}_{l}^{ \pm}\left(X_{0}\right), X_{0}\right)\right|+ \\
& \left\|D \boldsymbol{\Phi}_{L, l}\left(\mathbf{T}_{L, l}^{ \pm}\left(X_{0}\right), X_{0}\right)-D \boldsymbol{\Phi}_{L, l}\left(\mathbf{T}_{l}^{ \pm}\left(X_{0}\right), X_{0}\right)\right\| \\
& =\mathcal{O}\left(\sqrt{1-\left|Q_{0}\right|^{2} / c_{Q}^{2}} / \mathcal{E}\left(X_{0}\right)\right) \text {. }
\end{aligned}
$$

A comparison between the initial value problems (4.6) and (4.10) using a Gronwall estimate on the uniformly bounded time interval $|t| \leq 2$ yields

$$
\begin{array}{r}
\left|\boldsymbol{\Phi}_{l}^{t}\left(X_{0}\right)-\boldsymbol{\Phi}_{L, l}^{t}\left(X_{0}\right)\right|+\left\|D \boldsymbol{\Phi}_{l}^{t}\left(X_{0}\right)-D \boldsymbol{\Phi}_{L, l}^{t}\left(X_{0}\right)\right\| \leq \frac{C|t|}{\mathcal{E}\left(X_{0}\right)} \\
\left(X_{0} \in \mathcal{H}_{l}^{>}, t \in\left[\mathbf{T}_{l}^{-}\left(X_{0}\right), \mathbf{T}_{l}^{+}\left(X_{0}\right)\right]\right) .
\end{array}
$$

Setting $t:=\mathbf{T}_{l}^{ \pm}\left(X_{0}\right)$ in (4.32), the triangle inequality and (4.31) leads to

$$
\begin{gathered}
\left|\boldsymbol{\Psi}_{l}^{ \pm}\left(X_{0}\right)-\boldsymbol{\Psi}_{L, l}^{ \pm}\left(X_{0}\right)\right|+\left\|D \boldsymbol{\Psi}_{l}^{ \pm}\left(X_{0}\right)-D \boldsymbol{\Psi}_{L, l}^{ \pm}\left(X_{0}\right)\right\| \equiv \\
\left|\boldsymbol{\Phi}_{l}\left(\mathbf{T}_{l}^{ \pm}\left(X_{0}\right), X_{0}\right)-\boldsymbol{\Phi}_{L, l}\left(\mathbf{T}_{L, l}^{ \pm}\left(X_{0}\right), X_{0}\right)\right|+ \\
\left\|D \boldsymbol{\Phi}_{l}\left(\mathbf{T}_{l}^{ \pm}\left(X_{0}\right), X_{0}\right)-D \boldsymbol{\Phi}_{L, l}\left(\mathbf{T}_{L, l}^{ \pm}\left(X_{0}\right), X_{0}\right)\right\|=\mathcal{O}\left(\sqrt{1-\left|Q_{0}\right|^{2} / c_{Q}^{2}} / E\right) .
\end{gathered}
$$

This does not only prove the estimate (4.19) on $\boldsymbol{\Psi}_{l}^{+}\left(\mathcal{H}_{l}^{>}\right) \cup \boldsymbol{\Psi}_{l}^{-}\left(\mathcal{H}_{l}^{>}\right) \subset \partial \mathcal{D}_{l}$, but also shows that $\boldsymbol{\Xi}$ is continuously differentiable at the submanifold

$$
\mathcal{H}_{l} \cap \partial \mathcal{D}_{l}=\boldsymbol{\Psi}_{l}^{+}\left(\mathcal{H}_{l}^{>}\right) \cap \boldsymbol{\Psi}_{l}^{-}\left(\mathcal{H}_{l}^{>}\right)
$$

of phase space points where the flows $\boldsymbol{\Phi}_{l}$ and $\boldsymbol{\Phi}_{L, l}$ are tangential to $\partial \mathcal{D}_{l}$ (for $X$ in this set $\Xi(X)=X$ and $D \Xi(X)=11)$.

2) • On $\mathcal{H}_{l}<$ we begin with a rough estimate. For time

$$
\begin{gathered}
\tau \equiv \tau\left(X_{0}\right):=\ln \left(3 c_{Q} /\left|X_{0}\right|\right) \\
\left|Q_{L}( \pm \tau)\right|=\left|\cosh (\tau) Q_{0}+\sinh (\tau) \tilde{P}_{0}\right|=\sqrt{\sinh ^{2}(\tau)\left|\tilde{P}_{0}\right|^{2}+\cosh ^{2}(\tau)\left|Q_{0}\right|^{2}} \\
\geq \sqrt{\frac{1}{2}\left|X_{0}\right|^{2} \cdot(\cosh (2 \tau)-1)} \geq \sqrt{\frac{1}{2}\left|X_{0}\right|^{2} \cdot\left(\frac{1}{2} \exp (2 \tau)-1\right)} \\
=\sqrt{\frac{9}{4} c_{q}-\frac{1}{2}\left|X_{0}\right|^{2}} \geq \frac{\sqrt{7}}{2} c_{Q}
\end{gathered}
$$

since by (4.7)

$$
\left|X_{0}\right|^{2}=2\left|Q_{0}\right|^{2}+\left(\left|\tilde{P}_{0}\right|^{2}-\left|Q_{0}\right|^{2}\right) \leq \frac{1}{2} c_{q}+\frac{Z_{l}-\left|Q_{0}\right|^{2} W_{l}\left(Q_{0}^{*} I_{3} Q_{0}\right)}{E} \leq c_{q} .
$$

Therefore any trajectory of the linear flow $\boldsymbol{\Phi}_{L, l}$ with these initial conditions leaves the region $\mathcal{D}_{l}$ before time $\tau$ (and enters it after time $-\tau$ ). 
- We now do perturbation theory around these linear solutions, setting

$$
Z(t):=X(t)-Y(t)
$$

$Z(t)$ meets the integral equation

$$
Z(t)=\int_{0}^{t}\left(\left(\begin{array}{ll}
0 & 11 \\
11 & 0
\end{array}\right) Z(s)+E^{-1} \tilde{R}(X(s))\right) d s
$$

with $E:=\mathcal{E}\left(X_{0}\right)$. Gronwall's inequality says that

$$
f(t) \leq A \exp \left(\int_{0}^{t} g(s) d s\right)
$$

if $f(t) \leq A+\int_{0}^{t} g(s) f(s) d s$. Applied to $f(t):=|Z(t)|$, we get for $0 \leq t \leq \mathbf{T}_{l}^{+}\left(X_{0}\right)$

$$
\begin{aligned}
f(t) & \leq \int_{0}^{t}\left(f(s)+E^{-1}|\tilde{R}(X(s))-\tilde{R}(Y(s))+\tilde{R}(Y(s))|\right) d s \\
& \leq E^{-1} \int_{0}^{t}|\tilde{R}(Y(s))| d s+\int_{0}^{t}\left(1+\frac{\mathcal{L}_{1}}{E}\right) f(s) d s \\
& \leq E^{-1} \mathcal{L}_{1} \int_{0}^{t}\left|Q_{L}(s)\right| d s+\int_{0}^{t}\left(1+\frac{\mathcal{L}_{1}}{E}\right) f(s) d s
\end{aligned}
$$

with the Lipschitz constant $\mathcal{L}_{1}$ from (4.25). But

$$
\begin{aligned}
\int_{0}^{t}\left|Q_{L}(s)\right| d s & =\int_{0}^{t}\left|\sinh (s) \tilde{P}_{0}+\cosh (s) Q_{0}\right| d s \\
& \leq\left|X_{0}\right| \int_{0}^{t} e^{s} d s \leq\left|X_{0}\right| e^{t}
\end{aligned}
$$

Thus the constants in (4.35) can be chosen as

$$
A \equiv A\left(X_{0}, t\right):=\frac{\mathcal{L}_{1}\left|X_{0}\right| e^{|t|}}{\mathcal{E}\left(X_{0}\right)} \quad, \quad g \equiv g\left(X_{0}\right):=1+\mathcal{L}_{1} / \mathcal{E}\left(X_{0}\right),
$$

and we obtain for $|t| \leq \tau\left(X_{0}\right)$ with $X(t) \in \mathcal{D}_{l}$

$$
\begin{aligned}
|Z(t)| \equiv & f(t) \leq \mathcal{L}_{1}\left|X_{0}\right| e^{|t|} \frac{\exp \left(\left(1+\mathcal{L}_{1} / E\right) \cdot \tau\left(X_{0}\right)\right)}{E} \\
& =\left|X_{0}\right| e^{|t|} \cdot \mathcal{O}\left(E^{-\frac{1}{2}}\right)=3 c_{Q} \cdot \mathcal{O}\left(E^{-\frac{1}{2}}\right)
\end{aligned}
$$

for $E_{\text {th }}$ large since by (4.33) and (4.26)

$$
\tau\left(X_{0}\right)=\ln \left(3 c_{Q} /\left|X_{0}\right|\right) \leq \ln \left(3 \sqrt{2} c_{Q} \sqrt{E /\left|Z_{l}\right|}\right)=\ln \left(c E^{\frac{1}{2}}\right) .
$$

In particular we conclude from (4.34) that

$$
0 \leq \pm \mathbf{T}_{l}^{ \pm}\left(X_{0}\right) \leq \tau\left(X_{0}\right),
$$


since otherwise by (4.37)

$$
|Q( \pm \tau)| \geq\left|Q_{L}( \pm \tau)\right|-|Z( \pm \tau)| \geq \frac{\sqrt{7}}{2} c_{Q}-3 c_{Q} \cdot \mathcal{O}\left(E^{-\frac{1}{2}}\right)>c_{Q} .
$$

We use (4.37) as an input for a refined estimate which will imply $|Z(t)|=$ $\mathcal{O}(1 / E)$. To that end we note that by (4.37)

$$
|Q(t)| \leq\left|Q_{L}(t)\right|+|Z(t)| \leq e^{|t|}\left|X_{0}\right|+|Z(t)| \leq 2 e^{|t|}\left|X_{0}\right| \quad\left(|t| \leq \tau\left(X_{0}\right)\right) .
$$

We write

$$
Z(t)=E^{-1} \int_{0}^{t} \exp \left(\left(\begin{array}{ll}
0 & \mathbb{1} \\
11 & 0
\end{array}\right)(t-s)\right) \tilde{R}(X(s)) d s .
$$

W.l.o.g. we may assume that $W_{l}\left(\vec{S}_{l}\right)=0$, since otherwise we may shift the energy $E$ by that constant, producing an error term of relative order $\mathcal{O}(1 / E)$. Then instead of the Lipschitz estimate (4.25) for $R$ we use

$$
R(Q) \leq \mathcal{L}_{2}|Q|^{2} \quad\left(|Q| \leq c_{Q}\right) .
$$

Inserting this into (4.38) we get for $0 \leq t \leq \tau\left(X_{0}\right)$, using (4.33),

$$
|Z(t)| \leq \frac{4 \mathcal{L}_{2}}{E} \int_{0}^{t} \exp (t-s) e^{2 s}\left|X_{0}\right|^{2} d s \leq \frac{12 \mathcal{L}_{2} c_{Q}}{E}\left(e^{t}-1\right)\left|X_{0}\right| \leq \frac{24 \mathcal{L}_{2}}{E}\left|Q_{L}(t)\right|,
$$

since

$$
\left|Q_{L}(t)\right|=\sqrt{\sinh ^{2}(t)\left|\tilde{P}_{0}\right|^{2}+\cosh ^{2}(t)\left|Q_{0}\right|^{2}} \geq \sinh (t)\left|X_{0}\right| .
$$

A similar estimate holds for $0 \geq t \geq-\tau\left(X_{0}\right)$.

But $|Q(t)| \geq\left|Q_{L}(t)\right|-|Z(t)|$, so that

$$
|Z(t)| \leq\left(\frac{E}{24 \mathcal{L}_{2}}-1\right)^{-1}|Q(t)|,
$$

showing that the diffeomorphism $\rho_{l}$ of (4.27) onto its image is $C^{0}$-near to the identity in the sense

$$
\frac{\left|\rho_{l}(X)-X\right|}{|Q|}=\mathcal{O}(1 / \mathcal{E}(X)) \quad\left(X=(\tilde{P}, Q) \in \mathcal{D}_{l}\right) .
$$

If we assume $X \in \partial \mathcal{D}_{l}$ so that $|Q|=c_{Q}$, then (4.39) shows that

$$
\left|Q_{L}\right|-c_{Q}=\mathcal{O}(1 / \mathcal{E}(X)) \text { for } \quad\left(\tilde{P}_{L}, Q_{L}\right):=\rho_{l}(X) .
$$

Since $X=\boldsymbol{\Psi}_{l}^{ \pm}\left(X_{0}\right)$ with $X_{0} \in \mathcal{H}_{l}^{<}$, and $\partial \mathcal{D}_{L, l}$ is uniformly transversal to the flow $\boldsymbol{\Phi}_{L, l}$ through $\mathcal{H}_{l}<$ we obtain from (4.40)

$$
\left|\mathbf{T}_{l}^{ \pm}\left(X_{0}\right)-\mathbf{T}_{L, l}^{ \pm}\left(X_{0}\right)\right|=\mathcal{O}\left(1 / \mathcal{E}\left(X_{0}\right)\right) \quad\left(X_{0} \in \mathcal{H}_{l}^{<}\right) .
$$

In turn, this and (4.39) imply (4.19). 
- We also get (4.17) from the time estimates (4.30) and (4.41) in the two regions.

- Estimate (4.18) for the exit time of the linear flow is derived as follows. By (4.11) the 'linear' energy parameter equals

$$
\mathcal{E}_{L}\left(X_{0}\right)=\frac{Z_{l}}{\left|\tilde{P}_{0}\right|^{2}-\left|Q_{0}\right|^{2}} .
$$

The (lifted) angular momentum (see (3.7) of the pericentric initial data $X_{0}=$ $\left(\tilde{P}_{0}, Q_{0}\right)$ equals

$$
\begin{aligned}
& \left|\vec{L}\left(X_{0}\right)\right|= \\
& \quad \frac{1}{4}\left|Q_{0}^{*} P_{0}-P_{0}^{*} Q_{0}\right|=\frac{1}{2}\left|\operatorname{Im}\left(Q_{0}^{*} P_{0}\right)\right|=\frac{1}{2}\left|P_{0}\right|\left|Q_{0}\right|=\sqrt{2 \mathcal{E}_{L}\left(X_{0}\right)}\left|\tilde{P}_{0}\right|\left|Q_{0}\right|,
\end{aligned}
$$

since $\left\langle P_{0}, Q_{0}\right\rangle=0$, see (3.7). Thus

$$
\frac{\left|Z_{l}\right| \cdot e\left(X_{0}\right)}{\mathcal{E}_{L}\left(X_{0}\right)}=\sqrt{\left(Z_{l} / \mathcal{E}_{L}\left(X_{0}\right)\right)^{2}+4\left|\tilde{P}_{0}\right|^{2}\left|Q_{0}\right|^{2}}=\left|\tilde{P}_{0}\right|^{2}+\left|Q_{0}\right|^{2} .
$$

On the other hand the exit times $\mathbf{T}_{L, l}^{+}\left(X_{0}\right)$ and $\mathbf{T}_{L, l}^{-}\left(X_{0}\right)=-\mathbf{T}_{L, l}^{+}\left(X_{0}\right)$ are implicitly given by the equation

$$
\left|Q_{L}\left(\mathbf{T}_{L, l}^{+}\left(X_{0}\right), X_{0}\right)\right|=c_{Q}
$$

with $Q_{L}\left(t, X_{0}\right)=\sinh (t) \tilde{P}_{0}+\cosh (t) Q_{0}$, whence

$$
\begin{aligned}
\exp \left(2 \mathbf{T}_{L, l}^{+}\left(X_{0}\right)\right) & =\frac{4 c_{Q}^{2}}{\left|\tilde{P}_{0}\right|^{2}+\left|Q_{0}\right|^{2}}+\mathcal{O}\left(\mathcal{E}_{L}^{0}\left(X_{0}\right)\right) \\
& =\frac{4 c_{q} \mathcal{E}_{L}\left(X_{0}\right)}{\left|Z_{l}\right| e\left(X_{0}\right)}+\mathcal{O}\left(\mathcal{E}_{L}^{0}\left(X_{0}\right)\right) .
\end{aligned}
$$

This proves (4.18).

- The $C^{0}$-estimate (4.20) follows immediately from (4.19).

- In order to obtain the $C^{1}$-estimate (4.21), one considers

$$
D Z(t):=D \boldsymbol{\Phi}_{l}^{t}(X)-D \boldsymbol{\Phi}_{L, l}^{t}(\boldsymbol{\Xi}(X)) \quad\left(X_{0} \in \boldsymbol{\Psi}_{l}^{ \pm}\left(\mathcal{H}_{l}^{<}\right)\right) .
$$

$D Z(t)$ solves the integral equation

$$
D Z(t)=\int_{0}^{t}\left(\left(\begin{array}{ll}
0 & 11 \\
11 & 0
\end{array}\right) D Z(s)+E^{-1} D \tilde{R}(X(s)) D X(s)\right) d s .
$$

W.l.o.g. we may again assume that $W_{l}\left(\vec{s}_{l}\right)=0$, since otherwise we may shift the energy $E$ by that constant, producing an error term of relative order $\mathcal{O}(1 / E)$. Then by inspection of $(4.5)$ one notes that the matrix $D \tilde{R}((\tilde{P}, Q))=0$ at $Q=0$. We may thus estimate

$$
\|D \tilde{R}((\tilde{P}, Q))\| \leq \mathcal{L}_{3}|Q| \quad\left((\tilde{P}, Q) \in \mathcal{D}_{l}\right),
$$

where $\mathcal{L}_{3}>0$ is the Lipschitz constant of $D R$ for $|Q| \leq c_{Q}$. 
From (4.42) we obtain (4.21) by a Gronwall estimate similar to (4.37), which we apply to

$$
D Z(t)=\int_{0}^{t}\left[\left(\left(\begin{array}{ll}
0 & 1 \\
11 & 0
\end{array}\right)+E^{-1} D \tilde{R}(X(s))\right) D Z(s)+E^{-1} D \tilde{R}(X(s)) D Y(s)\right] d s,
$$

with $t \leq \mathbf{T}_{l}^{+}\left(X_{0}\right), f(t):=\|D Z(t)\|$,

$$
g(s):=1+\mathcal{L}_{3} c_{Q} / E \geq\left\|\left(\begin{array}{ll}
0 & \mathbb{1 1} \\
\mathbb{1} & 0
\end{array}\right)+E^{-1} D \tilde{R}(X(s))\right\|
$$

and

$$
A:=\frac{4 c_{q} \mathcal{L}_{3}}{\sqrt{\left|Z_{l}\right|}} \sqrt{E / e} \geq E^{-1}\left\|\int_{0}^{\mathbf{T}_{l}^{+}\left(X_{0}\right)} D \tilde{R}(X(s)) D Y(s) d s\right\| .
$$

To obtain the last estimate, the time bounds (4.17) and (4.18) are inserted. Thus for $t \leq \mathbf{T}_{l}^{+}\left(X_{0}\right)$

$$
f(t) \leq A \exp \left(\int_{0}^{t} g(s) d s\right)=\mathcal{O}\left(E^{-\frac{1}{2}} e\left(X_{0}\right)^{-\frac{1}{2}}\right) \cdot \mathcal{O}\left(E^{\frac{1}{2}} e\left(X_{0}\right)^{-\frac{1}{2}}\right) .
$$

\section{Regularization by phase space extension}

In Sect. 4 the motion in configuration space $\mathbb{R}_{\vec{q}}^{3}$ near a singularity at $\vec{s}_{l}$ was regularized using the KS transform. This will enable us in Sect. 8 to compare that motion with the motion in the Kepler potential $\vec{q} \mapsto-Z_{l} /\left|\vec{q}-\vec{s}_{l}\right|$.

However, we would like to apply these local estimates to a complete Hamiltonian flow on a phase space which arises by a completion of $T^{*} \hat{M}$. Therefore in the case of attracting singularities $\left(Z_{l}>0\right)$ we now employ a different regularization.

To preserve continuity of the motion with respect to the initial conditions, a particle colliding with a nucleus at $\vec{s}_{l} \in \mathbb{R}_{\vec{q}}^{3}$ must be reflected backwards. Then we parametrize the state of the colliding particle by its energy and by its incoming (or outgoing) direction. That is, we complete phase space by adjoining manifolds $\mathbb{R} \times S^{2}$, one for each attracting singularity.

Note that for all energies $E$ the energy surfaces $\hat{H}^{-1}(E)$ could be completed topologically using only the Kustaanheimo-Stiefel construction (by taking the quotient of the quadric surface $\mathcal{I}^{-1}(0)$ w.r.t. the circle action $\alpha$ defined in (3.5)). This, however would lead to a time change which is unwanted here.

Theorem 5.1. There exists a unique smooth extension $(P, \omega, H)$ of the Hamiltonian system $\left(T^{*} \hat{M}, \hat{\omega}, \hat{H}\right)$, where the phase space $P$ is a smooth six-dimensional manifold with

$$
P:=T^{*} \hat{M} \cup \bigcup_{\substack{1 \leq l \leq n \\ Z_{l}>0}}\left(\mathbb{R} \times S^{2}\right)
$$


as a set, $\omega$ is a smooth symplectic two-form on $P$ with

$$
\omega \uparrow_{T^{*} \hat{M}}=\hat{\omega}:=\sum_{i=1}^{3} d q_{i} \wedge d p_{i},
$$

and $H: P \rightarrow \mathbb{R}$ is a smooth Hamiltonian function with $H \uparrow_{T^{*} \hat{M}}=\hat{H}$.

The smooth Hamiltonian flow

$$
\Phi: \mathbb{R} \times P \rightarrow P
$$

generated by $H$ is complete (and we often write $\Phi^{t}(x)$ instead of $\Phi(t, x)$ ).

For all energies $E>V_{\max }$ (defined in (2.9)) the energy shell

$$
\Sigma_{E}:=\{x \in P \mid H(x)=E\}
$$

is a smooth, five-dimensional manifold.

Proof. It is clear that the particle cannot escape to spatial infinity in finite time. If $Z_{l}<0$ there is no need here to regularize, since then by (4.2) the minimal distance is bounded by $\left|\vec{q}-\vec{s}_{l}\right| \geq\left|Z_{l}\right| /(2 E)$ if $E>0$ resp. $\left|\vec{q}-\vec{s}_{l}\right| \geq c_{q}$ if $E \leq 0$.

For the remaining case of an attracting singularity $\left(Z_{l}>0\right)$ we linearize the motion near collision by using as phase space coordinates the angular momentum components, the direction of the Runge-Lenz vector $\vec{F}_{l}$, energy and the time passed since the pericentre of the orbit. The first five of these six functions are constant on the Kepler orbit. Then we add the collision manifold of phase space points with time and angular momentum both equal to zero. This manifold is then parametrized by energy and by $\vec{F}_{l} /\left|\vec{F}_{l}\right|$ and is thus diffeomorphic to $\mathbb{R} \times S^{2}$.

In $[\mathrm{KK}]$ the case of $d=2$ dimensions is treated, the calculations being more detailed than here. Comparing with the Delaunay coordinates (see, e.g., [AM], Chapter 9.3), instead of the semi-major axis we use energy, and instead of the mean anomaly (which is only defined for negative energies) we use time.

If the potential $V$ is not centrally symmetric around $\vec{s}_{l}$, then at collision some of the former constants of motion cease to be smooth functions of time. To remedy this is we redefine them by using their value at the pericentre of the orbit.

1) More specifically, we introduce adapted coordinates to regularize the flow in the phase space neighbourhood $\hat{U}_{l}^{\varepsilon}, 0<\varepsilon \leq c_{q}$, of the $l$ th nucleus, with

$$
\hat{U}_{l}^{\varepsilon}:=\left\{(\vec{p}, \vec{q}) \in T^{*} \hat{M}|| \vec{q}-\left.\vec{s}_{l}|<\varepsilon,| \vec{p}\right|^{2}>\frac{3}{2} \frac{Z_{l}}{\left|\vec{q}-\vec{s}_{l}\right|}\right\} .
$$

On $\hat{U}_{l}^{\varepsilon}$, the Hamiltonian function has the form $\hat{H}(\vec{p}, \vec{q})=\hat{H}_{l}(\vec{p}, \vec{q})+W_{l}(\vec{q})$ with

$$
\hat{H}_{l}(\vec{p}, \vec{q}):=\frac{1}{2} \vec{p}^{2}-\frac{Z_{l}}{\left|\vec{q}-\vec{s}_{l}\right|}
$$

and the smooth additional potential $W_{l}$ on $B_{l}\left(c_{q}\right)$,

$$
W_{l}(\vec{q})=\sum_{i \neq l} \frac{-Z_{i}}{\left|\vec{q}-\vec{s}_{i}\right|}+W(\vec{q}) .
$$


One basic estimate on $\hat{U}_{l}^{\varepsilon}$, valid for $\varepsilon$ small, is

$$
\frac{d}{d t}\left(\left(\vec{q}-\vec{s}_{l}\right) \cdot \vec{p}\right) \geq \frac{1}{2} \frac{Z_{l}}{\left|\vec{q}-\vec{s}_{l}\right|}-\left(\vec{q}-\vec{s}_{l}\right) \cdot \nabla W_{l}(\vec{q})>0 .
$$

Every collision orbit with $\vec{s}_{l}$ enters $\hat{U}_{l}^{\varepsilon}$, as

$$
|\vec{p}|^{2}-\frac{3}{2} \frac{Z_{l}}{\left|\vec{q}-\vec{s}_{l}\right|}=\frac{1}{2} \frac{Z_{l}}{\left|\vec{q}-\vec{s}_{l}\right|}+2\left(E-W_{l}(\vec{q})\right) \rightarrow \infty
$$

as $\vec{q}$ approaches $\vec{s}_{l}$.

2) We first treat the Keplerian case $W_{l} \equiv 0$. The angular momentum $\hat{L}_{l}: \hat{U}_{l}^{\varepsilon} \rightarrow \mathbb{R}^{3}$ relative to the position of the $l$ th nucleus equals

$$
\hat{L}_{l}(\vec{p}, \vec{q}):=\left(\vec{q}-\vec{s}_{l}\right) \times \vec{p} .
$$

Let $\hat{T}_{l}: \hat{U}_{l}^{\varepsilon} \rightarrow \mathbb{R}$ be the time elapsed since the closest encounter of the Kepler solution with the nucleus. By (5.5) there is only one such pericentre of the orbit, with distance $r_{\min } . \hat{T}_{l}$ is given by

$$
\hat{T}_{l}(\vec{p}, \vec{q}):=\int_{r_{\min }(\vec{p}, \vec{q})}^{\left|\vec{q}-\vec{s}_{l}\right|} \frac{r d r}{\sqrt{2 r^{2} \hat{H}_{l}(\vec{p}, \vec{q})+2 Z_{l} r-\hat{L}_{l}^{2}(\vec{p}, \vec{q})}} \cdot \operatorname{sign}\left(\left(\vec{q}-\vec{s}_{l}\right) \cdot \vec{p}\right)
$$

with

$$
r_{\min }(\vec{p}, \vec{q}):=\left\{\begin{array}{cc}
\frac{-Z_{l}+\sqrt{Z_{l}^{2}+2 \hat{H}_{l}(\vec{p}, \vec{q}) \hat{L}_{l}^{2}(\vec{p}, \vec{q})}}{2 \hat{H}_{l}(\vec{p}, \vec{q})}, \hat{H}_{l} \neq 0 \\
\hat{L}_{l}^{2}(\vec{p}, \vec{q}) / 2 Z_{l} & , \hat{H}_{l}=0
\end{array}\right.
$$

$\hat{T}_{l}$ is a smooth function, which can be seen by explicit evaluation of the integrals:

$$
\begin{aligned}
& \int \frac{r}{\sqrt{2 r^{2} E+2 Z r-L^{2}}} d r= \\
& \frac{r}{\sqrt{2 E}} \sqrt{1+\frac{Z}{r E}-\frac{L^{2}}{2 r^{2} E}}-\frac{Z}{(2 E)^{3 / 2}} \ln \left(E r+\frac{1}{2} Z+\sqrt{E\left(r^{2} E+Z r-\frac{1}{2} L^{2}\right)}\right)
\end{aligned}
$$

for $E>0$ and $Z>0$ (see Thirring [Th], for more information).

The Runge-Lenz vector $\vec{F}_{l}: \hat{U}_{l}^{\varepsilon} \rightarrow \mathbb{R}^{3}$ relative to the $l$ th centre is given by

$$
\vec{F}_{l}(\vec{p}, \vec{q}):=\vec{p} \times \hat{L}_{l}(\vec{p}, \vec{q})-Z_{l} \frac{\vec{q}-\vec{s}_{l}}{\left|\vec{q}-\vec{s}_{l}\right|} .
$$

On its domain $\hat{U}_{l}^{\varepsilon}$ of definition $\vec{F}_{l}$ is non-zero: $\left|\vec{F}_{l}\right|^{2}=2\left|\hat{L}_{l}\right|^{2} \hat{H}_{l}+Z_{l}^{2}>Z_{l}^{2} / 4$. Thus we may define the pericentral direction $\hat{F}_{l}: \hat{U}_{l}^{\varepsilon} \rightarrow S^{2}$ by $\hat{F}_{l}:=\vec{F}_{l} /\left|\vec{F}_{l}\right|$. The angular momentum vector $\hat{L}_{l}$ is perpendicular to that direction: $\hat{L}_{l} \cdot \hat{F}_{l}=0$. The map

$$
\hat{\mathcal{Y}}: \hat{U}_{l}^{\varepsilon} \rightarrow T^{*}\left(\mathbb{R} \times S^{2}\right) \backslash \overline{0}, \quad(\vec{p}, \vec{q}) \mapsto\left(\hat{T}_{l}, \hat{L}_{l} ; \hat{H}_{l}, \hat{F}_{l}\right)
$$


is a diffeomorphism onto its image, $(\overline{0}$ denoting the zero section of the cotangent bundle $T^{*}\left(\mathbb{R} \times S^{2}\right)$ ).

3) The Poisson brackets between the above variables are given by

$$
\left\{\hat{T}_{l}, \hat{H}_{l}\right\}=1,\left\{\hat{L}_{l}, \hat{H}_{l}\right\}=\overrightarrow{0},\left\{\hat{F}_{l}, \hat{H}_{l}\right\}=\overrightarrow{0},\left\{\hat{F}_{l}, \hat{T}_{l}\right\}=\overrightarrow{0} \text { and }\left\{\hat{L}_{l}, \hat{T}_{l}\right\}=\overrightarrow{0},
$$

so that in particular the angular momentum and the pericentral direction are constants of the Kepler flow. The components of angular momentum and the asymptotic direction have the Poisson brackets

$$
\left\{\left(\hat{F}_{l}\right)_{i},\left(\hat{F}_{l}\right)_{j}\right\}=0, \quad\left\{\left(\hat{L}_{l}\right)_{i},\left(\hat{L}_{l}\right)_{j}\right\}=\varepsilon_{i j k}\left(\hat{L}_{l}\right)_{k}, \quad\left\{\left(\hat{L}_{l}\right)_{i},\left(\hat{F}_{l}\right)_{j}\right\}=\varepsilon_{i j k}\left(\hat{F}_{l}\right)_{k},
$$

using the Poisson brackets $\left\{\left(\vec{F}_{l}\right)_{i},\left(\vec{F}_{l}\right)_{j}\right\}=-2 \hat{H}_{l} \varepsilon_{i j k}\left(\vec{F}_{l}\right)_{k}$.

4) Because of the above Poisson brackets with $\hat{H}_{l}$, by introducing the above coordinates, we obtain a chart in $\hat{U}_{l}^{\varepsilon}$ which explicitly linearizes the (incomplete) Kepler flow.

The motion is then regularized in the following way. One defines a completion of $\hat{U}_{l}^{\varepsilon}$ by setting $U_{l}^{\varepsilon}:=\hat{U}_{l}^{\varepsilon} \cup\left(\mathbb{R} \times S^{2}\right)$ as a set, and one introduces a topology on $U_{l}^{\varepsilon}$ by extending the map $\hat{\mathcal{Y}}=\left(\hat{T}_{l}, \hat{L}_{l} ; \hat{H}_{l}, \hat{F}_{l}\right)$ to

$$
\mathcal{Y}:=\left(T_{l}, \mathbf{L}_{l} ; H_{l}, \mathbf{F}_{l}\right): U_{l}^{\varepsilon} \rightarrow T^{*}\left(\mathbb{R} \times S^{2}\right)
$$

by mapping $(h, \mathbf{f}) \in \mathbb{R} \times S^{2}$ onto the point $(0,0 ; h, \mathbf{f})$ of the zero section. By that procedure we obtain the topological manifold $P$ and, by taking limits, we extend the Hamiltonian $\hat{H}$ to a continuous function $H: P \rightarrow \mathbb{R}$. The topology of $P$ is thus determined by the purely Coulombic local Hamiltonians $\hat{H}_{l}$, and, by taking limits for the collision orbits, we are able to extend the flow generated by $\hat{H}$ to a complete continuous flow $\Phi^{t}$ on $P$.

Moreover, the calculation of all the Poisson brackets shows that we may continuously extend the symplectic form $\left.\left(\sum_{i=1}^{3} d q_{i} \wedge d p_{i}\right)\right|_{\hat{U}_{l}^{\varepsilon}}$ to $U_{l}^{\varepsilon}$ and obtain a nondegenerate two-form, which is smooth in the new coordinates.

5) To generalize the construction to the case of the flow generated by $H$ which is of the local form $H_{l}+W_{l}$, we define similar canonical coordinates in $U_{l}^{\varepsilon}$ which linearize the $H$-flow $\Phi^{t}$.

By (5.5) for $\varepsilon>0$ small enough, the orbits are transversal to the hypersurface $\hat{S}_{l}^{\varepsilon}$. We extend that hypersurface to the topological submanifold

$$
S_{l}^{\varepsilon}:=\hat{S}_{l}^{\varepsilon} \cup\left(\mathbb{R} \times S^{2}\right) \subset U_{l}^{\varepsilon} .
$$

If $W_{l} \equiv 0$, we define the differential structure near the $l$ th collision manifold $\mathbb{R} \times S^{2}$ by pull-back with $\mathcal{Y}$. In general we define a map

$$
\tilde{\mathcal{Y}}:=\left(\tilde{T}_{l}, \tilde{L}_{l} ; \tilde{H}_{l}, \tilde{F}_{l}\right): U_{l}^{\varepsilon} \rightarrow T^{*}\left(\mathbb{R} \times S^{2}\right)
$$

by letting $\tilde{H}_{l}:=\left.H\right|_{U_{l}^{\varepsilon}}$ be the energy and

$$
\tilde{T}_{l}\left(\Phi^{t}(x)\right):=t \quad\left(x \in S_{l}^{\varepsilon}\right)
$$


the time passed since the passage of the pericentre. Note that (5.12) defines $\tilde{T}_{l}$ everywhere on $U_{l}^{\varepsilon}$, since every orbit in $U_{l}^{\varepsilon}$ passes $S_{l}^{\varepsilon}$ exactly once.

$$
\tilde{L}_{l}\left(\Phi^{t}(x)\right):=\mathbf{L}_{l}(x) \quad \text { and } \quad \tilde{F}_{l}\left(\Phi^{t}(x)\right):=\mathbf{F}_{l}(x) \quad\left(x \in S_{l}^{\varepsilon}\right)
$$

are then the angular momentum and the asymptotic direction at the pericentre $x$ of the orbit.

Clearly, by fiat, $\tilde{H}_{l}, \tilde{L}_{l}$ and $\tilde{F}_{l}$ are constant on one orbit $\Phi^{t}(\vec{p}, \vec{q})$, whereas $\tilde{T}_{l}\left(\Phi^{t}(\vec{p}, \vec{q})\right)$ is an affine function of time $t$. Therefore we have linearized the full motion. We must show that the functions $\left(\tilde{T}_{l}, \tilde{L}_{l} ; \tilde{H}_{l}, \tilde{F}_{l}\right)$, restricted to $\hat{U}_{l}^{\varepsilon}$, are indeed smooth coordinates. But this follows from the smoothness of the lifted functions in Lemma 3.1, smoothness of time change, and smoothness of the KS flow (4.6) (which of course exists for all energies $E \in \mathbb{R}$ ).

Since $\tilde{\mathcal{Y}} \equiv\left(\tilde{T}_{l}, \tilde{L}_{l} ; \tilde{H}_{l}, \tilde{F}_{l}\right)$ defines a homeomorphism of $U_{l}^{\varepsilon}$ onto its image, we use it to define a differential structure on the whole of $U_{l}^{\varepsilon}$ and thus on $P$.

Smoothness of the energy shells $\Sigma_{E}$ for $E>V_{\max }$ follows by noticing that these $E$ are regular values of $H: P \rightarrow \mathbb{R}$.

\section{Møller transformations}

Next we define the Møller and scattering transformations which compare the asymptotics of the motion with a 'free motion'. We base ourselves on the articles [Hu] of Hunziker and [Sim] of Simon. The recent monograph [DG] by Dereziński and Gérard treats these questions in the context of classical and quantum mechanical $n$-body scattering.

Due to the long-range character of the Coulomb interaction we cannot in general use the flow generated by the Hamiltonian function $\vec{p}^{2} / 2$ as 'free motion'. Instead, we compare with the Kepler motion generated by

$$
\hat{H}_{\infty}(\vec{p}, \vec{q}):=\frac{1}{2} \vec{p}^{2}+V_{\infty}(\vec{q}) \quad \text { with } \quad V_{\infty}(\vec{q})=-\frac{Z_{\infty}}{|\vec{q}|} .
$$

Thus we consider the smooth complete flow

$$
\Phi_{\infty}^{t}: P_{\infty} \rightarrow P_{\infty}
$$

generated by (6.1).

- If $Z_{\infty}=0, \Phi_{\infty}^{t}$ is the free flow on the phase space $P_{\infty}:=T^{*} \mathbb{R}^{3}$.

- If $Z_{\infty}>0$, we regularize $T^{*}\left(\mathbb{R}^{3} \backslash\{0\}\right)$ in the way described in Theorem 5.1 to obtain $P_{\infty}$.

- If $Z_{\infty}<0$, then $\Phi_{\infty}^{t}$ is already complete on $P_{\infty}:=T^{*}\left(\mathbb{R}^{3} \backslash\{0\}\right)$, since particles of finite energy cannot meet the origin at $\vec{q}=\overrightarrow{0}$.

Thus we are to compare motions $\Phi^{t}$ and $\Phi_{\infty}^{t}$ on the different phase spaces $P$ and $P_{\infty}$. We cannot just identify $P$ with $P_{\infty}$ by neglecting the measure zero sets projecting to the singularities, since later on we will be interested in certain sets of measure zero like the bound states (moreover, these bound states will turn out to be crucial in our analysis of scattering, too). 
We overcome the above difficulty by observing that it suffices to identify $P_{\infty}$ with $P$ in a neighbourhood of spatial infinity. More precisely, let

$$
P_{\infty,+}:=\left\{x \in P_{\infty} \mid H_{\infty}(x)>0\right\}
$$

be the set of phase space points with positive 'free' energy. Then the orbit $\Phi_{\infty}^{t}(x)$ starting at $x \in P_{\infty,+}$ goes to spatial infinity for large positive and negative times. The ball $\left\{\vec{q} \in \mathbb{R}_{\vec{q}}^{3}|| \vec{q} \mid \leq R_{\min }\right\}$ contains all singularities of $V$ and the singularity at the origin of the Kepler Hamiltonian $H_{\infty}$. Therefore, we can canonically identify points $(\vec{p}, \vec{q}) \in P_{\infty}$ with points $(\vec{p}, \vec{q}) \in P$ if $|\vec{q}|>R_{\min }$, and we denote this identification by Id. Thus the Mфller transformations

$$
\Omega^{ \pm}:=\lim _{t \rightarrow \pm \infty} \Phi^{-t} \circ \operatorname{Id} \circ \Phi_{\infty}^{t}
$$

are formally maps $\Omega^{ \pm}: P_{\infty,+} \rightarrow P$. They exist as pointwise limits, see Theorem 6.3 below.

First some standard definitions (see $[\mathrm{Hu}]$ ):

\section{Definition 6.1.}

$$
\begin{aligned}
& b^{ \pm}:=\left\{x \in P \mid \vec{q}\left( \pm \mathbb{R}^{+}, x\right) \text { is bounded }\right\} \quad, \quad b_{E}^{ \pm}:=b^{ \pm} \cap \Sigma_{E} \\
& b:=b^{+} \cap b^{-} \quad \text { (the bound states) } \quad, \quad b_{E}:=b \cap \Sigma_{E} \\
& s^{ \pm}:=\left\{x \in P \mid x \notin b^{ \pm} \text {and } H(x)>0\right\} \quad, \quad s_{E}^{ \pm}:=s^{ \pm} \cap \Sigma_{E} \\
& s:=s^{+} \cap s^{-} \quad \text { (the scattering states) } \quad, s_{E}:=s \cap \Sigma_{E} \text {. }
\end{aligned}
$$

We shall show that $s^{ \pm}=\Omega^{ \pm}\left(P_{\infty,+}\right)$ so that the term 'scattering states' is really justified.

Remarks 6.2. 1) By continuity of $\Phi^{t}, b^{ \pm}$can be represented as the union $b^{ \pm}=$ $\cup_{k=1}^{\infty} b^{ \pm, k}$ of compact sets $b^{ \pm, k}$. Hence $b^{ \pm}$and $s^{ \pm}$are measurable w.r.t. Liouville measure

$$
\lambda:=\frac{1}{3 !} \omega \wedge \omega \wedge \omega
$$

on the symplectic manifold $(P, \omega)$.

2) The sets $b^{ \pm}$and $s^{ \pm}$are also $\Phi^{t}$-invariant.

For all $E>0$ the sets $b_{E}^{ \pm}$are closed, and $b_{E}$ is compact, being a subset of the compact region in $\Sigma_{E}$ projecting to the ball $\left\{\vec{q} \in \mathbb{R}^{3}|| \vec{q} \mid \leq R_{\mathrm{vir}}(E)\right\}$ in configuration space.

Theorem 6.3. The limits

$$
\Omega^{ \pm}=\lim _{t \rightarrow \pm \infty} \Phi^{-t} \circ \operatorname{Id} \circ \Phi_{\infty}^{t}
$$

exist pointwisely on $P_{\infty,+} \subset P_{\infty}$ and thus define the Moller transformations $\Omega^{ \pm}: P_{\infty,+} \rightarrow s^{ \pm}$. These are measure-preserving homeomorphisms and intertwine $\Phi^{t}$ and $\Phi_{\infty}^{t}$ :

$$
\Omega^{ \pm} \circ \Phi_{\infty}^{t}=\Phi^{t} \circ \Omega^{ \pm} .
$$


The asymptotic limits $\vec{p}^{ \pm}: s^{ \pm} \rightarrow \mathbb{R}^{3}$ and $\vec{L}^{ \pm}: s^{ \pm} \rightarrow \mathbb{R}^{3}$ of the momentum and the angular momentum $\vec{L}(\vec{p}, \vec{q}):=\vec{q} \times \vec{p}$

$$
\vec{p}^{ \pm}\left(x_{0}\right):=\lim _{t \rightarrow \pm \infty} \vec{p} \circ \Phi^{t}\left(x_{0}\right) \quad \text { and } \quad \vec{L}^{ \pm}\left(x_{0}\right):=\lim _{t \rightarrow \pm \infty} \vec{L} \circ \Phi^{t}\left(x_{0}\right)
$$

are continuous functions. If $\left(\vec{p}_{0}, \vec{q}_{0}\right) \equiv x_{0}$ with $q_{0}:=\left|\vec{q}_{0}\right|>R_{\mathrm{vir}}(E)$ and $\pm\left\langle\vec{q}_{0}, \vec{p}_{0}\right\rangle \geq 0$, then

$$
\vec{p}^{ \pm}\left(x_{0}\right)=\vec{p}_{0}+\mathcal{O}\left(1 /\left(q_{0} \sqrt{E}\right)\right) \quad, \quad \vec{L}^{ \pm}\left(x_{0}\right)=\vec{L}\left(x_{0}\right)+\mathcal{O}\left(1 /\left(q_{0}^{\epsilon} \sqrt{E}\right)\right),
$$

and for $\left(\vec{P}_{0}, \vec{Q}_{0}\right):=\Omega^{ \pm}\left(\vec{p}_{0}, \vec{q}_{0}\right)$ and $E>E_{\text {th }}$

$$
\vec{P}_{0}=\vec{p}_{0}+\mathcal{O}\left(1 /\left(q_{0}^{1+\epsilon} \sqrt{E}\right)\right) \quad, \quad \vec{Q}_{0}=\vec{q}_{0}+\mathcal{O}\left(1 /\left(q_{0}^{\epsilon} E\right)\right) .
$$

If $\epsilon=1$ in Definition 2.1 of Coulombic potentials, then for all energies $E>0$

$$
\left|\vec{P}_{0}-\vec{p}_{0}\right| \leq \frac{\sqrt{E} R_{\min }}{q_{0}} \frac{C_{2}}{E q_{0}} \quad, \quad\left|\vec{Q}_{0}-\vec{q}_{0}\right| \leq R_{\min } \frac{C_{2}}{E q_{0}}
$$

(remark that by Definition (2.14) of $C_{2}$ one always has $\frac{C_{2}}{E q_{0}} \leq 1$ ).

Proof. First we show (6.5). Since the system is reversible, we consider only the case $t \rightarrow+\infty$. For initial conditions $x_{0} \in s_{E}^{+}$there exists a time $t_{0}$ with

$$
\left|\vec{q}\left(t_{0}\right)\right| \geq R_{\mathrm{vir}}(E) \quad \text { and } \quad\left\langle\vec{q}\left(t_{0}\right), \vec{p}\left(t_{0}\right)\right\rangle \geq 0
$$

for $(\vec{p}(t), \vec{q}(t)):=\Phi^{t}\left(x_{0}\right)$, since otherwise $|\vec{q}(t)|$ would be uniformly bounded as $t \rightarrow \infty$.

W.l.o.g. we assume $t_{0}=0$. Then by (2.16)

$$
\vec{q}^{2}(t) \geq \vec{q}_{0}^{2}+\frac{1}{2} E t^{2} \quad(t \geq 0) .
$$

Thus by (2.5)

$$
\left|\frac{d}{d t} \vec{p}(t)\right|=|\nabla V(\vec{q}(t))| \leq \frac{\left|Z_{\infty}\right|+C_{1} R_{\min }^{1-\epsilon}}{q_{0}^{2}+\frac{1}{2} E t^{2}} \quad(t \geq 0),
$$

the limit $\vec{p}^{+}\left(x_{0}\right)=\lim _{t \rightarrow \infty} \vec{p}(t)$ exists, and for this choice of $x_{0}$

$$
\vec{p}^{+}\left(x_{0}\right)-\vec{p}_{0}=\mathcal{O}\left(1 /\left(q_{0} \sqrt{E}\right)\right) .
$$

Being a locally uniform limit of the continuous functions $x_{0} \mapsto \vec{p}\left(t, x_{0}\right), \vec{p}^{+}$ is continuous. Next we show that the asymptotic limit $\vec{L}^{+}\left(x_{0}\right)$ of the angular momentum exists. For $t \geq 0$ we can estimate

$$
\begin{aligned}
\left|\frac{d}{d t} \vec{L}(\vec{p}(t), \vec{q}(t))\right| & =|\nabla V(\vec{q}(t)) \times \vec{q}(t)| \\
& =\left|\left(\nabla V(\vec{q}(t))-Z_{\infty} \frac{\vec{q}(t)}{|\vec{q}(t)|^{3}}\right) \times \vec{q}(t)\right| \\
& \leq C_{1} R_{\min }|\vec{q}(t)|^{-1-\epsilon} \leq C_{1} R_{\min }\left(q_{0}^{2}+\frac{1}{2} E t^{2}\right)^{-\frac{1}{2}(1+\epsilon)}
\end{aligned}
$$


using (2.5) and (2.16), which shows the existence of the limit $\vec{L}^{+}\left(x_{0}\right)$, and (6.6). Continuity of $\vec{L}^{+}$follows as above.

We now seek a Kepler hyperbola $(\vec{P}(t), \vec{Q}(t)):=\Phi_{\infty}^{t}\left(X_{0}\right)$ which is positive asymptotic to $(\vec{p}(t), \vec{q}(t))$ and write

$$
\vec{r}(t):=\vec{q}(t)-\vec{Q}(t) .
$$

Then $\vec{r}$ is a solution of the differential equation

$$
\ddot{\vec{r}}(t)=Z_{\infty} \frac{\vec{q}(t)-\vec{r}(t)}{|\vec{q}(t)-\vec{r}(t)|^{3}}-\nabla V(\vec{q}(t)) \quad \text { with } \quad \lim _{t \rightarrow \infty} \vec{r}(t)=\overrightarrow{0} .
$$

Setting for $E>0$

$$
\mathcal{C}_{E}:=\left\{\vec{r} \in C\left([0, \infty), \mathbb{R}^{3}\right)\left|\|\vec{r}\|:=\sup _{t \geq 0}\right| \vec{r}(t) \mid<\min \left(R_{\min }, C_{2} / E\right)\right\}
$$

(with $C_{2}=31(1+1 / \epsilon) R_{\min }^{1-\epsilon} C_{1}$ from (2.14)), by (2.16) and (2.14)

$$
(\mathcal{F} \vec{r})(t):=\int_{t}^{\infty} d s \int_{s}^{\infty} d \tau\left(Z_{\infty} \frac{\vec{q}(\tau)-\vec{r}(\tau)}{|\vec{q}(\tau)-\vec{r}(\tau)|^{3}}-\nabla V(\vec{q}(\tau))\right)
$$

is well-defined for $\vec{r} \in \mathcal{C}_{E}$, noting that by (2.14)

$$
|\vec{q}(\tau)-\vec{r}(\tau)| \geq R_{\min } .
$$

We estimate $|(\mathcal{F} \vec{r})(t)|$ as follows. The integrand of (6.10) is bounded by

$$
\begin{aligned}
& \left|Z_{\infty} \frac{\vec{q}(\tau)-\vec{r}(\tau)}{|\vec{q}(\tau)-\vec{r}(\tau)|^{3}}-\nabla V(\vec{q}(\tau))\right| \\
& \leq\left|\nabla V(\vec{q}(\tau))-Z_{\infty} \frac{\vec{q}(\tau)}{|\vec{q}(\tau)|^{3}}\right|+\left|Z_{\infty} \frac{\vec{q}(\tau)-\vec{r}(\tau)}{|\vec{q}(\tau)-\vec{r}(\tau)|^{3}}-Z_{\infty} \frac{\vec{q}(\tau)}{|\vec{q}(\tau)|^{3}}\right| \\
& \leq \frac{C_{1} R_{\min }}{|\vec{q}(\tau)|^{2+\epsilon}}+\frac{C_{1} R_{\min }}{\min (|\vec{q}(\tau)|,|\vec{q}(\tau)-\vec{r}(\tau)|)^{2+\epsilon}} \leq \frac{9 C_{1} R_{\min }}{|\vec{q}(\tau)|^{2+\epsilon}}
\end{aligned}
$$

by the decay assumptions (2.5) and (2.6) (which are valid for the asymptotic potential, too, see Remark 2.3.2), and Definition (6.9). For the last inequality we used $|\vec{r}(\tau)| \leq \frac{1}{2}|\vec{q}(\tau)|$, following from (2.14).

Estimating $|\vec{q}(\tau)|$ with (2.16) gives

$$
\begin{aligned}
& \left|\int_{s}^{\infty} d \tau\left(Z_{\infty} \frac{\vec{q}(\tau)-\vec{r}(\tau)}{|\vec{q}(\tau)-\vec{r}(\tau)|^{3}}-\nabla V(\vec{q}(\tau))\right)\right| \\
& \leq 9 C_{1} R_{\min } \int_{s}^{\infty}\left(\vec{q}_{0}^{2}+\frac{1}{2} E \tau^{2}\right)^{-\frac{1}{2}(2+\epsilon)} d \tau \\
& \leq \frac{9(2+\sqrt{2}) C_{1} R_{\min }}{\sqrt{E}} \max \left(\sqrt{E} s, q_{0}\right)^{-1-\epsilon} .
\end{aligned}
$$

Hence a second integration yields

$$
\|\mathcal{F} \vec{r}\| \leq 9(2+\sqrt{2}) C_{1} R_{\min } \frac{1+1 / \epsilon}{E q_{0}^{\epsilon}} \leq \frac{C_{2} R_{\min }^{\epsilon}}{E q_{0}^{\epsilon}},
$$


and thus for $\epsilon=1$ or $E>C_{2} / R_{\min }$ or $\left|\vec{q}_{0}\right| \geq R_{\text {vir }}(E)$ large $\mathcal{F}$ maps $\mathcal{C}_{E}$ into itself. Similarly, for $\vec{r}_{i} \in \mathcal{C}_{E}$

$$
\begin{aligned}
& \left\|\mathcal{F} \vec{r}_{1}-\mathcal{F} \vec{r}_{2}\right\| \\
& \leq C_{1} \int_{0}^{\infty} d s \int_{s}^{\infty} d \tau \frac{\left|\vec{r}_{1}(\tau)-\vec{r}_{2}(\tau)\right|}{\min \left(\left|\vec{q}(\tau)-\vec{r}_{1}(\tau)\right|,\left|\vec{q}(\tau)-\vec{r}_{2}(\tau)\right|\right)^{2+\epsilon}} \\
& \leq 8 C_{1}\left\|\vec{r}_{1}-\vec{r}_{2}\right\| \int_{0}^{\infty} d s \int_{s}^{\infty} d \tau|\vec{q}(\tau)|^{-2-\epsilon} \\
& \leq 8(2+\sqrt{2}) C_{1} \frac{1+1 / \epsilon}{E q_{0}^{\epsilon}}\left\|\vec{r}_{1}-\vec{r}_{2}\right\| \leq \frac{8}{9} \frac{C_{2} R_{\min }^{\epsilon-1}}{E q_{0}^{\epsilon}}\left\|\vec{r}_{1}-\vec{r}_{2}\right\| .
\end{aligned}
$$

By (2.14) for $\epsilon=1$ or $E>C_{2} / R_{\min }$ or $\left|\vec{q}_{0}\right| \geq R_{\text {vir }}(E)$ large the r.h.s. of (6.13) is bounded above by $\frac{8}{9}\left\|\vec{r}_{1}-\vec{r}_{2}\right\|$, so that the map $\mathcal{F}: \mathcal{C}_{E} \rightarrow \mathcal{C}_{E}$ is a contraction, and thus has a unique fixed point $\vec{r} \in \mathcal{C}_{E}$. The first estimate in (6.7) follows from (6.11) and the second from (6.12).

After having shown unique existence of $\left(\Omega^{ \pm}\right)^{-1}: s^{ \pm} \rightarrow P_{\infty,+}$, we show unique existence of the Møller transforms by the same method of integral equations, interchanging the roles of the two potentials. This is indeed possible with the same constants (see Remark 2.3.2).

The proof of the remaining statements is the same as in [Sim].

By energy conservation and (2.4) the modulus asymptotic momenta $\vec{p}^{ \pm}(x)$ equals $\sqrt{2 H(x)}$, which is non-zero by Definition 6.1 of $s^{ \pm}$. So the asymptotic directions

$$
\hat{p}^{ \pm}: s^{ \pm} \rightarrow S^{2} \quad, \quad \hat{p}^{ \pm}(x):=\frac{\vec{p}^{ \pm}(x)}{\sqrt{2 H(x)}}
$$

are well-defined.

Corollary 6.4. $1 . s^{ \pm}=\Omega^{ \pm}\left(P_{\infty,+}\right)$, that is, every positive energy orbit which is unbounded in positive (negative) time is positively (negatively) asymptotic to a Kepler hyperbola.

2. The motion $\Phi^{t}$ generated by the Hamiltonian function $H: P \rightarrow \mathbb{R}$ is asymptotically complete, that is, up to a subset of Liouville measure zero the phase space consists of bound states and scattering states:

$$
\lambda(P \backslash(b \cup s))=0 .
$$

Similarly for the Liouville measure $\lambda_{E}$ on the energy shell $\Sigma_{E}$

$$
\lambda_{E}\left(\Sigma_{E} \backslash\left(b_{E} \cup s_{E}\right)\right)=0 \quad(E>0) .
$$

\section{The scattering transformation}

$$
S:=\Omega_{*}^{+} \circ \Omega^{-}: D \rightarrow P_{\infty}
$$

with domain $D:=\Omega_{*}^{-}(s) \subset P_{\infty,+}$ and range $\Omega_{*}^{+}(s)$ is continuous and $\Phi_{\infty}^{t}$-invariant, i.e.

$$
S \circ \Phi_{\infty}^{t}=\Phi_{\infty}^{t} \circ S .
$$


Proof.

1. The equality of $s^{ \pm}$with $\Omega^{ \pm}\left(P_{\infty,+}\right)$ is a consequence of Theorem 6.3 , since $\Omega_{*}^{ \pm}=\left(\Omega^{ \pm}\right)^{-1}$ is defined on $s^{ \pm}$.

2. This is a consequence the fact that $\Phi^{t}\left(b^{+, k}\right) \subset b^{+, k}$ for $t \geq 0$ but $\lambda\left(\Phi^{t}\left(b^{+, k}\right)\right)=$ $\lambda\left(b^{+, k}\right)$, since $\Phi^{t}$ is canonical (see [Hu]).

3. Follows from Theorem 6.3.

Theorem 6.5. Let $V$ be a Coulombic potential whose partial derivatives decay at infinity according to

$$
\partial_{q}^{\beta}\left(V(\vec{q})+\frac{Z_{\infty}}{|\vec{q}|}\right) \stackrel{\vec{q} \rightarrow \infty}{=} \mathcal{O}\left(|\vec{q}|^{-|\beta|-1-\epsilon}\right) \quad\left(\beta \in \mathbb{N}_{0}^{3}\right)
$$

for some $0<\varepsilon \leq 1$. Then the Mфller transformations $\Omega^{ \pm}: P_{\infty,+} \rightarrow s^{ \pm}$are $C^{\infty}$ diffeomorphisms and canonical transformations.

If $\left(\vec{p}_{0}, \vec{q}_{0}\right) \equiv x_{0}$ with $q_{0}:=\left|\vec{q}_{0}\right| \geq R_{\mathrm{vir}}(E)$ and $\pm\left\langle\vec{q}_{0}, \vec{p}_{0}\right\rangle \geq 0$, then for multi-indices $\alpha, \beta \in \mathbb{N}_{0}^{3}$ combined in $\gamma:=(\alpha, \beta), \partial_{x_{0}}^{\gamma}:=\partial_{p_{0}}^{\alpha} \partial_{q_{0}}^{\beta}$

$$
\begin{gathered}
\partial_{x_{0}}^{\gamma}\left(\vec{p}^{ \pm}\left(x_{0}\right)-\vec{p}_{0}\right)=\mathcal{O}\left(q_{0}^{-|\beta|-1} E^{-\frac{1}{2}(|\alpha|+1)}\right), \\
\left.\partial_{x_{0}}^{\gamma}\left(\vec{L}^{ \pm}\left(x_{0}\right)-\vec{L}\left(x_{0}\right)\right)=\mathcal{O}\left(q_{0}^{-|\beta|-\epsilon} E^{-\frac{1}{2}(|\alpha|+1)}\right)\right),
\end{gathered}
$$

and for $\left(\vec{P}_{0}, \vec{Q}_{0}\right):=\Omega^{ \pm}\left(\vec{p}_{0}, \vec{q}_{0}\right)$ and $E>E_{\mathrm{th}}$

$$
\begin{aligned}
& \partial_{x_{0}}^{\gamma}\left(\vec{P}_{0}-\vec{p}_{0}\right)=\mathcal{O}\left(q_{0}^{-|\beta|-1-\varepsilon} E^{-\frac{1}{2}(|\alpha|+1)}\right), \\
& \partial_{x_{0}}^{\gamma}\left(\vec{Q}_{0}-\vec{q}_{0}\right)=\mathcal{O}\left(q_{0}^{-|\beta|-\varepsilon} E^{-|\alpha| / 2-1}\right) .
\end{aligned}
$$

Remarks 6.6. 1) The above decay condition (6.16) is met by purely Coulombic potentials (2.7) with $\epsilon=1$.

2) It is more natural to rescale the momentum by setting $\vec{v}:=\vec{p} / \sqrt{2 E}$ (and going to the rescaled angular momentum $\vec{L} / \sqrt{2 E}$ ). In terms of these variables all estimates in Theorem 6.5 show an energy dependence of the order $\mathcal{O}(1 / E)$.

Proof. Note that all estimates of the theorem for $\gamma=0$ coincide with the ones of Theorem 6.3.

1) We estimate the derivatives of

$$
\vec{q}\left(t, x_{0}\right)=\vec{q}_{0}+t \vec{p}_{0}-\int_{0}^{t} \int_{0}^{s} \nabla V\left(\vec{q}\left(\tau, x_{0}\right)\right) d \tau d s .
$$


We have for $g:=|\gamma| \geq 1$

$$
\begin{aligned}
\partial_{x_{0}}^{\gamma} \vec{q}\left(t, x_{0}\right)= & \partial_{x_{0}}^{\gamma}\left(\vec{q}_{0}+t \vec{p}_{0}\right)-\int_{0}^{t} \int_{0}^{s} \nabla \partial_{x_{0}}^{\gamma} V\left(\vec{q}\left(\tau, x_{0}\right)\right) d \tau d s \\
= & \partial_{x_{0}}^{\gamma}\left(\vec{q}_{0}+t \vec{p}_{0}\right)- \\
& \sum_{N=1}^{g} \sum_{\substack{\gamma^{(1)}+\ldots+\gamma^{(N)}=\gamma \\
\left|\gamma^{(i)}\right|>0}} \int_{0}^{t} \int_{0}^{s} D^{N} \nabla V\left(\vec{q}\left(\tau, x_{0}\right)\right) \\
& \times\left(\partial_{x_{0}}^{\gamma^{(1)}} \vec{q}\left(\tau, x_{0}\right), \ldots, \partial_{x_{0}}^{\gamma^{(N)}} \vec{q}\left(\tau, x_{0}\right)\right) d \tau d s
\end{aligned}
$$

or

$$
\begin{aligned}
(11+\mathcal{Q})\left(\partial_{x_{0}}^{\gamma} \vec{q}\right)(t)= & \partial_{x_{0}}^{\gamma}\left(\vec{q}_{0}+t \vec{p}_{0}\right)- \\
& \sum_{\substack{g=2 \\
\gamma^{(1)}+\ldots+\gamma^{(N)}=\gamma \\
\left|\gamma^{(i)}\right|>0}} \int_{0}^{t} \int_{0}^{s} D^{N} \nabla V\left(\vec{q}\left(\tau, x_{0}\right)\right) \\
& \left(\partial_{x_{0}}^{\gamma^{(1)}} \vec{q}\left(\tau, x_{0}\right), \ldots, \partial_{x_{0}}^{\gamma^{(N)}} \vec{q}\left(\tau, x_{0}\right)\right) d \tau d s
\end{aligned}
$$

with the linear operator $\mathcal{Q} \equiv \mathcal{Q}_{x_{0}}$ given by

$$
\mathcal{Q}(\vec{w})(t):=\int_{0}^{t} \int_{0}^{s} D \nabla V\left(\vec{q}\left(\tau, x_{0}\right)\right) \vec{w}(\tau) d \tau d s \quad(t \geq 0) .
$$

We note that on the r.h.s. of (6.21) only partial derivatives or order $\left|\gamma^{(i)}\right|<g$ appear, so that we can perform an induction in $g$, if we are able to invert the operator $\mathbb{1}+\mathcal{Q}$.

We assume $\vec{w} \in \hat{\mathcal{C}}$ with

$$
\hat{\mathcal{C}}:=\left\{\vec{w} \in C\left([0, \infty), \mathbb{R}^{3}\right)\left|\|\vec{w}\|_{\lambda}:=\sup _{t \geq 0}\right| \vec{w}(t) \mid /\langle t\rangle_{\lambda}<\infty\right\}
$$

for

$$
\langle t\rangle_{\lambda}:=\sqrt{1+(\lambda t)^{2}}
$$

(note that $\hat{\mathcal{C}}$ is independent of the choice of $\lambda>0$ ).

$\mathcal{Q}$ maps $\hat{\mathcal{C}}$ into itself, and we want to prove that for all positive energies the operator norm of $\mathcal{Q}$ is strictly smaller than one.

If we assume

$$
q_{0} \geq R_{\mathrm{vir}}(E) \quad \text { and } \quad\left\langle\vec{q}_{0}, \vec{p}_{0}\right\rangle \geq 0,
$$

then estimate (2.16) holds:

$$
|\vec{q}(t)| \geq q_{0} \cdot\langle t\rangle_{\lambda} \quad \text { for all } t \geq 0, \text { with } \quad \lambda:=\sqrt{E / 2} / q_{0} .
$$

(6.16) implies that

$$
\partial_{q}^{\beta} V(\vec{q})=\mathcal{O}\left(|\vec{q}|^{-|\beta|-1}\right) .
$$


So we find $c_{N}>0$ such that

$$
\left\|D^{N} \nabla V(\vec{q}(\tau))\right\| \leq c_{N}\left(q_{0}\langle\tau\rangle_{\lambda}\right)^{-N-2} \quad(N \in \mathbb{N}) .
$$

For estimating the norm of $\mathcal{Q}$, we may restrict ourselves to $\vec{w} \in \hat{\mathcal{C}}$ with

$$
\|\vec{w}\|_{\lambda}=1 .
$$

Inserting (6.27) and (6.28) into (6.22), we get

$$
\begin{aligned}
|\mathcal{Q}(\vec{w})(t)| & \leq c_{1} q_{0}^{-3} \int_{0}^{t} \int_{0}^{s}\langle\tau\rangle_{\lambda}^{-2} d \tau d s \leq \frac{c_{1}}{\sqrt{E / 2} q_{0}^{2}} \int_{0}^{t} \int_{0}^{\infty}\left(1+u^{2}\right)^{-1} d u d s \\
& =\frac{c_{1} \pi}{\sqrt{2 E} q_{0}^{2}} t \leq \frac{c_{1} \pi}{E q_{0}}\langle t\rangle_{\lambda} .
\end{aligned}
$$

Assuming (6.24), a suitable choice of the $E$-dependence of $R_{\mathrm{vir}}$ consistent with assumption (2.14) then gives

$$
\left\|\mathcal{Q}_{x_{0}}\right\|_{\lambda} \leq \frac{1}{2} \quad \text { and } \quad\left\|\mathcal{Q}_{x_{0}}\right\|_{\lambda}=\mathcal{O}\left(1 /\left(q_{0} H\left(x_{0}\right)\right)\right)
$$

for the operator norm $\left\|\mathcal{Q}_{x_{0}}\right\|_{\lambda}$ of $\mathcal{Q}$ w.r.t. the norm $\|\cdot\|_{\lambda}$ in (6.23).

We return to (6.21) and estimate its r.h.s. Using (6.25) and (6.27), we get

$$
\begin{gathered}
\quad\left|\int_{0}^{t} \int_{0}^{s} D^{N} \nabla V\left(\vec{q}\left(\tau, x_{0}\right)\right)\left(\partial_{x_{0}}^{\gamma^{(1)}} \vec{q}\left(\tau, x_{0}\right), \ldots, \partial_{x_{0}}^{\gamma^{(N)}} \vec{q}\left(\tau, x_{0}\right)\right) d \tau d s\right| \\
\leq c_{N} q_{0}^{-N-2} \int_{0}^{t} \int_{0}^{s}\langle\tau\rangle_{\lambda}^{-2} d \tau d s \cdot \prod_{i=1}^{N}\left\|\partial_{x_{0}}^{\gamma^{(i)}} \vec{q}\left(\cdot, x_{0}\right)\right\|_{\lambda} \leq \text { const. }\langle t\rangle_{\lambda} q_{0}^{-|\beta|} E^{-\frac{1}{2}|\alpha|-1},
\end{gathered}
$$

assuming

$$
\left\|\partial_{x_{0}}^{\gamma^{\prime}} \vec{q}\left(\cdot, x_{0}\right)\right\|_{\lambda}=\mathcal{O}\left(q_{0}^{-\left|\beta^{\prime}\right|+\delta_{\left|\gamma^{\prime}\right|, 1}} E^{-\frac{1}{2}\left|\alpha^{\prime}\right|-1+\delta_{\left|\gamma^{\prime}\right|, 1}}\right) \quad \text { for } \quad 0<\left|\gamma^{\prime}\right|<g .
$$

Inserting (6.29) into (6.21), we see that (6.31) holds for the start of the induction, i.e. $\left|\gamma^{\prime}\right|=1$. Also, (6.30) is consistent with (6.31), so that (6.31) holds for all multi-indices $\gamma^{\prime} \in \mathbb{N}_{0}^{6}$.

2) Now similar to (6.21), the derivatives of the momentum meet the recursion

$$
\begin{aligned}
& \partial_{x_{0}}^{\gamma}\left(\vec{p}\left(t, x_{0}\right)-\vec{p}_{0}\right)= \\
& -\sum_{\substack{N=1 \\
\gamma^{(1)}+\ldots+\gamma^{(N)} \\
\left|\gamma^{(i)}\right|>0}}^{g} \int_{0}^{t} D^{N} \nabla V\left(\vec{q}\left(\tau, x_{0}\right)\right)\left(\partial_{x_{0}}^{\gamma^{(1)}} \vec{q}\left(\tau, x_{0}\right), \ldots, \partial_{x_{0}}^{\gamma^{(N)}} \vec{q}\left(\tau, x_{0}\right)\right) d \tau .
\end{aligned}
$$

We insert (6.27) and (6.31) into (6.32) and performing the time $t \rightarrow \infty$ limit. Then by Lebesgue's Dominated Convergence Theorem $\partial_{x_{0}}^{\gamma} \vec{p}^{+}\left(x_{0}\right)$ exists, and estimate (6.17) is valid. $\partial_{x_{0}}^{\gamma} \vec{p}^{+}$As a locally uniform limit of continuous functions, $\partial_{x_{0}}^{\gamma} \vec{p}^{+}$is continuous. 
3) Similar to the proof of (6.3) we use the ansatz

$$
\begin{aligned}
& \partial_{x_{0}}^{\gamma}\left(\vec{L}\left(x\left(t, x_{0}\right)\right)-\vec{L}\left(x_{0}\right)\right)=\int_{0}^{t} \partial_{x_{0}}^{\gamma} \frac{d}{d \tau} \vec{L}\left(x\left(\tau, x_{0}\right)\right) d \tau \\
& =\int_{0}^{t} \partial_{x_{0}}^{\gamma}\left(\nabla V_{\mathrm{sr}}\left(\vec{q}\left(\tau, x_{0}\right)\right) \times \vec{q}\left(\tau, x_{0}\right)\right) d \tau \\
& =\sum_{N=1}^{g} \sum_{\substack{\gamma^{(1)}+\ldots+\gamma^{(N)}=\gamma \\
\left|\gamma^{(i)}\right|>0}} \int_{0}^{t} D^{N}\left(\nabla V_{\mathrm{sr}}\left(\vec{q}\left(\tau, x_{0}\right)\right) \times \vec{q}\left(\tau, x_{0}\right)\right) \\
& \left(\partial_{x_{0}}^{\gamma^{(1)}} \vec{q}\left(\tau, x_{0}\right), \ldots, \partial_{x_{0}}^{\gamma^{(N)}} \vec{q}\left(\tau, x_{0}\right)\right) d \tau
\end{aligned}
$$

in order to estimate the smoothness of the difference between the actual and the asymptotic angular momentum.

Note that the short range potential $V_{\mathrm{sr}}=V-V_{\infty}$ appears in assumption (6.16). Thus for all $t \geq 0$ the above expression is bounded above by

$$
c_{N} q_{0}^{-|\beta|-1-\varepsilon} E^{-\frac{1}{2}|\alpha|} \int_{0}^{\infty}\langle\tau\rangle_{\lambda}^{-1-\varepsilon} d \tau \leq \text { const. } q_{0}^{-|\beta|-\varepsilon} E^{-(|\alpha|+1) / 2} .
$$

So by dominated convergence $\partial_{x_{0}}^{\gamma} \vec{L}^{+}\left(x_{0}\right)$ exists, and is estimated by (6.18).

4) As in Theorem 6.3 we now consider the Kepler hyperbola

$$
\left(\vec{P}\left(t ; x_{0}\right), \vec{Q}\left(t ; x_{0}\right)\right)=\Phi_{\infty}^{t}\left(X_{0}\right) \quad, \quad X_{0} \equiv\left(\vec{P}_{0}, \vec{Q}_{0}\right)=\Omega^{+}\left(x_{0}\right)
$$

which is positive asymptotic to $\Phi^{t}\left(x_{0}\right)=\left(\vec{p}\left(t, x_{0}\right), \vec{q}\left(t, x_{0}\right)\right)$ and write

$$
\vec{r}(t) \equiv \vec{r}\left(t ; x_{0}\right):=\vec{q}\left(t, x_{0}\right)-\vec{Q}\left(t ; x_{0}\right) \text {. }
$$

Then $\vec{r}$ is the solution of the integral equation (6.10). Thus formally

$$
\begin{aligned}
\partial_{x_{0}}^{\gamma} \vec{r}(t) & =\int_{t}^{\infty} \int_{s}^{\infty} \partial_{x_{0}}^{\gamma} \nabla\left(V_{\infty}\left(\vec{Q}\left(\tau ; x_{0}\right)\right)-V\left(\vec{q}\left(\tau, x_{0}\right)\right)\right) d \tau d s \\
& =I_{1}^{\gamma}\left(t ; x_{0}\right)+I_{2}^{\gamma}\left(t ; x_{0}\right)
\end{aligned}
$$

with the integrals

$$
I_{1}^{\gamma}\left(t ; x_{0}\right):=-\int_{t}^{\infty} \int_{s}^{\infty} \partial_{x_{0}}^{\gamma} \nabla V_{\mathrm{sr}}\left(\vec{q}\left(\tau, x_{0}\right)\right) d \tau d s
$$

and

$$
I_{2}^{\gamma}\left(t ; x_{0}\right):=\int_{t}^{\infty} \int_{s}^{\infty} \partial_{x_{0}}^{\gamma} \nabla\left(V_{\infty}\left(\vec{Q}\left(\tau ; x_{0}\right)\right)-V_{\infty}\left(\vec{q}\left(\tau, x_{0}\right)\right)\right) d \tau d s .
$$


We estimate the first integral in a similar fashion as the r.h.s. of (6.20), but use the stronger estimate (6.16) for $V_{\mathrm{sr}}$ instead of (6.26) for $V$. We thus obtain (with $g=|\gamma|)$

$$
\begin{aligned}
& \left|I_{1}^{\gamma}\left(t ; x_{0}\right)\right| \leq \sum_{N=1}^{g} \sum_{\substack{\gamma^{(1)}+\ldots+\gamma^{(N)}=\gamma \\
\left|\gamma^{(i)}\right|>0}} \\
& \quad \int_{t}^{\infty} \int_{s}^{\infty}\left|D^{N} \nabla V_{\mathrm{sr}}\left(\vec{q}\left(\tau, x_{0}\right)\right)\left(\partial_{x_{0}}^{\gamma^{(1)}} \vec{q}\left(\tau, x_{0}\right), \ldots, \partial_{x_{0}}^{\gamma^{(N)}} \vec{q}\left(\tau, x_{0}\right)\right)\right| d \tau d s \\
& \leq \text { const. } q_{0}^{-N-2-\varepsilon} \int_{t}^{\infty} \int_{s}^{\infty}\langle\tau\rangle_{\lambda}^{-2-\varepsilon} d \tau d s \cdot \prod_{i=1}^{N}\left\|\partial_{x_{0}}^{\gamma^{(i)}} \vec{q}\left(\cdot, x_{0}\right)\right\|_{\lambda} \\
& \leq \text { const. } q_{0}^{-|\beta|-\varepsilon} E^{-\frac{1}{2}|\alpha|-1} .
\end{aligned}
$$

Unlike $I_{1}^{\gamma}, I_{2}^{\gamma}$ depends on $\vec{r}$. So we split it into

$$
I_{2}^{\gamma}=\mathcal{P}\left(\partial_{x_{0}}^{\gamma} \vec{r}\right)+I_{3}^{\gamma}+I_{4}^{\gamma}
$$

with the linear operator

$$
\begin{array}{r}
\mathcal{P}(\vec{w})(t):=-\int_{t}^{\infty} \int_{s}^{\infty} D \nabla V_{\infty}\left(\vec{Q}\left(\tau ; x_{0}\right)\right) \vec{w}(\tau) d \tau d s \quad(t \geq 0) \\
I_{3}^{\gamma}:=\sum_{\substack { N=1 \\
\begin{subarray}{c}{\gamma^{(1)}+\ldots+\gamma^{(N)}=\gamma \\
\left|\gamma^{(i)}\right|>0{ N = 1 \\
\begin{subarray} { c } { \gamma ^ { ( 1 ) } + \ldots + \gamma ^ { ( N ) } = \gamma \\
| \gamma ^ { ( i ) } | > 0 } }\end{subarray}} \int_{t}^{\infty} \int_{s}^{\infty} D^{N} \nabla\left[V_{\infty}\left(\vec{Q}\left(\tau ; x_{0}\right)\right)-V_{\infty}\left(\vec{q}\left(\tau, x_{0}\right)\right)\right] \\
\times\left(\partial_{x_{0}}^{\gamma^{(1)}} \vec{q}\left(\tau, x_{0}\right), \ldots, \partial_{x_{0}}^{\gamma^{(N)}} \vec{q}\left(\tau, x_{0}\right)\right) d \tau d s
\end{array}
$$

and

$$
\begin{aligned}
I_{4}^{\gamma}:=\sum_{\substack{N=2 \\
\gamma^{(1)}+\ldots+\gamma^{(N)}=\gamma \\
\left|\gamma^{(i)}\right|>0}}^{g} \sum_{t}^{\infty} \int_{s}^{\infty}\left[D^{N} \nabla V_{\infty}\left(\vec{Q}\left(\tau ; x_{0}\right)\right)\left(\partial_{x_{0}}^{\gamma^{(1)}} \vec{Q}\left(\tau ; x_{0}\right), \ldots, \partial_{x_{0}}^{\gamma^{(N)}} \vec{Q}\left(\tau ; x_{0}\right)\right)\right. \\
\left.\quad-D^{N} \nabla V_{\infty}\left(\vec{Q}\left(\tau ; x_{0}\right)\right)\left(\partial_{x_{0}}^{\gamma^{(1)}} \vec{q}\left(\tau, x_{0}\right), \ldots, \partial_{x_{0}}^{\gamma^{(N)}} \vec{q}\left(\tau, x_{0}\right)\right)\right] d \tau d s .
\end{aligned}
$$

On the space of bounded $\vec{w}, \mathcal{P} \equiv \mathcal{P}_{x_{0}}$ is bounded, since for $\sup _{t}|\vec{w}(t)|=1$

$$
\begin{aligned}
|\mathcal{P}(\vec{w})(t)| & \leq\left|Z_{\infty}\right| q_{0}^{-3} \int_{0}^{\infty} \int_{s}^{\infty}\langle\tau\rangle_{\lambda}^{-3} d \tau d s \\
& =\frac{2\left|Z_{\infty}\right|}{q_{0} E} \int_{0}^{\infty}\left(1-\frac{u}{\sqrt{1+u^{2}}}\right) d u=\frac{2\left|Z_{\infty}\right|}{q_{0} E} .
\end{aligned}
$$


Assuming (6.24), a suitable choice of the $E$-dependence of $R_{\text {vir }}$ consistent with assumption (2.14) thus gives

$$
\left\|\mathcal{P}_{x_{0}}\right\| \leq \frac{1}{2} \quad \text { and } \quad\left\|\mathcal{P}_{x_{0}}\right\|=\mathcal{O}\left(1 /\left(q_{0} H\left(x_{0}\right)\right)\right),
$$

so that we can invert $11-\mathcal{P}$ for all energies $E>0$.

$I_{3}^{\gamma}$ and $I_{4}^{\gamma}$ only contain derivatives $\partial_{x_{0}}^{\gamma^{\prime}} \vec{r}\left(\tau ; x_{0}\right)$ with $\left|\gamma^{\prime}\right|<g$.

Majorizing $V_{\infty}(\vec{Q})-V_{\infty}(\vec{q})$ by $2\left|D V_{\infty}(\vec{q}) \vec{r}\right|$, the terms in $I_{3}^{\gamma}$ are estimated by

$$
\begin{aligned}
\text { const. } q_{0}^{-N-3} \int_{0}^{\infty} \int_{s}^{\infty}\langle\tau\rangle_{\lambda}^{-3}\left|\vec{r}\left(\tau ; x_{0}\right)\right| d \tau d s \prod_{i=1}^{N}\left\|\partial_{x_{0}}^{\gamma^{(i)}} \vec{q}\left(\cdot, x_{0}\right)\right\|_{\lambda}= \\
\mathcal{O}\left(q_{0}^{-|\beta|-1} E^{-\frac{1}{2}|\alpha|-1}\right),
\end{aligned}
$$

since $\left|\vec{r}\left(t, x_{0}\right)\right|<R_{\min }$ (see (6.9)), and using (6.31).

Finally, inserting $\vec{Q}=\vec{q}-\vec{r}$ into the first $N$-linear form of $I_{4}^{\gamma}$ and expanding, we see that each term contains at least one factor of the form $\partial_{x_{0}}^{\gamma^{(i)}} \vec{r}\left(\cdot, x_{0}\right)$. So

$$
I_{4}^{\gamma}=\mathcal{O}\left(q_{0}^{-|\beta|-1} E^{-\frac{1}{2}|\alpha|-1}\right)
$$

too.

Together with the above estimates for $I_{1}^{\gamma}$ and $I_{3}^{\gamma}$ this finally shows that

$$
\partial_{x_{0}}^{\gamma} \vec{r}\left(\tau ; x_{0}\right)=(11-\mathcal{P})^{-1}\left(I_{1}^{\gamma}+I_{3}^{\gamma}+I_{4}^{\gamma}\right)=\mathcal{O}\left(q_{0}^{-|\beta|-\varepsilon} E^{-\frac{1}{2}|\alpha|-1}\right),
$$

which is equivalent to the second assertion in (6.19). By a parametrized version of the Banach Fixed Point Theorem (see, e.g. [DG], Proposition A.2.2), $x_{0} \mapsto$ $\partial_{x_{0}}^{\gamma} \vec{r}\left(\cdot ; x_{0}\right)$ is continuous.

The first assertion in (6.19) follows similarly, since

$$
\partial_{x_{0}}^{\gamma}\left(\vec{P}_{0}-\vec{p}_{0}\right)=-\int_{t}^{\infty} \partial_{x_{0}}^{\gamma} \nabla\left(V_{\infty}\left(\vec{Q}\left(\tau ; x_{0}\right)\right)-V\left(\vec{q}\left(\tau, x_{0}\right)\right)\right) d \tau .
$$

\section{The flow between near-collisions}

We remind the reader of the relation (2.13) for the virial radius $R_{\mathrm{vir}}(E)$. We assume that $E_{\text {th }}>V_{\text {max }}$ so that we may assume $R_{\text {vir }}(E) \equiv R_{\text {vir }}$ for all $E>E_{\text {th }}$.

By the virial identity (2.12) a trajectory of energy $E$ leaving the interaction zone $\mathcal{I Z}=\left\{\vec{q} \in \mathbb{R}_{\vec{q}}^{3}|| \vec{q} \mid \leq R_{\text {vir }}\left(E_{\text {th }}\right)\right\}$ cannot reenter it.

We show first that a particle cannot stay inside the interaction zone for a long time without having close encounters with the nuclei. Then we will control long trajectories within $\mathcal{I Z}$ by Poincaré section techniques.

To quantify this, let

$$
\mathcal{I Z}(r):=\mathcal{I Z} \backslash \bigcup_{l=1}^{n} \operatorname{int}\left(B_{l}(r)\right)
$$


$B_{l}(r)=\left\{\vec{q} \in \mathbb{R}_{\vec{q}}^{3}|| \vec{q}-\vec{s}_{l} \mid \leq r\right\}$ being the ball of radius $r$ around the $l$ th nucleus.

Instead of considering the restriction $\left.\Phi^{t}\right|_{\Sigma_{E}}$ of the flow generated by $H$, it is technically convenient to consider the flow $\Phi_{E}^{t}$ generated by

$$
\hat{H}_{E}(\vec{v}, \vec{x}):=\frac{1}{2}|\vec{v}|^{2}+\frac{V(\vec{x})}{2 E} \quad \text { on } \quad\left(\hat{H}_{E}\right)^{-1}\left(\frac{1}{2}\right) .
$$

We then have for $(\vec{p}(t), \vec{q}(t)) \equiv \Phi^{t}\left(\vec{p}_{0}, \vec{q}_{0}\right)$ and $(\vec{v}(s), \vec{x}(s)) \equiv \Phi_{E}^{s}\left(\vec{v}_{0}, \vec{x}_{0}\right)$ with

$$
\left(\vec{v}_{0}, \vec{x}_{0}\right):=\left(\vec{p}_{0} / \sqrt{2 E}, \vec{q}_{0}\right)
$$

$$
(\vec{p}(t), \vec{q}(t))=(\sqrt{2 E} \vec{v}(\sqrt{2 E} t), \vec{x}(\sqrt{2 E} t)) .
$$

In the lemma below we use the standard Euclidean metric on $\mathbb{R}_{\vec{v}}^{3} \times \mathbb{R}_{\vec{x}}^{3}$.

Lemma 7.1. For $E>E_{\text {th }}$ the rescaled flow $\Phi_{E}^{t}$ in $\mathcal{I Z}\left(c_{q}\right)$ is $C^{1}$-near to the linear flow generated by the Hamiltonian function $\hat{H}_{\infty} \equiv \frac{1}{2}|\vec{v}|^{2}$ in the sense that

$$
\begin{aligned}
& \sup _{s \in[0, T]}\left(\left|\Phi_{E}^{s}\left(\vec{v}_{0}, \vec{x}_{0}\right)-\left(\vec{v}_{0}, \vec{x}_{0}+s \vec{v}_{0}\right)\right|+\left\|D \Phi_{E}^{s}\left(\vec{v}_{0}, \vec{x}_{0}\right)-\left(\begin{array}{cc}
\mathbb{1} & 0 \\
s \| & \mathbb{1}
\end{array}\right)\right\|\right) \\
& \quad=\mathcal{O}(1 / E)
\end{aligned}
$$

if

$$
x([0, T]) \subset \mathcal{I Z}\left(c_{q}\right)
$$

Moreover, condition (7.3) is never satisfied if $T \geq 3 R_{\mathrm{vir}}$.

Proof. We assume that $T \leq 3 R_{\mathrm{vir}}$, prove estimate (7.2) and then the necessity of that assumption. The constants

$$
L_{0}:=\sup _{\vec{q} \in \mathcal{I Z}\left(c_{q}\right)}|V(\vec{q})| \quad, \quad L_{1}:=\sup _{\vec{q} \in \mathcal{I} \mathcal{Z}\left(c_{q}\right)}|\nabla V(\vec{q})|
$$

and

$$
L_{2}:=\sup _{\vec{q} \in \mathcal{I} \mathcal{Z}\left(c_{q}\right)}\left|D^{2} V(\vec{q})\right|
$$

are finite, since $\mathcal{I Z}\left(c_{q}\right)$ is compact. We bound the differences

$$
Z_{1}(s):=\left(\vec{v}(s)-\vec{v}_{0}, \vec{x}(s)-\left(\vec{x}_{0}+s \vec{v}_{0}\right)\right)
$$

and

$$
Z_{2}(s):=D \Phi_{E}^{s}\left(\vec{v}_{0}, \vec{x}_{0}\right)-\left(\begin{array}{cc}
\mathbb{1} & 0 \\
s \| & \mathbb{1}
\end{array}\right)
$$

in (7.2) using their integral equations

$$
\vec{x}(s)-\left(\vec{x}_{0}+s \vec{v}_{0}\right)=-(2 E)^{-1} \int_{0}^{s} \int_{0}^{u} \nabla V(\vec{x}(\tau)) d \tau d u
$$


and

$$
Z_{2}(s)=-(2 E)^{-1} \int_{0}^{s}\left(\begin{array}{cc}
0 & D^{2} V(\vec{x}(\tau)) \\
0 & 0
\end{array}\right) d \tau
$$

and thus obtain

$$
\sup _{s \in[0, T]}\left|Z_{1}(s)\right| \leq L_{1} \frac{T+T^{2} / 2}{2 E} \quad, \quad \sup _{s \in[0, T]}\left|Z_{2}(s)\right| \leq L_{2} \frac{T}{2 E} .
$$

This is indeed of order $\mathcal{O}(1 / E)$.

Moreover, for $E>E_{\mathrm{th}} \geq \max \left(4 L_{0}, L_{1} R_{\mathrm{vir}}\right)$ we have

$$
\left|\vec{v}_{0}\right|=\sqrt{1-V\left(\vec{x}_{0}\right) / E} \geq \frac{\sqrt{3}}{2}
$$

and $\left|\vec{x}(s)-\left(\vec{x}_{0}+s \vec{v}_{0}\right)\right| \leq R_{\mathrm{vir}} / 4$ for $s \leq T \leq 3 R_{\mathrm{vir}}$ so that for $T:=3 R_{\mathrm{vir}}$

$$
\left|\vec{x}(T)-\vec{x}_{0}\right| \geq\left|\vec{v}_{0}\right| T-R_{\mathrm{vir}} / 4 \geq\left(\frac{3 \sqrt{3}}{2}-\frac{1}{4}\right) R_{\mathrm{vir}}>2 R_{\mathrm{vir}} .
$$

Thus for $T=3 R_{\text {vir }}$ we obtain a contradiction with our assumption (7.3), since the diameter of $\mathcal{I Z}$ equals $2 R_{\text {vir }}$ so that $\vec{x}_{0}$ and $\vec{x}(T)$ cannot be both $\in \mathcal{I Z}$.

\section{The single scattering process}

We now consider motion inside the ball $B_{l}\left(c_{q}\right)$ near the $l$ th singularity, using the KS estimates of Proposition 4.1. For initial conditions in the phase space region

$$
\mathcal{D}_{l}:=\pi\left(\mathcal{D}_{l}\right)=\left\{x \in P|| \vec{q}(x)-\vec{s}_{l} \mid \leq c_{q}, H(x)>E_{\mathrm{th}}\right\}
$$

over the ball the exit times

$$
T_{l}^{ \pm}: \mathcal{D}_{l} \rightarrow \mathbb{R} \quad, \quad T_{l}^{ \pm}\left(x_{0}\right):= \pm \inf \left\{t \geq 0|| \vec{q}\left( \pm t, x_{0}\right)-\vec{s}_{l} \mid=c_{q}\right\}
$$

are well-defined and smooth. We compare them with the exit times $T_{L, l}^{ \pm}$for the purely Keplerian motion in $B_{l}\left(c_{q}\right)$ generated by (5.4), and set

$$
\Psi_{l}^{ \pm}: \mathcal{D}_{l} \rightarrow \partial \mathcal{D}_{l}, \quad x \mapsto \Phi\left(T_{l}^{ \pm}(x), x\right)
$$

and $\Psi_{L, l}^{ \pm}(x):=\Phi_{L}\left(T_{L, l}^{ \pm}(x), x\right)$.

Similar to (4.16) we define the pericentric hypersurface

$$
\begin{aligned}
\mathcal{H}_{l} & :=\pi\left(\mathcal{H}_{l}\right) \\
& =\left\{x \in \mathcal{D}_{l} \mid \vec{q}(x)=\vec{s}_{l} \quad \text { or } \quad\left\langle\vec{p}(x), \vec{q}(x)-\vec{s}_{l}\right\rangle=0\right\}
\end{aligned}
$$

on which the pericentric time $T_{l}^{0}: \mathcal{D}_{l} \rightarrow \mathbb{R}$ vanishes. If $Z_{l}>0$ we smoothly extend $T_{l}^{0}$ by demanding that

$$
\Psi_{l}^{0}(x):=\Phi\left(T_{l}^{0}(x), x\right) \in \mathcal{H}_{l}, \quad\left(x \in \mathcal{D}_{l}\right) .
$$


In analogy to (4.13) we introduce the diffeomorphism

$$
\Psi_{l}: \partial \mathcal{D}_{l} \rightarrow \partial \mathcal{D}_{l}, \quad x \equiv(\vec{p}, \vec{q}) \mapsto\left\{\begin{array}{l}
\Psi_{l}^{+}(x),\left\langle\vec{p}, \vec{q}-\vec{s}_{l}\right\rangle \leq 0 \\
\Psi_{l}^{-}(x),\left\langle\vec{p}, \vec{q}-\vec{s}_{l}\right\rangle>0
\end{array}\right.
$$

that permutes incoming and outgoing data and its analog $\Psi_{L, l}$ for the Kepler flow.

We first consider the Kepler flow (with potential $\vec{q} \mapsto-Z_{l} /\left|\vec{q}-\vec{s}_{l}\right|$ ) with incoming, resp. outgoing coordinates

$$
\left(\begin{array}{l}
\vec{p}^{-} \\
\vec{q}^{-}
\end{array}\right) \in \partial \mathcal{D}_{l} \cap \Sigma_{E} \quad \text { and } \quad\left(\begin{array}{l}
\vec{p}^{+} \\
\vec{q}^{+}
\end{array}\right):=\Psi_{L, l}\left(\begin{array}{l}
\vec{p}^{-} \\
\vec{q}^{-}
\end{array}\right) .
$$

It is more convenient to work with the coordinates

$$
\vec{v}^{ \pm}:=\frac{\vec{p}^{ \pm}}{\sqrt{2 E}} \quad \text { and } \quad \vec{w}^{ \pm}:=\frac{\vec{q}^{ \pm}-\vec{s}_{l}}{c_{q}}
$$

Lemma 8.1. The Kepler transformation $\Psi_{L, l}$ is given by

$$
\left\{\begin{aligned}
\vec{v}^{+}\left(\vec{v}^{-}, \vec{w}^{-}\right) & =\frac{\left(1-u^{2}(1+\beta)\right) \vec{v}^{-}-u \beta \vec{w}^{-}}{1-u^{2}} \\
\vec{w}^{+}\left(\vec{v}^{-}, \vec{w}^{-}\right) & =\frac{2 u\left(1-u^{2}(1+\beta / 2)\right) \vec{v}^{-}+\left(1-u^{2}(1+\beta)\right) \vec{w}^{-}}{1-u^{2}}
\end{aligned}\right.
$$

where

$$
\beta:=\frac{Z_{l}}{c_{q} E} \quad \text { and } \quad u \equiv u\left(\vec{v}^{-}, \vec{w}^{-}\right):=-\frac{\left\langle\vec{v}^{-}, \vec{w}^{-}\right\rangle}{1+\frac{1}{2} \beta}=-u\left(\vec{v}^{+}, \vec{w}^{+}\right) .
$$

Proof. For simplicity of notation we assume $\vec{s}_{l}=\overrightarrow{0}$. Since the KS transformation (3.2) transforms the scalar product in the formula (4.15)

$$
u=\frac{2\langle\tilde{P}, Q\rangle}{|\tilde{P}|^{2}+|Q|^{2}}
$$

into

$$
\langle\tilde{P}, Q\rangle=\frac{\operatorname{tr}\left(P Q^{*}\right)}{4 \sqrt{2 E}}=-\frac{\operatorname{tr}\left(p q^{*}\right)}{2 \sqrt{2 E}}=-\frac{\langle\vec{p}, \vec{q}\rangle}{\sqrt{2 E}}
$$

$|\tilde{P}|^{2}=c_{q}\left|\vec{v}^{-}\right|^{2}=c_{q}(1+\beta)$ and $|Q|^{2}=c_{q}$, we see that the definition (8.5) of $u$ is consistent with the definition in (4.15).

Eqs. (8.4) are obtained by inserting (4.15) into the $\mathrm{KS}$ formula (3.2) for $\left(\vec{p}^{+}, \vec{q}^{+}\right)$, noticing that

$$
-\tilde{P}^{*} I_{3} \tilde{P}=\frac{p q p}{2 E}=\frac{|\vec{p}|^{2} \vec{q}-2\langle\vec{p}, \vec{q}\rangle \vec{p}}{2 E}
$$

follows from the three-term-identity

$$
Y X Y=2\left\langle X^{*}, Y\right\rangle Y-\langle Y, Y\rangle X^{*} \quad(X, Y \in \mathbb{H})
$$

of quaternions, applied to $\vec{p}, \vec{q} \in \operatorname{Im} \mathbb{H}$. 
As we assumed that the NC-condition holds (no three singularities on a line), bounded orbits must be scattered by an angle $\varangle\left(\vec{v}^{+}, \vec{v}^{-}\right) \leq \vartheta$ which must be at least of the order of $\alpha_{\min }>0$ (see (2.10)). This can only happen if the mismatch between the initial velocity $\vec{v}^{-}$and position $\vec{w}^{-}$is only of the order $\mathcal{O}(1 / E)$. We first show this for Kepler scattering:

Lemma 8.2. For all energies $E>E_{\mathrm{th}} \geq 4 Z_{\max } / c_{q}$, angles $\theta \in\left[\pi \cdot E_{\mathrm{th}} / E, \pi\right]$, and initial conditions $\left(\vec{p}^{-}, \vec{q}^{-}\right) \in \partial \mathcal{D}_{l} \cap \Sigma_{E}$ and $u \geq 0$ with

$$
\left|\frac{\vec{v}^{-}}{\left|\vec{v}^{-}\right|}+\vec{w}^{-}\right| \geq \frac{\pi \cdot E_{\mathrm{th}}}{\theta \cdot E},
$$

Kepler scattering is in the forward direction, namely the total scattering angle is bounded by $\varangle\left(\vec{v}^{+}, \vec{v}^{-}\right) \leq \theta$.

Proof. Since the parameter $u \geq 0$ from (8.5) equals

$$
u\left(\vec{v}^{-}, \vec{w}^{-}\right)=-\frac{\left\langle\vec{v}^{-} /\left|\vec{v}^{-}\right|, \vec{w}^{-}\right\rangle \sqrt{1+\beta}}{1+\frac{1}{2} \beta} \leq-\left\langle\frac{\vec{v}^{-}}{\left|\vec{v}^{-}\right|}, \vec{w}^{-}\right\rangle
$$

so that

$$
1-u^{2}=(1+u)(1-u) \geq 1-u \geq \frac{1}{2}\left|\frac{\vec{v}^{-}}{\left|\vec{v}^{-}\right|}+\vec{w}^{-}\right|^{2},
$$

we get from formula (8.4) for $\vec{v}^{+}$that

$$
\begin{aligned}
\left|\vec{v}^{+}-\vec{v}^{-}\right| & =\frac{u|\beta|}{1-u^{2}}\left|u \vec{v}^{-}+\vec{w}^{-}\right| \\
& \leq \frac{u|\beta|}{1-u^{2}}\left(\left|\frac{\vec{v}^{-}}{\left|\vec{v}^{-}\right|}+\vec{w}^{-}\right|+\left|\vec{v}^{-}\right|\left((1-u)+|1-1 /| \vec{v}^{-}||\right)\right)
\end{aligned}
$$

with $\beta$ from (8.5).

For $E>E_{\text {th }}$ we have $|\beta|<\left|Z_{l}\right| /\left(c_{q} E_{\text {th }}\right) \leq \frac{1}{4}$, so that $\left|\vec{v}^{-}\right|=\sqrt{1+\beta} \in\left[\frac{1}{2}, 2\right]$. Thus inserting the last two estimates of (8.7) and (8.6) gives

$$
\begin{aligned}
\left|\vec{v}^{+}-\vec{v}^{-}\right| & \leq 2|\beta|\left(\frac{\theta \cdot E}{\pi \cdot E_{\mathrm{th}}}+1+\frac{|1-1 /| \vec{v}^{-}||}{1-u^{2}}\right) \\
& =\frac{2\left|Z_{l}\right|}{c_{q}}\left(\frac{\theta}{\pi \cdot E_{\mathrm{th}}}+E^{-1}+E^{-1} \frac{\left|1-\frac{1}{\sqrt{1+\beta}}\right|}{1-u^{2}}\right) \\
& \leq \frac{2\left|Z_{l}\right|}{c_{q}}\left(\frac{\theta}{\pi \cdot E_{\mathrm{th}}}+\frac{\theta}{\pi \cdot E_{\mathrm{th}}}+\frac{2\left|Z_{l}\right|}{c_{q}} \frac{\theta^{2}}{\pi^{2} \cdot E_{\mathrm{th}}^{2}}\right) \\
& \leq \frac{\theta}{2 \pi}+\frac{\theta}{2 \pi}+\frac{\theta^{2}}{(2 \pi)^{2}} \leq \frac{5}{4 \pi} \theta
\end{aligned}
$$

This proves the assertion, since

$$
\varangle\left(\vec{v}^{+}, \vec{v}^{-}\right) \leq \frac{\pi}{2} \frac{\left|\vec{v}^{+}-\vec{v}^{-}\right|}{\left|\vec{v}^{-}\right|} \leq \frac{5}{8}(1+\beta)^{-1 / 2} \theta \leq \theta .
$$


Later on we will study the linearization of the flow $\Phi^{t}$, using Poincaré section techniques. Therefore we now calculate the linearization of the Kepler transformation (8.4), for tangent vectors $\left(\delta \vec{p}^{-}, \delta \vec{q}^{-}\right)$in the four-dimensional subspace

$$
T_{\left(\vec{p}^{-}, \vec{q}^{-}\right)}\left(\partial \mathcal{D}_{l} \cap \Sigma_{E}\right),
$$

that is, for variations $\left(\delta \vec{v}^{-}, \delta \vec{w}^{-}\right)$of (8.3) meeting

$$
\left\langle\vec{v}^{-}, \delta \vec{v}^{-}\right\rangle=0 \quad, \quad\left\langle\vec{w}^{-}, \delta \vec{w}^{-}\right\rangle=0
$$

Lemma 8.3. The linearization of the Kepler Transformation (8.4) is given by

$\delta \vec{v}^{+}=\frac{\left(1-u^{2}\right)\left[\left(\left(1-u^{2}\right)-u^{2} \beta\right) \delta \vec{v}^{-}-u \beta \delta \vec{w}^{-}\right]-\beta\left[2 u \vec{v}^{-}+\left(1+u^{2}\right) \vec{w}^{-}\right] d u}{\left(1-u^{2}\right)^{2}}$ and

$$
\begin{aligned}
\delta \vec{w}^{+}= & \frac{\left(2 u\left(1-u^{2}\right)-u^{3} \beta\right) \delta \vec{v}^{-}+\left(\left(1-u^{2}\right)-u^{2} \beta\right) \delta \vec{w}^{-}}{1-u^{2}} \\
& +\frac{\left[\left(2\left(1-u^{2}\right)^{2}+\beta u^{2}\left(u^{2}-3\right)\right) \vec{v}^{-}-2 u \beta \vec{w}^{-}\right] d u}{\left(1-u^{2}\right)^{2}}
\end{aligned}
$$

with

$$
d u:=-\frac{\left\langle\vec{w}^{-}, \delta \vec{v}^{-}\right\rangle+\left\langle\vec{v}^{-}, \delta \vec{w}^{-}\right\rangle}{1+\frac{1}{2} \beta} .
$$

Proof. By differentiation of (8.4).

As the next lemma shows, there are two regimes of Kepler scattering:

- hard scattering: If the scattering angle is larger than $c_{1} / \sqrt{E}$, then linearized scattering is basically a reflection combined with scaling. In particular, for an energy-independent scattering angle the Liapunov exponent is of the approximate size $E$.

- soft scattering: If, however, the scattering angle $\Delta \psi$ is smaller than $c_{2} / \sqrt{E}$, then linearized scattering is a perturbation of free motion.

Lemma 8.4. 1) For $c_{1}>0$ and $0 \leq \delta \leq \frac{1}{2}$ we consider initial conditions $\left(\vec{p}^{-}, \vec{q}^{-}\right) \in \partial \mathcal{D}_{l} \cap \Sigma_{E}$ leading to a scattering angle $\Delta \psi:=\varangle\left(\vec{v}^{+}, \vec{v}^{-}\right)>c_{1} E^{-\delta}$. Then the linearized Kepler Transformation of Lemma 8.3 is estimated by

$$
\left(\begin{array}{c}
\delta \vec{v}^{+} \\
\delta \vec{w}^{+}
\end{array}\right)=\frac{4 c_{q} \sin ^{2}\left(\frac{1}{2} \Delta \psi\right)}{-Z_{l}} E \cdot\left(\begin{array}{ll}
R & R \\
R & R
\end{array}\right)\left(\begin{array}{c}
\delta \vec{v}^{-} \\
\delta \vec{w}^{-}
\end{array}\right)+\left|\left(\begin{array}{c}
\delta \vec{v}^{-} \\
\delta \vec{w}^{-}
\end{array}\right)\right| \cdot \mathcal{O}\left(E^{0}\right)
$$

where $R \equiv R_{\vec{v}^{+}-\vec{v}^{-}} \in O(3, \mathbb{R})$ is the reflection by the plane perpendicular to the vector $\vec{v}^{+}-\vec{v}^{-}$.

2) If instead $\Delta \psi<c_{2} E^{-\delta}$ with $\frac{1}{2} \leq \delta \leq 1$, then

$$
\begin{aligned}
\delta \vec{v}^{+} & =\delta \vec{v}^{-}+\quad \mathcal{O}\left(c_{2}^{2} E^{1-2 \delta}\right) \cdot\left(\left|\delta \vec{v}^{-}\right|+\left|\delta \vec{w}^{-}\right|\right), \\
\delta \vec{w}^{+} & =2 u \delta \vec{v}^{-}+\delta \vec{w}^{-}+2 \vec{v}^{-} d u+\mathcal{O}\left(c_{2}^{2} E^{1-2 \delta}\right) \cdot\left(\left|\delta \vec{v}^{-}\right|+\left|\delta \vec{w}^{-}\right|\right) .
\end{aligned}
$$


Remark 8.5. For $\delta \neq \frac{1}{2}$ the constants $c_{1}, c_{2}$ become irrelevant (for a threshold energy $E_{\text {th }} \geq 1$ ).

Observe that the leading term in (8.9) scales like $c_{1}^{2} E^{1-2 \delta} \gg 1$, whereas the error term of (8.10) scales like $c_{2}^{2} E^{1-2 \delta} \ll 1$.

Proof. 1) First we prove the identity

$$
1-u^{2}=\frac{e^{2}}{(2 / \beta+1)^{2}}
$$

with the eccentricity

$$
e=\sqrt{1+2 E\left|\vec{L}_{l}\right|^{2} / Z_{l}^{2}}=1 / \sin \left(\frac{1}{2} \Delta \psi\right)
$$

of the Kepler hyperbola. By reinserting the $(\vec{p}, \vec{q})$-coordinates with the help of (8.3), def. (8.5) of $u$ acquires the form $u=-2 \frac{\left\langle\vec{p}^{-}, \vec{q}^{-}\right\rangle}{\sqrt{2 E} c_{q}(2+\beta)}$ (assuming without loss of generality that $\vec{s}_{l}=\overrightarrow{0}$ ). We now insert the relationship

$$
\left\langle\vec{p}^{-}, \vec{q}^{-}\right\rangle^{2}=\left|\vec{p}^{-}\right|^{2}\left|\vec{q}^{-}\right|^{2}-\vec{L}_{l}^{2}=2\left(E+Z_{l} / c_{q}\right) c_{q}^{2}-\vec{L}_{l}^{2}
$$

into the expression for $1-u^{2}$ to deduce (8.11).

If $\Delta \psi>c E^{-\delta}$,

$$
1-u^{2}=\mathcal{O}\left(c^{2} E^{2(\delta-1)}\right)
$$

so that

$$
\delta \vec{v}^{+}=-\frac{2 \beta\left(\vec{v}^{-}+\vec{w}^{-}\right) d u}{\left(1-u^{2}\right)^{2}}-\frac{\beta\left(\delta \vec{v}^{-}+\delta \vec{w}^{-}\right)}{1-u^{2}}+\mathcal{O}\left(E^{0}\right) \cdot\left(\left|\delta \vec{v}^{-}\right|+\left|\delta \vec{w}^{-}\right|\right)
$$

and

$$
\delta \vec{w}^{+}=-\frac{2 \beta\left(\vec{v}^{-}+\vec{w}^{-}\right) d u}{\left(1-u^{2}\right)^{2}}-\frac{\beta\left(\delta \vec{v}^{-}+\delta \vec{w}^{-}\right)}{1-u^{2}}+\mathcal{O}\left(E^{0}\right) \cdot\left(\left|\delta \vec{v}^{-}\right|+\left|\delta \vec{w}^{-}\right|\right) .
$$

Using (8.8) we get

$$
d u=-\frac{\left\langle\vec{w}^{-}+\vec{v}^{-}, \delta \vec{v}^{-}+\delta \vec{w}^{-}\right\rangle}{1+\frac{1}{2} \beta} .
$$

The vectors $\vec{w}^{-}$and $\vec{v}^{-}$in the last formula are nearly anti-parallel. From the expression (8.4) for $\vec{v}^{+}$and (8.13) we see that $\vec{v}^{+}=\vec{v}^{-}-\frac{\beta\left(\vec{v}^{-}+\vec{w}^{-}\right)}{1-u^{2}}+\mathcal{O}(1 / E)$ or

$$
\vec{v}^{-}+\vec{w}^{-}=\left(1-u^{2}\right)\left(\frac{\vec{v}^{-}-\vec{v}^{+}}{\beta}+\mathcal{O}(1)\right)
$$


so that

$$
\begin{aligned}
\delta \vec{v}^{+}= & \frac{2\left(\vec{v}^{-}-\vec{v}^{+}+\mathcal{O}(1 / E)\right)\left\langle\vec{v}^{-}-\vec{v}^{+}+\mathcal{O}(1 / E), \delta \vec{v}^{-}+\delta \vec{w}^{-}\right\rangle}{\beta(1+\beta / 2)} \\
& -\frac{\beta\left(\delta \vec{v}^{-}+\delta \vec{w}^{-}\right)}{1-u^{2}}+\mathcal{O}\left(E^{0}\right) \cdot\left(\left|\delta \vec{v}^{-}\right|+\left|\delta \vec{w}^{-}\right|\right) \\
= & \frac{2\left(\vec{v}^{-}-\vec{v}^{+}\right)\left\langle\vec{v}^{-}-\vec{v}^{+}, \delta \vec{v}^{-}+\delta \vec{w}^{-}\right\rangle}{\beta}-\frac{\beta\left(\delta \vec{v}^{-}+\delta \vec{w}^{-}\right)}{1-u^{2}} \\
& +\mathcal{O}\left(E^{0}\right) \cdot\left(\left|\delta \vec{v}^{-}\right|+\left|\delta \vec{w}^{-}\right|\right)
\end{aligned}
$$

Extracting the square of the norm

$$
\begin{aligned}
\left|\vec{v}^{-}-\vec{v}^{+}\right| & =\left|\frac{\vec{v}^{-}}{\left|\vec{v}^{-}\right|}-\frac{\vec{v}^{+}}{\left|\vec{v}^{+}\right|}\right|+\mathcal{O}(1 / E) \\
& =\sqrt{2(1-\cos (\Delta \psi))}+\mathcal{O}(1 / E)=2 \sin \left(\frac{1}{2} \Delta \psi\right)+\mathcal{O}(1 / E),
\end{aligned}
$$

we obtain the first estimate in (8.9), since by (8.11) and (8.12)

$$
\frac{\beta}{1-u^{2}}=\frac{4}{\beta e^{2}}+\mathcal{O}\left(E^{0}\right)=\frac{4 \sin ^{2}\left(\frac{1}{2} \Delta \psi\right)}{\beta}+\mathcal{O}\left(E^{0}\right),
$$

and the reflection equals

$$
R_{\vec{v}^{+}-\vec{v}^{-}}=11-2 \frac{\left|\vec{v}^{-}-\vec{v}^{+}\right\rangle\left\langle\vec{v}^{-}-\vec{v}^{+}\right|}{\left|\vec{v}^{-}-\vec{v}^{+}\right|^{2}} .
$$

Estimate (8.9) for $\delta \vec{w}^{+}$follows since the r.h.s. of (8.15) and (8.14) have the same form.

2) We write

$$
\delta \vec{v}^{+}=\delta \vec{v}^{-}+R_{1}+R_{2} \quad, \quad \delta \vec{w}^{+}=2 u \delta \vec{v}^{-}+\delta \vec{w}^{-}+2 \vec{v}^{-} d u+R_{1}+R_{3}
$$

with rest terms

$$
\begin{gathered}
R_{1}:=-\frac{\beta}{1-u^{2}}\left[\delta \vec{v}^{-}+\delta \vec{w}^{-}+2 \frac{\left(\vec{v}^{-}+\vec{w}^{-}\right) d u}{1-u^{2}}\right], \\
R_{2}:=\beta\left[\delta \vec{v}^{-}+\frac{\delta \vec{w}^{-}}{1+u}+\frac{\vec{v}^{-} d u}{(1+u)^{2}}+\frac{\left(\vec{v}^{-}+\vec{w}^{-}\right) d u}{1-u^{2}}\right]
\end{gathered}
$$

and

$$
R_{3}:=\beta\left[\frac{1+u+u^{2}}{1+u} \delta \vec{v}^{-}-\delta \vec{w}^{-}+\vec{v}^{-} d u+\frac{\vec{w}^{-} d u}{(1+u)^{2}}-\frac{\left(\vec{v}^{-}+\vec{w}^{-}\right) d u}{1-u^{2}}\right]
$$

Now we assume that $\Delta \psi<c_{2} E^{-\delta}$ with $\frac{1}{2} \leq \delta \leq 1$.

So by the first part of the proof $R_{1}=\mathcal{O}\left(c_{2}^{2} E^{1-2 \delta}\right) \cdot\left(\left|\delta \vec{v}^{-}\right|+\left|\delta \vec{w}^{-}\right|\right)$. By the same reasoning the (identical, up to sign) last term in $R_{2}$ and $R_{3}$ is of order $\mathcal{O}(1 / E) \cdot\left(\left|\delta \vec{v}^{-}\right|+\left|\delta \vec{w}^{-}\right|\right)$. The other terms in $R_{2}$ and $R_{3}$ are of the same order, since they contain the multiplier $\beta$. 
We now use the results of Sect. 4 to compare the true motion with the Kepler motion of the last lemmata.

Proposition 8.6. For pericentric initial conditions $x_{0} \in \mathcal{H}_{l}$ with energy $E:=$ $H\left(x_{0}\right)>E_{\text {th }}$

$$
T_{l}^{ \pm}\left(x_{0}\right)=T_{L, l}^{ \pm}\left(x_{0}\right)+\mathcal{O}\left(E^{-3 / 2}\right)
$$

and for $\left(\vec{p}^{ \pm}, \vec{q}^{ \pm}\right):=\Psi_{l}^{ \pm}\left(x_{0}\right)$ and $\left(\vec{p}_{L}^{ \pm}, \vec{q}_{L}^{ \pm}\right):=\Psi_{L, l}^{ \pm}\left(x_{0}\right)$

$$
\left(\vec{p}^{ \pm} / \sqrt{2 E}, \vec{q}^{ \pm}\right)=\left(\vec{p}_{L}^{ \pm} / \sqrt{2 E}, \vec{q}_{L}^{ \pm}\right)+\mathcal{O}(1 / E)
$$

For $\left|\vec{q}_{0}-\vec{s}_{l}\right|=\mathcal{O}(1 / E)$

$$
T_{L, l}^{ \pm}\left(x_{0}\right)= \pm\left(\frac{c_{q}}{\sqrt{2 E}}-\frac{Z_{l}}{(2 E)^{3 / 2}} \ln \left(E c_{q} /\left|Z_{l}\right|\right)\right)+\mathcal{O}\left(E^{-3 / 2}\right) .
$$

For arbitrary $x_{0} \in \mathcal{H}_{l}$ the r.h.s. of (8.18) is an upper bound for $\left|T_{l}^{ \pm}\left(x_{0}\right)\right|$. The diffeomorphism

$$
\Xi_{l}: \partial \mathcal{D}_{l} \rightarrow \partial \mathcal{D}_{L, l}, \quad x \equiv(\vec{p}, \vec{q}) \mapsto\left\{\begin{array}{l}
\Psi_{L, l}^{-} \circ \Psi_{l}^{0}(x),\langle\vec{p}, \vec{q}\rangle \leq 0 \\
\Psi_{L, l}^{+} \circ \Psi_{l}^{0}(x),\langle\vec{p}, \vec{q}\rangle>0
\end{array}\right.
$$

onto its image which conjugates the maps $\left(\Psi_{l}=\left(\Xi_{l}\right)^{-1} \circ \Psi_{L, l} \circ \Xi_{l}\right)$ is $C^{0}$-near to the identity in the sense that in the Euclidean norm for the $(\vec{v}, \vec{w})$-coordinates $(8.3)$

$$
\left|\Xi_{l}(x)-x\right|=\mathcal{O}(1 / H(x)), \quad\left(x \in \partial \mathcal{D}_{l}\right),
$$

and the solution $\Psi_{L, l}$ of the linear problem is $C^{1}$-near to $\Psi_{l}$ in the sense

$$
\begin{aligned}
\left|\Psi_{l}(x)-\Psi_{L, l} \circ \Xi_{l}(x)\right| & =\mathcal{O}\left(H^{-1}(x)\right) \\
\left\|D \Psi_{l}(x)-D \Psi_{L, l} \circ \Xi_{l}(x)\right\| & =\mathcal{O}\left(H^{0}(x)\right) .
\end{aligned}
$$

Remark 8.7. In particular the exit times (8.18) are independent of $x_{0}$, up to $\mathcal{O}\left(E^{-3 / 2}\right)$, if $\left|\vec{q}_{0}-\vec{s}_{l}\right|=\mathcal{O}(1 / E)$.

Proof. Estimate (8.17) follows directly from (4.19).

Estimate (4.39) says that for KS initial conditions $X_{0}$ with $\pi\left(X_{0}\right)=x_{0}$ the relative error

$$
\frac{\left|\boldsymbol{\Phi}_{L, l}\left(s, X_{0}\right)-\boldsymbol{\Phi}_{l}\left(s, X_{0}\right)\right|}{\left|Q\left(s, X_{0}\right)\right|}=\mathcal{O}\left(E^{-1}\right) .
$$

This translates into the estimates

$$
\frac{\left|\vec{q}\left(t, x_{0}\right)-\vec{q}_{L}\left(t, x_{0}\right)\right|}{\left|\vec{q}\left(t, x_{0}\right)\right|}=\mathcal{O}\left(E^{-1}\right)
$$


and

$$
\frac{\left|\vec{p}\left(t, x_{0}\right)-\vec{p}_{L}\left(t, x_{0}\right)\right|}{\left|\vec{p}\left(t, x_{0}\right)\right|}=\mathcal{O}\left(E^{-1}\right) \quad(t \neq 0) .
$$

We know that inside $\mathcal{D}_{l}$ each trajectory $t \mapsto \Phi^{t}\left(x_{0}\right)$ passes the hypersurface $\mathcal{H}_{l}$ only at $x_{0}$, so that we can parametrize the curves $\left(0, T_{l}^{+}\left(x_{0}\right)\right] \ni t \mapsto \Phi^{t}\left(x_{0}\right) \equiv$ $(\vec{p}(t), \vec{q}(t))$ by their radius $r(t):=\left|\vec{q}(t)-\vec{s}_{l}\right|$ (similarly for negative times).

The explicit formula for the exit time

$$
T_{l}^{+}\left(x_{0}\right)=\frac{1}{\sqrt{2}} \int_{\left|\vec{q}_{0}\right|}^{c_{q}}\left(E+\frac{Z_{l}}{r}-\frac{\hat{L}_{l}^{2}(\vec{p}(r), \vec{q}(r))}{2 r^{2}}+W_{l}(\vec{q}(r))\right)^{-\frac{1}{2}} d r
$$

(with the smooth potential $W_{l}$ defined in (4.1), and relative angular momentum $\hat{L}_{l}$ in (5.6)) is obtained by integrating the inverse of the radial velocity $\langle\vec{p}, \vec{q}\rangle /|\vec{q}|$. Similarly

$$
T_{L, l}^{+}\left(x_{0}\right)=\frac{1}{\sqrt{2}} \int_{\left|\vec{q}_{0}\right|}^{c_{q}}\left(E+\frac{Z_{l}}{r}-\frac{L_{l}^{2}\left(x_{0}\right)}{2 r^{2}}\right)^{-\frac{1}{2}} d r .
$$

inserting (8.22) and (8.23) then shows the assertion (8.16).

(8.18) follows by evaluating the explicit formula (5.9) for the exit time of the Kepler flow: As $E r_{\min }^{2}+Z_{l} r_{\min }-\frac{1}{2} L_{l}^{2}=0$, the square root terms in (5.9) vanish for the minimal radius. Further, by our assumption on $\vec{q}_{0}$ we have $r_{\min } \leq C\left|Z_{l}\right| / E$ for some $C>0$, so that

$$
L_{l}^{2} \leq\left|\vec{q}-\vec{s}_{l}\right|^{2} \vec{p}^{2}=2\left|\vec{q}-\vec{s}_{l}\right|\left(E\left|\vec{q}-\vec{s}_{l}\right|+Z_{l}\right) \leq \frac{2 Z_{l}^{2} C(1+C)}{E}=\mathcal{O}(1 / E) .
$$

Est. (8.19) follows from (4.19) and similarly (8.20), (8.21) follow from (4.20) and (4.21), since on $\partial \mathcal{D}_{L, l}$ the KS transformation from coordinates $(\tilde{P}, Q)$ to the $(\vec{v}, \vec{w})$-coordinates $(8.3)$ takes the simple form

$$
\left(\begin{array}{c}
\vec{v} \\
\vec{w}
\end{array}\right)=\left(\begin{array}{l}
Q^{*} I_{3} \tilde{P} \\
Q^{*} I_{3} Q
\end{array}\right) / c_{q}
$$

\section{Long paths within the interaction zone}

Strong changes of directions only occur if the pericentric distance of the orbit from a nucleus is of order $\mathcal{O}(1 / E)$.

To quantify this, we set for $0<\theta \leq \pi$

$$
\mathcal{H}_{l}(\theta):=\left\{x \in \mathcal{H}_{l}|| \vec{q}(x)-\vec{s}_{l} \mid<\frac{\left|Z_{l}\right|}{H(x) \cdot \sin (\theta / 4)}\right\}
$$

(with the pericentric hypersurface $\mathcal{H}_{l}$ defined in (8.1)). 
Lemma 9.1. For $C_{5}>0$ large the following statements are true. Consider pericentric initial conditions $x_{0} \in \mathcal{H}_{l}$ of energy $E:=H\left(x_{0}\right)>E_{\mathrm{th}}$ and $\theta \in\left[C_{5} / E, \pi\right]$.

- If $x_{0} \notin \mathcal{H}_{l}(\theta)$, then the directions $\hat{p}^{ \pm}:=\hat{p}\left(\Psi_{l}^{ \pm}\left(x_{0}\right)\right)$ of the orbit through $x_{0}$ at the moment of entering, resp. exit from the ball $B_{l}\left(c_{q}\right)$ differ at most by the angle

$$
\Delta \psi=\varangle\left(\hat{p}^{-}, \hat{p}^{+}\right)<\theta .
$$

- Conversely, if $x_{0} \in \mathcal{H}_{l}(\theta)$, then

$$
\Delta \psi \geq \theta / 4
$$

and positions and momenta are nearly anti-parallel before scattering and parallel after scattering:

$$
\varangle\left(\vec{q}\left(\Psi_{l}^{ \pm}\left(x_{0}\right)\right)-\vec{s}_{l}, \pm \hat{p}^{ \pm}\right)=\mathcal{O}\left((\theta E)^{-1}\right) .
$$

Proof. Because of estimate (8.20) of Proposition 8.6 we need only choose $C_{5}$ large and then show the following statements for the Kepler flow (which correspond to $(9.2),(9.3)$ resp. $(9.4))$ :

$$
\begin{gathered}
\Delta \psi_{L}:=\varangle\left(\hat{p}_{L}^{-}, \hat{p}_{L}^{+}\right) \leq \frac{1}{2} \theta, \\
\sin \left(\frac{1}{2} \Delta \psi_{L}\right) \geq 1.05 \cdot \sin (\theta / 8)
\end{gathered}
$$

and

$$
\varangle\left(\vec{q}_{L}^{ \pm}-\vec{s}_{l}, \pm \hat{p}_{L}^{ \pm}\right)=\mathcal{O}\left((\theta E)^{-1}\right)
$$

with $\hat{p}_{L}^{ \pm}:=\hat{p}\left(\Psi_{L, l}^{ \pm}\left(x_{0}\right)\right)$ and $\vec{q}_{L}^{ \pm}:=\vec{q}\left(\Psi_{L, l}^{ \pm}\left(x_{0}\right)\right)$.

- We use the explicit formulae of Lemma 8.1 to compute

$$
\begin{aligned}
\cos \left(\Delta \psi_{L}\right) & =\frac{\left\langle\vec{v}^{-}, \vec{v}^{+}\right\rangle}{\left|\vec{v}^{-}\right|\left|\vec{v}^{+}\right|}=\frac{\left\langle\vec{v}^{-}, \vec{v}^{+}\right\rangle}{1+\beta}=1-\frac{1}{2} \frac{u^{2} \beta^{2}}{\left(1-u^{2}\right)(1+\beta)} \\
& =1-\frac{1}{2} \frac{u^{2}(2+\beta)^{2}}{e^{2}(1+\beta)}
\end{aligned}
$$

using the substitution (8.11). Now the eccentricity $e$ of the Kepler hyperbola equals

$$
e=\left|1+2 E r_{\min } / Z_{l}\right|,
$$

as one sees by comparing the definition (5.8) of the pericentric distance $r_{\text {min }}$ with (8.12) (formula (5.8) is also valid if $Z_{l}<0$; then the energy is always positive). Thus

$$
\begin{aligned}
\sin \left(\frac{1}{2} \Delta \psi_{L}\right) & =\sqrt{\frac{1}{2}\left(1-\cos \left(\Delta \psi_{L}\right)\right)}=\frac{u\left(1+\frac{1}{2} \beta\right)}{\sqrt{1+\beta}} \frac{1}{\left|2 E r_{\min } / Z_{l}+1\right|} \\
& \leq\left|2 E r_{\min } / Z_{l}+1\right|^{-1} \leq \frac{\sin \left(\frac{1}{4} \theta\right)}{2+\sin \left(\frac{1}{4} \theta\right) \operatorname{sign}\left(Z_{l}\right)} \leq \sin \left(\frac{1}{4} \theta\right)
\end{aligned}
$$


since $u\left(1+\frac{1}{2} \beta\right) / \sqrt{1+\beta}$ equals the cosine of the angle between $\vec{v}^{+}$and $\vec{w}^{+}$and is thus smaller than one, see (8.5). Then (9.5) follows, since the sine function is monotone increasing on $[0, \pi / 2]$.

- By (8.12) and (9.8)

$$
\begin{aligned}
\sin \left(\frac{1}{2} \Delta \psi_{L}\right) & =1 / e=\left|1+2 E r_{\min } / Z_{l}\right|^{-1} \geq \frac{\sin (\theta / 4)}{\left|1+\operatorname{sign}\left(Z_{l}\right) \sin (\theta / 4)\right|} \\
& \geq \frac{\sin (\theta / 4)}{1+1 / \sqrt{2}} \geq \frac{\sin (\pi / 4)}{2 \sin (\pi / 8)} \frac{2 \sin (\theta / 8)}{1+1 / \sqrt{2}}
\end{aligned}
$$

showing (9.6).

- With (8.5), (8.11) and (9.8)

$$
\begin{aligned}
\sin & \left(\varangle\left(\vec{q}\left(\Psi_{l}^{ \pm}\left(x_{0}\right)\right)-\vec{s}_{l}, \pm \hat{p}^{ \pm}\right)\right)=\sin \left(\varangle\left(\vec{w}^{ \pm}, \pm \vec{v}^{ \pm}\right)\right)=\sqrt{1-\frac{\left\langle\vec{w}^{ \pm}, \pm\left.\vec{v}^{ \pm}\right|^{2}\right.}{\left\langle\vec{w}^{ \pm}, \vec{w}^{ \pm}\right\rangle}} \\
& =\sqrt{1-u^{2} \frac{\left(1+\frac{1}{2} \beta\right)^{2}}{1+\beta}}=\sqrt{\left|1-u^{2}\right|}+\mathcal{O}(1 / E) \\
& =\frac{e}{|2 / \beta+1|}+\mathcal{O}(1 / E)=\left|\frac{1+2 E r_{\min } / Z_{l}}{2 / \beta+1}\right|+\mathcal{O}(1 / E) \\
& =\left|\frac{2 r_{\min } / Z_{l}+1 / E}{2 c_{q} / Z_{l}+1 / E}\right|+\mathcal{O}(1 / E)=\frac{r_{\min }}{c_{q}}+\mathcal{O}(1 / E)=\mathcal{O}(1 /(\theta E)),
\end{aligned}
$$

showing estimate (9.7).

Long trajectories in the interaction zone $\mathcal{I Z}$ must have close encounters with singularities of distance $\mathcal{O}(1 / E)$. To show this, we now assume that the radius $c_{q}$ of the balls $B_{k}\left(c_{q}\right)$ is so small that there is no straight line meeting more than two balls. To be concrete, we assume

$$
c_{q} \leq \frac{1}{4} \sin \left(\alpha_{\min }\right) \cdot d_{\min } .
$$

Proposition 9.2. For $E_{\mathrm{th}}$ large we consider a trajectory segment

$$
[0, T] \ni t \mapsto \Phi^{t}\left(x_{0}\right) \equiv(\vec{p}(t), \vec{q}(t)) \in \mathcal{D}
$$

with

$$
\mathcal{D}:=\left\{x \in P \mid H(x)>E_{\text {th }}, \vec{q}(x) \in \mathcal{I Z}\right\},
$$

which does not intersect any pericentric hypersurface $\mathcal{H}_{l}\left(\alpha_{\min } / 2\right),(l=1, \ldots, n)$. Then, in configuration space, it does not reenter a ball $B_{k}\left(c_{q}\right)$ after leaving it, and it does not intersect three or more such balls.

Furthermore, the length of the time interval is bounded by $T<13 R_{\mathrm{vir}} / \sqrt{2 E}$. 
Proof. - There is no subsegment $\left[t_{1}, t_{2}\right] \ni t \mapsto \vec{q}(t)$ of the trajectory, lying in $\mathcal{I Z}\left(c_{q}\right)$, which leaves and then reenters a given ball $B_{k}\left(c_{q}\right)$. Namely we may otherwise assume

$$
\vec{q}\left(\left[t_{1}, t_{2}\right]\right) \subset \mathcal{I Z}\left(c_{q}\right) \quad, \quad\left|\vec{q}\left(t_{1}\right)-\vec{s}_{k}\right|=\left|\vec{q}\left(t_{2}\right)-\vec{s}_{k}\right|=c_{q} \quad \text { and } \quad t_{2}>t_{1} .
$$

Then there would be a time $t_{0} \in\left(t_{1}, t_{2}\right)$ with maximal distance $\left|\vec{q}\left(t_{0}\right)-\vec{s}_{k}\right| \geq c_{q}$ from $\vec{s}_{k}$ :

$$
\frac{d}{d t}\left|\vec{q}\left(t_{0}\right)-\vec{s}_{k}\right|^{2}=0 \quad \text { and } \quad \frac{d^{2}}{d t^{2}}\left|\vec{q}\left(t_{0}\right)-\vec{s}_{k}\right|^{2} \leq 0
$$

But

$$
\begin{aligned}
\frac{1}{2} \frac{d^{2}}{d t^{2}} \mid \vec{q}\left(t_{0}\right) & -\left.\vec{s}_{k}\right|^{2}=\left|\vec{p}\left(t_{0}\right)\right|^{2}-\left\langle\nabla V\left(\vec{q}\left(t_{0}\right)\right), \vec{q}\left(t_{0}\right)-\vec{s}_{k}\right\rangle \\
& \geq 2\left(E-L_{0}\right)-\sup _{\vec{q} \in \mathcal{I} \mathcal{Z}\left(c_{q}\right)}\left\langle\nabla V(\vec{q}), \vec{q}-\vec{s}_{k}\right\rangle \geq 2\left(E-L_{0}-L_{1} R_{\mathrm{vir}}\right)
\end{aligned}
$$

with $L_{0}=\sup _{\vec{q} \in \mathcal{I Z}\left(c_{q}\right)}|V(\vec{q})|$ and $L_{1}=\sup _{\vec{q} \in \mathcal{I Z}\left(c_{q}\right)}|\nabla V(\vec{q})|$, so that (9.11) does not hold for $E$ large.

- We now prove that there is no orbit segment (9.10) whose configuration space projection intersects three balls $B_{k_{0}}\left(c_{q}\right), B_{k_{1}}\left(c_{q}\right)$ and $B_{k_{2}}\left(c_{q}\right)$ in succession. By going to a subsegment, we otherwise assume outgoing initial data

$$
\vec{q}(0) \in \partial B_{k_{0}}\left(c_{q}\right) \quad, \quad\left\langle\vec{p}(0), \vec{q}(0)-\vec{s}_{k_{0}}\right\rangle \geq 0,
$$

ingoing final data

$$
\vec{q}(T) \in \partial B_{k_{2}}\left(c_{q}\right) \quad, \quad\left\langle\vec{p}(T), \vec{q}(T)-\vec{s}_{k_{2}}\right\rangle \leq 0,
$$

and intermediate times

$0<t^{-} \leq t^{+}<T \quad$ with $\quad \vec{q}\left(t^{ \pm}\right) \in \partial B_{k_{1}}\left(c_{q}\right) \quad$ and $\quad \pm\left\langle\vec{p}\left(t^{ \pm}\right), \vec{q}\left(t^{ \pm}\right)-\vec{s}_{k_{1}}\right\rangle \geq 0$

By the first part of the proof we know that $k_{0} \neq k_{1} \neq k_{2}$. Then the angle $\gamma$ between the direction $\hat{s}^{k_{0}, k_{1}}$ of the axis through the centres and $\hat{p}\left(t^{-}\right)$is bounded by

$$
\gamma<\frac{1}{2} \alpha_{\min }
$$

Namely all straight lines intersecting $B_{k_{0}}\left(c_{q}\right)$ and $B_{k_{1}}\left(c_{q}\right)$ have a direction whose angle $\gamma^{\prime}$ with $\hat{s}^{k_{0}, k_{1}}$ is bounded by

$$
\sin \left(\gamma^{\prime}\right) \leq \frac{c_{q}}{\frac{1}{2} d_{\min }} \leq \frac{1}{2} \sin \left(\alpha_{\min }\right)<\sin \left(\frac{1}{2} \alpha_{\min }\right)
$$

using (9.9), so that (9.12) follows for $E_{\text {th }}$ large from Lemma 7.1.

We now consider the unit vector $\hat{u}=\lambda_{0} \hat{s}^{k_{0}, k_{1}}+\lambda_{2} \hat{s}^{k_{2}, k_{1}}, \lambda_{i} \geq 0$, which is perpendicular to $\vec{s}^{k_{0}, k_{2}}$. 
Then by (9.12) the angle between $\hat{p}\left(t^{-}\right)$and $\hat{u}$ is bounded above by

$$
\begin{aligned}
\varangle\left(\hat{p}\left(t^{-}\right), \hat{u}\right) & \leq \varangle\left(\hat{s}^{k_{0}, k_{1}}, \hat{u}\right)+\gamma=\pi-\frac{1}{2} \pi-\alpha\left(k_{1}, k_{0}, k_{2}\right)+\gamma \\
& \leq \frac{1}{2} \pi-\alpha_{\min }+\gamma<\frac{1}{2}\left(\pi-\alpha_{\min }\right)
\end{aligned}
$$

(with $\cos (\alpha(i, j, k))=\left\langle\hat{s}^{j, i}, \hat{s}^{j, k}\right\rangle$ ).

On the other hand for some time $t \in\left[t^{+}, T\right]$ we have

$$
\langle\hat{p}(t), \hat{u}\rangle \leq\left\langle\frac{\vec{q}(T)-\vec{q}\left(t^{+}\right)}{\left|\vec{q}(T)-\vec{q}\left(t^{+}\right)\right|}, \hat{u}\right\rangle<0,
$$

because otherwise there is no trajectory between $\vec{q}\left(t^{+}\right)$and $\vec{q}(T)$.

But by Lemma 7.1 in the interval $t \in\left[t^{+}, T\right]$ the change of direction

$$
\varangle\left(\hat{p}\left(t^{+}\right), \hat{p}(t)\right)=\mathcal{O}(1 / E),
$$

so that with (9.13) and (9.14) we must have a large scattering angle

$$
\varangle\left(\hat{p}\left(t^{-}\right), \hat{p}\left(t^{+}\right)\right) \geq \frac{1}{2} \alpha_{\min } .
$$

within $B_{k_{1}}\left(c_{q}\right)$.

Our assumption that the orbit segment (9.10) does not intersect the pericentric hypersurface $\mathcal{H}_{k_{1}}\left(\alpha_{\min } / 2\right)$ (see (9.1) implies by Lemma 9.1 that the deflection angle is bounded above by

$$
\varangle\left(\hat{p}\left(t^{-}\right), \hat{p}\left(t^{+}\right)\right)<\frac{1}{2} \alpha_{\min }
$$

contradicting (9.15)

Thus a trajectory segment can be represented as the union of at most two segments inside balls $B_{k_{i}}\left(c_{q}\right)$ and three segments in $\mathcal{I Z}\left(c_{q}\right)$.

- By (8.16) and (8.18) for large $E_{\text {th }}$ the time interval spent by the trajectory inside the ball $B_{k_{i}}\left(c_{q}\right)$ has length $T_{k_{i}}^{+}-T_{k_{i}}^{-} \leq \frac{4 c_{q}}{\sqrt{2 E}}<\frac{2 R_{\mathrm{vir}}}{\sqrt{2 E}}$. By Lemma 7.1 with (7.1) the time interval of the segments in $\mathcal{I Z}\left(c_{q}\right)$ are of lengths $\leq 3 R_{\mathrm{vir}} / \sqrt{2 E}$. So the total time $T$ is bounded above by

$$
T<2 \frac{2 R_{\mathrm{vir}}}{\sqrt{2 E}}+3 \frac{3 R_{\mathrm{vir}}}{\sqrt{2 E}}=13 \frac{R_{\mathrm{vir}}}{\sqrt{2 E}} .
$$

We now control the paths between successive close encounters with the centres $\vec{s}_{k}$. For radii $c_{q}>0$ meeting (9.9) the balls $B_{k}\left(c_{q}\right), k \in\{1, \ldots, n\}$ around the centres $\vec{s}_{k}$ do not intersect.

By the NC condition 2.4 the distance $\operatorname{dist}\left(\vec{s}_{i}, A^{k, l}\right), i \neq k, l$ between the centre and the axis

$$
A^{k, l}:=\left\{t \vec{s}_{l}+(1-t) \vec{s}_{k} \mid t \in[0,1]\right\}, \quad(k \neq l)
$$

connecting $\vec{s}_{k}$ and $\vec{s}_{l}$ is bounded below by $\sin \left(\alpha_{\min }\right) \cdot d_{\min }$. 
Thus the configuration space cylinders (see Fig. 1)

$$
\mathcal{Z}_{M}^{k, l}:=\left\{\vec{q} \in \mathbb{R}_{\vec{q}}^{3}\left|\operatorname{dist}\left(\vec{q}, A^{k, l}\right) \leq c_{y},\right| \vec{q}-\vec{s}_{k}\left|\geq c_{q} \leq\right| \vec{q}-\vec{s}_{l} \mid\right\}
$$

have empty intersection with non-adjacent balls $\left(\mathcal{Z}_{M}^{k, l} \cap B_{i}\left(c_{q}\right)=\emptyset\right.$ for $\left.i \neq k, l\right)$ if $c_{y} \leq c_{q}$.

- The cylinders themselves are not mutually disjoint; in particular $\mathcal{Z}_{M}^{l, k}=\mathcal{Z}_{M}^{k, l}$.

- However, under the NC condition

$$
\mathcal{Z}_{M}^{k_{1}, l_{1}} \cap \mathcal{Z}_{M}^{k_{2}, l_{2}}=\varnothing
$$

if the axes $A^{k_{1}, l_{1}} \neq A^{k_{2}, l_{2}}$ are parallel.

- For the choice

$$
c_{y}:=\frac{1}{2} \sin \left(\alpha_{\min } / 2\right) c_{q}
$$

of the radius we have in addition

$$
\mathcal{Z}_{M}^{k, l_{1}} \cap \mathcal{Z}_{M}^{k, l_{2}}=\emptyset \quad\left(l_{1} \neq l_{2}\right) .
$$

- Finally there is a minimal nonzero angle between nonparallel axes. More precisely, for this choice of $c_{y}$ a cylinder $\mathcal{Z}_{M}^{k_{1}, l_{1}}$ can only intersect a different $\left(\left\{k_{1}, l_{1}\right\} \neq\left\{k_{2}, l_{2}\right\}\right)$ cylinder $\mathcal{Z}_{M}^{k_{2}, l_{2}}$ if the angle $\arccos \left(\left|\left\langle\hat{s}^{k_{1}, l_{1}}, \hat{s}^{k_{2}, l_{2}}\right\rangle\right|\right)$ between their axes is larger than $\alpha_{\min }$. The proof of this fact is a nice exercise in conic sections.

Thus for

$$
c_{p}:=\frac{1}{2} \min \left(\frac{1}{2} \alpha_{\min }, c_{y} / d_{\max }\right)
$$

and $E>E_{\mathrm{th}}$ the phase space regions

$$
\mathcal{Z}^{k, l}:=\left\{x \equiv(\vec{p}, \vec{q}) \in \mathcal{D}\left|\vec{q} \in \mathcal{Z}_{M}^{k, l},\right| \vec{p} / \sqrt{2 H(x)}-\hat{s}^{k, l} \mid \leq c_{p}\right\}
$$

(with $\left.\mathcal{D}=\left\{x \in P \mid H(x)>E_{\text {th }}, \vec{q}(x) \in \mathcal{I Z}\right\}\right)$ do not intersect $\left(\mathcal{Z}^{k_{1}, l_{1}} \cap \mathcal{Z}^{k_{2}, l_{2}}=\emptyset\right.$ for $\left.\left(k_{1}, l_{1}\right) \neq\left(k_{2}, l_{2}\right)\right)$.

For $k \neq l$ we erect Poincaré hypersurfaces $\mathcal{H}^{k, l}$ near the midpoint

$$
\vec{m}^{k, l}:=\frac{1}{2}\left(\vec{s}_{k}+\vec{s}_{l}\right) \in A^{k, l}
$$

by setting for $e_{0}>0$

$$
\mathcal{H}_{M}^{k, l}(E):=\left\{\vec{q} \in \mathbb{R}_{\vec{q}}^{3}\left|\left\langle\vec{q}-\vec{m}^{k, l}, \hat{s}^{k, l}\right\rangle=0,\right| \vec{q}-\vec{m}^{k, l} \mid<\frac{1}{8} c_{p} \cdot d^{k, l} e_{0} / E\right\},
$$

so that $\mathcal{H}_{M}^{k, l}(E) \subset \mathcal{Z}_{M}^{k, l}$ for $E_{\text {th }}$ large and $E>E_{\text {th }}$, and

$$
\begin{aligned}
\mathcal{H}_{E}^{k, l}:=\left\{(\vec{p}, \vec{q}) \in \Sigma_{E} \mid \vec{q} \in \mathcal{H}_{M}^{k, l}(E), .\right. \\
\left.\left.\qquad \vec{p}, \hat{s}^{k, l}\right\rangle>0,\left|\frac{\vec{p}}{\sqrt{2 E}} \times \hat{s}^{k, l}\right|<\frac{1}{2} c_{p} e_{0} / E\right\},
\end{aligned}
$$




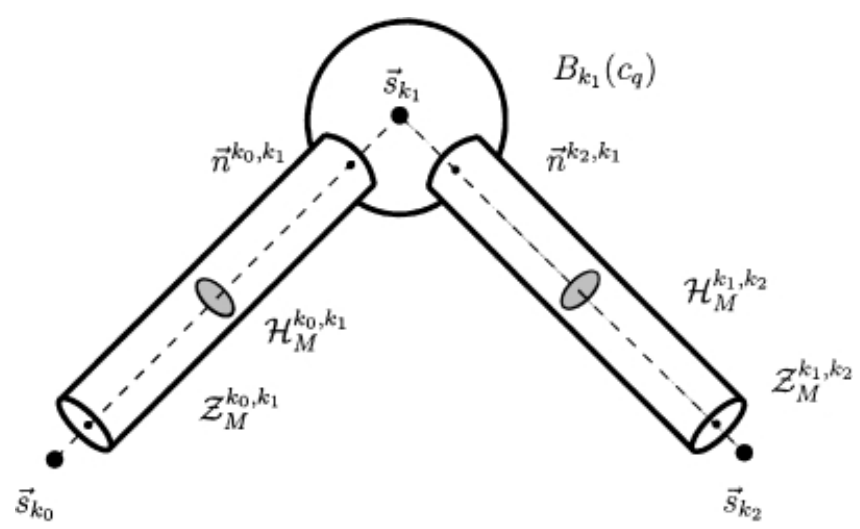

Fig. 1. Configuration space projections $\mathcal{H}_{M}^{k, l}$ of Poincaré sections

so that $\mathcal{H}_{E}^{k, l} \subset \mathcal{Z}^{k, l}$, too; see Fig. 1. The $\mathcal{H}_{E}^{k, l}$ are four-dimensional submanifolds without boundary of $\Sigma_{E}$. Sometimes we work with their closures

$$
\overline{\mathcal{H}}_{E}^{k, l}
$$

which are diffeomorphic to products of two closed disks (and as such formally speaking no manifolds).

High energy orbits between near-collisions are nearly straight and thus move near some axis:

Lemma 9.3. Let $E_{\mathrm{th}}$ and the constant $e_{0}>0$ in (9.16) be large and consider for $E>E_{\text {th }}$ trajectory segments of the form

$$
[-T, T] \ni t \mapsto x(t) \equiv(\vec{p}(t), \vec{q}(t)):=\Phi^{t}\left(x_{0}\right)
$$

with initial values $x_{0} \in \Sigma_{E}$, starting and ending in the pericentric hypersurfaces (9.1):

$$
x( \pm T) \in \mathcal{H}_{k_{ \pm}}\left(\alpha_{\min } / 2\right)
$$

but not intersecting a pericentric hypersurfaces in between:

$$
x((-T, T)) \cap \mathcal{H}_{l}\left(\alpha_{\min } / 2\right)=\emptyset \quad(l=1, \ldots, n) .
$$

Then the trajectory segment is contained in the phase space region

$$
x([-T, T]) \subset \mathcal{Z}^{k_{-}, k_{+}} \cup \mathcal{D}_{k_{-}} \cup \mathcal{D}_{k_{+}},
$$

and there is a unique $t_{0} \in(-T, T)$ with

$$
\Phi^{t_{0}}\left(x_{0}\right) \in \mathcal{H}_{E}^{k_{-}, k_{+}} .
$$

Conversely for $k_{+} \neq k_{-}$the exit time

$$
T_{E}^{k_{-}, k_{+}, \pm}: \mathcal{H}_{E}^{k_{-}, k_{+}} \rightarrow \mathbb{R}^{ \pm}, T_{E}^{k_{-}, k_{+}, \pm}\left(x_{0}\right):= \pm \inf \left\{t \geq 0 \mid \Phi^{ \pm t}\left(x_{0}\right) \in \partial \mathcal{Z}^{k_{-}, k_{+}}\right\}
$$


from $\mathcal{Z}^{k_{-}, k_{+}}$is of order

$$
T_{E}^{k_{-}, k_{+}, \pm}= \pm \frac{\frac{1}{2} d^{k_{-}, k_{+}}-c_{q}}{\sqrt{2 E}}+\mathcal{O}\left(E^{-3 / 2}\right)
$$

and the exit points $\left(\vec{p}^{ \pm}, \vec{q}^{ \pm}\right):=\Phi\left(T_{E}^{k_{-}, k_{+}, \pm}\left(x_{0}\right), x_{0}\right)$ are estimated by

$$
\left(\vec{p}^{ \pm} / \sqrt{2 E}, \vec{q}^{ \pm}\right)=\left(\hat{s}^{k_{-}, k_{+}}, \vec{n}^{k_{\mp}, k_{ \pm}}\right)+\mathcal{O}(1 / E),
$$

with the intersection point $\vec{n}^{k, l}:=\vec{s}_{l}+c_{q} \hat{s}^{l, k}$ between the axis $A^{k, l}$ and the sphere $\partial B_{l}\left(c_{q}\right)$, see Fig. 1 .

Proof. By straight line geometry and Lemma 7.1 trajectories $t \mapsto \vec{q}\left(t, x_{0}\right)$ with $x_{0} \in \mathcal{H}_{E}^{k_{-}, k_{+}}$intersect $\partial \mathcal{Z}_{M}^{k_{-}, k_{+}}$near $\vec{n}^{k_{\mp}, k_{ \pm}}$, and

$$
\vec{p}^{ \pm} / \sqrt{2 E}-\hat{s}^{k_{-}, k_{+}}=\left(\vec{p}^{ \pm} / \sqrt{2 E}-\vec{p}_{0}\right)+\left(\vec{p}_{0}-\hat{s}^{k_{-}, k_{+}}\right)=\mathcal{O}(1 / E),
$$

again using Lemma 7.1 and the definition (9.16) of $\mathcal{H}_{E}^{k_{-}, k_{+}}$. This shows (9.22). Since $\left|\vec{m}^{k_{-}, k_{+}}-\vec{n}^{k_{\mp}, k_{ \pm}}\right|=\frac{1}{2} d^{k_{-}, k_{+}}-c_{q}$, (9.21) follows from Lemma 7.1.

Concerning the first statement of the lemma, we know from (9.4) of Lemma 9.1 that for $t^{ \pm}:=T_{k_{\mp}}^{ \pm}(x(\mp T))$

$$
\left(\vec{p}^{ \pm}, \vec{q}^{ \pm}\right):=\Phi\left(t^{\mp}, x( \pm T)\right) \in\left(\left.\vec{q}\right|_{\Sigma_{E}}\right)^{-1}\left(\partial B_{k_{ \pm}}\left(c_{q}\right)\right)
$$

have the property

$$
\vec{q}^{ \pm}-\vec{s}_{k_{ \pm}}= \pm \frac{c_{q}}{\sqrt{2 E}} \vec{p}^{ \pm}+\mathcal{O}(1 / E) .
$$

Furthermore, by the assumption (9.18) and the first statement of Proposition 9.2 the trajectory lies in $\mathcal{I Z}\left(c_{q}\right)$ during the time interval $\left[-T+t^{+}, T-t^{-}\right]$. Thus (7.2) and (7.1) imply that

$$
\frac{\vec{p}^{+}}{\sqrt{2 E}}=\frac{\vec{p}^{-}}{\sqrt{2 E}}+\mathcal{O}(1 / E)
$$

and

$$
\frac{\vec{q}^{+}-\vec{q}^{-}}{\left|\vec{q}^{+}-\vec{q}^{-}\right|}=\frac{\vec{p}^{-}}{\sqrt{2 E}}+\mathcal{O}(1 / E) .
$$

Using (9.23), (9.25) and then (9.24),

$$
\begin{aligned}
\vec{s}_{k_{+}-\vec{s}_{k_{-}}} \equiv\left(\vec{s}_{k_{+}}-\vec{q}^{+}\right)+\left(q^{-}-\vec{s}_{k_{-}}\right)+\left(\vec{q}^{+}-\vec{q}^{-}\right) \\
=\frac{c_{q}}{\sqrt{2 E}}\left(\vec{p}^{+}+\vec{p}^{-}\right)+\frac{\left|\vec{q}^{+}-\vec{q}^{-}\right|}{\sqrt{2 E}} \vec{p}^{-}+\mathcal{O}(1 / E) \\
=\frac{2 c_{q}+\left|\vec{q}^{+}-\vec{q}^{-}\right|}{\sqrt{2 E}} \vec{p}^{ \pm}+\mathcal{O}(1 / E)
\end{aligned}
$$

so that

$$
\left|\vec{p}^{ \pm} / \sqrt{2 E}-\hat{s}^{k_{-}, k_{+}}\right|=\mathcal{O}(1 / E) .
$$

A second application of (9.25) shows that the second term in (9.22), too is $\mathcal{O}(1 / E)$.

By Lemma 7.1 we obtain (9.19) and (9.20). 


\section{The Poincaré map}

For $E>E_{\text {th }}$ consider the Poincaré surfaces

$$
\mathcal{H}_{E}:=\bigcup_{\substack{k, l=1 \\ k \neq l}}^{n} \mathcal{H}_{E}^{k, l} \quad, \quad \mathcal{H}_{E}^{ \pm}:=\mathcal{H}_{E} \cup \partial \mathcal{D}_{E}^{ \pm}
$$

with $\mathcal{H}_{E}^{k, l}$ defined in (9.16) and

$$
\mathcal{D}_{E}:=\mathcal{D} \cap \Sigma_{E}, \quad \partial \mathcal{D}_{E}^{ \pm}:=\left\{(\vec{p}, \vec{q}) \in \partial \mathcal{D}_{E} \mid \pm\langle\vec{p}, \vec{q}\rangle \geq 0\right\} .
$$

Then the return time to the Poincare surface $\mathcal{H}_{E}^{+}$

$$
T_{E}: \mathcal{D}_{E} \rightarrow[0, \infty) \cup\{\infty\},
$$

is defined by $T_{E}(x):=0$ for $x \in \partial \mathcal{D}_{E}^{+}$and

$$
T_{E}(x):=\inf \left\{t>0 \mid \Phi_{E}^{t}(x) \in \mathcal{H}_{E}^{+}\right\}, \quad\left(x \in \mathcal{D}_{E} \backslash \partial \mathcal{D}_{E}^{+}\right) .
$$

Lemma 10.1. For $E>E_{\mathrm{th}}$ the Poincaré return time $T_{E}$ is finite and

$$
T_{E}=\mathcal{O}(1 / \sqrt{E}) .
$$

Proof. Let $x_{0} \in \mathcal{D}_{E} \backslash \partial \mathcal{D}_{E}^{+}$. By Proposition 9.2 for a time $t_{1} \in\left[0,13 R_{\mathrm{vir}} / \sqrt{2 E}\right]$ the point $x_{1}:=\Phi^{t_{1}}\left(x_{0}\right)$

- either exits $\mathcal{D}_{E}$, that is $x_{1} \in \partial \mathcal{D}_{E}^{+}$

- or meets a pericentric surface near the $k$ th nucleus: $x_{1} \in \mathcal{H}_{k}\left(\alpha_{\min } / 2\right)$.

In the first case we are done. In the second case we iterate the above argument and find $t_{2} \in\left[t_{1}, t_{1}+13 R_{\text {vir }} / \sqrt{2 E}\right]$ for which the point $x_{2}:=\Phi^{t_{2}}\left(x_{0}\right)$ either exits $\mathcal{D}_{E}$ $\left(x_{2} \in \partial \mathcal{D}_{E}^{+}\right)$or meets a pericentric surface near the $l \neq k$ th nucleus:

$$
x_{2} \in \mathcal{H}_{l}\left(\alpha_{\min } / 2\right) .
$$

In the relevant second case we know from (9.20) of Lemma 9.3 that

$$
x_{3}:=\Phi^{t_{3}}\left(x_{0}\right) \in \mathcal{H}_{E}^{k, l}
$$

for some $t_{3} \in\left(t_{1}, t_{2}\right)$.

We shall analyze the Poincaré map

$$
\mathcal{P}_{E}: \mathcal{H}_{E}^{-} \rightarrow \mathcal{H}_{E}^{+}, \quad \mathcal{P}_{E}(x):=\Phi\left(T_{E}(x), x\right) .
$$

When we work with the closures

$$
\overline{\mathcal{H}}_{E}:=\bigcup_{\substack{k, l=1 \\ k \neq l}}^{n} \overline{\mathcal{H}}_{E}^{k, l} \quad, \quad \overline{\mathcal{H}}_{E}^{ \pm}:=\overline{\mathcal{H}}_{E} \cup \partial \mathcal{D}_{E}^{ \pm}
$$

(see (9.17)), we write $\bar{T}_{E}, \overline{\mathcal{P}}_{E}$ etc. 
Lemma 10.2. $\mathcal{P}_{E}$ is a bijection, and its restriction to $\mathcal{H}_{E}^{-} \backslash \mathcal{P}_{E}^{-1}\left(\partial \mathcal{H}_{E}^{+}\right)$is smooth.

Proof. $\mathcal{P}_{E}$ is one-to-one: If $\mathcal{P}_{E}\left(x_{1}\right)=\mathcal{P}_{E}\left(x_{2}\right)$ and $T_{E}\left(x_{1}\right) \leq T_{E}\left(x_{2}\right)$, then $x_{1}=$ $\Phi^{t_{2}-t_{1}}\left(x_{2}\right)$ and

- either $x_{1} \in \partial \mathcal{D}_{E}^{-}$. Then $x_{2}=x_{1}$ since by the virial inequality

$$
\Phi^{t}\left(\mathcal{H}_{E}^{-}\right) \cap \partial \mathcal{D}_{E}^{-}=\emptyset \text { for } t>0,
$$

- or $x_{1} \in \mathcal{H}_{E}$. Then by definition of $T_{E}$ using the infimum, $x_{2}=x_{1}$, too.

$\mathcal{P}_{E}$ is onto: Time reversal

$$
\mathcal{T R}: P \rightarrow P, \quad(\vec{p}, \vec{q}) \in T^{*} \hat{M} \mapsto(-\vec{p}, \vec{q}), \quad x \in P \backslash T^{*} \hat{M} \mapsto x
$$

is a smooth anti-symplectic transformation, with

$$
\mathcal{T R} \circ \Phi^{t} \circ \mathcal{T} \mathcal{R}=\Phi^{-t} \quad(t \in \mathbb{R})
$$

and

$$
\mathcal{T R}\left(\mathcal{H}_{E}^{ \pm}\right)=\mathcal{H}_{E}^{\mp}
$$

Thus

$$
\mathcal{P}_{E}^{-1}(x)=\mathcal{T} \mathcal{R} \circ \mathcal{P}_{E} \circ \mathcal{T R}(x)
$$

Smoothness of $\mathcal{P}_{E} \uparrow_{U}$ for $U:=\mathcal{H}_{E}^{-} \backslash \mathcal{P}_{E}^{-1}\left(\partial \mathcal{H}_{E}^{+}\right)$follows from transversality of the codimension one $\partial$-manifold $U \subset \Sigma_{E}$ to the flow $\Phi^{t} \uparrow_{\Sigma_{E}}$, since $\mathcal{P}_{E} \uparrow_{U}$ maps to inner points of $\mathcal{H}_{E}^{+}$.

Symbolic dynamics

In order to use symbolic dynamics, we introduce symbol sequences

$$
\underline{k}=\left(k_{i}\right)_{i \in I} \in \mathcal{S}^{I}
$$

over the alphabet

$$
\mathcal{S}:=\{1, \ldots, n\}
$$

where

$$
I \equiv I_{l}^{r}:=\{i \in \mathbb{Z} \mid l \leq i \leq r\}
$$

for $l, r \in \mathbb{Z} \cup\{ \pm \infty\}$ is a (finite, half-infinite or bi-infinite) interval.

$\underline{k}$ is called admissible if $k_{i} \neq k_{i+1}$ for all $\{i, i+1\} \subset I$.

For $E>E_{\text {th }}$ and $\left(k_{0}, k_{1}\right)$ admissible we set

$$
V_{E}\left(k_{0}, k_{1}\right):=W_{E}\left(k_{0}, k_{1}\right):=\mathcal{H}_{E}^{k_{0}, k_{1}},
$$


and for $\left(k_{-m}, \ldots, k_{0}\right)$ admissible

$$
W_{E}\left(k_{-m}, \ldots, k_{0}\right):=W_{E}\left(k_{-1}, k_{0}\right) \cap \mathcal{P}_{E}\left(W_{E}\left(k_{-m}, \ldots, k_{-1}\right)\right),
$$

resp. for $\left(k_{0}, \ldots, k_{m}\right)$ admissible

$$
V_{E}\left(k_{0}, \ldots, k_{m}\right):=V_{E}\left(k_{0}, k_{1}\right) \cap \mathcal{P}_{E}^{-1}\left(V_{E}\left(k_{1}, \ldots, k_{m}\right)\right),
$$

$m \geq 2$. Then by Lemma 10.2 the iterated maps

$$
\begin{aligned}
& \mathcal{P}_{E}\left(k_{0}, \ldots, k_{m}\right): V_{E}\left(k_{0}, \ldots, k_{m}\right) \rightarrow W_{E}\left(k_{0}, \ldots, k_{m}\right) \\
& \mathcal{P}_{E}\left(k_{0}, \ldots, k_{m}\right):=\mathcal{P}_{E}^{m-1} \uparrow_{V_{E}\left(k_{0}, \ldots, k_{m}\right)}
\end{aligned}
$$

are diffeomorphisms (we will show in Proposition 11.5 that the sets $V_{E}(\underline{k})$ are non-empty). Again, $\bar{W}_{E}(\underline{k})$ and $\bar{V}_{E}(\underline{k})$ denote the closures of these sets.

We decompose the Poincaré map $\mathcal{P}_{E}\left(k_{-1}, k_{0}, k_{1}\right)$ in the form

$$
\begin{aligned}
\mathcal{P}_{E}\left(k_{-1}, k_{0}, k_{1}\right)(x) & =\mathcal{P}_{E}^{-}\left(k_{-1}, k_{0}\right) \circ \mathcal{P}_{E}^{\prime}\left(k_{-1}, k_{0}, k_{1}\right) \circ \mathcal{P}_{E}^{+}\left(k_{0}, k_{1}\right)(x) \\
& \equiv \mathcal{P}_{E}^{-} \circ \mathcal{P}_{E}^{\prime} \circ \mathcal{P}_{E}^{+}(x) \quad\left(x \in V_{E}\left(k_{-1}, k_{0}, k_{1}\right)\right)
\end{aligned}
$$

with the diffeomorphisms

$$
\begin{aligned}
\mathcal{P}_{E}^{-}: V_{E}\left(k_{-1}, k_{0}\right) & \rightarrow V_{E}^{\prime}\left(k_{-1}, k_{0}\right):=\mathcal{P}_{E}^{-}\left(V_{E}\left(k_{-1}, k_{0}\right)\right) \subset \partial \mathcal{Z}^{k_{-1}, k_{0}} \\
x & \mapsto \Phi\left(T_{E}^{k_{-1}, k_{0},+}(x), x\right) \\
\left(\mathcal{P}_{E}^{+}\right)^{-1}: W_{E}\left(k_{0}, k_{1}\right) & \rightarrow W_{E}^{\prime}\left(k_{0}, k_{1}\right):=\left(\mathcal{P}_{E}^{+}\right)^{-1}\left(W_{E}\left(k_{0}, k_{1}\right)\right) \subset \partial \mathcal{Z}^{k_{0}, k_{1}} \\
x & \mapsto \Phi\left(T_{E}^{k_{0}, k_{1},-}(x), x\right)
\end{aligned}
$$

and

$$
\mathcal{P}_{E}^{\prime}: V_{E}^{\prime}\left(k_{-1}, k_{0}, k_{1}\right) \rightarrow W_{E}^{\prime}\left(k_{-1}, k_{0}, k_{1}\right) \quad, \quad x \mapsto \Psi_{l}^{+}(x)
$$

with $\Psi_{l}^{+}$defined in (8.2)) for

$$
\begin{aligned}
V_{E}^{\prime}\left(k_{-1}, k_{0}, k_{1}\right) & :=\mathcal{P}_{E}^{-}\left(V_{E}\left(k_{-1}, k_{0}, k_{1}\right)\right) \subset V_{E}^{\prime}\left(k_{-1}, k_{0}\right), \\
W_{E}^{\prime}\left(k_{-1}, k_{0}, k_{1}\right) & :=\left(\mathcal{P}_{E}^{+}\right)^{-1}\left(W_{E}\left(k_{-1}, k_{0}, k_{1}\right)\right) \subset W_{E}^{\prime}\left(k_{0}, k_{1}\right) .
\end{aligned}
$$

$\mathcal{P}_{E}^{ \pm}$are considered as perturbations of Poincaré maps for the free flow, whereas Proposition 4.1 allows us to view $\mathcal{P}_{E}^{\prime}$ as a perturbation of Poincare maps for the Kepler flow. 


\section{Adapted coordinates}

We now define adapted coordinates in the Poincaré sections $\mathcal{H}_{E}$. This will simplify to introduce invariant cone fields later on.

We complement the unit vectors

$$
\hat{s}^{ \pm} \equiv \hat{s}^{ \pm}\left(k_{-1}, k_{0}, k_{1}\right) \quad \text { with } \quad \hat{s}^{ \pm}:=\hat{s}^{k_{ \pm 1}, k_{0}}
$$

pointing towards $\vec{s}_{k_{0}}$ by a unit vector

$$
\hat{t}^{ \pm} \equiv \hat{t}^{ \pm}\left(k_{-1}, k_{0}, k_{1}\right) \quad \text { with } \quad \hat{t}^{+}=\hat{t}^{-} \text {perpendicular to } \operatorname{span}\left(\hat{s}^{-}, \hat{s}^{+}\right)
$$

(by the NC condition 2.4 the span is one-dimensional iff $k_{1}=k_{-1}$ ), and set

$$
\hat{u}^{ \pm} \equiv \hat{u}^{ \pm}\left(k_{-1}, k_{0}, k_{1}\right):=\hat{s}^{ \pm} \times \hat{t}^{ \pm} .
$$

Then $\hat{t}^{ \pm}$and $\hat{u}^{ \pm}$, considered as elements of $T_{\vec{q}} \mathbb{R}_{\vec{q}}^{3}$ for $\vec{q} \in \overline{\mathcal{H}}_{M}^{k_{ \pm 1}, k_{0}}$, form an orthonormal basis of $T_{\vec{q}} \overline{\mathcal{H}}_{M}^{k_{ \pm 1}, k_{0}}$. We introduce adapted coordinates on $V_{E}\left(k_{-1}, k_{0}\right)$ and $W_{E}\left(k_{0}, k_{1}\right)$ by mapping

$$
\begin{aligned}
V_{E}\left(k_{-1}, k_{0}\right) \rightarrow \mathbb{R}^{2} \times \mathbb{R}^{2} \quad, \quad(\vec{p}, \vec{q}) \mapsto\left(\vec{y}^{-}, \vec{z}^{-}\right), \\
W_{E}\left(k_{0}, k_{1}\right) \rightarrow \mathbb{R}^{2} \times \mathbb{R}^{2} \quad, \quad(\vec{p}, \vec{q}) \mapsto\left(\vec{y}^{+}, \vec{z}^{+}\right),
\end{aligned}
$$

with

$$
\vec{y}^{ \pm}:=\left(\begin{array}{l}
\left\langle\vec{p}, \hat{t}^{ \pm}\right\rangle \\
\left\langle\vec{p}, \hat{u}^{ \pm}\right\rangle
\end{array}\right) / \sqrt{2 E} \quad, \quad \vec{z}^{ \pm}:=\left(\begin{array}{c}
\left\langle\vec{q}-\vec{m}^{k_{ \pm}, k_{0}}, \hat{t}^{ \pm}\right\rangle \\
\left\langle\vec{q}-\vec{m}^{k_{ \pm 1}, k_{0}}, \hat{u}^{ \pm}\right\rangle
\end{array}\right) / l^{ \pm}
$$

and $l^{ \pm}:=\left|\vec{m}^{k_{0, \pm 1}}-\vec{s}_{k_{0}}\right|=\frac{1}{2} d^{k_{ \pm 1}, k_{0}}$.

Remark 10.3. The coordinates $\left(\vec{y}^{-}, \vec{z}^{-}\right)$on $V_{E}\left(k_{-1}, k_{0}\right)$ depend through the vector $\hat{t}^{-} \equiv \hat{t}^{-}\left(k_{-1}, k_{0}, k_{1}\right)$ on the symbol $k_{1}$. However, the coordinate systems for the symbols $k_{1}^{I}$ and $k_{1}^{I I}$ are related by

$$
\left(\begin{array}{l}
\vec{y}^{-, I} \\
\vec{z}^{-, I}
\end{array}\right)=\left(\begin{array}{c}
O \vec{y}^{-, I I} \\
O \vec{z}^{-, I I}
\end{array}\right),
$$

where $O \in S O(2, \mathbb{R})$ is the rotation in the plane spanned by $\hat{t}^{-, I}$ and $\hat{u}^{-, I}$ which maps $\hat{t}^{-, I}$ onto $\hat{t}^{-, I I}$ (and correspondingly $\hat{u}^{-, I}$ onto $\hat{u}^{-, I I}$ ).

Similarly we introduce coordinates in

$$
\begin{array}{rlrl}
V_{E}^{\prime}\left(k_{-1}, k_{0}\right) & \rightarrow \mathbb{R}^{2} \times \mathbb{R}^{2}, & (\vec{p}, \vec{q}) \mapsto\left(\vec{y}^{\prime-}, \vec{z}^{\prime-}\right) \\
W_{E}^{\prime}\left(k_{0}, k_{1}\right) \rightarrow \mathbb{R}^{2} \times \mathbb{R}^{2}, & (\vec{p}, \vec{q}) \mapsto\left(\vec{y}^{\prime+}, \vec{z}^{\prime+}\right)
\end{array}
$$

with

$$
\begin{gathered}
\vec{y}^{\prime \pm}:=\left(\begin{array}{c}
\left\langle\vec{p}, \hat{t}^{ \pm}\right\rangle \\
\left\langle\vec{p}, \hat{u}^{ \pm}\right\rangle
\end{array}\right) / \sqrt{2 E} \quad, \quad \vec{z}^{\prime \pm}:=\left(\begin{array}{c}
\left\langle\vec{q}-\vec{n}^{k_{ \pm 1}, k_{0}}, \hat{t}^{ \pm}\right\rangle \\
\left\langle\vec{q}-\vec{n}^{k_{1}, k_{0}}, \hat{u}^{ \pm}\right\rangle
\end{array}\right) / l^{\prime \pm}, \quad \\
l^{\prime-}:=\left|\vec{n}^{k_{-1}, k_{0}}-\vec{s}_{k_{-1}}\right|=d^{k_{-1}, k_{0}}-c_{q} \quad \text { and } \quad l^{\prime+}:=\left|\vec{n}^{k_{1}, k_{0}}-\vec{s}_{k_{0}}\right|=c_{q} .
\end{gathered}
$$

The next lemma shows that arbitrary pairs $\vec{z}^{-}, \vec{z}^{+}$of points in the configuration space projections of the Poincaré surfaces are connected by a trajectory. 
Lemma 10.4. Choose a large enough constant $e_{0}$ in Definition (9.16) of the Poincaré surfaces. Then for $E_{\mathrm{th}} \equiv E_{\mathrm{th}}\left(e_{0}\right)$ large, all $E>E_{\mathrm{th}}$ and

$$
\vec{z}^{ \pm} \in \mathcal{H}_{M}^{k_{ \pm 1}, k_{0}}(E)
$$

there exists a $x^{-} \in V_{E}\left(k_{-1}, k_{0}, k_{1}\right)$ of the form

$$
x^{-}=\left(\vec{y}^{-}, \vec{z}^{-}\right) \text {with } x^{+}:=\mathcal{P}_{E}\left(x^{-}\right)=\left(\vec{y}^{+}, \vec{z}^{+}\right) .
$$

Proof. By (9.16) in the $\left(\vec{y}^{-}, \vec{z}^{-}\right)$-coordinates $V_{E}\left(k_{-1}, k_{0}\right)=\mathcal{H}_{E}^{k_{-1}, k_{0}}$ is of the form

$$
V_{E}\left(k_{-1}, k_{0}\right)=B_{y} \times B_{z}
$$

with the two-disks $B_{y}$ in momentum space and $B_{z}$ in configuration space

$$
B_{y}:=\left\{\vec{y} \in \mathbb{R}^{2}|| \vec{y} \mid<\frac{1}{2} c_{p} e_{0} / E\right\} \supset B_{z}:=\left\{\vec{z} \in \mathbb{R}^{2}|| \vec{z} \mid<\frac{1}{4} c_{p} e_{0} / E\right\} .
$$

We thus consider initial conditions of the form

$$
x^{-}=\left(\vec{y}^{-}, \vec{z}^{-}\right) \in B_{y} \times\left\{\vec{z}^{-}\right\} \subset V_{E}\left(k_{-1}, k_{0}\right)
$$

and their images

$$
x^{\prime-}=\left(\vec{y}^{\prime-}, \vec{z}^{\prime-}\right):=\mathcal{P}_{E}^{-}\left(x^{-}\right) \in V_{E}^{\prime}\left(k_{-1}, k_{0}\right) .
$$

The idea of the proof is that, as $B_{y}$ has twice the size of $B_{z}$, the family of trajectories with initial conditions in $B_{y} \times\left\{\vec{z}^{-}\right\}$has an opening angle $\mathcal{O}(1 / E)$ that is large enough to hit a full $\mathcal{O}(1 / E)$-neighbourhood of $c_{k_{0}}$. Then by near collision, the opening angle is nearly amplified to $2 \pi$, if $e_{0}$ is chosen large enough.

The threshold energy $E_{\mathrm{th}}$ depends on the constant $e_{0}$. However, by Lemma 7.1 and a compactness argument one sees that the error term $\mathcal{O}(1 / E)$ in the $C^{1}$-estimates

$$
\begin{aligned}
& \left|\left(\begin{array}{l}
\vec{y}^{\prime-} \\
\vec{z}^{\prime-}
\end{array}\right)-\left(\begin{array}{cc}
1 & 0 \\
\left(1-\frac{l^{-}}{l^{\prime}}\right) 11 & \frac{l^{-}}{l^{\prime}} \mathbb{1}
\end{array}\right)\left(\begin{array}{l}
\vec{y}^{-} \\
\vec{z}^{-}
\end{array}\right)\right| \leq C / E \\
& \left|\left(\begin{array}{c}
\vec{y}^{+} \\
\vec{z}^{+}
\end{array}\right)-\left(\begin{array}{cc}
1 & 0 \\
\left(1-\frac{l^{++}}{l^{+}}\right) 11 & \frac{l^{l^{+}}}{l^{+}}
\end{array}\right)\left(\begin{array}{l}
\vec{y}^{\prime+} \\
\vec{z}^{\prime+}
\end{array}\right)\right| \leq C / E
\end{aligned}
$$

and an identical estimate for the linearizations

$$
\begin{aligned}
& \left|\left(\begin{array}{l}
\delta \vec{y}^{\prime-} \\
\delta \vec{z}^{\prime-}
\end{array}\right)-\left(\begin{array}{cc}
1 & 0 \\
\left(1-\frac{l^{-}}{l^{\prime}}\right) 11 & \frac{l^{-}}{l^{\prime-}} 1
\end{array}\right)\left(\begin{array}{l}
\delta \vec{y}^{-} \\
\delta \vec{z}^{-}
\end{array}\right)\right| \leq \frac{C}{E} \cdot\left|\left(\begin{array}{l}
\delta \vec{y}^{-} \\
\delta \vec{z}^{-}
\end{array}\right)\right| \\
& \left|\left(\begin{array}{c}
\delta \vec{y}^{+} \\
\delta \vec{z}^{+}
\end{array}\right)-\left(\begin{array}{cc}
1 & 0 \\
\left(1-\frac{l^{\prime+}}{l^{+}}\right) 11 & \frac{l^{+}}{l^{+}} 1
\end{array}\right)\left(\begin{array}{l}
\delta \vec{y}^{\prime+} \\
\delta \vec{z}^{\prime+}
\end{array}\right)\right| \leq \frac{C}{E} \cdot\left|\left(\begin{array}{l}
\delta \vec{y}^{\prime+} \\
\delta \vec{z}^{\prime+}
\end{array}\right)\right|
\end{aligned}
$$

of these maps do not depend on $e_{0}$, that is, $C>0$ may be chosen fixed and then $e_{0}$ chosen arbitrarily large. 
The relations

$$
\begin{aligned}
& \vec{p}=\sqrt{2 E^{ \pm}} \cdot\left(\mp \hat{s}^{ \pm}+y_{1}^{ \pm} \hat{t}^{ \pm}+y_{2}^{ \pm} \hat{u}^{ \pm}+\mathcal{O}\left(E^{-2}\right)\right) \\
& \vec{q}=\vec{m}^{ \pm}+l^{ \pm}\left(z_{1}^{ \pm} \hat{t}^{ \pm}+z_{2}^{ \pm} \hat{u}^{ \pm}\right)
\end{aligned}
$$

with $E^{ \pm}:=E-V\left(\vec{m}^{ \pm}\right)$show that the angular momentum relative to the $k_{0}$-st nucleus before and after scattering equals

$\vec{L}_{k_{0}} \equiv\left(\vec{q}-\vec{s}_{k_{0}}\right) \times \vec{p}= \pm \sqrt{2 E^{ \pm}} l^{ \pm} \cdot\left(\left(y_{1}^{ \pm}+z_{1}^{ \pm}\right) \hat{u}^{ \pm}-\left(y_{2}^{ \pm}+z_{2}^{ \pm}\right) \hat{t}^{ \pm}+\mathcal{O}\left(E^{-2}\right)\right)$.

Thus $\left|\vec{L}_{k_{0}}\right|=\mathcal{O}\left(e_{0} / \sqrt{E}\right)$, and

$$
\left|\vec{L}_{k_{0}}\right| \geq \frac{1}{4} l^{ \pm} c_{p} e_{0} / \sqrt{E} \text { for }\left(\vec{y}^{-}, \vec{z}^{-}\right) \text {with } \vec{y}^{-} \in \partial \overline{B_{y}} .
$$

Estimate (10.17) shows that similar statements hold true for $\vec{L}_{k_{0}} \circ \mathcal{P}_{E}^{-}$.

Finally (changing to $(\vec{p}, \vec{q})$-coordinates for a moment) by Proposition 8.6 we know that the image

$$
x^{\prime+} \equiv\left(\vec{p}^{\prime+}, \vec{q}^{\prime+}\right):=\psi_{l}^{+}\left(x^{\prime-}\right) \in W_{E}^{\prime}\left(k_{-1}, k_{0}, k_{1}\right)
$$

of $x^{\prime-} \in V_{E}^{\prime}\left(k_{-1}, k_{0}, k_{1}\right)$ is near to the image

$$
x_{L}^{\prime+} \equiv\left(\vec{p}_{L}^{\prime+}, \vec{q}_{L}^{\prime+}\right):=\psi_{L, l}^{+}\left(x_{L}^{\prime-}\right) \in W_{E}^{\prime}\left(k_{-1}, k_{0}, k_{1}\right)
$$

of a point $x_{L}^{\prime-}=\Xi\left(x^{\prime-}\right)$ in a $\mathcal{O}(1 / E)-$ neighbourhood of $x^{\prime-}$ in the sense that

$$
\left|\vec{p}^{\prime+}-\vec{p}_{L}^{\prime+}\right| / \sqrt{2 E}+\left|\vec{q}^{\prime+}-\vec{q}_{L}^{\prime+}\right|=\mathcal{O}(1 / E) .
$$

For the Kepler exit map $\psi_{L, l}^{+}$the formula

$$
e \equiv e\left(x_{L}^{\prime-}\right)=\sqrt{1+2 E \vec{L}^{2} / Z^{2}}
$$

for the eccentricity of the Kepler hyperbola parametrized by

$$
r(\varphi)=\frac{\vec{L}^{2}}{Z(1+e \cos (\varphi))}
$$

shows that the maximal total scattering angle $2 \arccos (1 / e) \in[0, \pi)$ can be increased to $\pi-\varepsilon$ (uniformly in $E$ ) by increasing $e_{0}$, so that the diffeomorphism

$$
B_{y} \times\left\{\vec{z}^{-}\right\} \ni x^{-} \mapsto \vec{q}_{L}^{+}\left(\Xi \circ \mathcal{P}_{E}^{-}\left(x^{-}\right)\right) \in \partial B_{k_{0}}\left(c_{q}\right)
$$

onto its image covers the sphere $\partial B_{k_{0}}\left(c_{q}\right)$ around $\vec{s}_{k_{0}}$ except an $\varepsilon$-neighbourhood of the forward direction $\vec{s}_{k_{0}}+c_{q} \cdot \hat{s}^{-} \in \partial B_{k_{0}}\left(c_{q}\right)$.

The same holds true for the diffeomorphism

$$
B_{y} \times\left\{\vec{z}^{-}\right\} \ni x^{-} \mapsto \vec{q}^{\prime+}\left(\mathcal{P}_{E}^{-}\left(x^{-}\right)\right) \in \partial B_{k_{0}}\left(c_{q}\right)
$$

onto its image. So by the NC condition 2.4 an $\varepsilon$-neighbourhood $U \subset \partial B_{k_{0}}\left(c_{q}\right)$ of the point $\vec{n}^{k_{1}, k_{0}}=\vec{s}_{k_{0}}-c_{q} \hat{s}^{+}$is contained in that image for $e_{0}$ large. By Definition 
(10.14) of the $\vec{z}^{\prime+}$-coordinates this corresponds to a $\varepsilon$-neighbourhood of $\vec{z}^{\prime+}=0$. Estimate (9.22) then implies that on this neighbourhood

$$
\vec{y}^{\prime+}\left(\mathcal{P}_{E}^{\prime} \circ \mathcal{P}_{E}^{-}\left(x^{-}\right)\right)=\vec{z}^{\prime+}\left(\mathcal{P}_{E}^{\prime} \circ \mathcal{P}_{E}^{-}\left(x^{-}\right)\right)+\mathcal{O}(1 / E) \quad\left(x^{-} \in B_{y} \times\left\{\vec{z}^{-}\right\}\right) .
$$

Thus we conclude from (10.18) that the intersection

$$
\mathcal{P}_{E}\left(B_{y} \times\left\{\vec{z}^{-}\right\}\right) \equiv \mathcal{P}_{E}^{+} \circ \mathcal{P}_{E}^{\prime} \circ \mathcal{P}_{E}^{-}\left(B_{y} \times\left\{\vec{z}^{-}\right\}\right) \cap B_{y} \times\left\{\vec{z}^{+}\right\} \subset W\left(k_{0}, k_{1}\right)
$$

is non-empty.

Remark 10.5. In fact the point $x^{-} \in V_{E}\left(k_{-1}, k_{0}, k_{1}\right)$ is unique, as will follow from Proposition 11.2 below.

Lemma 10.6. For all admissible $\left(k_{-1}, k_{0}, k_{1}\right) \in \mathcal{S}^{3}$ the Poincaré return time equals

$T_{E}\left\lceil V_{E}\left(k_{-1}, k_{0}, k_{1}\right)=\frac{d^{k_{-1}, k_{0}}+d^{k_{0}, k_{1}}}{2 \sqrt{2 E}}-\frac{Z_{k_{0}}}{(2 E)^{3 / 2}} \ln \left(E \frac{d^{k_{-1}, k_{0}}+d^{k_{0}, k_{1}}}{2\left|Z_{k_{0}}\right|}\right)+\mathcal{O}\left(E^{-3 / 2}\right)\right.$.

Proof. $\quad T_{E}(x)=T_{E}^{k_{-1}, k_{0},+}(x)+\left(T_{k_{0}}^{+}(y)-T_{k_{0}}^{-}(y)\right)-T_{E}^{k_{0}, k_{1},-}\left(\mathcal{P}_{E}(x)\right)$

with $y:=\psi_{k_{0}}^{0} \circ \Phi\left(T^{k_{-1}, k_{0},+}(x), x\right)$. The estimate thus follows from (9.21), (8.16) and (8.18).

\section{Existence of an invariant cone field}

Definition 11.1. A cone at $x \in \mathcal{H}_{E}$ is the image of the standard cone

$$
\left\{(\vec{u}, \vec{v}) \in \mathbb{R}^{2} \times \mathbb{R}^{2}|| \vec{u}|\geq| \vec{v} \mid\right\}
$$

w.r.t. an invertible linear map

$$
\mathbb{R}^{2} \times \mathbb{R}^{2} \rightarrow T_{x} \mathcal{H}_{E}
$$

A cone field $\mathcal{C}$ in $U \subset \mathcal{H}_{E}$ associates to every $x \in U$ a cone $\mathcal{C}(x)$ at $x$. $\mathcal{C}$ is called invariant if for every $x \in U$ with $\mathcal{P}_{E}(x) \in U$

$$
T_{x} \mathcal{P}_{E}(\mathcal{C}(x)) \subset \mathcal{C}\left(\mathcal{P}_{E}(x)\right),
$$

and strictly invariant if

$$
T_{x} \mathcal{P}_{E}(\mathcal{C}(x)) \subset \operatorname{Int}\left(\mathcal{C}\left(\mathcal{P}_{E}(x)\right)\right) \cup\{0\}
$$

Alternatively one may think of cones as subsets of $\mathbb{R} P^{3}$.

In the next proposition we use the Poincaré section coordinates $\left(\vec{y}^{ \pm}, \vec{z}^{ \pm}\right)$ defined in (10.13). 
Proposition 11.2. For $C>0$ large and $E>E_{\text {th }}$ the cone field $\mathcal{C}$ in

$$
\begin{gathered}
U:=\bigcup_{\substack{\left(k_{-1}, k_{0}, k_{1}\right) \in \mathcal{S}^{3} \\
\text { admissible }}} V_{E}\left(k_{-1}, k_{0}, k_{1}\right) \\
\mathcal{C}(x):=\left\{(\delta \vec{y}, \delta \vec{z}) \in T_{(\vec{y}, \vec{z})} V_{E}\left(k_{-1}, k_{0}, k_{1}\right)|| \delta \vec{y}-\delta \vec{z}\left|\leq \frac{C}{E}\right| \delta \vec{y}+\delta \vec{z} \mid\right\}
\end{gathered}
$$

for $x \equiv(\vec{y}, \vec{z}) \in U$, is strictly $\mathcal{P}_{E}$-invariant, and the linearized Poincaré map equals

$$
T_{x} \mathcal{P}_{E}=f\left(k_{-1}, k_{0}, k_{1}\right) E \cdot\left(\begin{array}{ll}
1 & 1 \\
1 & 11
\end{array}\right)+\mathcal{O}\left(E^{0}\right) \quad\left(x \in V_{E}\left(k_{-1}, k_{0}, k_{1}\right)\right),
$$

with

$$
f\left(k_{-1}, k_{0}, k_{1}\right):=\frac{2 d^{k_{-1}, k_{0}} \cos ^{2}\left(\frac{1}{2} \alpha\left(k_{-1}, k_{0}, k_{1}\right)\right)}{-Z_{k_{0}}} .
$$

Remarks 11.3. 1) The linearized Poincare map (11.2) is invertible, although the matrix $\left(\begin{array}{ll}11 & \mathbb{1} \\ \mathbb{1 1} & \mathbb{1}\end{array}\right)$ is not.

2) Although the coordinates $\left(\vec{y}^{+}, \vec{z}^{+}\right)$on $W_{E}\left(k_{-1}, k_{0}, k_{1}\right) \subset \mathcal{H}_{E}^{k_{0}, k_{1}}$ do not coincide on their common domain $W_{E}\left(k_{-1}, k_{0}, k_{1}\right) \cap V_{E}\left(k_{0}, k_{1}, k_{2}\right)$ with the coordinates $\left(\vec{y}^{-}, \vec{z}^{-}\right)$on $V_{E}\left(k_{0}, k_{1}, k_{2}\right) \subset \mathcal{H}_{E}^{k_{0}, k_{1}}$, they are related by

$$
\left(\begin{array}{l}
\vec{y}^{+} \\
\vec{z}^{+}
\end{array}\right)=\left(\begin{array}{c}
O \vec{y}^{-} \\
O \vec{z}^{-}
\end{array}\right)
$$

where $O \in O(2, \mathbb{R})$ is the reflection in the plane perpendicular to $\hat{s}^{k_{0}, k_{1}}$, transforming the unit vector $\hat{t}^{+}\left(k_{-1}, k_{0}, k_{1}\right)$ into $\hat{t}^{-}\left(k_{0}, k_{1}, k_{2}\right)$ and the unit vector $\hat{u}^{+}\left(k_{-1}, k_{0}, k_{1}\right)$ into $\hat{u}^{-}\left(k_{0}, k_{1}, k_{2}\right)$, see Definition (10.11) and (10.12). Thus the above cone field $\mathcal{C}$ is invariant under that transformation.

Proof. By (10.19)

$$
\begin{aligned}
\left(\begin{array}{l}
\delta \vec{y}^{\prime-} \\
\delta \vec{z}^{\prime-}
\end{array}\right) & :=\left(T_{x^{-}} \mathcal{P}_{E}^{-}\right)\left(\begin{array}{c}
\delta \vec{y}^{-} \\
\delta \vec{z}^{-}
\end{array}\right) \\
& =\left(\begin{array}{cc}
1 & 0 \\
\left(1-\frac{l^{-}}{l^{\prime}}\right) \mathbb{1} & \frac{l^{-}}{l^{\prime-}} \mathbb{1}
\end{array}\right)\left(\begin{array}{l}
\delta \vec{y}^{-} \\
\delta \vec{z}^{-}
\end{array}\right)+\left|\left(\begin{array}{l}
\delta \vec{y}^{-} \\
\delta \vec{z}^{-}
\end{array}\right)\right| \cdot \mathcal{O}(1 / E) .
\end{aligned}
$$

From (8.3) and (10.14) we deduce the transformation formulae

$$
\left(\begin{array}{l}
\left\langle\delta \vec{v}^{-}, \hat{t}^{-}\right\rangle \\
\left\langle\delta \vec{v}^{-}, \hat{u}^{-}\right\rangle
\end{array}\right)=\delta \vec{y}^{\prime-} \quad \text { and } \quad\left(\begin{array}{l}
\left\langle\delta \vec{w}^{-}, \hat{t}^{-}\right\rangle \\
\left\langle\delta \vec{w}^{-}, \hat{u}^{-}\right\rangle
\end{array}\right)=\left(l^{\prime-} / c_{q}\right) \delta \vec{z}^{\prime-}
$$

from the $\left(\delta \vec{y}^{\prime-}, \delta \vec{z}^{\prime-}\right)$-coordinates to the $\left(\delta \vec{v}^{-}, \delta \vec{w}^{-}\right)$-coordinates. 
After near-collision we have

$$
\left(\begin{array}{c}
\left\langle\delta \vec{v}^{+}, \hat{t}^{+}\right\rangle \\
\left\langle\delta \vec{v}^{+}, \hat{u}^{+}\right\rangle
\end{array}\right)=\delta \vec{y}^{\prime+} \text { and } \quad\left(\begin{array}{c}
\left\langle\delta \vec{w}^{+}, \hat{t}^{+}\right\rangle \\
\left\langle\delta \vec{w}^{+}, \hat{u}^{+}\right\rangle
\end{array}\right)=\delta \vec{z}^{\prime+} .
$$

The reflection $R_{\hat{s}^{-}-\hat{s}^{+}}$by the plane perpendicular to the vector $\hat{s}^{-}-\hat{s}^{+}$transforms the unit vectors as follows:

$$
\hat{s}^{+}=-R_{\hat{s}^{+}-\hat{s}^{-}}\left(\hat{s}^{-}\right) \quad, \quad \hat{t}^{+}=+R_{\hat{s}^{+}-\hat{s}^{-}}\left(\hat{t}^{-}\right) \quad \text { and } \quad \hat{u}^{+}=+R_{\hat{s}^{+}-\hat{s}^{-}}\left(\hat{u}^{-}\right) .
$$

Moreover, up to an error term $\mathcal{O}(1 / E)$, we may substitute $R_{\hat{S}^{+}-\hat{s}^{-}}$for the reflection $R_{\vec{v}^{+}-\vec{v}^{-}}$in (8.9), since by estimate (9.22) the differences $\left|\vec{v}^{ \pm}-\hat{s}^{ \pm}\right|=\mathcal{O}(1 / E)$ (and since $\left|\hat{s}^{+}-\hat{s}^{-}\right|>0$ ). In particular

$$
\Delta \psi=\pi-\alpha\left(k_{-1}, k_{0}, k_{1}\right)+\mathcal{O}(1 / E) .
$$

Thus putting together (11.4), (11.5), (8.9), (8.21), (11.6) and (11.7), we obtain

$$
\begin{aligned}
&\left(\begin{array}{l}
\delta \vec{y}^{\prime+} \\
\delta \vec{z}^{\prime+}
\end{array}\right)= \frac{4 c_{q} \sin ^{2}\left(\frac{1}{2} \Delta \psi\right) E}{-Z_{k_{0}}} . \\
&\left(\begin{array}{ll}
11 & 11 \\
11 & 11
\end{array}\right)\left(\begin{array}{ll}
11 & 0 \\
0 & \left(l^{-} / c_{q}\right) 1
\end{array}\right)\left(\begin{array}{cc}
1 & 0 \\
\left(1-\frac{l^{-}}{l^{\prime}}\right) 11 & \frac{l^{-}}{l^{\prime-}} \mathbb{1}
\end{array}\right)\left(\begin{array}{l}
\delta \vec{y}^{-} \\
\delta \vec{z}^{-}
\end{array}\right) \\
&+\left|\left(\begin{array}{l}
\delta \vec{y}^{-} \\
\delta \vec{z}^{-}
\end{array}\right)\right| \cdot \mathcal{O}\left(E^{0}\right) \\
&=
\end{aligned}
$$

as $c_{q}+l^{--}-l^{-}=l^{-}$. Inserting (11.8) in (10.20) yields

$$
\begin{aligned}
\left(\begin{array}{c}
\delta \vec{y}^{+} \\
\delta \vec{z}^{+}
\end{array}\right) & =\left(\begin{array}{cc}
1 & 0 \\
\left(1-\frac{c_{q}}{l^{+}}\right) \mathbb{1 1} & \frac{c_{q}}{l^{+}} \mathbb{1}
\end{array}\right)\left(\begin{array}{l}
\vec{y}^{\prime+} \\
\vec{z}^{\prime+}
\end{array}\right)+\left|\left(\begin{array}{c}
\delta \vec{y}^{\prime+} \\
\delta \vec{z}^{\prime+}
\end{array}\right)\right| \cdot \mathcal{O}(1 / E) \\
& =f\left(k_{-1}, k_{0}, k_{1}\right) E\left(\begin{array}{ll}
11 & 11 \\
11 & 11
\end{array}\right)\left(\begin{array}{l}
\delta \vec{y}^{-} \\
\delta \vec{z}^{-}
\end{array}\right)+\left|\left(\begin{array}{c}
\delta \vec{y}^{-} \\
\delta \vec{z}^{-}
\end{array}\right)\right| \cdot \mathcal{O}\left(E^{0}\right),
\end{aligned}
$$

as $l^{-}=\frac{1}{2} d^{k_{-1}, k_{0}}$, proving (11.2).

Strict invariance of the cone field $\mathcal{C}$ then follows from (11.2).

We now describe the domains $V_{E}(\underline{k})$ and images $W_{E}(\underline{k})$ of the iterated Poincaré map with more precision. So let

$$
V_{E}(\underline{k})(\vec{z}):=V_{E}(\underline{k}) \cap\left(B_{y} \times\{\vec{z}\}\right) \quad\left(\vec{z} \in B_{z}\right)
$$

and similarly

$$
W_{E}(\underline{k})(\vec{z}):=W_{E}(\underline{k}) \cap\left(B_{y} \times\{\vec{z}\}\right) \quad\left(\vec{z} \in B_{z}\right)
$$


consist of the points with the same configuration space coordinate $\vec{z}$. We also consider them as subsets of $B_{y}$, forgetting the fixed coordinate $\vec{z}$. It turns out that they are diffeomorphic to two-dimensional disks. We call

$$
\operatorname{diam}_{y}\left(V_{E}(\underline{k})\right):=\sup _{\vec{z} \in B_{z}} \operatorname{diam}\left(V_{E}(\underline{k})(\vec{z})\right),
$$

resp.

$$
\operatorname{diam}_{y}\left(W_{E}(\underline{k})\right):=\sup _{\vec{z} \in B_{z}} \operatorname{diam}\left(W_{E}(\underline{k})(\vec{z})\right)
$$

the $y$-diameter of $V_{E}(\underline{k})$, resp. $W_{E}(\underline{k})$ (which is measured with the Euclidean metric in the $\vec{y}$ coordinates).

Corollary 11.4. For all $\vec{z}^{-} \in B_{z}$ the map

$$
V_{E}\left(k_{-1}, k_{0}, k_{1}\right)\left(\vec{z}^{-}\right) \ni \vec{y}^{-} \mapsto \vec{z}^{+}\left(\vec{y}^{-}, \vec{z}^{-}\right) \in B_{z}
$$

is a diffeomorphism. Thus the domain can be represented as

$$
V_{E}\left(k_{-1}, k_{0}, k_{1}\right)\left(\vec{z}^{-}\right)=\left\{\vec{y}^{-}\left(\vec{z}^{-}, \vec{z}^{+}\right) \mid \vec{z}^{+} \in B_{z}\right\},
$$

with

$$
\begin{gathered}
D_{1} \vec{y}^{-}\left(\vec{z}^{-}, \vec{z}^{+}\right)=-11+\mathcal{O}\left(E^{-1}\right), \\
D_{2} \vec{y}^{-}\left(\vec{z}^{-}, \vec{z}^{+}\right)=\frac{1}{f\left(k_{-1}, k_{0}, k_{1}\right) E}+\mathcal{O}\left(E^{-2}\right),
\end{gathered}
$$

and

$$
\vec{y}^{-}\left(\vec{z}^{-}, \vec{z}^{+}\right)=-\vec{z}^{-}+\mathcal{O}\left(E^{-1}\right),
$$

the error terms being independent of the parameter $e_{0}$ in (9.16).

In particular the $y$-diameter of $V_{E}\left(k_{-1}, k_{0}, k_{1}\right)$ is of $\operatorname{order} \mathcal{O}\left(e_{0} / E^{2}\right)$.

Proof. From formula (11.2) for the derivative of the Poincaré map we see (observing that $f\left(k_{-1}, k_{0}, k_{1}\right) \neq 0$ ) that (11.10) is a local diffeomorphism, and in fact a diffeomorphism onto its image.

Thus by the definitions (10.9) and (10.8) of domain and image (11.10) is a diffeo. Estimates (11.11) and (11.12) follow by inverting (11.2). Defs. (9.16), (10.7) and (10.13) show that the $y$-diameter of $W_{E}\left(k_{0}, k_{1}\right)$ is of order $\mathcal{O}\left(e_{0} / E\right)$. Thus (11.12) implies that the $y$-diameter of $V_{E}\left(k_{-1}, k_{0}, k_{1}\right)$ is smaller by one order.

By definition (9.16), the domain $\mathcal{H}_{E}^{k, l}$ has size $\mathcal{O}\left(e_{0} / E\right)$, so that directly we get only $\vec{y}^{-}\left(\vec{z}^{-}, \vec{z}^{+}\right)=-\vec{z}^{-}+\mathcal{O}\left(e_{0} / E\right)$ instead of (11.13). However, we then see from (11.11) that we may enlarge the size parameter $e_{0}$ without enlarging the error, and thus prove (11.11).

Proposition 11.5. There exist $C_{6}>1$ and $\delta E>0$, such that for all $E>E_{\mathrm{th}}$, $m \geq 1, \underline{k} \in \mathbf{X}_{0}^{m}$ and $\left(\vec{y}_{\underline{k}}^{+}, \vec{z}_{\underline{k}}^{+}\right):=\overline{\mathcal{P}}_{E}(\underline{k})\left(\vec{y}^{-}, \vec{z}^{-}\right) \subset \overline{\mathcal{H}}_{E}^{k_{m-1}, k_{m}}$ the following holds true: 
1. For $V_{E}(\underline{k})\left(\vec{z}^{-}\right)$defined in (11.9) the maps

$$
V_{E}(\underline{k})\left(\vec{z}^{-}\right) \ni \vec{y}^{-} \mapsto \vec{z}_{\underline{k}}^{+}\left(\vec{y}^{-}, \vec{z}^{-}\right) \in B_{z} \quad\left(\vec{z}^{-} \in B_{z}\right)
$$

are diffeomorphisms.

2.

$$
D_{1} \vec{y}_{\underline{\underline{k}}}^{-}\left(\vec{z}^{-}, \vec{z}^{+}\right)=-11+\mathcal{O}\left(E^{-1}\right) \quad\left(\vec{z}^{ \pm} \in \bar{B}_{z}\right)
$$

uniformly in $m$.

3. For all $\vec{z}^{+} \in \bar{B}_{z}$ the vector field on $\bar{B}_{z}$ given by $\vec{z}^{-} \mapsto \vec{y}_{\underline{k}}^{-}\left(\vec{z}^{-}, \vec{z}^{+}\right)$points inside the boundary:

$$
\vec{y}_{\underline{k}}^{-}\left(\vec{z}^{-}, \vec{z}^{+}\right) \cdot \vec{z}^{-}<0 \quad\left(\vec{z}^{-} \in \partial \bar{B}_{z}\right) .
$$

4. $V_{E}(\underline{k})\left(\vec{z}^{-}\right)$contains a disk of radius

$$
C_{6}^{-1} E^{-1} \prod_{i=1}^{m-1}\left(\left|f\left(k_{i-1}, k_{i}, k_{i+1}\right)\right| \cdot(E+\delta E)\right)^{-1},
$$

and is contained in a disk of radius

$$
C_{6} E^{-1} \prod_{i=1}^{m-1}\left(\left|f\left(k_{i-1}, k_{i}, k_{i+1}\right)\right| \cdot(E-\delta E)\right)^{-1} .
$$

5. For $\underline{k}, \underline{l} \in \mathbf{X}_{0}^{m+1}$ with $k_{i}=l_{i}$ for $i=0, \ldots$, m but $k_{m+1} \neq l_{m+1}$

$$
\left.\left.\operatorname{dist}\left(V_{E}(\underline{k})\right), V_{E}(\underline{l})\right)\right) \geq C_{6}^{-1} E^{-1} \prod_{i=1}^{m-1}\left(\left|f\left(k_{i-1}, k_{i}, k_{i+1}\right)\right| \cdot(E+\delta E)\right)^{-1} \text {. }
$$

Remark 11.6. Using time reversal, one obtains similar estimates for $W_{E}(\underline{k})$.

Proof.

- $m=1$ :

Then $V_{E}\left(k_{0}, k_{1}\right)=B_{y} \times B_{z}$ by (10.15), and the radius of these two disks is proportional tp $1 / E$, see def. (10.16). So

$$
V_{E}\left(k_{0}, k_{1}\right)(\vec{z})=V_{E}\left(k_{0}, k_{1}\right) \cap\left(B_{y} \times\{\vec{z}\}\right)=B_{y} \times\{\vec{z}\},
$$

showing (11.17) and (11.18).

If $\underline{k}=\left(k_{0}, k_{1}, k_{2}\right)$ and $\underline{l}=\left(k_{0}, k_{1}, l_{2}\right)$ with $l_{2} \neq k_{2}$, then $V_{E}(\underline{k})$ and $V_{E}(\underline{l})$ are both contained in the Poincaré section $V_{E}\left(k_{0}, k_{1}\right)=\mathcal{H}_{E}^{k_{0}, k_{1}}$, but are mapped to different sections in the next iteration. These have a distance from each other that is bounded below by an energy-independent constant. On the other hand by (8.9), scattering by the nucleus $k_{1}$ only leads to an expansion of order $E$, which conversely implies

$$
\operatorname{dist}\left(V_{E}\left(k_{0}, k_{1}, k_{2}\right), V_{E}\left(k_{0}, k_{1}, l_{2}\right)\right) \geq C_{6}^{-1} E^{-1} .
$$


- $m \mapsto m+1$ :

By def. (10.9) of $V_{E}(\underline{k})$

$$
V_{E}\left(k_{0}, \ldots, k_{m}\right)(\vec{z})=\left(B_{y} \times\{\vec{z}\}\right) \cap \mathcal{P}_{E}^{-1}\left(V_{E}\left(k_{1}, \ldots, k_{m}\right)\right) .
$$

Thus the induction step for (11.17) and (11.18) is provided by

$$
D_{2} \vec{y}\left(\vec{z}, \vec{z}^{+}\right)=\frac{1}{f\left(k_{0}, k_{1}, k_{2}\right) E}+\mathcal{O}\left(E^{-2}\right) \quad\left(\vec{z} \in B_{z}\right),
$$

i.e. formula (11.12).

The additional control of the $\vec{z}$-dependence of the sets $V_{E}(\underline{k})$ needed for the distance estimate (11.19) is guaranteed by the coupling (11.13) between the $\vec{y}$ and the $\vec{z}$-coordinate.

- One application of the linearized Poincaré map (11.2) maps the cone field with cones

$$
\left\{(\delta \vec{y}, \delta \vec{z}) \in T_{(\vec{y}, \vec{z})} V_{E}\left(k_{-1}, k_{0}, k_{1}\right) \mid \delta \vec{y} \cdot \delta \vec{z} \geq 0\right\}
$$

into the invariant cone field (11.1). In particular this applies to vectors of the form $(\delta \vec{z}, 0)$.

The image of the invariant cone field $\mathcal{C}$ under time reversal (using $\mathcal{T R}$ defined in (10.5)) meets the equation

$$
|\delta \vec{y}+\delta \vec{z}| \leq \frac{C}{E}|\delta \vec{y}-\delta \vec{z}| .
$$

This then implies the uniform estimate (11.15).

- By (11.13)

$$
\operatorname{diam}_{y}\left(V_{E}\left(k_{0}, k_{1}, k_{2}\right)(\overrightarrow{0})=\mathcal{O}(1 / E) \quad \text { independent of } e_{0} .\right.
$$

Thus $\vec{y}^{-}\left(0, \vec{z}^{+}\right)=\mathcal{O}(1 / E)$ independent of $e_{0}$, too. So the estimate (11.15) of the first partial derivative gives.

$$
\vec{y}_{\underline{k}}^{-}\left(\vec{z}^{-}, \vec{z}^{+}\right)=\vec{y}_{\underline{k}}^{-}\left(\overrightarrow{0}, \vec{z}^{+}\right)+\int_{0}^{1} D_{1} \vec{y}_{\underline{k}}^{-}\left(t \vec{z}^{-}, \vec{z}^{+}\right) d t=-\vec{z}^{-}+\mathcal{O}(1 / E) .
$$

Now the modulus of $\vec{z}^{+} \in \partial \bar{B}_{z}$ equals $\frac{1}{4} c_{p} e_{0} / E$ (see def. (10.16)), or

$$
\vec{y}_{\underline{k}}^{-}\left(\vec{z}^{-}, \vec{z}^{+}\right) \cdot \vec{z}=\frac{1}{4} c_{p} e_{0}\left(-\frac{1}{4} c_{p} e_{0}+\mathcal{O}(1)\right) / E^{2} \quad\left(\vec{z}^{-} \in \partial \bar{B}_{z}\right) .
$$

Enlarging the parameter $e_{0}$ if necessary gives (11.16).

- To prove the iteration step for the first statement, we first show that that map (11.14) is onto:

For all $\vec{z}, \vec{z}^{-} \in B_{z}$ there exists an $\vec{y}^{-}$with $\vec{z}_{\underline{k}}^{+}\left(\vec{y}^{-}, \vec{z}^{-}\right)=\vec{z}$.

Namely for $\vec{z}^{+} \in B_{z}, \underline{k}^{I}:=\left(k_{0}, k_{1}, k_{2}\right)$ and $\underline{k}^{I I}:=\left(k_{1}, \ldots, k_{m}\right)$ we consider the vector field

$$
\vec{F}: B_{z} \rightarrow \mathbb{R}^{2} \quad, \quad \vec{F}(\vec{z}):=\vec{y}_{\underline{k}^{I I}}^{-}\left(\vec{z}, \vec{z}^{+}\right)-\vec{y}_{\underline{k}^{I}}^{+}\left(\vec{z}^{-}, \vec{z}\right) .
$$


If $\vec{F}$ has a zero, there exists an initial point $\left(\vec{y}^{-}, \vec{z}^{-}\right) \in V_{E}(\underline{k})\left(\vec{z}^{-}\right)$meeting (11.20). However, the vector field $\vec{z} \mapsto \vec{y}_{\underline{k}^{I I}}^{-}\left(\vec{z}, \vec{z}^{+}\right)$points inside the boundary of $\bar{B}_{z}$, whereas by time reversal

$$
\vec{y}_{\underline{k}^{I}}^{+}\left(\vec{z}^{-}, \vec{z}\right) \cdot \vec{z}>0 \quad\left(\vec{z} \in \partial \bar{B}_{z}\right)
$$

Thus $\vec{F}$ points inwards, too, so that the degree of the vector field $\vec{F}$ on the disk is non-zero, which in turn implies that $\vec{F}(\vec{z})=\overrightarrow{0}$ for some $\vec{z}$ (see, e.g., Hirsch [Hi], Chapter 5).

Inspection of the derivative (11.2) of the Poincare map shows that $\vec{y}^{-} \mapsto$ $\vec{z}_{\underline{k}}^{+}\left(\vec{y}^{-}, \vec{z}^{-}\right)$is injective, smooth and smoothly invertible.

Lemma 11.7. For $E>E_{\mathrm{th}}, l<0<r$ and $\underline{k}=\left(k_{l}, \ldots, k_{r}\right)$ admissible

$$
\begin{aligned}
& \operatorname{diam}\left(W_{E}\left(k_{l}, \ldots, k_{1}\right) \cap V_{E}\left(k_{0}, \ldots, k_{r}\right)\right) \leq \\
& \quad 4\left(\operatorname{diam}_{y}\left(W_{E}\left(k_{l}, \ldots, k_{1}\right)\right)+\operatorname{diam}_{y}\left(V_{E}\left(k_{0}, \ldots, k_{r}\right)\right)\right) .
\end{aligned}
$$

Proof. Set $\underline{l}:=\left(k_{l}, \ldots, k_{1}\right)$ and $\underline{m}:=\left(k_{0}, \ldots, k_{r}\right)$. Let $A^{I} \equiv\left(\vec{y}^{I}, \vec{z}^{I}\right)$ and $A^{I I} \equiv\left(\vec{y}^{I I}, \vec{z}^{I I}\right)$ be two points in $\bar{W}_{E}(\underline{l}) \cap V_{E}(\underline{m})$ and

$$
\vec{z}^{I,+}:=\vec{z}_{\underline{m}}^{+}\left(A^{I}\right) \in B_{z} \quad, \quad \vec{z}^{I I,-}:=\vec{z}_{\underline{l}}^{-}\left(A^{I I}\right)
$$

the $\vec{z}$-components of $\mathcal{P}_{E}(\underline{m})\left(A^{I}\right)$ resp. $\mathcal{P}_{E}(\underline{l})^{-1}\left(A^{I I}\right)$. The disks

$$
D^{I}:=\left\{x \in V_{E}(\underline{m}) \cap W_{E}(\underline{l}) \mid \vec{z}_{\underline{m}}^{+}(x)=\vec{z}^{I,+}\right\}
$$

and

$$
D^{I I}:=\left\{x \in V_{E}(\underline{m}) \cap W_{E}(\underline{l}) \mid \vec{z}_{\underline{\underline{l}}}^{-}(x)=\vec{z}^{I I,-}\right\}
$$

intersect in a (unique) point $A^{I I I} \equiv\left(\vec{y}^{I I I}, \vec{z}^{I I I}\right)$ of $\mathcal{H}_{E}^{k_{0}, k_{1}}$.

As $\left\|A^{I I}-A^{I}\right\| \leq\left\|A^{I I}-A^{I I I}\right\|+\left\|A^{I I I}-A^{I}\right\|$, the lemma follows from the estimates

$$
\operatorname{diam}\left(D^{I}\right) \leq 4 \operatorname{diam}_{y}\left(W_{E}(\underline{l})\right) \quad \text { and } \quad \operatorname{diam}\left(D^{I I}\right) \leq 4 \operatorname{diam}_{y}\left(V_{E}(\underline{m})\right),
$$

and by time reversal symmetry (10.5) it suffices to prove the first one.

$D^{I}$ is mapped diffeomorphically onto $W_{E}(\underline{l})\left(\vec{z}^{I}\right)$ by

$$
x \mapsto\left(\vec{y}^{\prime}, \vec{z}^{I}\right) \quad \text { with the unique } \quad \vec{y}^{\prime} \in B_{y} \text { meeting } \vec{z}_{\underline{\underline{l}}}^{-}\left(\vec{y}^{\prime}, \vec{z}^{I}\right)=\vec{z}_{\underline{l}}^{-}(x)
$$

(uniqueness of $\vec{y}^{\prime}$ follows from Proposition 11.5.1). By the estimate (11.2) on the linearized Poincaré map this map increases distances by a factor less than 2 . 


\section{Symbolic dynamics}

In this section we analyze the set

$$
b_{E}=b \cap \Sigma_{E}
$$

of bounded $\Phi^{t}$-orbits of energy $E$.

To that aim we equip the alphabet $\mathcal{S}=\{1, \ldots, n\}$ with the discrete topology, and for an interval (see (10.6)) $I \equiv I_{l}^{r} \subset \mathbb{Z}$ the space $\mathcal{S}^{I}$ with the product topology. Finally, we introduce the topological subspace

$$
\mathbf{X}_{l}^{r}:=\left\{\underline{k} \in \mathcal{S}^{I} \mid \underline{k} \text { admissible }\right\}
$$

of admissible sequences (that is, $k_{i+1} \neq k_{i}$ ), and use the abbreviations

$$
\mathbf{X}:=\mathbf{X}_{-\infty}^{\infty} \quad, \quad \mathbf{X}^{+}:=\mathbf{X}_{0}^{\infty} \quad \text { and } \quad \mathbf{X}^{-}:=\mathbf{X}_{-\infty}^{1} .
$$

The space $\mathbf{X}$ of bi-infinite admissible sequences is empty for $n=1$, consists of two points for $n=2$, and is a Cantor set for $n \geq 3$. From now on we assume $n \geq 2$.

The shift

$$
\sigma: \mathbf{X} \rightarrow \mathbf{X} \quad, \quad \sigma(\underline{k})_{i}:=k_{i+1} \quad(i \in \mathbb{Z})
$$

is a homeomorphism on $\mathbf{X}$. It is well-known that the topology on $\mathbf{X}$ is generated by the metric

$$
d(\underline{k}, \underline{l}):=\sum_{i \in \mathbb{Z}} 2^{-|i|} \cdot\left(1-\delta_{k_{i}, l_{i}}\right), \quad(\underline{k}, \underline{l} \in \mathbf{X}) .
$$

For an admissible sequence $\underline{k}^{+}=\left(k_{0}, k_{1}, \ldots\right) \in \mathbf{X}^{+}$we define

$$
V_{E}\left(\underline{k}^{+}\right):=\bigcap_{m \in \mathbb{N}} V_{E}\left(k_{0}, \ldots, k_{m}\right) \subset V_{E}\left(k_{0}, k_{1}\right) .
$$

Similarly, for an admissible sequence $\underline{k}^{-}=\left(\ldots, k_{0}, k_{1}\right) \in \mathbf{X}^{-}$we define

$$
W_{E}\left(\underline{k}^{-}\right):=\bigcap_{m \in \mathbb{N}} W_{E}\left(k_{-m}, \ldots, k_{1}\right) \subset W_{E}\left(k_{0}, k_{1}\right) .
$$

Lemma 12.1. For $E>E_{\mathrm{th}}$ and $\underline{k} \in \mathbf{X}^{+}$the sets $V_{E}\left(\underline{k}^{+}\right)$and $W_{E}\left(\underline{k}^{-}\right)$are the graphs of functions

$$
v_{E}\left(\underline{k}^{+}\right): B_{z} \rightarrow B_{y} \quad \text { resp. } \quad w_{E}\left(\underline{k}^{-}\right): B_{z} \rightarrow B_{y}
$$

meeting the Lipschitz estimates

$$
\left|v_{E}\left(\underline{k}^{+}\right)\left(\vec{z}_{1}\right)-v_{E}\left(\underline{k}^{+}\right)\left(\vec{z}_{2}\right)-\left(\vec{z}_{2}-\vec{z}_{1}\right)\right| \leq C \frac{\left|\vec{z}_{1}-\vec{z}_{2}\right|}{E} \quad\left(\vec{z}_{1}, \vec{z}_{2} \in B_{z}\right)
$$

resp.

$$
\left|w_{E}\left(\underline{k}^{-}\right)\left(\vec{z}_{1}\right)-w_{E}\left(\underline{k}^{-}\right)\left(\vec{z}_{2}\right)-\left(\vec{z}_{1}-\vec{z}_{2}\right)\right| \leq C \frac{\left|\vec{z}_{1}-\vec{z}_{2}\right|}{E} \quad\left(\vec{z}_{1}, \vec{z}_{2} \in B_{z}\right)
$$

for some $C \equiv C\left(E_{\mathrm{th}}\right)$. 
Proof. The sets in (12.3) are nested: $V_{E}\left(k_{0}, \ldots, k_{m_{2}}\right) \subset V_{E}\left(k_{0}, \ldots, k_{m_{1}}\right)$ for $m_{2} \geq m_{1}$. By estimate (11.18), the $y$-diameter of these sets goes to zero as $m \rightarrow \infty$.

We set $\underline{l}:=\left(k_{0}, \ldots, k_{m}\right)$. For $\vec{z}^{+} \in \partial \bar{B}_{z}$, the $\vec{z}$-dependence of the curves $\left\{\left(\vec{y}_{\underline{l}}^{-}\left(\vec{z}, \vec{z}^{+}\right), \vec{z}\right) \mid \vec{z} \in B_{z}\right\}$ in the boundary of $V_{E}(\underline{l})$ is controlled by the $m$-uniform estimate (11.15), showing (12.5). Finally, the statements concerning $W_{E}\left(\underline{k}^{-}\right)$follow by time reversal $(10.5)$, since

$$
W_{E}\left(\underline{k}^{-}\right)=\mathcal{T} \mathcal{R}\left(V_{E}\left(k_{1}, k_{0}, k_{-1}, \ldots\right)\right) .
$$

Let

$$
\Lambda_{E}^{+}:=\bigcup_{\underline{k}^{+} \in \mathbf{X}^{+}} V_{E}\left(\underline{k}^{+}\right) \quad, \quad \Lambda_{E}^{-}:=\bigcup_{\underline{k}^{-} \in \mathbf{X}^{-}} W_{E}\left(\underline{k}^{-}\right)
$$

and

$$
\Lambda_{E}:=\Lambda_{E}^{+} \cap \Lambda_{E}^{-}
$$

By restriction, we associate to $\underline{k} \in \mathbf{X}$ half-infinite admissible sequences $\underline{k}^{ \pm} \in \mathbf{X}^{ \pm}$. Then we define a map $\mathcal{F}_{E}: \mathbf{X} \rightarrow \Lambda_{E}$ by

$$
\left.\mathcal{F}_{E}(\underline{k}):=V_{E}\left(\underline{k}^{+}\right) \cap W_{E} \underline{k}^{-}\right) \quad(k \in \mathbf{X}) .
$$

Note that in view of Proposition 11.5 the disks $V_{E}\left(\underline{k}^{+}\right)$and $W_{E}\left(\underline{k}^{-}\right)$in $\mathcal{H}_{E}^{k_{0}, k_{1}}$ intersect. The Lipschitz estimates of Lemma 12.1 imply that their intersection consists of precisely one point, which we identify with an element of $\Lambda_{E}$.

On $\mathbf{X}$ we introduced in (12.2) the metric $d$.

On the Poincaré surfaces $\mathcal{H}_{E}=\bigcup_{k \neq l} \mathcal{H}_{E}^{k, l}$ we use the metric

$$
\begin{aligned}
\text { dist } & : \mathcal{H}_{E} \times \mathcal{H}_{E} \rightarrow \mathbb{R} \\
\operatorname{dist}\left(x, x^{\prime}\right) & :=\left\{\begin{array}{cl}
\sqrt{\left(\vec{y}-\vec{y}^{\prime}\right)^{2}+\left(\vec{z}-\vec{z}^{\prime}\right)^{2}}, & x, x^{\prime} \in \mathcal{H}_{E}^{k, l} \\
1 & \text { otherwise }
\end{array}\right.
\end{aligned}
$$

based on the $(\vec{y}, \vec{z})$-coordinates (10.13) of $x$ and $x^{\prime}$ (Remark 10.3 showing that dist is well-defined).

Lemma 12.2. There exist $\alpha>0$ such that for $E>E_{\mathrm{th}}, \mathcal{F}_{E}$ is an $(\alpha \cdot \ln E)-H o ̈ l d e r$ continuous homeomorphism, that is,

$$
\operatorname{dist}\left(\mathcal{F}_{E}(\underline{k}), \mathcal{F}_{E}(\underline{l})\right) \leq C(E) \cdot d^{\alpha \ln E}(\underline{k}, \underline{l}), \quad(\underline{k}, \underline{l} \in \mathbf{X}),
$$

for some function $C>0$ of the energy, conjugating the shift with the restricted Poincaré map $\mathcal{P}_{E}^{\Lambda}:=\mathcal{P}_{E} \uparrow_{\Lambda_{E}}$ :

$$
\mathcal{F}_{E} \circ \sigma=\mathcal{P}_{E}^{\Lambda} \circ \mathcal{F}_{E}
$$


Proof. Since $C$ may depend on $E$, we can assume without loss of generality that the central blocks of $\underline{k}$ and $\underline{l}$ coincide, i.e. $\left(k_{-1}, k_{0}, k_{1}\right)=\left(l_{-1}, l_{0}, l_{1}\right)$. Let $I_{l}^{r} \subset \mathbb{Z}$ be the maximal interval containing 0 on which $\underline{k}$ and $\underline{l}$ coincide. Then by Lemma 11.7 and (11.18)

$$
\begin{aligned}
& \operatorname{dist}\left(\mathcal{F}_{E}(\underline{k}), \mathcal{F}_{E}(\underline{l})\right) \\
& \leq \operatorname{diam}\left(W_{E}\left(k_{l}, \ldots, k_{1}\right) \cap V_{E}\left(k_{0}, \ldots, k_{r}\right)\right) \\
& \leq 4\left(\operatorname{diam}_{y}\left(W_{E}\left(k_{l}, \ldots, k_{1}\right)\right)+\operatorname{diam}_{y}\left(V_{E}\left(k_{0}, \ldots, k_{r}\right)\right)\right) \\
& \leq 8 \frac{C}{E}\left[\left(\frac{1}{2} f_{\min } E\right)^{-|l|}+\left(\frac{1}{2} f_{\min } E\right)^{1-r}\right] \leq 4 f_{\min }^{2} C\left(\frac{1}{2} f_{\min } E\right)^{-\min (1-l, r+1)} \\
& =4 f_{\min }^{2} C \cdot 2^{-\left(\min (1-l, r+1) \frac{\ln \left(f_{\min } E / 2\right)}{\ln 2}\right)} \leq 4 f_{\min }^{2} C \cdot d^{\alpha \ln E}(\underline{k}, \underline{l})
\end{aligned}
$$

with $f_{\min }:=\min _{i \neq j \neq k}|f(i, j, k)|$, setting $\alpha$ slightly smaller than $1 / \ln (2)$.

For $n \geq 2$ we denote $T_{E} \circ \mathcal{F}_{E}: \mathbf{X} \rightarrow \mathbb{R}^{+}$, with the return time $T_{E}$ defined in (10.3) by $T_{E}$, too. Being defined by composition of a smooth map with a Hölder continuous map, $T_{E}$ is Hölder.

The continuous flow on $b_{E}$ is modelled as follows.

Definition 12.3. Given a roof function $r \in C^{0}\left(\mathbf{X}, \mathbb{R}^{+}\right)$, we set

$$
\mathbf{X}_{r}:=\mathbf{X} \times \mathbb{R} / \sim
$$

where $\sim$ is the equivalence relation defined by

$$
(\underline{k}, t+r(\underline{k})) \sim(\sigma(\underline{k}), t) \quad((\underline{k}, t) \in \mathbf{X} \times \mathbb{R}) .
$$

Then the $r$-suspension flow is given by

$$
\sigma_{r}^{t}: \mathbf{X}_{E} \rightarrow \mathbf{X}_{E}, \quad[(\underline{k}, s)] \mapsto[(\underline{k}, s+t)] \quad(t \in \mathbb{R}) .
$$

In the interesting case $r=T_{E}$ we abbreviate $\mathbf{X}_{E}:=\mathbf{X}_{T_{E}}$ and $\sigma_{E}^{t}:=\sigma_{T_{E}}^{t}$.

Definition 12.4. The set $\mathcal{C T}$ of collision times of a trajectory $c: I \rightarrow P$ is given by

$$
\mathcal{C T}(c):=\left\{t \in I \mid \vec{q} \circ c(t) \in\left\{\vec{s}_{1}, \ldots, \vec{s}_{n}\right\}\right\}
$$

The Morse index of a hyperbolic $T$-periodic trajectory $c:[0, T) \rightarrow P$ is given by

$$
\operatorname{Index}(c):=\sum_{t \in[0, T) \backslash \mathcal{C T}(c)} \operatorname{dim}\left(E_{u}(c(t)) \cap \operatorname{Vert}_{c(t)}\right)+|\mathcal{C} \mathcal{T}(c)|,
$$

where $E_{S / u}(c(t)) \subset T_{c(t)} P$ denotes the weak (un)stable subspace at $c(t)$ and the vertical subspace $\operatorname{Vert}_{x} \subset T_{x} P$ at $x \in T^{*} \hat{M} \subset P$ is the one annihilated by the linearized configuration space projection $T_{x} \vec{q}$. 
Remarks 12.5. 1) The weak (un)stable subspaces $E_{s / u}(c(t))$ are the direct sums of the (2-dim.) strong (un)stable subspaces and the neutrally stable flow direction (see $[\mathrm{KH}])$. Similar to $\operatorname{Vert}_{c(t)}$, they are thus 3-dim. Lagrangian subspaces of $T_{c(t)} P$.

2) Actually $\operatorname{Index}(c)$ is always finite. We already know from our study of the (near)collision process that $\mathcal{C T}(c)$ does not have accumulation points. On the other hand, the set of times $t$ where the (un)stable subspace $E_{s / u}(c(t))$ turns vertical is finite, too, since the kinetic energy term $\frac{1}{2} \vec{p}^{2}$ in the Hamiltonian function is a positive quadratic form, see Duistermaat [Du].

3) The additional term $|\mathcal{C T}(c)|$ in the definition of the Morse index is chosen in the only way that makes that definition invariant under small perturbations of the flow.

For $0<\theta \leq \pi$ we introduced in (9.1) the pericentric hypersurfaces

$$
\mathcal{H}_{l}(\theta)=\left\{x \in \mathcal{H}_{l}|| \vec{q}(x)-\vec{s}_{l} \mid<\frac{\left|Z_{l}\right|}{H(x) \cdot \sin (\theta / 4)}\right\} \quad(l=1, \ldots, n)
$$

(with $\mathcal{H}_{l}$ defined in (8.1)) near the $l$ th nucleus. So the angle parameter $\theta$ fixes the precise meaning of the term 'near-collision'.

Definition 12.6. The set $\mathcal{N C T}_{\theta}(c)$ of $\theta$-near-collision times of a trajectory $c: I \rightarrow P$ is given by

$$
\mathcal{N C T}_{\theta}(c):=\left\{t \in I \mid c(t) \in \cup_{n=1}^{n} \mathcal{H}_{l}(\theta)\right\} .
$$

We say that the trajectory $c \quad \theta$-visits the nuclei $\underline{k} \in \mathbf{X}_{l}^{r}$ in succession if $\mathcal{N C T}_{\theta}(c) \neq \varnothing$,

$$
r=\left|\mathcal{N C T}_{\theta}(c) \cap[0, \infty)\right| \quad, \quad l=1-\left|\mathcal{N C} T_{\theta}(c) \cap(-\infty, 0)\right|
$$

and

$$
c\left(t_{i}\right) \in \mathcal{H}_{k_{i}}(\theta) \quad\left(i \in I_{l}^{r}\right)
$$

for the enumeration $\mathcal{N C T}_{\theta}(c)=\left\{t_{i} \mid i \in I_{l}^{r}, t_{i}<t_{i+1}, t_{0}<0 \leq t_{1}\right\}$ of nearcollision times.

Remarks 12.7. 1) $\underline{\mathrm{k}}$ is really well-defined, since the hypersurfaces $\mathcal{H}_{l}(\theta) \subset \mathcal{H}_{l}$ do not intersect, and since the flow $\Phi^{t}$ is transversal to $\mathcal{H}_{l}$ so that $\mathcal{N C} T_{\theta}(c)$ is discrete.

2) As long as we analyze bounded motion, the natural angle will be $\theta=\alpha_{\min }$, so in that case we do not write it explicitly.

Lateron, in the analysis of the scattering process, $\theta$ will be a parameter, since scattering with small angles shows non-universal features.

Theorem 12.8. 1. For an NC configuration and $E>E_{\mathrm{th}}$ the map

$$
\mathcal{F}_{E}^{T}: \mathbf{X}_{E} \rightarrow b_{E}, \quad[(\underline{k}, s)] \mapsto \Phi^{s}\left(\mathcal{F}_{E}(\underline{k})\right)
$$

is a Hölder continuous homeomorphism conjugating the suspension flow with the flow on the set $b_{E}$ of energy $E$ bound states:

$$
\Phi^{t} \circ \mathcal{F}_{E}^{T}=\mathcal{F}_{E}^{T} \circ \sigma_{E}^{t} \quad(t \in \mathbb{R}) .
$$

Thus for 
- $n=1$ there are no bounded orbits,

- $n=2$ that set consists of one closed orbit: $b_{E} \cong S^{1}$

- $n \geq 3 \quad b_{E}$ is locally homeomorphic to the product of a Cantor set and an interval.

2. All bounded orbits are hyperbolic.

3. $b_{E}$ has measure zero w.r.t. Liouville measure $\lambda_{E}$ on $\Sigma_{E}$.

4. If a T-periodic trajectory c $:[0, T) \rightarrow \Sigma_{E}$ visits the nuclei $\left(k_{1}, \ldots, k_{m}\right) \in \mathbf{X}_{1}^{m}$ in succession, then its Morse index equals $\operatorname{Index}(c)=m$.

Proof. 1) By the virial identity (2.12) all trajectories that leave the interaction zone $\mathcal{I Z}$ go to spatial infinity and thus do not belong to $b_{E}$. Hence

$$
b_{E} \subset \mathcal{D}_{E} \quad \text { with } \quad \mathcal{D}_{E} \subset \Sigma_{E} \text { defined in (10.2). }
$$

Lemma 10.1 tells us that for $b_{E}$ the Poincaré return time $T_{E}$ is uniformly bounded by $\mathcal{O}(1 / \sqrt{E})$, so that a fortiori

$$
\Phi\left(T_{E}(x), x\right) \in \mathcal{H}_{E} \quad\left(x \in b_{E}\right) .
$$

Thus

$$
b_{E}=\Phi\left(\mathbb{R}, \Lambda_{E}\right),
$$

showing that $\mathcal{F}_{E}^{T}: \mathbf{X}_{E} \rightarrow b_{E}$ is a bijection. Using Lemma 12.2, we see that $\mathcal{F}_{E}^{T}$ is a Hölder continuous homeomorphism, since the flow $\Phi\left\lceil\Sigma_{E}\right.$ on the energy shell can locally be straightened out (see, e.g., [AM], Theorem 2.1.9), and since the Poincaré section $\mathcal{H}_{E}$ is transversal to the flow.

Now for $n=1$ the space $\mathbf{X}$ of admissible sequences is empty, and for $n=2$

$$
\mathbf{X}=\{(\ldots 12121 \ldots),(\ldots 21212 \ldots)\} \quad \text { so that } \quad \mathbf{X}_{E} \cong S^{1}
$$

Finally, for $n \geq 3$ the sequence space $\mathbf{X}$ is a Cantor set (a non-void compact totally disconnected set without isolated points, see, e.g. Katok and Hasselblatt [KH], A 1.)

2) The existence of the strictly invariant cone field $\mathcal{C}$ (defined in (11.1)) on $\Lambda_{E}$ implies the hyperbolicity of the flow. The expanding (as well as the contracting) subspace of $T_{x} \Sigma_{E}, x \in \Lambda_{E}$, is two-dimensional, since it equals

$$
\bigcap_{m \in \mathbb{N}} D \mathcal{P}_{E}^{m} \mathcal{C}\left(\mathcal{P}_{E}^{-m}(x)\right)
$$

3) The property of $b_{E} \subset \Sigma_{E}$ to have measure zero is defined without reference to a measure (since in every local chart of the smooth manifold $\Sigma_{E}$ the image of Liouville measure $\Lambda_{E}$ is continuous w.r.t. Lebesgue measure, see Hirsch [Hi], Chapt. 3.1). In fact, applying the Straightening Out Theorem ([AM], Theorem 2.1.9) it suffices to show that $\Lambda_{E}$ has measure zero. This follows for $E>E_{\text {th }}$ from estimate (12.8).

4) Setting $k_{0}:=k_{m}$, we denote by $s_{i}$ the intersection times with the Poincaré surfaces, that is, $c\left(s_{i}\right) \in \mathcal{H}_{E}^{k_{i-1}, k_{i}}(i=1, \ldots, m)$. 
We assume w.l.o.g. that the $T$-periodic trajectory $c:[0, T) \rightarrow \Sigma_{E}$ begins and ends in the Poincaré surface $\mathcal{H}_{E}^{k_{0}, k_{1}}$, so that we have $s_{i}<t_{i}<s_{i+1}$ and $s_{1}=0$. Additionally we set $s_{m+1}:=T$. Then we prove that $\operatorname{Index}(c)=m$ by showing that

$$
\sum_{t \in\left[s_{i}, s_{i+1}\right) \backslash \mathcal{C} \mathcal{T}(c)} \operatorname{dim}\left(E_{u}(c(t)) \cap \operatorname{Vert}_{c(t)}\right)+\left|\mathcal{C} \mathcal{T}(c) \cap\left[s_{i}, s_{i+1}\right)\right|=1 \quad(i=1, \ldots, m),
$$

which means that every near-collision adds one to the Morse index. This claim is equivalent to

$$
\sum_{t \in\left[s_{i}, s_{i+1}\right) \backslash\left\{t_{i}\right\}} \operatorname{dim}\left(E_{u}(c(t)) \cap \operatorname{Vert}_{c(t)}\right)=\left\{\begin{array}{l}
0, \vec{q}\left(t_{i}\right)=\vec{s}_{k_{i}} \\
1, \text { otherwise }
\end{array} \quad(i=1, \ldots, m),\right.
$$

where we used the coordinates $c(t) \equiv(\vec{p}(t), \vec{q}(t))$.

First we treat the case $Z_{k_{i}}<0$ of a repelling Coulomb singularity. There we know that the trajectory does not touch the singularity $\left(\vec{q}\left(t_{i}\right) \neq \vec{s}_{k_{i}}\right)$.

The simplest case is the one where along a neighbourhood of $\vec{q}\left(\left[s_{i}, s_{i+1}\right]\right)$ the potential $V$ coincides with the Keplerian potential $-Z_{l} /\left|\vec{q}-\vec{s}_{k_{i}}\right|$, and the angular momentum $\hat{L}_{k_{i}}=\left(\vec{q}(t)-\vec{s}_{k_{i}}\right) \times \vec{p}(t)$ of the trajectory relative to the nucleus vanishes.

Then $\vec{p}\left(t_{i}\right)=\overrightarrow{0}$, which gives a contribution of one in (12.11), since there the flow direction, given by the Hamiltonian vector field

$$
X_{H}\left(c\left(t_{i}\right)\right)=\left(-\nabla V\left(\vec{q}\left(t_{i}\right)\right), \vec{p}\left(t_{i}\right)\right) \in \operatorname{Vert}_{c\left(t_{i}\right)},
$$

is vertical.

However in that case there are no further contributions to the index. This may be seen as follows. The intersection of the (three-dimensional) weak unstable subspace at $c\left(s_{i}\right)$ with the tangent space to the local Poincaré section $\mathcal{H}_{E}^{k_{i}, k_{i+1}}$ is a two-dim. subspace which lies inside the local cone field $\mathcal{C}\left(c\left(s_{i}\right)\right)$ defined in (11.1). Thus we have $\delta \vec{q} \cdot \delta \vec{p} \geq 0$ for a variation vector

$$
(\delta \vec{p}, \delta \vec{q}) \in E_{u}\left(c\left(s_{i}\right)\right) \cap T_{c\left(s_{i}\right)} \mathcal{H}_{E}^{k_{i}, k_{i+1}} \subset T_{c\left(s_{i}\right)} P,
$$

and this property is preserved by the forward flow, since

$$
\frac{d}{d t}(\delta \vec{q}(t) \cdot \delta \vec{p}(t))=-\delta \vec{q}(t) \cdot D^{2} V(\vec{q}(t)) \delta \vec{q}(t)+\delta \vec{p}(t) \cdot \delta \vec{p}(t) \geq 0 .
$$

For general repelling potentials and general trajectories $c(12.11)$ equals one, too, by a continuity argument based on estimate (11.2).

Now we treat the case $Z_{k_{i}}>0$, again starting with a Keplerian potential $-Z_{l} /\left|\vec{q}-\vec{s}_{k_{i}}\right|$. and vanishing angular momentum $\hat{L}_{k_{i}}$. Here $\vec{q}\left(t_{i}\right)=\vec{s}_{k_{i}}$, so that we have to show that $E_{u}(c(t))$ does not turn vertical for $t \in\left[s_{i}, s_{i+1}\right) \backslash\left\{t_{i}\right\}$. However, the configuration space trajectories $\vec{q}\left(\left[s_{i}, t_{i}\right]\right)$ and $\vec{q}\left(\left[t_{i}, s_{i+1}\right]\right)$ are straight lines, and the infinitesimal two-parameter family of diverging Kepler hyperbolae corresponding to initial conditions (12.12) do not have a conjugate point. A more formal way of 
seeing this is to use the Jacobi metric $g_{E}(\vec{q})=(1-V(\vec{q}) / E) g(\vec{q})$ discussed in (A.1) of the Appendix. Then the energy $E$ solution curves correspond to geodesics $c$ in that metric, and the linearization of the flow $\Phi_{t}$ corresponds to the Jacobi equation

$$
\nabla^{2} \vec{Y}(t)+R_{\dot{c}(t)} \vec{Y}(t)=\overrightarrow{0}
$$

see, e.g., [Kl]. For a variational vector field $\vec{Y}$ orthonormal to the geodesic velocity vector $\dot{c}(t)$ the self-adjoint curvature operator $R_{\dot{c}(t)}$ has an $\vec{Y}$-expectation value equal to the sectional curvature of the plane spanned by these two vectors $([\mathrm{Kl}]$, Proposition 1.11.3). The sectional curvature of $g_{E}$ has been calculated in (A.2) of the Appendix.

For the geodesic under consideration the relevant plane contains the direction $\vec{q}-\vec{s}_{k_{i}}$ of the singularity and is thus seen to be negative definite (setting $q_{3}=0$ in (A.2)).

However, negative sectional curvature, together with initial conditions (12.12), lead to absence of conjugate points. This proves (12.11) for the collision orbit.

In the case of non-vanishing angular momentum $\hat{L}_{k_{i}}$. the sectional curvature (A.2)) is still negative in the plane perpendicular to $\hat{L}_{k_{i}}$. However, near the singularity (A.2)) becomes positive for plane containing the vector $\hat{L}_{k_{i}}$, leading to a conjugate point. So (12.11) holds for that case, too.

Like in the case $Z_{k_{i}}<0$, a continuity argument based on estimate (11.2) shows assertion 4. for general potentials $V$ and large $E$.

\section{Fractal dimension}

In this section we estimate the fractional dimension of the set $b_{E}$ of energy $E$ bound states, for $E$ large. This quantity, being of interest in its own right, governs the measure of those scattering orbits which have a large time delay.

\section{Dimensions: definitions and elementary properties}

Besides the well-known dimension $\operatorname{dim}_{\mathcal{H}}$ introduced by Hausdorff and Besicovitch, there exist several other definitions of the fractional dimension. Of those we will only consider upper box-counting dimension $\operatorname{dim}_{\mathcal{B}}$, since most dimensions take values between $\operatorname{dim}_{\mathcal{H}}$ and $\operatorname{dim}_{\mathcal{B}}$, see Falconer $[\mathrm{Fa}]$.

Definition 13.1. Let $(X, d)$ be a separable metric space and $U \subset X, U \neq \emptyset$. The diameter $\operatorname{diam}(U)$ of $U$ is given by

$$
\operatorname{diam}(U):=\sup \{d(x, y) \mid x, y \in U\} \quad \text { and } \operatorname{diam}(\varnothing):=0 .
$$

For $E \subset X, s \geq 0$ and $\delta>0$ let

$$
\mathcal{H}_{\delta}^{s}(E):=\inf \left\{\sum_{i=1}^{\infty}\left(\operatorname{diam}\left(U_{i}\right)\right)^{s} \mid E \subset \cup_{i \in \mathbb{N}} U_{i}, \operatorname{diam}\left(U_{i}\right) \leq \delta\right\}
$$


(setting $0^{0}:=1$ except for the case $\operatorname{diam}(\emptyset)^{0}:=0$ ). The Hausdorff s-dimensional outer measure of $E, \mathcal{H}^{s}(E)$, is then defined by

$$
\mathcal{H}^{s}(E):=\sup _{\delta>0} \mathcal{H}_{\delta}^{s}(E)=\lim _{\delta \searrow 0} \mathcal{H}_{\delta}^{s}(E) .
$$

The Hausdorff dimension of $E$ is given by

$$
\operatorname{dim}_{\mathcal{H}}(E):=\sup \left\{s \in \mathbb{R} \mid \mathcal{H}^{s}(E)=\infty\right\}=\sup \left\{s \in \mathbb{R} \mid \mathcal{H}^{s}(E)>0\right\} .
$$

The important property of $\mathcal{H}^{s}$ is that it defines a measure on, say, the Borel sets. Therefore, Hausdorff dimension is not only monotone, that is

$$
E_{1} \subset E_{2} \Rightarrow \operatorname{dim}_{\mathcal{H}}\left(E_{1}\right) \leq \operatorname{dim}_{\mathcal{H}}\left(E_{2}\right),
$$

but also countably stable:

$$
\operatorname{dim}_{\mathcal{H}}\left(\cup_{i \in \mathbb{N}} E_{i}\right)=\sup _{i \in \mathbb{N}} \operatorname{dim}_{\mathcal{H}}\left(E_{i}\right) .
$$

Definition 13.2. Let $(X, d)$ be a manifold $X$ with metric $d$ and $E \subset X$ be a nonempty bounded subset. The (upper) box-counting dimension (or Minkowski dimension) $\operatorname{dim}_{\mathcal{B}}(E)$ is given by

$$
\operatorname{dim}_{\mathcal{B}}(E):=\limsup _{\varepsilon \downarrow 0} \frac{\ln \left(N_{\varepsilon}(E)\right)}{-\ln (\varepsilon)},
$$

where $N_{\varepsilon}(E)$ is the minimal number of balls of radius $\varepsilon$ needed to cover $E$.

$\operatorname{dim}_{\mathcal{B}}$ is monotone (see (13.3)), and

$$
\operatorname{dim}_{\mathcal{B}}\left(E_{1} \cup E_{2}\right)=\max \left(\operatorname{dim}_{\mathcal{B}}\left(E_{1}\right), \operatorname{dim}_{\mathcal{B}}\left(E_{2}\right)\right),
$$

but it is not countably stable, since $\operatorname{dim}_{\mathcal{B}}$ is invariant under closure. Furthermore, for all (bounded) $E$,

$$
\operatorname{dim}_{\mathcal{H}}(E) \leq \operatorname{dim}_{\mathcal{B}}(E)
$$

Example 13.3. The triadic Cantor set $E \subset \mathbb{R}$ has

$$
\operatorname{dim}_{\mathcal{H}}(E)=\operatorname{dim}_{\mathcal{B}}(E)=\frac{\ln 2}{\ln 3}=0.6309 \cdots .
$$

On the other hand, the set $\mathbb{Q} \subset \mathbb{R}$ of rational numbers has dimensions

$$
\operatorname{dim}_{\mathcal{H}}(\mathbb{Q})=0 \quad, \quad \operatorname{dim}_{\mathcal{B}}(\mathbb{Q} \cap[0,1])=1 .
$$


We have

$$
\operatorname{dim}_{\mathcal{H}}(f(E)) \leq \operatorname{dim}_{\mathcal{H}}(E) \text { and } \operatorname{dim}_{\mathcal{B}}(f(E)) \leq \operatorname{dim}_{\mathcal{B}}(E)
$$

if the map $f$ is Lipschitz. In general both dimensions are not additive w.r.t. cartesian products, but the inequalities

$$
\operatorname{dim}_{\mathcal{H}}\left(E_{1}\right)+\operatorname{dim}_{\mathcal{H}}\left(E_{2}\right) \leq \operatorname{dim}_{\mathcal{H}}\left(E_{1} \times E_{2}\right) \leq \operatorname{dim}_{\mathcal{H}}\left(E_{1}\right)+\operatorname{dim}_{\mathcal{B}}\left(E_{2}\right)
$$

and

$$
\operatorname{dim}_{\mathcal{B}}\left(E_{1} \times E_{2}\right) \leq \operatorname{dim}_{\mathcal{B}}\left(E_{1}\right)+\operatorname{dim}_{\mathcal{B}}\left(E_{2}\right)
$$

hold true, see Mattila [Ma] and Tricot [Tr].

\section{Lower estimate for the Hausdorff dimension}

By (13.6) we need a lower estimate for the Hausdorff dimension and an upper estimate for the box counting dimension.

Whereas any choice of coverings by $\varepsilon$-balls leads to an upper estimate for $\operatorname{dim}_{\mathcal{B}}$, a lower estimate for $\operatorname{dim}_{\mathcal{H}}$ is provided by the mass distribution principle:

Proposition (see [Fa]). Let $\mu$ be a probability measure on $(X, d)$ and $\mu(E)>0$. Suppose that for some $s \geq 0, C>0$ and $\delta>0$

$$
\mu(U) \leq C \cdot \operatorname{diam}(U)^{s} \quad(\operatorname{diam}(U) \leq \delta),
$$

Then $\operatorname{dim}_{\mathcal{H}}(E) \geq s$.

Proof. If $E \subset \cup_{i \in \mathbb{N}} U_{i}$, then

$$
\mu(E) \leq \mu\left(\cup_{i \in \mathbb{N}} U_{i}\right) \leq \sum_{i=1}^{\infty} \mu\left(U_{i}\right) \leq C \sum_{i=1}^{\infty} \operatorname{diam}\left(U_{i}\right)^{s}
$$

so that $0<\mu(E) \leq C \mathcal{H}_{\delta}^{s}(E)$. Then the statement follows from the second expression for $\operatorname{dim}_{\mathcal{H}}$ in (13.2).

\section{Thermodynamic formalism for dimension estimates}

We base ourselves on the size estimate Proposition 11.5 for $V_{E}(\underline{k})$ in terms of the finite geometric data encoded in $f$ (see (11.3)).

Hence we consider the parameter-dependent $(n(n-1) \times n(n-1))$-matrix $M(s)$ with double-indices in $\{(i, j) \in \mathcal{S} \times \mathcal{S} \mid i \neq j\}$ and entries

$$
M(s)_{i, j ; k, l}:=\left\{\begin{array}{cl}
|f(i, k, l)|^{-s} & , i \neq j, k \neq l \text { and } j=k \\
0 & , \text { otherwise }
\end{array} .\right.
$$


For $s \in \mathbb{R}$ this is a matrix with non-negative entries, and for $n \geq 3$ centres all entries of $(M(s))^{m}$ are strictly positive iff $m \geq 3$. Thus by the Perron-Frobenius (PF) Theorem $M(s)$ has a unique eigenvalue $\lambda_{\max }(s)$ of largest modulus, which is positive and of multiplicity one, and the corresponding eigenvector $v(s)$ can be chosen to have strictly positive entries.

In the case $n=2$ the two eigenvalues of $M(s)$ are given by $\pm \lambda_{\max }(s)=$ $\pm|f(1,2,1)|^{-s}$.

Lemma 13.4. For all $E>E_{\mathrm{th}}$ there is a unique solution $d(E):=s$ of the equation

$$
\lambda_{\max }(s)=E^{s},
$$

and $d(E)=0$ for $n=2$, whereas for $n \geq 3$

$$
d(E)=\frac{\ln (n-1)}{\ln (E)} \cdot\left(1+\mathcal{O}\left(\frac{1}{\ln (E)}\right)\right) .
$$

Finally

$$
d^{\prime}(E)=-\frac{\ln (n-1)}{E(\ln (E))^{2}}\left(1+\mathcal{O}\left(\frac{1}{\ln (E)}\right)\right)
$$

Proof. We conjugate $M(s) / E^{s}$ with the $(n(n-1) \times n(n-1))-$ matrix $D(s)$ given by

$$
D(s)_{i, j ; k, l}:=\left\{\begin{array}{cl}
\left(d^{i, j}\right)^{s / 2} & , i=k \neq j=l \\
0 & , \text { otherwise }
\end{array} .\right.
$$

Then $\tilde{M}(s, E):=D(s) M(s) D(s)^{-1} / E^{s}$ has the non-negative entries

$$
\tilde{M}(s, E)_{i, j ; k, l}=\left\{\begin{array}{cl}
(\tilde{f}(i, k, l) E)^{-s} & , i \neq j, k \neq l \text { and } j=k \\
0 & , \text { otherwise }
\end{array}\right.
$$

with

$$
\tilde{f}(i, k, l):=\sqrt{d^{k, l} / d^{i, k}}|f(i, k, l)|>0 \quad(i \neq k \neq l) .
$$

The right Perron-Frobenius eigenvector of $\tilde{M}(s, E)$ equals

$$
\tilde{v}(s):=D(s) v(s) \quad \text { with eigenvalue } \quad \tilde{\lambda}_{\max }(s, E):=\lambda_{\max }(s) / E^{s},
$$

and (13.10) corresponds to the implicit equation

$$
\tilde{\lambda}_{\max }(d(E), E)=1 .
$$

Although $\tilde{f}(l, k, i)=\tilde{f}(i, k, l)$ by def. (11.3) of $f$, for $n>2$ the matrix $\tilde{M}(s, E)$ is non-symmetric and even non-normal, leading to slightly more complicated estimates. 
We denote the left PF eigenvector by $\tilde{w}(s)$, again assuming positivity of its entries. Then for $E$ large $\tilde{\lambda}_{\max }(s, E)$ is strictly decreasing in $s$, since

$$
D_{1} \tilde{\lambda}_{\max }(s, E)=\frac{\left\langle\tilde{w}(s), D_{1} \tilde{M}(s, E) \tilde{v}(s)\right\rangle}{\langle\tilde{w}(s), \tilde{v}(s)\rangle}<0,
$$

$D_{i}$ denoting the derivative w.r.t. the $i$ th argument.

Inequality (13.15) follows from the fact that all components of $\tilde{v}(s)$ and $\tilde{w}(s)$ are positive, and for $E$ large all entries of $D_{1} \tilde{M}(s, E)$ are non-positive, and some are negative. So the 1.h.s. of the equation $\tilde{\lambda}_{\max }(s, E)=1$ is strictly decreasing in $s$. Furthermore,

$$
\tilde{\lambda}_{\max }(0, E)=n-1 \geq 1 \quad \text { and } \quad \lim _{s \rightarrow \infty} \tilde{\lambda}_{\max }(s, E)=0 .
$$

This implies that (13.10) has a unique solution $d(E)=0$ for $n=2$ and $d(E)>0$ for $n \geq 3$.

More precisely we observe that the $n-1$ non-vanishing entries in each row $(i, j)$ of $\tilde{M}(s, E)$ are of the form $(\tilde{f} E)^{-s}$. Setting

$$
\tilde{f}_{\min }:=\min _{i \neq k \neq l} \tilde{f}(i, k, l) \quad \text { and } \quad \tilde{f}_{\max }:=\max _{i \neq k \neq l} \tilde{f}(i, k, l),
$$

we note that by a consideration of the largest and the smallest components of the eigenvalue equation $\tilde{\lambda}_{\max }(s, E) \tilde{v}(s)=\tilde{M}(s, E) \tilde{v}(s)$

$$
\frac{\ln (n-1)}{\ln \left(\tilde{f}_{\max } E\right)} \leq d(E) \leq \frac{\ln (n-1)}{\ln \left(\tilde{f}_{\min } E\right)} .
$$

This shows the estimate (13.11). The implicit equation (13.14) for $d(E)$ shows that

$$
d^{\prime}(E)=\frac{-D_{2} \tilde{\lambda}_{\max }(d(E), E)}{D_{1} \tilde{\lambda}_{\max }(d(E), E)}=\frac{d(E)}{E \cdot D_{1} \tilde{\lambda}_{\max }(d(E), E)} .
$$

So we have to estimate

$$
D_{1} \tilde{\lambda}_{\max }(d(E), E)=\left.\frac{\left\langle\tilde{w}(s), D_{1} \tilde{M}(s, E) \tilde{v}(s)\right\rangle}{\langle\tilde{w}(s), \tilde{v}(s)\rangle}\right|_{s=d(E)}
$$

more precisely in order to show (13.12). The matrix

$$
\mathcal{M}:=\lim _{E \rightarrow \infty} \tilde{M}(d(E), E)
$$

has the form

$$
\mathcal{M}_{i, j ; k, l}=\left\{\begin{array}{cl}
1 /(n-1) & , i \neq j, k \neq l \text { and } j=k \\
0 & , \text { otherwise }
\end{array},\right.
$$

since by (13.16) the quotients

$$
\begin{aligned}
\tilde{M}(d(E), E)_{i, k ; k, l} / \tilde{M}(d(E), E)_{i^{\prime}, k^{\prime} ; k^{\prime}, l^{\prime}} & =\left(\tilde{f}\left(i^{\prime}, k^{\prime}, l^{\prime}\right) / \tilde{f}(i, k, l)\right)^{d(E)} \\
& =1+\mathcal{O}(1 / \ln (E))
\end{aligned}
$$


of the non-zero coefficients converge to one, and since the PF eigenvalue of $\tilde{M}(d(E), E)$ equals one. We now use the algebraic relation

$$
((n-1) \mathcal{M})^{2}+(n-1) \mathcal{M}=\mathcal{F} \quad \text { with } \quad \mathcal{F}_{i, j ; k, l}:=1 \quad(i \neq j, k \neq l),
$$

which is approximately met for finite energies in the sense that by (13.19)

$$
\begin{aligned}
R(E) & :=((n-1) \tilde{M}(d(E), E))^{2}+(n-1) \tilde{M}(d(E), E) \\
& =\mathcal{F}+\mathcal{O}(1 / \ln (E)) .
\end{aligned}
$$

By (13.14) the PF eigenvectors fulfil the equations

$$
R(E) \tilde{v}(d(E))=n(n-1) \tilde{v}(d(E)) \quad, \quad R(E)^{t} \tilde{w}(d(E))=n(n-1) \tilde{w}(d(E))
$$

so that in view of estimate (13.20) its components are nearly equal:

$$
\tilde{v}(d(E))_{i, j}=\frac{\sum_{k \neq l} \tilde{v}(d(E))_{k, l}}{n(n-1)} \cdot(1+\mathcal{O}(1 / \ln (E))) \quad(i \neq j)
$$

and similarly for $\tilde{w}$.

Finally, for $i \neq j, k \neq l$ and $j=k$

$$
D_{1} \tilde{M}(d(E), E)_{i, j ; k, l}=-\ln (|\tilde{f}(i, k, l) E|) /(n-1) \cdot(1+\mathcal{O}(1 / \ln (E))) .
$$

Inserting that estimate and (13.21) into (13.18) we obtain

$$
D_{1} \tilde{\lambda}_{\max }(d(E), E)=-\ln (E)+\mathcal{O}(1) .
$$

Putting that estimate and (13.16) into (13.17) gives (13.12).

Dimensions of the set $b_{E}$ of bound states

We now estimate $\operatorname{dim}_{\mathcal{B}}\left(b_{E}\right)$ and $\operatorname{dim}_{\mathcal{H}}\left(b_{E}\right)$ by the solution $d(E)$ of the matrix eigenvalue problem (13.10).

Theorem 13.5. For $E>E_{\text {th }}$ and $n \geq 3$ the Hausdorff dimension $\operatorname{dim}_{\mathcal{H}}$ and the upper box-counting dimension $\operatorname{dim}_{\mathcal{B}}$ of the energy $E$ bound states $b_{E}$ meet the estimates

$$
\begin{aligned}
& 1+2 d(E) \cdot\left(1-\mathcal{O}\left((E \ln E)^{-1}\right)\right) \leq \operatorname{dim}_{\mathcal{H}}\left(b_{E}\right) \leq \\
& \quad \leq \operatorname{dim}_{\mathcal{B}}\left(b_{E}\right) \leq 1+2 d(E) \cdot\left(1+\mathcal{O}\left((E \ln E)^{-1}\right)\right)
\end{aligned}
$$

with the solution $d(E)$ of (13.10). In particular they meet the rough estimate

$$
\operatorname{dim}_{\mathcal{H}}\left(b_{E}\right)=1+\frac{2 \ln (n-1)}{\ln (E)}+\mathcal{O}\left((\ln E)^{-2}\right)=\operatorname{dim}_{\mathcal{B}}\left(b_{E}\right)
$$

For $n=2$ centres $\operatorname{dim}_{\mathcal{H}}\left(b_{E}\right)=\operatorname{dim}_{\mathcal{B}}\left(b_{E}\right)=1$. 
Proof. The second inequality in (13.22) is the abstract inequality (13.6). Estimate (13.23) follows from (13.22) by inserting (13.11).

So it remains to prove the lower bound for $\operatorname{dim}_{\mathcal{H}}$ and then the upper bound for $\operatorname{dim}_{\mathcal{B}}$. For $n=2$ centres $b_{E}$ consists of one closed orbit, whose dimensions equal one. So we assume from now on $n \geq 3$.

1) Using the constant $\delta E$ from Proposition 11.5 and setting $E_{L}:=E+\delta E$ for $E>E_{\text {th }}$, estimate (13.12) shows that

$$
d(E)=d_{L} \cdot(1-\mathcal{O}(1 /(E \ln E))) \quad \text { for } \quad d_{L}:=d\left(E_{L}\right),
$$

so that the first inequality in (13.22) follows from an estimate

$$
\operatorname{dim}_{\mathcal{H}}\left(b_{E}\right) \geq 1+2 d_{L} \quad\left(E>E_{\mathrm{th}}\right) .
$$

We show that this follows from

$$
\operatorname{dim}_{\mathcal{H}}\left(\Lambda_{E}\right) \geq 2 d_{L} \quad\left(E>E_{\text {th }}\right) .
$$

First we find $\varepsilon>0$ such that the flow $\Phi: \mathbb{R} \times P \rightarrow P$, restricted to $(-\varepsilon, \varepsilon) \times \mathcal{H}_{E}$, is a diffeomorphism onto its image. Thus

$$
U_{\varepsilon}:=\Phi\left((-\varepsilon, \varepsilon) \times \Lambda_{E}\right) \subset b_{E} .
$$

satisfies

$$
\begin{aligned}
\operatorname{dim}_{\mathcal{H}}\left(U_{\varepsilon}\right) & =\operatorname{dim}_{\mathcal{H}}\left((-\varepsilon, \varepsilon) \times \Lambda_{E}\right) \\
& \leq \operatorname{dim}_{\mathcal{H}}((-\varepsilon, \varepsilon))+\operatorname{dim}_{\mathcal{H}}\left(\Lambda_{E}\right)=1+\operatorname{dim}_{\mathcal{H}}\left(\Lambda_{E}\right)
\end{aligned}
$$

using (13.7) and (13.8). By finiteness of the Poincaré return time $T_{E}$ (Lemma 10.1), $b_{E}$ can be covered by finitely many time translates

$$
b_{E}=\bigcup_{j=0}^{j_{\max }} \Phi\left(\varepsilon j, U_{\varepsilon}\right) \quad \text { with } \quad j_{\max }:=\left[\varepsilon^{-1} \sup _{x} T_{E}(x)\right]
$$

so that

$$
\operatorname{dim}_{\mathcal{H}}\left(b_{E}\right)=\max _{j} \operatorname{dim}_{\mathcal{H}}\left(\Phi\left(\varepsilon j, U_{\varepsilon}\right)\right)=\operatorname{dim}_{\mathcal{H}}\left(U_{\varepsilon}\right)
$$

follows from stability of $\operatorname{dim}_{\mathcal{H}}$ and (13.7). So we are reduced to show (13.25), by employing the mass distribution principle. This will be based on a $\mathcal{P}_{E}^{\Lambda}$-invariant probability measure $\mu_{E}$ on $\Lambda_{E}$, which is the image

$$
\mu_{E}:=\mathcal{F}_{E} \mu_{\mathbf{X}, E}
$$

w.r.t. (12.7) of a measure $\mu_{\mathbf{X}, E}$ on $\mathbf{X} . \mu_{\mathbf{X}, E}$ is defined through its values

$$
\mu_{\mathbf{X}, E}(Z(\underline{k})):=\tilde{w}\left(d_{L}\right)_{k_{l}, k_{l+1}} \cdot \prod_{i=l+1}^{r-1}\left(\tilde{f}\left(k_{i-1}, k_{i}, k_{i+1}\right) E_{L}\right)^{-d_{L}} \quad\left(\underline{k} \in \mathbf{X}_{l}^{r}\right)
$$


on the cylinder sets

$$
Z(\underline{k}):=\left\{\underline{k}^{\prime} \in \mathbf{X} \mid k_{i}^{\prime}=k_{i} \forall i \in\{l, \ldots, r\}\right\} \quad\left(l<r \in \mathbb{Z}, \underline{k} \in \mathbf{X}_{l}^{r}\right),
$$

with the left Perron-Frobenius eigenvector $\tilde{w}\left(d_{L}\right)$ of $\tilde{M}\left(d_{L}, E_{L}\right)$.

Using the $l^{1}$-normalization

$$
\sum_{i \neq k} \tilde{w}(s)_{i, k}=1
$$

of $\tilde{w}(s)$ and the relations (13.13) and (13.14) we see that the definitions (13.28) are compatible and define a $\sigma$-invariant Borel probability measure $\mu_{\mathbf{X}, E}$. By the conjugacy (12.9) between the shift $\sigma$ and the restricted Poincaré map the image measure $\mu_{E}$ is then indeed $\mathcal{P}_{E}^{\Lambda}$-invariant.

We now claim that the mass distribution principle applies. Namely we have for some $C \equiv C(E)>0$ and $\delta \equiv \delta(E)>0$

$$
\mu_{E}(U) \leq C \cdot \operatorname{diam}(U)^{2 d_{L}}
$$

for all measurable $U \subset \Lambda_{E}$ of small diameter diam $(U)<\delta$. Instead of general such $U$ we first consider balls $B\left(\delta^{\prime}\right) \subset \mathcal{H}_{E}$ of small radius $\delta^{\prime}>0$, which are centered at a point $x \in \Lambda_{E}$. The symbol sequence $\underline{k}:=\mathcal{F}_{E}^{-1}(x) \in \mathbf{X}$ of this point projects to the half-infinite sequences $\underline{k}^{ \pm} \in \mathbf{X}^{ \pm}$(see (12.7)). There are unique integers $l<0<r$ with

$$
\begin{gathered}
C_{6}^{-1} E_{L}^{-1} \prod_{i=l+1}^{0}\left(\tilde{f}\left(k_{i-1}, k_{i}, k_{i+1}\right) E_{L}\right)^{-1}>\delta^{\prime} \\
\text { but } \quad C_{6}^{-1} E_{L}^{-1} \prod_{i=l}^{0}\left(\tilde{f}\left(k_{i-1}, k_{i}, k_{i+1}\right) E_{L}\right)^{-1} \leq \delta^{\prime} .
\end{gathered}
$$

respectively

$$
\begin{aligned}
& C_{6}^{-1} E_{L}^{-1} \prod_{i=1}^{r-1}\left(\tilde{f}\left(k_{i-1}, k_{i}, k_{i+1}\right) E_{L}\right)^{-1}>\delta^{\prime} \\
& \text { but } C_{6}^{-1} E_{L}^{-1} \prod_{i=1}^{r}\left(\tilde{f}\left(k_{i-1}, k_{i}, k_{i+1}\right) E_{L}\right)^{-1} \leq \delta^{\prime},
\end{aligned}
$$

By the lower estimates (13.31) and (13.33) and (11.19) of Proposition 11.5

$$
V_{E}\left(k_{0}^{\prime}, \ldots, k_{r}^{\prime}\right) \cap B\left(\delta^{\prime}\right) \neq \emptyset \quad \text { only if } \quad\left(k_{0}^{\prime}, \ldots, k_{r}^{\prime}\right)=\left(k_{0}, \ldots, k_{r}\right) .
$$

and similarly for $W_{E}\left(k_{l}^{\prime}, \ldots, k_{1}^{\prime}\right)$. However, this implies that

$$
B\left(\delta^{\prime}\right) \cap \Lambda_{E} \subset \mathcal{F}_{E}\left(Z\left(k_{l}, \ldots, k_{r}\right)\right) .
$$


Thus by def. (13.28) of $\mu_{\mathbf{X}, E}$ and the upper estimates (13.32) and (13.34)

$$
\begin{aligned}
\mu_{E}\left(B\left(\delta^{\prime}\right)\right) & \leq \mu_{\mathbf{X}, E}\left(Z\left(k_{l}, \ldots, k_{r}\right)\right) \\
& =\tilde{w}\left(d_{L}\right)_{k_{l}, k_{l+1}} \cdot \prod_{i=l+1}^{r-1}\left(\tilde{f}\left(k_{i-1}, k_{i}, k_{i+1}\right) E_{L}\right)^{-d_{L}} \\
& \leq c_{\alpha} \cdot\left(2 \delta^{\prime}\right)^{2 d_{L}}=c_{\alpha} \cdot \operatorname{diam}\left(B\left(\delta^{\prime}\right)\right)^{2 d_{L}}
\end{aligned}
$$

the constant

$$
c_{\alpha}:=\left(\max _{a \neq b} \tilde{w}\left(d_{L}\right)_{a, b}\right) \cdot\left(C_{6} E_{L}^{2} \tilde{f}_{\max }\right)^{2 d_{L}}
$$

being independent of $\delta^{\prime}$ and $\underline{k}$ (remembering that $\left.\tilde{f}_{\max }=\max _{i \neq k \neq l} \tilde{f}(i, k, l)\right)$. That is, the balls $B\left(\delta^{\prime}\right)$ satisfy the mass distribution estimate (13.30).

General sets $U \subset \mathcal{H}_{E}$ of diameter $\frac{1}{2} \delta^{\prime}$ and $U \cap \Lambda_{E} \neq \emptyset$ are subsets of such balls of radius $\delta^{\prime}$ centered at a point in $U \cap \Lambda_{E}$. This proves the mass distribution principle (13.30) in general.

We see from (13.30) that for any cover $\Lambda_{E} \subset \cup_{i} U_{i}$ with $\operatorname{diam}\left(U_{i}\right)<\delta$,

$$
1 \leq \mu_{E}\left(\Lambda_{E}\right) \leq \sum_{i} \mu_{E}\left(U_{i}\right) \leq C \sum_{i} \operatorname{diam}\left(U_{i}\right)^{2 d_{L}}
$$

which implies $\mathcal{H}^{2 d_{L}}\left(\Lambda_{E}\right) \geq 1 / C$ and thus $\operatorname{dim}_{\mathcal{H}}\left(\Lambda_{E}\right) \geq 2 d_{L}$.

2) Similar to the first case, to obtain the upper bound in (13.22) for $\operatorname{dim}_{\mathcal{B}}\left(b_{E}\right)$, it suffices to show that for $\delta E>0$ from Proposition 11.5

$$
\operatorname{dim}_{\mathcal{B}}\left(b_{E}\right) \leq 1+2 d_{U}
$$

with

$$
d_{U}:=d(E-2 \delta E) \quad \text { and } \quad E_{U}:=E-\delta E .
$$

Also (13.35) follows from the estimate

$$
\operatorname{dim}_{\mathcal{B}}\left(\Lambda_{E}\right) \leq 2 d_{U},
$$

for then $\operatorname{dim}_{\mathcal{B}}\left(U_{\varepsilon}\right) \leq 1+2 d_{U}$ (for the set $U_{\varepsilon}=\Phi\left((-\varepsilon, \varepsilon) \times \Lambda_{E}\right)$ ) is a consequence of (13.7) and (13.9). Relation (13.5) may then be used to determine the box dimension of the covering (13.27).

We now claim that for $\varepsilon>0$ small we can cover $\Lambda_{E}$ in the form

$$
\Lambda_{E} \subset \bigcup_{\underline{k} \in I_{\varepsilon}} \mathcal{F}_{E}(Z(\underline{k}))
$$


with index set

$$
\begin{aligned}
I_{\varepsilon}:=\left\{\underline{k} \in \bigcup_{l=-1}^{-\infty} \bigcup_{r=1}^{\infty} \mathbf{X}_{l}^{r} \mid\right. \\
\frac{\varepsilon}{16} \geq C_{6} E^{-1} \prod_{i=l+1}^{0}\left(\left|f\left(k_{i-1}, k_{i}, k_{i+1}\right)\right| \cdot E_{U}\right)^{-1} \geq \frac{\varepsilon}{16 f_{\max } E_{U}}, \\
\left.\frac{\varepsilon}{16} \geq C_{6} E^{-1} \prod_{i=1}^{r-1}\left(\left|f\left(k_{i-1}, k_{i}, k_{i+1}\right)\right| \cdot E_{U}\right)^{-1} \geq \frac{\varepsilon}{16 f_{\max } E_{U}}\right\} .
\end{aligned}
$$

- To prove (13.38), we choose an arbitrary bi-infinite sequence $\underline{l} \in \mathbf{X}$, and show that there is a $\underline{k} \in I_{\varepsilon}$ with $\underline{l} \in Z(\underline{k})$.

Since we have chosen a small $\varepsilon$, the right inequalities in the definition of $I_{\varepsilon}$ are met for $l=-1$ and $r=1$. We find $\underline{k}$ by setting $k_{i}:=l_{i}$ and choosing $r$ such that the right inequality would be violated for $r+1$. Then the left inequality holds. Similarly we choose the minimal possible $l<0$.

- Next we claim that for $\underline{k} \in I_{\varepsilon}$ the subset $\mathcal{F}_{E}(Z(\underline{k}))$ of the Poincaré section is contained in a ball of radius $\varepsilon$. This holds true since

$$
\mathcal{F}_{E}(Z(\underline{k}))=W_{E}\left(k_{l}, \ldots, k_{1}\right) \cap V_{E}\left(k_{0}, \ldots, k_{r}\right),
$$

see (12.7), so that using Lemma 11.7 and (11.18)

$$
\begin{aligned}
& \operatorname{diam}\left(\mathcal{F}_{E}(Z(\underline{k}))\right) \\
& \leq 4\left(\operatorname{diam}_{y}\left(W_{E}\left(k_{l}, \ldots, k_{1}\right)\right)+\operatorname{diam}_{y}\left(V_{E}\left(k_{0}, \ldots, k_{r}\right)\right)\right) \\
& \leq 8\left(C_{6} E^{-1} \prod_{i=l+1}^{0}\left(\left|f\left(k_{i-1}, k_{i}, k_{i+1}\right)\right| \cdot E_{U}\right)^{-1}\right. \\
& \left.\quad+C_{6} E^{-1} \prod_{i=1}^{r-1}\left(\left|f\left(k_{i-1}, k_{i}, k_{i+1}\right)\right| \cdot E_{U}\right)^{-1}\right) \leq \varepsilon .
\end{aligned}
$$

- Finally, denoting by $b:=1 /\left(16 f_{\max } E_{U}\right)$ the constant appearing in the definition of $I_{\varepsilon}$, the number $N_{\varepsilon}\left(\Lambda_{E}\right)$ of $\varepsilon$-balls needed to cover $\Lambda_{E}$ is bounded by

$$
\begin{aligned}
& N_{\varepsilon}\left(\Lambda_{E}\right) \leq\left|I_{\varepsilon}\right| \\
& \leq \mid\left\{\underline{k} \in \bigcup_{l=-1}^{-\infty} \bigcup_{r=1}^{\infty} \mathbf{X}_{l}^{r} \mid C_{6} E^{-1} \prod_{i=l+1}^{0}\left(\left|f\left(k_{i-1}, k_{i}, k_{i+1}\right)\right| \cdot E_{U}\right)^{-1} \geq b \varepsilon\right. \\
& \left.C_{6} E^{-1} \prod_{i=1}^{r-1}\left(\left|f\left(k_{i-1}, k_{i}, k_{i+1}\right)\right| \cdot E_{U}\right)^{-1} \geq b \varepsilon\right\}||
\end{aligned}
$$




$$
\begin{aligned}
\leq \mid\{\underline{k} & \left.\in \bigcup_{l=-1}^{-\infty} \bigcup_{r=1}^{\infty} \mathbf{X}_{l}^{r} \mid C_{6}^{2} E^{-2} \prod_{i=l+1}^{r-1}\left(\left|f\left(k_{i-1}, k_{i}, k_{i+1}\right)\right| \cdot E_{U}\right)^{-1} \geq(b \varepsilon)^{2}\right\} \mid \\
& \leq\left(\varepsilon b E / C_{6}\right)^{-2 d_{U}} \sum_{l=-1}^{-\infty} \sum_{r=1}^{\infty} \sum_{k \in \mathbf{X}_{l}^{r}} \prod_{i=l+1}^{r-1}\left(\tilde{f}\left(k_{i-1}, k_{i}, k_{i+1}\right) E_{U}\right)^{-d_{U}} \\
& =\left(\varepsilon b E / C_{6}\right)^{-2 d_{U}} \sum_{m=1}^{\infty} m \cdot\left\langle\overrightarrow{\mathbb{1}}, \tilde{M}\left(d_{U}, E_{U}\right)^{m} \overrightarrow{\mathbb{1}}\right\rangle
\end{aligned}
$$

with the matrix $\tilde{M}(s, E)$ from (13.13), and $\overrightarrow{1} \in \mathbb{R}^{n(n-1)}$ denoting the vector whose components equal 1. Now for the arguments (13.36) the PerronFrobenius eigenvalue $\tilde{\lambda}_{\max }(s, E)$ of $\tilde{M}(s, E)$ is smaller than one:

$$
\begin{aligned}
\tilde{\lambda}_{\text {max }}\left(d_{U}, E_{U}\right) & =\frac{\lambda_{\max }\left(d_{U}\right)}{E_{U}^{d_{U}}}=\frac{\lambda_{\max }(d(E-2 \delta E))}{E_{U}^{d_{U}}} \\
& <\frac{\lambda_{\max }(d(E-2 \delta E))}{(E-2 \delta E)^{d_{U}}}=1 .
\end{aligned}
$$

In view of the near-constancy (13.21) of the left PF eigenvector $\tilde{v} \equiv \tilde{v}\left(d\left(E_{U}\right)\right)$ of $\tilde{M} \equiv \tilde{M}\left(d_{U}, E_{U}\right)$ we use the inequality

$$
\left\langle\overrightarrow{\mathbb{1}}, \tilde{M}^{m} \overrightarrow{\mathbb{1}}\right\rangle \leq 2 n(n-1) \frac{\left\langle\tilde{v}, \tilde{M}^{m} \tilde{v}\right\rangle}{\langle\tilde{v}, \tilde{v}\rangle} \quad(m \in \mathbb{N}),
$$

to exchange the vector $\overrightarrow{11}$ by $\tilde{v}$ :

$$
\begin{aligned}
N_{\varepsilon}\left(\Lambda_{E}\right) & \leq\left(\varepsilon b E / C_{6}\right)^{-2 d_{U}} 2 n(n-1) \sum_{m=1}^{\infty} m \cdot \frac{\left\langle\tilde{v}, \tilde{M}\left(d_{U}, E_{U}\right)^{m} \tilde{v}\right\rangle}{\langle\tilde{v}, \tilde{v}\rangle} \\
& =\left(\varepsilon b E / C_{6}\right)^{-2 d_{U}} 2 n(n-1) \sum_{m=1}^{\infty} m \cdot \tilde{\lambda}_{\max }\left(d_{U}, E_{U}\right)^{m} \\
& \leq C^{\prime} \varepsilon^{-2 d_{U}}
\end{aligned}
$$

with finite $C^{\prime}$, using (13.39). Inserting this into the Definition 13.2 of the box-counting dimension shows (13.37).

\section{Topological entropy}

We shall now determine the topological entropy of the flow $\Phi_{E}^{t}=\Phi^{t} \uparrow_{\Sigma_{E}}$ on the energy shell $\Sigma_{E}$.

The estimate of $h_{\text {top }}\left(\Phi_{E}^{1}\right)$ is based on Proposition 14.3, stating that the topological entropy of the flow is determined by its restriction to the set $b_{E}$ of bound states. It is then relatively easy to compute that topological entropy using symbolic dynamics. 
First we formally introduce the notion of topological entropy of $T: X \rightarrow X$, following the definition of Bowen. That definition is in a way more general than others in allowing for non-compact metric spaces $(X, d)$ (see Walters [Wa]).

Definition 14.1. Let $(X, d)$ be a metric space and $T: X \rightarrow X$ a uniformly continuous map. Then for $m \in \mathbb{N}, \varepsilon>0$, a subset $F \subset X$ is said to $(m, \varepsilon)$-span a compact $K \subset X$ if

$$
\forall x \in K \exists y \in F: d_{m}(x, y) \leq \varepsilon,
$$

with the metric $d_{m}(x, y):=\max _{0 \leq i \leq m-1} d\left(T^{i} x, T^{i} y\right)$.

Let $r_{m}(\varepsilon, T, K)$ be the smallest cardinality of an $(m, \varepsilon)$-spanning set $F$ of $K$,

$$
h_{r}(\varepsilon, T, K):=\limsup _{m \rightarrow \infty} \frac{1}{m} \ln \left(r_{m}(\varepsilon, T, K)\right),
$$

and $h_{\text {top }}(T, K):=\lim _{\varepsilon \rightarrow 0} h_{r}(\varepsilon, T, K)$.

Then the topological entropy of $T$ is

$$
h_{\text {top }}(T):=\sup \left\{h_{\text {top }}(T, K) \mid K \subset X \text { compact }\right\} \text {. }
$$

Note that $h_{r}(\varepsilon, T, K)$ is monotonically increasing in $\varepsilon$, so that its limit $h_{\text {top }}(T, K)$ exists (it can be $\infty$ ).

If $d^{\prime}$ is a second metric on $X$ uniformly equivalent to $d$, that is, if the maps

$$
\mathrm{Id}:(X, d) \rightarrow\left(X, d^{\prime}\right) \quad \text { and } \quad \mathrm{Id}:\left(X, d^{\prime}\right) \rightarrow(X, d)
$$

are both uniformly continuous, then the topological entropies $h_{\text {top }}(T, d)$ and $h_{\text {top }}\left(T, d^{\prime}\right)$ coincide, see [Wa]. Therefore, for compact metrisable spaces $X$ the topological entropy only depends on the topology generated by the metric.

We are to estimate the topological entropy for the time-one flow $T:=\boldsymbol{\Phi}_{E}^{1}$ on the energy shell $X:=\Sigma_{E}$, and for any metric on $\Sigma_{E}$ uniformly equivalent outside the compact region projecting to the interaction zone to the metric induced by the Euclidean metric of $T^{*}\left(\mathbb{R}^{3}-\mathcal{I Z}\right) . T$ is uniformly continuous since the motion is asymptotically free.

In our case we have

$$
h_{\text {top }}\left(\boldsymbol{\Phi}_{E}^{-1}\right)=h_{\text {top }}\left(\boldsymbol{\Phi}_{E}^{+1}\right) \text {, }
$$

although in general $h_{\text {top }}\left(\boldsymbol{\Phi}_{E}^{-1}, K\right) \neq h_{\text {top }}\left(\boldsymbol{\Phi}_{E}^{+1}, K\right)$, since we have a symmetry w.r.t. time reversal.

Proposition 14.3 below will show the importance of the set $b_{E}$ of energy $E$ bound states.

Definition 14.2. The non-wandering set $\Omega(\Phi)$ of a continuous flow $\Phi^{t}: X \rightarrow X$ is given by

$$
\Omega(\Phi):=\left\{x \in X \mid \forall \text { neighb. } U \ni x, \forall T>0: U \cap\left(\bigcup_{t \geq T} \Phi^{t}(U)\right) \neq \emptyset\right\} .
$$


For non-compact spaces $X$ the topological entropy is in general larger than the topological entropy of the restriction to the non-wandering set. Nevertheless, in our case we have

Proposition 14.3. For $E>E_{\mathrm{th}}$, the non-wandering set equals

$$
\Omega\left(\Phi_{E}\right)=b_{E},
$$

and the topological entropy of the flow on the energy shell is determined by the bound states, that is, $h_{\text {top }}\left(\Phi_{E}^{1}\right)=0$ for $n=1$ centre and

$$
h_{\text {top }}\left(\Phi_{E}^{1}\right)=h_{\text {top }}\left(\Phi_{E}^{1} \uparrow_{b_{E}}\right)
$$

for $n \geq 2$.

Proof. The proof parallels the one of Lemma 7.4 of [KK]. In particular $h_{\text {top }}\left(\Phi_{E}^{1}\right)=0$ for $n=1$, since then by Theorem $12.8 b_{E}=\emptyset$.

Theorem 14.4. 1) For $E>E_{\text {th }}$ and $n=1$ or $n=2$ centres the topological entropy equals $h_{\text {top }}\left(\Phi_{E}^{1}\right)=0$.

2) For $n \geq 3$ centres

$$
h_{\text {top }}\left(\Phi_{E}^{1}\right)=h_{\text {top }}^{\infty} \cdot \sqrt{2 E} \cdot\left(1+\frac{\ln (E)}{E} C_{h_{\text {top }}}+\mathcal{O}(1 / E)\right) .
$$

Here $h_{\text {top }}^{\infty}$ is the unique solution (with eigenvector $\vec{v}$ ) of the largest eigenvalue problem $\lambda(s)=1$ for the one parameter family of $n \times n$ Perron-Frobenius matrices $\mathcal{M}(s)$,

$$
\begin{gathered}
\mathcal{M}(s)_{i, j}:=\left\{\begin{array}{c}
\exp \left(-s d^{i, j}\right) i \neq j \\
0 \quad \begin{array}{r}
i=j
\end{array}
\end{array}\right. \\
C_{h_{\text {top }}}:=\frac{\langle\vec{v}, \vec{Z}\rangle\langle\overrightarrow{\mathbb{1}}, \vec{v}\rangle}{2\langle\vec{v}, D \vec{v}\rangle} \text { with } \vec{Z}:=\left(Z_{1}, \ldots, Z_{n}\right), \overrightarrow{\mathbb{1}}:=(1, \ldots, 1)
\end{gathered}
$$

and the matrix $D$ of distances $d^{i, j}$.

3) Let $N_{E}(T)$ be the number of closed $\Phi_{E}^{t}$-orbits of period smaller than $T$. Then there exists a (E-independent) constant $C>1$ so that for $T$ large

$$
C^{-1} \frac{\exp \left(h_{\text {top }} T\right)}{h_{\text {top }} T} \leq N_{E}(T) \leq C \frac{\exp \left(h_{\text {top }} T\right)}{h_{\text {top }} T}, \quad\left(E>E_{\text {th }}\right)
$$

with $h_{\text {top }} \equiv h_{\text {top }}\left(\Phi_{E}^{1}\right)$.

Remark 14.5. For configurations which form an equilateral triangle or a tetrahedron, the trivial estimate

$$
h_{\text {top }}^{\infty} \in\left[\frac{\ln (n-1)}{d_{\max }}, \frac{\ln (n-1)}{d_{\min }}\right]
$$

for the PF eigenvalue problem of the matrices $\mathcal{M}(s)$ is sharp. 
Proof. 1) For $n=1$, by Proposition 14.3 the topological entropy is zero for $E$ large. By Proposition 14.3, for $n \geq 2$ we need only estimate $h_{\text {top }}\left(\Phi_{E}^{1} \uparrow_{b_{E}}\right)$.

For $n=2$, we know from Theorem 12.8 that $b_{E}$ consists of only one closed orbit. Therefore, by Theorem 7.14 of [Wa], the topological entropy $h_{\text {top }}\left(\Phi_{E}^{1}\right)=$ $h_{\text {top }}\left(\Phi_{E}^{1} \uparrow_{b_{E}}\right)=0$, too.

2) The interesting case left is $n \geq 3$. Since $h_{\text {top }}$ is a conjugacy invariant (Theorem 7.2 of [Wa]), we have by Theorem 12.8

$$
h_{\text {top }}\left(\Phi_{E}^{1} \uparrow_{b_{E}}\right)=h_{\text {top }}\left(\sigma_{E}^{1}\right)
$$

for the time-one $T_{E}$-suspension flow $\sigma_{E}^{1}$ on $\mathbf{X}_{E}$ (see (12.10)).

Generally, given a roof function $r: \mathbf{X} \rightarrow \mathbb{R}^{+}$(see Definition 12.3), any $\sigma$ invariant probability measure $\mu$ on $\mathbf{X}$ induces a probability measure $\mu_{r}$ on $\mathbf{X}_{r}$ which is invariant under the $r$-suspension flow $\sigma_{r}^{t}$, namely the one obtained by normalization of the measure $\mu \times d x$ on $\mathbf{X} \times \mathbb{R}$, restricted to the fundamental domain $\{(\underline{k}, t) \mid 0 \leq t<r(\underline{k})\} \cong \mathbf{X}_{r}$. Conversely, any $\sigma_{r}^{t}$-invariant probability measure can be obtained in that way.

Furthermore, the topological entropy is the supremum of the $K S$-entropies over all ergodic measures, and the same is generally true for the topological pressure

$$
\begin{aligned}
P(T, f) & :=\sup \left\{h_{\mu}(T)+\int_{X} f d \mu \mid \mu T-\text { invariant prob. measure }\right\} \\
& =\sup \left\{h_{\mu}(T)+\int_{X} f d \mu \mid \mu \text { ergodic w.r.t. } T\right\}
\end{aligned}
$$

of a continuous map $T: X \rightarrow X$ of a compact metrisable space $X$ and $f \in C(X, \mathbb{R})$, see Corollary 9.10.1 of [Wa].

Finally, any $\sigma_{r}^{t}$-ergodic measure $\mu_{r}$ on $\mathbf{X}_{r}$ comes from a $\sigma$-ergodic measure $\mu$ on $\mathbf{X}$.

So we can apply Abramov's formula

$$
h_{\mu_{r}}\left(\sigma_{r}^{1}\right)=\left(\int_{\mathbf{X}} r d \mu\right)^{-1} h_{\mu}(\sigma),
$$

(which is valid for any ergodic measure $\mu$ on $\mathbf{X}$, see Theorem 2.1 of Chap. 3 in Sinai $[\operatorname{Sin}])$, to obtain the formula

$$
h_{\text {top }}\left(\sigma_{r}^{1}\right)=\sup \left\{h_{\mu}(\sigma) / \int_{\mathbf{X}} r d \mu \mid \mu \text { ergodic }\right\} .
$$

Eq. (14.7) implies the scaling behaviour

$$
h_{\text {top }}\left(\sigma_{\lambda r}^{1}\right)=\lambda^{-1} h_{\text {top }}\left(\sigma_{r}^{1}\right) \quad\left(\lambda \in \mathbb{R}^{+}\right)
$$

and the inequality

$$
h_{\mathrm{top}}\left(\sigma_{r_{1}}^{1}\right) \geq h_{\mathrm{top}}\left(\sigma_{r_{2}}^{1}\right) \quad\left(r_{1} \leq r_{2}\right) .
$$


Using Lemma 10.6 for estimating the Poincaré return time $T_{E}$, we obtain for $C$ large

$$
(1-C / E) \cdot r(E) \leq \sqrt{2 E} \cdot T_{E} \leq(1+C / E) \cdot r(E) \quad\left(E>E_{\mathrm{th}}\right),
$$

with

$$
r(E) \in C\left(\mathbf{X}, \mathbb{R}^{+}\right) \quad, \quad r(E)(\underline{k}):=\frac{1}{2}\left(d^{k_{-1}, k_{0}}+d^{k_{0}, k_{1}}-Z_{k_{0}} \frac{\ln E}{E}\right) \quad(\underline{k} \in \mathbf{X}) .
$$

So by applying (14.8) and (14.9), we get

$$
h_{\text {top }}\left(\sigma_{E}^{1}\right)=h_{\text {top }}\left(\sigma_{r(E)}^{1}\right) \cdot \sqrt{2 E} \cdot(1+\mathcal{O}(1 / E)) .
$$

The claim (14.2) follows from (14.10) and (14.5), if we can prove

$$
h_{\text {top }}\left(\sigma_{r(E)}^{1}\right)=h_{\text {top }}^{\infty} \cdot\left(1+\frac{\ln (E)}{E} C_{h_{\text {top }}}+\mathcal{O}(1 / E)\right) \text {. }
$$

This is done by reformulating it as a question about the topological pressure $P(\sigma,-s \cdot r(E))$ for the shift $\sigma$. We know that for $s=0$

$$
P(\sigma, 0)=h_{\text {top }}(\sigma)=\ln (n-1)>0,
$$

since $\ln (n-1)$ is the largest eigenvalue of the transition matrix of the shift, and since $n \geq 3$. On the other hand, for $E \geq E_{\text {th }}$ the values $r(E)(\underline{k})$ are larger than $d_{\min } / 2$, so that the roof function $r \equiv r(E) \in C\left(\mathbf{X}, \mathbb{R}^{+}\right)$has mean $\int_{X} r d \mu \geq d_{\min } / 2$ w.r.t. to any probability measure $\mu$. Thus $P(\sigma,-s \cdot r) \leq 0$ for $s \geq 2 \ln (n-1) / d_{\min }$, and there exists an $s>0$ with

$$
P(\sigma,-s \cdot r)=0
$$

By convexity of the map $f \mapsto P(\sigma, f)$ (Theorem 9.7(v) of [Wa]) this $s$ is unique.

Moreover the roof function $r$ is locally constant, so that there exists a unique equilibrium state $\mu$ for (14.12) (see [Ru]), i.e.

$$
h_{\mu}(\sigma)=s \int_{X} r d \mu \text { and } h_{\nu}(\sigma)<s \int_{X} r d \nu \text { for } \quad \nu \neq \mu .
$$

Thus $h_{\text {top }}\left(\sigma_{r}^{1}\right)=s$, using (14.7) (and ergodicity of $\mu$, which follows from uniqueness, see Theorem 9.13 of [Wa]). Since $\sigma: \mathbf{X} \rightarrow \mathbf{X}$ is an expansive homeomorphism, by Theorem 9.6 of [Wa] we can use the formula

$$
P(\sigma, f)=\lim _{m \rightarrow \infty} \frac{1}{m} \ln \left(p_{m}(\sigma, f, \alpha)\right),
$$

with the generating partition

$$
\alpha=\left\{A_{1}, \ldots, A_{n}\right\} \quad \text { with atoms } \quad A_{l}:=\left\{\underline{k} \in \mathbf{X} \mid k_{0}=l\right\}
$$


and

$$
p_{m}(\sigma, f, \alpha):=\inf \left\{\sum_{B \in \beta} \sup _{\underline{k} \in B} \exp \left(S_{m} f(\underline{k})\right) \mid \beta \text { finite subcover of } \bigvee_{i=0}^{m-1} \sigma^{-i} \alpha\right\} .
$$

Here $S_{m} f:=\sum_{i=0}^{m-1} f \circ \sigma^{i}$. Now for $\delta:=\ln (E) / E$

$$
\begin{gathered}
\exp \left(-s \cdot S_{m} r(E)(\underline{k})\right)= \\
\exp \left(-\frac{s}{2}\left(d^{k_{-1}, k_{0}}-d^{k_{m-1}, k_{m}}+\left(Z_{k_{m}}-Z_{k_{0}}\right) \frac{1}{2} \delta\right)\right) \cdot \prod_{i=0}^{m-1} \mathcal{M}(s, \delta)_{k_{i}, k_{i+1}},
\end{gathered}
$$

with the $n \times n$-matrix $\mathcal{M}(s, \delta)$ given by

$$
\mathcal{M}(s, \delta)_{i, j}:=\left\{\begin{aligned}
\exp \left(-s\left(d^{i, j}-\frac{\delta}{4}\left(Z_{i}+Z_{j}\right)\right)\right), & i \neq j \\
0 & , i=j
\end{aligned}\right.
$$

So the function is constant on the atoms of the partition $\beta:=\bigvee_{i=-1}^{m} T^{-i} \alpha$. The first factor in (14.13) is bounded from below and from above, uniformly in $m$. The PF property of the symmetric matrix $\mathcal{M}(s)$ then implies that $P(\sigma,-s \cdot r(E))$ is equal to the logarithm of the largest eigenvalue $\lambda(s, \delta)$ of $\mathcal{M}(s, \delta)$.

We now do perturbation theory around $\mathcal{M}(s, 0)=\mathcal{M}(s)$, with $\mathcal{M}(s)$ defined in (14.3), and obtain from the condition $\lambda(s(\delta), \delta)=1$

$$
s^{\prime}(\delta)=-\frac{D_{2} \lambda(s(\delta), \delta)}{D_{1} \lambda(s(\delta), \delta)} .
$$

Since the PF eigenvalue is isolated, the derivatives exist, and since $\mathcal{M}(s, \delta)$ is symmetric, they equal at $\delta=0$

$$
D_{1} \lambda(s(0), 0)=\left\langle\vec{v}, D_{1} \mathcal{M}(s(0), 0) \vec{v}\right\rangle=-\langle\vec{v}, D \vec{v}\rangle
$$

and

$$
D_{2} \lambda(s(0), 0)=\left\langle\vec{v}, D_{2} \mathcal{M}(s(0), 0) \vec{v}\right\rangle=\frac{1}{2} h_{\text {top }}^{\infty}\langle\vec{v}, \vec{Z}\rangle\langle\overrightarrow{1}, \vec{v}\rangle
$$

So

$$
s(\delta)=s(0)+s^{\prime}(0) \delta+\mathcal{O}\left(\delta^{2}\right)=h_{\mathrm{top}}^{\infty} \cdot\left(1+C_{h_{\mathrm{top}}} \delta+\mathcal{O}\left(\delta^{2}\right)\right),
$$

proving (14.11).

3) Finally, for fixed energy $E>E_{\text {th }}$ the estimate (14.4) for the number of periodic orbits follows from Theorem 2 of the article [PP] by Parry and Pollicott (or alternatively by arguments based on the Renewal Theorem): If the Axiom A flow $\sigma_{E}^{t}$ is topologically weak-mixing, then

$$
N_{E}(T) \sim \frac{\exp \left(h_{\mathrm{top}} T\right)}{h_{\mathrm{top}} T} .
$$


Otherwise all periods are known to be integral multiples of some $T_{0}>$, and the formula

$$
N_{E}(T) \sim \frac{h_{\text {top }} T_{0}}{1-\exp \left(-h_{\text {top }} T_{0}\right)} \frac{\exp \left(h_{\text {top }}\left[\frac{T}{T_{0}}\right] T_{0}\right)}{h_{\text {top }} T}
$$

follows directly from Theorem 2 of $[\mathrm{PP}]$. Here $T_{0}$ is the largest positive eigenperiod, which is bounded by

$$
T_{0} \leq \inf _{\underline{k} \in \mathbf{X}} T_{E}(\underline{k})=\mathcal{O}(1 / \sqrt{E}) .
$$

As we just proved that $h_{\text {top }}=\mathcal{O}(\sqrt{E}), h_{\text {top }} \cdot T_{0}$ has an $E$-independent upper bound, and we may thus use an $E$-independent $C>1$ in (14.4) to bound the r.h.s. of (14.14) from above and from below.

Remarks 14.6. 1) Observe that, up to a small error term, $h_{\text {top }}\left(\Phi_{E}^{1}\right)$ is independent of the charges $Z_{l}$ of the centres. Effectively an attracting potential speeds up the particle a bit, which then leads to the $\vec{Z}$-dependent correction term in (14.2).

2) It is natural to ask whether one may improve (14.4) to show

$$
N_{E}(T) \sim \frac{\exp \left(h_{\text {top }} T\right)}{h_{\text {top }} T} .
$$

However, the return time estimate of Lemma 10.6 for $T_{E}(x)$ is, up to the relative order $\mathcal{O}(1 / E)$, independent of the point $x \in V_{E}\left(k_{-1}, k_{0}, k_{1}\right)$.

So we cannot exclude by that estimate that all return times are equal to a constant $T_{R}$ for a symmetric configuration (e.g., for an equilateral triangle or tetrahedron with equal charges $Z_{l}$ ). But by formula (14.14) in that case $N_{E}(T)$ is not asymptotic to any smooth function, so that (14.15) does not hold. In this sense our statement is optimal, given the, already quite precise, return time estimate.

Any eventual improvement of Lemma 10.6 must be dependent of the additional smooth component $W$ of the potential $V$, and thus be complicated.

Although the iterated Poincare map is certainly mixing for the measure of maximal entropy, by the above argument we cannot decide whether or not the flow is mixing.

\section{Characterization of the scattering orbits}

Up to now we were mainly concerned with the bound states $b_{E} \subset \Sigma_{E}$. However, the topological entropy analyzed in the last section is an example for a quantity which, though a priori depending on the dynamics on the whole energy shell $\Sigma_{E}$, is determined by that subset of Liouville measure zero.

When we now consider the scattering states $s_{E} \subset \Sigma_{E}$, again their description will be based on symbolic dynamics of the bound states.

Our concrete question will be to enumerate the scattering orbits with given energy $E>0$ and asymptotic directions $\hat{\theta}^{ \pm} \in S^{2}$, that is, the subset

$$
\left(H, \hat{p}^{-}, \hat{p}^{+}\right)^{-1}\left(E, \hat{\theta}^{-}, \hat{\theta}^{+}\right)
$$


of phase space (noticing that the asymptotic $\hat{p}^{ \pm}$defined in (6.14) are constant on orbits).

Before we come to the case of several centres, we first consider the simple

Example 15.1. Keplerian motion. Without loss of generality $V(\vec{q})=-\frac{Z}{|\vec{q}|}$. There are no undeflected orbits, i.e. only asymptotic directions

$$
\left(\hat{\theta}^{-}, \hat{\theta}^{+}\right) \in\left(S^{2} \times S^{2}\right) \backslash \Delta, \quad \text { with diagonal } \quad \Delta:=\left\{(\hat{\theta}, \hat{\theta}) \mid \hat{\theta} \in S^{2}\right\}
$$

occur. The pericentric time $T$ is a smooth function on the positive energy part

$$
P_{+}:=\{x \in P \mid H(x)>0\}
$$

of phase space, see (5.7) and the definition of the manifold $P$. In fact in that case the map

$$
\left.\left(H, \hat{p}^{-}, \hat{p}^{+}, T\right)\right|_{P_{+}}: P_{+} \rightarrow \mathbb{R}^{+} \times\left(\left(S^{2} \times S^{2}\right) \backslash \Delta\right) \times \mathbb{R}
$$

is a diffeomorphism. In particular for $E>0$ and asymptotic directions $\hat{\theta}^{-} \neq \hat{\theta}^{+}$ there is exactly one Kepler hyperbola.

If we allow for an additional smooth potential $W$, we need to exclude a whole neighbourhood of the diagonal $\Delta \subset S^{2} \times S^{2}$, since even for large energies forward scattering may be dominated by $W$ and not by the Coulomb potential.

Already in the case of bounded orbits we introduced the NC condition of Definition 2.4 which excluded collinear configurations of nuclei. Similarly we need to exclude certain asymptotic directions if we want to obtain statements which are independent of the precise form of the potential $V$.

Thus for $\vartheta \in(0, \pi]$ and

$$
\Theta_{\min }: S^{2} \rightarrow[0, \pi] \quad \Theta_{\min }(\hat{\theta}):=\min _{1 \leq i \neq k \leq n} \varangle\left(\hat{\theta}, \hat{s}^{i, k}\right)
$$

(letting $\Theta_{\min }(\hat{\theta}):=\pi$ for $n=1$ nucleus) we restrict our interest to the asymptotic directions in

$$
\mathcal{A D}(\vartheta):=\left\{\left(\hat{\theta}^{-}, \hat{\theta}^{+}\right) \in S^{2} \times S^{2} \mid \min \left(\varangle\left(\hat{\theta}^{-}, \hat{\theta}^{+}\right), \Theta_{\min }\left(\hat{\theta}^{-}\right), \Theta_{\min }\left(\hat{\theta}^{+}\right)\right)>\vartheta\right\},
$$

and the ( $\Phi^{t}$-invariant) subset

$$
s_{E}(\vartheta):=\left\{x \in s_{E} \mid\left(\hat{p}^{-}(x), \hat{p}^{+}(x)\right) \in \mathcal{A D}(\vartheta)\right\}
$$

of scattering states, thus excluding near-forward scattering and scattering from or to any direction near an axis through two nuclei.

For $n \geq 2$ centres we have bound states which influence the scattering trajectories in their vicinity. As the high energy bound states can be described by symbolic dynamics, we introduce the set

$$
\mathcal{W}:=\bigcup_{k \in \mathbb{N}} \mathbf{X}_{1}^{k}
$$

of (non-empty) admissible words to enumerate the scattering states, see (12.1). 
Every orbit within $s_{E}(\vartheta)$ will be uniquely characterized by its asymptotic directions and by the sequence of its near-collisions with the nuclei $(\theta$-visits in the sense of Definition 12.6). Conversely every admissible word will be shown to occur for some orbit.

Namely we set the angle parameter controlling the asymptotic directions $\mathcal{A D}$ equal to

$$
\vartheta \equiv \vartheta(E):=\min \left(C_{7} / \sqrt{E}, \alpha_{\min } / 2\right)
$$

with $C_{7}$ to be fixed in Theorem 15.3.

Similarly, the angle parameter controlling the near-collisions is fixed by

$$
\theta \equiv \theta(E):=\min \left(C_{9} / \sqrt{E}, \alpha_{\min } / 2\right) \quad \text { with } \quad C_{9}:=4 c_{1} .
$$

In Lemma 8.4 the parameter $c_{1}$ controlled what the regime of hard scattering. It will be fixed in Theorem 15.3, too.

For $x \in s_{E}$ let $w(x):=\emptyset$ if $\mathcal{N C T}_{\theta}(x)=\emptyset$. Otherwise the orbit through $x$ enters the interaction zone, i.e. intersects the hypersurface $\partial \mathcal{D}_{E}^{-}$at a unique point $x^{\prime}$, and we set

$$
w(x):=\underline{k}\left(x^{\prime}\right) \in \mathcal{W},
$$

where the trajectory $t \mapsto \Phi^{t}\left(x^{\prime}\right) \theta$-visits the centres in succession $\underline{k}$ (we start at $x^{\prime}$ instead of $x$ since we want the $\theta$-visits to occur for positive times so that $\underline{k} \in \mathbf{X}_{1}^{r}$ for some $r$ ).

Lemma 15.2. For $C_{8}>0$ large and $E>E_{\text {th }}$ every orbit in $s_{E}\left(C_{8} / E\right)$ intersects the hypersurfaces $\partial \mathcal{D}_{E}^{ \pm}$exactly once, so that the entrance, resp. exit times $T_{E}^{ \pm}$on $s_{E}\left(C_{8} / E\right)$ are uniquely defined by $\Phi\left(T_{E}^{ \pm}(x), x\right) \in \partial \mathcal{D}_{E}^{ \pm}$.

The restrictions of $T_{E}^{+}$and $T_{E}^{-}$to $s_{E}\left(C_{8} / E\right)$ are smooth functions.

Proof. If a scattering orbit in $s_{E}$ does not meet the interaction zone, then by the virial estimate $(2.15)$ there is a unique point $x \equiv(\vec{p}, \vec{q})$ on this orbit with $\langle\vec{p}, \vec{q}\rangle=0$. By (2.13) the speed is bounded below by $|\vec{p}| \geq \sqrt{E}$. Thus the direction $\hat{p}=\vec{p} /|\vec{p}|$ differs from the asymptotic directions $\hat{p}^{ \pm}(x)$ (defined in (6.14)) only by

$$
\varangle\left(\hat{p}, \hat{p}^{ \pm}(x)\right)=\mathcal{O}(1 / E),
$$

using (6.6). Choosing a large enough constant $C_{8}$, we see that

$$
\varangle\left(\hat{p}, \hat{p}^{-}(x)\right)+\varangle\left(\hat{p}, \hat{p}^{+}(x)\right)<C_{8} / E \quad \text { if } E>E_{\mathrm{th}} .
$$

Thus the orbits in $s_{E}\left(C_{8} / E\right)$ intersect $\partial \mathcal{D}_{E}^{ \pm}$.

For these scattering states we know from (2.15) that there is a unique entrance resp. exit time. As long as the orbits intersect the $C^{\infty}$-hypersurfaces $\partial \mathcal{D}_{E}^{ \pm}$ transversally, smoothness of $T_{E}^{ \pm}$follows from smoothness of the flow $\Phi^{t}$. But this transversality can be enforced by further enlarging $C_{8}$. 
Theorem 15.3. We assume that $V$ satisfies the decay estimates (6.16). Then for $C_{7}>0$ large in (15.1), and $E>E_{\text {th }}$ the map

$$
\begin{aligned}
\operatorname{Diff}_{E}: s_{E}(\vartheta(E)) & \rightarrow \mathcal{A D}(\vartheta(E)) \times \mathcal{W} \times \mathbb{R} \\
x & \mapsto\left(\hat{p}^{-}(x), \hat{p}^{+}(x), w(x), T_{E}^{-}(x)\right)
\end{aligned}
$$

is a diffeomorphism.

Remarks 15.4. 1) In particular the orbits in $s_{E}(\vartheta(E))$ are uniquely characterized by their asymptotic directions and the sequence of near-collisions, and any sequence is realized by an orbit.

2) In this section and Sect. 16 we exclude from our consideration small cones of aperture $\mathcal{O}(1 / \sqrt{E})$ around the axes through two nuclei.

This is indeed necessary, since there non-universal phenomena occur. Also, the order $\mathcal{O}(1 / \sqrt{E})$ of aperture is optimal, as we show now by example.

We consider the purely Coulombic two-centre problem (see Appendix B) with $\vec{s}_{1}:=\left(\begin{array}{l}1 \\ 0 \\ 0\end{array}\right), \vec{s}_{2}:=\left(\begin{array}{c}-1 \\ 0 \\ 0\end{array}\right), Z_{1}>0$ and $Z_{2}=-Z_{1}$.

We claim that for energy $E>E_{\text {th }}$ there is no orbit colliding with $\vec{s}_{1}$ and having scattering angles $\theta(\infty)$ w.r.t. to the negative 1 -axis smaller than $\frac{1}{2} \sqrt{\left|Z_{2}\right| / E}$, whereas there are such orbits for $\theta(\infty)>2 \sqrt{\left|Z_{2}\right| / E}$.

Such an orbit would lie in a plane containing $\vec{s}_{1}$ and $\vec{s}_{2}$, say the $1-2$-plane. By reflection symmetry we need only consider the part of the orbit after collision, and we denote by $\tau$ the time of its last intersection with the $q_{1} \equiv 0-$ plane. Then $V(\vec{q}(\tau))=0$ so that $p_{1}(\tau)=-\sqrt{2\left(E-p_{2}^{2}(\tau)\right)}$. So at time $\tau$ the angle of the particle direction $\vec{p}(\tau)$ with the negative 1 -axis equals

$$
\theta(\tau)=\arcsin \left(\left|p_{2}(\tau)\right| / \sqrt{2 E}\right)
$$

As we are interested in small scattering angles, we may assume $\left|p_{1}(\tau)\right|>\left|p_{2}(\tau)\right|$. Moreover

$$
q_{2}(\tau)=\frac{p_{2}(\tau)}{-p_{1}(\tau)}+\mathcal{O}(1 / E)
$$

as follows from the estimates of Sect. 9. Thus the angular momentum w.r.t. $\vec{s}_{2}$ equals

$$
\left|\vec{L}_{2}\right|=\left|\left(\vec{q}(\tau)-\vec{s}_{2}\right) \times \vec{p}(\tau)\right|=\left|p_{2}(\tau)-q_{2}(\tau) p_{1}(\tau)\right|=2\left|p_{2}(\tau)\right|+\mathcal{O}(1 / E) .
$$

As the scattering angle $\Delta \theta$ in a Coulombic potential of charge $Z_{2}$ meets the relation

$$
\frac{\sin \Delta \theta}{1+\cos \Delta \theta}=\frac{\left|Z_{2}\right|}{\sqrt{2 E}\left|\vec{L}_{2}\right|}
$$

we obtain for $1 / \sqrt{E} \ll\left|p_{2}(\tau)\right| \ll \sqrt{E}$ the total scattering angle

$$
\theta(\infty)=\theta(\tau)+\Delta \theta \cdot(1+o(1))=(2 E)^{-1 / 2}\left[\left|p_{2}\right|+\frac{\left|Z_{2}\right|}{\left|p_{2}\right|}\right] \cdot(1+o(1))
$$

which is minimized by $\left|p_{2}\right| \approx \sqrt{\left|Z_{2}\right|}$, with value

$$
\theta(\infty)=\sqrt{2\left|Z_{2}\right| / E} \cdot(1+o(1))
$$


Proof of Theorem 15.3. As remarked before Lemma 8.4, there are two regimes for Coulomb scattering, hard and soft scattering. A large choice of the constant $C_{7}$ in Theorem 15.3 separates the two regimes.

1) In order to prove the theorem, we thus first have to fix the constants $c_{1}, c_{2}$ which appear in Part 1) and 2) of Lemma 8.4, for $\delta=\frac{1}{2}$. The lemma describes the linearization of the Kepler Transformation, but its estimates are also valid for the motion in the potential $V$, using estimate (8.20) and (8.21).

We are interested in the effect of that linearized scattering transformation on cone fields of the form

$$
\mathcal{C}(a):=\left\{\left(\begin{array}{l}
\delta \vec{u} \\
\delta \vec{v}
\end{array}\right)|| \delta \vec{u}-\delta \vec{v}|\leq a| \delta \vec{u}+\delta \vec{v} \mid\right\} \quad(a>0) .
$$

So we have $\mathcal{C}(b) \subset \mathcal{C}(a)$ for $b<a$ (note for comparison that in (11.1) we used the cone field with energy-dependent $a=C / E$ ).

- $\varepsilon>0$ is chosen small enough so that the (according to Lemma 7.1 nearly free) linearized flow within $\mathcal{I Z}\left(c_{q}\right)$ maps the cone $\mathcal{C}(1+2 \varepsilon)$ into $\mathcal{C}(2)$, and the cone $\mathcal{C}\left(\frac{1}{2}\right)$ into $\mathcal{C}(1-2 \varepsilon)$.

For such a small $\varepsilon>0$ we then choose

- $c_{1}>0$ large enough so that the linearized scattering transformation maps the cone $\mathcal{C}(2)$ into $\mathcal{C}\left(\frac{1}{2}\right)$

- $c_{2}>0$ small enough so that the linearized scattering transformation maps the cone $\mathcal{C}(1+\varepsilon)$ into $\mathcal{C}(1+2 \varepsilon)$.

All these choices can indeed be made (apply the relevant unperturbed matrices $\left(\begin{array}{ll}R & R \\ R & R\end{array}\right)$ and $\left(\begin{array}{cc}11 & 0 \\ s & \mathbb{1}\end{array}\right)$ from (8.9) and (7.2), resp. (8.10) on the vector $\left.\left(\begin{array}{l}\delta \vec{u} \\ \delta \vec{v}\end{array}\right)\right)$.

By fixing the constants $c_{1}$ and $c_{2}$, we have defined soft and hard scattering.

2) Now all $\theta$-visits in the sense of Definition 12.6 (meaning that the orbit of energy $E$ locally intersects the Poincaré surface $\left.\mathcal{H}_{l}(\theta(E))\right)$, are hard scattering events.

Namely, as $\theta(E)=4 c_{1} / \sqrt{E}$ (see (15.2)), the scattering angle $\Delta \psi$ inside the ball $B_{l}\left(c_{q}\right)$ is bounded below by

$$
\Delta \psi \geq \theta(E) / 4=c_{1} / \sqrt{E},
$$

using (9.3). It thus meets the criterion of Lemma 8.4 for hard scattering.

3) We show now that, for large enough $C_{7}$ in (15.1) (i.e. by excluding large cones of asymptotic directions $\hat{p}^{ \pm}(x)$ around the axes through two centres), Diff $E$ is well-defined, that is, that every scattering state $x \in s_{E}(\vartheta(E)) \theta$-visits at least one nucleus, so that $\mathcal{N C T}_{\theta}(x) \neq \varnothing$.

In view of Lemma 15.2 the orbit through $x \in s_{E}(\vartheta(E))$ meets the interaction zone. If the directions at the times $T_{E}^{ \pm}(x)$ of entrance resp. exit are denoted by $\hat{p}_{i}$ resp. $\hat{p}_{o}$, then like in (15.3), we have the estimate

$$
\varangle\left(\hat{p}_{i}, \hat{p}^{-}(x)\right)+\varangle\left(\hat{p}_{o}, \hat{p}^{+}(x)\right)=\mathcal{O}(1 / E) \quad\left(E>E_{\mathrm{th}}\right) .
$$

Since $\varangle\left(\hat{p}^{-}(x), \hat{p}^{+}(x)\right)>\vartheta(E)$, we conclude that for large enough $C_{7}$ the change of direction inside the interaction zone is at least

$$
\varangle\left(\hat{p}_{i}, \hat{p}_{o}\right)>\frac{1}{2} \vartheta(E) .
$$


Lemma 7.1 then tells us that the trajectory enters at least one ball of (energyindependent) radius $c_{q}$ around a singularity $\vec{s}_{l}$.

We count the number $N$ of such occurrences. If $N \geq 3$, then Proposition 9.2 says that the orbit $\alpha_{\min } / 2$-visits at least one nucleus. Comparing with the definition (15.2) of $\theta$, this is a $\theta$-visit.

But the same holds for $N \leq 2$, since otherwise

- the changes of directions inside the $N c_{q}$-balls are each smaller than $\theta$ (Lemma 9.1), and

- the $N+1$ components of the trajectory inside the interaction zone but outside the $c_{q}$-balls each contribute at most with $C / E$ to the change of direction (Lemma 7.1).

- Adding these contributions and using the definition (15.1) of $\theta$, we would get

$$
\varangle\left(\hat{p}_{i}, \hat{p}_{o}\right)<\frac{3 C}{E}+\theta(E)=\frac{3 C}{E}+\frac{C_{9}}{\sqrt{E}}<\frac{1}{2} \frac{C_{7}}{\sqrt{E}}=\frac{1}{2} \vartheta(E) \quad \text { for } E_{\text {th }} \text { large, }
$$

in contradiction with (15.5) if

$$
C_{7} \geq 4 C_{9}
$$

4) We want to avoid intermediate scattering events with angles $\Delta \Psi$ meeting

$$
\frac{c_{2}}{\sqrt{E}} \leq \Delta \Psi \leq \frac{c_{1}}{\sqrt{E}}
$$

This can be done, too, by choosing $C_{7}$ in (15.1) large enough.

From 3) we know that the orbit $\theta$-visits the centres in some succession $\underline{k}=\left(k_{1}, \ldots, k_{r}\right)$. There can be at most one visit of some ball $B_{k_{0}}\left(c_{q}\right)$ before and some ball $B_{k_{r+1}}\left(c_{q}\right)$ after this sequence of near-collisions (Proposition 9.2).

Moreover, for large $C_{7}$, these visits, if they occur, are soft scattering events in the sense of Lemma 8.4. We need only prove this for $B_{k_{0}}\left(c_{q}\right)$, as the result for $B_{k_{r+1}}\left(c_{q}\right)$ then follows by time reversal.

So we consider the half-orbit $\Phi\left(\left(-\infty, t_{1}\right], x\right)$ through $x$ with $t_{1}=\min \mathcal{N C T}_{\theta}(x)$, see Definition 12.6. This half-orbit ends in the Poincaré surface near $\vec{s}_{k_{1}}$, namely $\Phi\left(t_{1}, x\right) \in \mathcal{H}_{k_{1}}(\theta)$. More precisely, by def. (8.1) the configuration space distance is bounded by

$$
\left|\vec{q}\left(t_{1}, x\right)-\vec{s}_{k_{1}}\right| \leq \frac{4 Z_{\max }}{C_{9} \sqrt{E}}
$$

The half-trajectory $\vec{q}\left(\left(-\infty, t_{1}\right], x\right)$ consists of three types of segments:

1. The segment outside the interaction zone $\mathcal{I Z}$. Here the total change of direction is of order $\mathcal{O}(1 / E)$ (Theorem 6.3).

2. The (one or two) segments in $\mathcal{I Z}\left(c_{q}\right)$. Here, too the total change of direction is of order $\mathcal{O}(1 / E)($ Lemma 7.1). 
3. The segment inside the ball $B_{k_{0}}\left(c_{q}\right)$, perhaps empty. We know that the halforbit has no hard collision here, i.e. it misses $\mathcal{H}_{k_{0}}(\theta)$ (otherwise $\underline{k}$ would begin with $k_{0}$ instead of $\left.k_{1}\right)$ ). Thus the total change of direction inside $B_{k_{0}}\left(c_{q}\right)$ is bounded by

$$
\Delta \psi<\theta=\frac{C_{9}}{\sqrt{E}} .
$$

Summing these contributions, for $E_{\text {th }}$ large the total change of direction on the half-orbit is bounded by

$$
\varangle\left(\hat{p}^{-}(x), \vec{p}(t)\right)<\frac{2 C_{9}}{\sqrt{E}} \quad(-\infty<t \leq \tau),
$$

$\tau$ being the time where the half orbit enters the ball $B_{k_{1}}\left(c_{q}\right)$

On the other hand by our assumption $x \in s_{E}(\vartheta(E))$

$$
\varangle\left(\hat{p}^{-}(x), \hat{s}^{k_{1}, k_{0}}\right)>\frac{C_{7}}{\sqrt{E}} .
$$

$\left(\vec{v}^{-}, \vec{w}^{-}\right)=\left(\vec{p}(\tau, x) / \sqrt{2 E},\left(\vec{q}(\tau, x)-\vec{s}_{k_{1}}\right) / c_{q}\right)$ are the coordinates $(8.3)$ of the end point $\Phi(\tau, x)$ of the half-orbit. By Lemma 8.2

$$
\varangle\left(\vec{v}^{-},-\vec{w}^{-}\right)<\frac{\pi \cdot E_{\mathrm{th}}}{\frac{1}{4} C_{9} \sqrt{E}},
$$

so that with (15.7) the relative position $\vec{w}^{-}$meets

$$
\varangle\left(\vec{w}^{-},-\hat{p}^{-}(x)\right) \leq \varangle\left(\hat{p}^{-}(x), \vec{v}^{-}\right)+\varangle\left(\vec{v}^{-},-\vec{w}^{-}\right)<\left(2 C_{9}+\frac{4 \pi \cdot E_{\mathrm{th}}}{C_{9}}\right) / \sqrt{E} .
$$

Together with (15.7) and (15.8) this means that for $C_{7}$ large the distance of $\vec{q}(t, x)$ from the line $L$ through $\vec{s}_{k_{0}}$ and $\vec{s}_{k_{1}}$ is bounded by

$$
\operatorname{dist}(\vec{q}(t, x), L) \geq \frac{1}{2} \frac{C_{7}}{\sqrt{E}} \cdot\left|\vec{q}(t, x)-\vec{s}_{k_{1}}\right| \quad(-\infty<t \leq \tau) .
$$

But then

$$
\operatorname{dist}\left(\vec{q}(t, x), \vec{s}_{k_{0}}\right) \geq \frac{1}{2} \frac{C_{7}}{\sqrt{E}} \cdot d_{\min } / 2 \quad(-\infty<t \leq \tau) .
$$

so that there is at most soft scattering inside $B_{k_{0}}\left(c_{q}\right)$, if $C_{7}$ is large. Thus there are no intermediate scattering events with angles meeting (15.6).

5) The last remark implies that the symbolic sequence $x \mapsto w(x)$ is locally constant on $s_{E}(\vartheta(E))$. So by Lemma 15.2 and Theorem 6.5 the map Diff $E$ is smooth.

6) Our next task is to show that $\operatorname{Diff}_{E}$ is onto. It suffices to find for given data

$$
\left(\hat{\theta}^{-}, \hat{\theta}^{+}, \underline{k}\right) \in \mathcal{A D}(\vartheta) \times \mathcal{W}
$$

an orbit in $\Sigma_{E}$ with these asymptotic directions and $\theta$-visits. 
We proceed in a way similar to the construction of bounded orbits in Sects. 11 and 12 . If the symbol sequence equals $\underline{k}=\left(k_{1}, \ldots, k_{r}\right)$, then we erect incoming resp. outgoing Poincaré surfaces $V\left(\hat{\theta}^{-}, k_{1}\right)$ resp. $W\left(k_{r}, \hat{\theta}^{+}\right)$with

$$
\begin{aligned}
& V(\hat{\theta}, l):=\left\{x \equiv(\vec{p}, \vec{q}) \in \mathcal{D}|| \vec{q}-\vec{s}_{l} \mid=c_{q}, \frac{\left|\vec{q}-\vec{q}_{i}\right|}{c_{q}}<\frac{C_{10}}{\sqrt{H(x)}},\right. \\
& \left.\left|\frac{\vec{p}}{\sqrt{2 H(x)}} \times \hat{\theta}\right|<\frac{2 C_{10}}{\sqrt{H(x)}}\right\}, \\
& W(l, \hat{\theta}):=\left\{x \equiv(\vec{p}, \vec{q}) \in \mathcal{D}|| \vec{q}-\vec{s}_{l} \mid=c_{q}, \frac{\left|\vec{q}-\vec{q}_{i}\right|}{c_{q}}<\frac{C_{10}}{\sqrt{H(x)}},\right. \\
& \left.\left|\frac{\vec{p}}{\sqrt{2 H(x)}} \times \hat{\theta}\right|<\frac{2 C_{10}}{\sqrt{H(x)}}\right\},
\end{aligned}
$$

$\hat{\theta} \in S^{2}$, near $\vec{q}_{i}:=\vec{s}_{l}-c_{q} \hat{\theta}$ resp. near $\vec{q}_{o}:=\vec{s}_{l}+c_{q} \hat{\theta}$ (compare this definition with the one of $\mathcal{H}_{E}^{k, l}$ in (9.16)).

For all $\vec{q}^{-}$in the configuration space projection of $V\left(\hat{\theta}^{-}, k_{1}\right)$ and all $\vec{q}^{+}$in the configuration space projection of $W\left(k_{r}, \hat{\theta}^{+}\right)$there exist $\vec{p}^{ \pm}$with $\left(\vec{p}^{-}, \vec{q}^{-}\right) \in$ $V\left(\hat{\theta}^{-}, k_{1}\right),\left(\vec{p}^{+}, \vec{q}^{+}\right) \in W\left(k_{r}, \hat{\theta}^{+}\right)$such that the orbit through $\left(\vec{p}^{-}, \vec{q}^{-}\right) \theta$-visits the centres in succession $\underline{k}$ and then meets $\left(\vec{p}^{+}, \vec{q}^{+}\right)$. The proof of this assertion uses the estimates of Sect. 11 , slightly modifying Proposition 11.5 .

We set $C_{10}:=C_{7} / 8$ and choose a large value of $C_{7}$. As $\Theta_{\min }\left(\hat{\theta}^{ \pm}\right)>\vartheta(E)=$ $C_{7} / \sqrt{E}$, by what we have proven in part 4$), V\left(\hat{\theta}^{-}, k_{1}\right) \operatorname{did}$ not $\theta$-visit a nucleus in the past, and the orbits through $W\left(k_{r}, \hat{\theta}^{+}\right)$will not $\theta$-visit a nucleus in the future. Instead by Proposition 9.2, they leave the interaction zone in time $\mp \mathcal{O}(\sqrt{E})$.

Using estimate (15.7 with $t:=\tau$, we see that for every $\vec{q}^{-}$there is at least one $\vec{p}^{-}$such that the orbit through $\left(\vec{p}^{-}, \vec{q}^{-}\right) \in V\left(\hat{\theta}^{-}, k_{1}\right)$ has asyptotic direction

$$
\lim _{t \rightarrow-\infty} \hat{p}\left(t,\left(\vec{p}^{-}, \vec{q}^{-}\right)\right)=\hat{\theta}^{-} .
$$

By standard arguments this family of orbits contains one $\theta$-visiting the centres in succession $\underline{k}$ and then having limit

$$
\lim _{t \rightarrow \infty} \hat{p}\left(t,\left(\vec{p}^{-}, \vec{q}^{-}\right)\right)=\hat{\theta}^{+}
$$

So $\operatorname{Diff}_{E}$ is onto.

7) Cone field estimates based on Part 1) now show that $\operatorname{Diff}_{E}$ is one to one and smoothly invertible. Specifically we show that there exist invariant cone fields $\mathcal{C}(a)$ (see (15.4)) along the orbits constructed in Part 6).

The idea is simply that there is at least one hard scattering along the scattering orbit, making the family of energy $E$ configuration space trajectories with initial asymptotic direction $\hat{\theta}^{-}$divergent for large positive times.

So for given $\hat{\theta}^{-}$we consider the Lagrange manifold of points $x \in s_{E}$ having asymptotic direction $\hat{p}^{-}(x)=\hat{\theta}^{-}$. We claim that for $E_{\text {th }}$ large the tangent space of this submanifold at $\left(\vec{p}^{-}, \vec{q}^{-}\right) \in V\left(\hat{\theta}^{-}, k_{1}\right)$ is contained in the (wide) cone $\mathcal{C}(2)$. Namely the half-orbit ending in $\left(\vec{p}^{-}, \vec{q}^{-}\right)$consist of at most four segments: 
1. The segment outside the interaction zone $\mathcal{I Z}$, controlled by Theorem 6.3. For large $E_{\text {th }}$ this is in a cone $\mathcal{C}(1+\varepsilon / 2)$.

2. The segment in $\mathcal{I Z}\left(c_{q}\right)$ before entering the ball $B_{k_{0}}\left(c_{q}\right)$, controlled by Lemma 7.1. Here the linearized Lagrange manifold is contained in $\mathcal{C}(1+\varepsilon)$.

3. At most one segment in $B_{k_{0}}\left(c_{q}\right)$, controlled by the choice of the soft scattering constant $c_{2}$ in Part 1) of this proof. So after this soft collision, we are in $\mathcal{C}(1+2 \varepsilon)$.

4. The segment in $\mathcal{I Z}\left(c_{q}\right)$ after leaving $B_{k_{0}}\left(c_{q}\right)$ and before entering $B_{k_{1}}\left(c_{q}\right)$. By Part 1) here the linearized Lagrange manifold is contained in $\mathcal{C}(2)$.

Our choice of the hard scattering constant $c_{1}$ implies that after leaving $B_{k_{1}}\left(c_{q}\right)$ we are in the narrow cone $\mathcal{C}\left(\frac{1}{2}\right)$. At least the same is true after leaving $B_{k_{r}}\left(c_{q}\right)$, using the cone field estimates of Sect. 11. Finally, we consider the positive half orbit starting at $\left(\vec{p}^{+}, \vec{q}^{+}\right) \in W\left(k_{r}, \hat{\theta}^{+}\right)$and get a similar sequence

$$
\mathcal{C}\left(\frac{1}{2}\right) \rightarrow \mathcal{C}(1-2 \varepsilon) \rightarrow \mathcal{C}(1-\varepsilon) \rightarrow \mathcal{C}\left(1-\frac{1}{2} \varepsilon\right)
$$

of cone fields, using Part 1) again. For large energies this shows uniqueness of the orbit with data (15.9).

\section{The differential cross section}

The scattering transformation $S$ defined in (6.15) contains complete information on the scattering process. As we have seen in the previous sections, it exhibits many aspects of irregularity if $n \geq 3$. Nevertheless, the scattering transformation is not directly accessible in a (classical) scattering experiment.

Firstly, one typically cannot fix the initial angular momentum of the test particle. Secondly (unlike in a quantum mechanical setting where interference effects exist) it is hard to measure time delay.

What is accessible is the differential cross section $\frac{d \sigma}{d \hat{\theta}^{+}}\left(E, \hat{\theta}^{-}, \hat{\theta}^{+}\right)$. Informally speaking, this is the (density of the) number of particles per second scattered in the final direction $\hat{\theta}^{+}$, assuming a uniform flux of one particle per second and unit area of incoming particles of energy $E$ and initial direction $\hat{\theta}^{-}$.

One could expect to see some trace of irregularity in the differential cross section, and in fact for all systems considered up to now numerical calculations of the cross section indicated the existence of so-called rainbow singularities on a Cantor set of angles, see [Ec,EJ,Ga,Sm,Te].

For the simplest case of a Kepler potential we obtain the so-called Rutherford cross section, see (16.6) below. It is remarkable that the differential cross sections for the cases $n=2$ and $n \geq 3$ turn out to be very similar to the Rutherford cross section (see (16.8) for the statement, and Fig. 12.1 of [KK] for a numerical plot for $d=2$ dimensions). So the complicated structure of the time delay and the scattering orbits is not reflected in the cross section.

The reason for that discrepancy is, roughly speaking, the following. For $d=2$ the deflection functions $L^{-} \mapsto \hat{\theta}^{+}\left(E, \hat{\theta}^{-}, L^{-}\right)$(depicted in [KK], Figs. 10.2-10.3) are strictly monotonic w.r.t. the initial angular momentum $L^{-}$. It is clear from the 
definition of $\frac{d \sigma}{d \hat{\theta}^{+}}$that extrema of the deflection function lead to singularities in the differential cross section. Since there are no extrema (except for the degenerate situation $L^{-} \rightarrow \pm \infty$ ), we have a nonsingular $\frac{d \sigma}{d \hat{\theta}^{+}}$(except for the forward direction).

Before stating our theorems, we shall recall a mathematically correct definition of cross section. In the physics literature the cross section is sometimes introduced as a function, whereas it really is a measure. The difference is of some importance because in general that cross section measure is not absolutely continuous w.r.t. Haar measure. In our context, we shall show that under certain conditions the cross section measure is absolutely continuous if one excludes the forward direction and the directions near $\hat{s}^{k, l}$, and that the Radon-Nikodym derivative, i.e. the differential cross section, is smooth.

We denote by

$$
\vec{a}: P_{+} \rightarrow \mathbb{R}^{3} \quad, \quad(\vec{p}, \vec{q}) \mapsto \vec{q}-\langle\vec{q}, \hat{p}\rangle \hat{p}
$$

the impact parameter. For phase space points $x \in P_{+}$projecting to a singularity $\vec{s}_{l}$ or with $x \equiv(\vec{p}, \vec{q})=(\overrightarrow{0}, \vec{q})$ the impact parameter is defined by, say $\vec{a}(x):=\overrightarrow{0}$.

Now on the \pm -scattering states $s^{ \pm} \subset \vec{P}_{+}$the asymptotic impact parameters

$$
\vec{a}^{ \pm}: s^{ \pm} \rightarrow \mathbb{R}^{3} \quad, \quad \vec{a}^{ \pm}:=\lim _{t \rightarrow \pm \infty} \vec{a} \circ \Phi^{t}
$$

are well-defined continuous functions, since $\vec{a}(\vec{p}, \vec{q})$ is continuous outside the interaction zone (that is, for $|\vec{q}| \geq R_{\mathrm{vir}}(H(\vec{p}, \vec{q}))$ ), and there

$$
\vec{a}(\vec{p}, \vec{q})=\frac{\vec{p} \times \vec{L}(\vec{p}, \vec{q})}{2(H(\vec{p}, \vec{q})-V(\vec{p}, \vec{q}))} .
$$

By Theorem 6.3 the r.h.s. of (16.1) has a limit on scattering orbits.

For energy $E>0$ we consider the maps

$$
A_{E}^{ \pm}: s_{E} \rightarrow T^{*} S^{2} \quad, \quad x \mapsto\left(\vec{a}^{ \pm}(x), \hat{p}^{ \pm}(x)\right)
$$

from the set of \pm scattering states of energy $E$ to their asymptotic data. These can really be considered as points in the cotangent bundle

$$
N:=T^{*} S^{2}
$$

of the two-sphere, since

- $\left|\hat{p}^{ \pm}(x)\right|=1$,

- $\left\langle\vec{a}^{ \pm}(x), \hat{p}^{ \pm}(x)\right\rangle=0$, and

- the bilinear map $(\vec{p}, \vec{q}) \mapsto\langle\vec{p}, \vec{q}\rangle$ is the natural pairing between vectors and co-vectors (no Riemannian metric involved).

The cotangent bundle $N$ carries the canonical symplectic two-form $\omega_{N}$ and the volume form $\lambda_{N}:=\frac{1}{2} \omega_{N} \wedge \omega_{N}$.

The maps $A_{E}^{ \pm}$are constant on orbits, and for $y \in N$ the preimages $\left(A_{E}^{ \pm}\right)^{-1}(y)$ consist of at most one orbit. 
Proposition 16.1. For energy $E>0$ the set $A_{E}^{ \pm}\left(s_{E}\right) \subset N$ of asymptotic data of the scattering states is open, and its complement $N \backslash A_{E}^{ \pm}\left(s_{E}\right)$ is a compact set of $\lambda_{N}$-measure zero.

If $V$ satisfies the decay estimates (6.16), then the map

$$
M_{E}: A_{E}^{-}\left(s_{E}\right) \rightarrow A_{E}^{+}\left(s_{E}\right) \quad, \quad A_{E}^{-}(x) \mapsto A_{E}^{+}(x) \quad\left(x \in s_{E}\right)
$$

from initial to final data for energy $E$ is a smooth canonical transformation.

Proof. For energy $E>0$ and radius $r>R_{\mathrm{vir}}(E)$ we consider the smooth Poincaré surfaces

$$
U_{E, r}^{ \pm}:=\left\{(\vec{p}, \vec{q}) \in \Sigma_{E}|| \vec{q} \mid=r, \pm\langle\vec{p}, \vec{q}\rangle>0\right\} .
$$

These four-manifolds are transversal to the flow as $\left\{H, \vec{q}^{2}\right\}=\frac{1}{2}\left\{\vec{p}^{2}, \vec{q}^{2}\right\}=$ $-2\langle\vec{p}, \vec{q}\rangle \neq 0$. According to Lemma 8.2 of McDuff and Salamon [DS], they are symplectic submanifolds of our phase space $P$, and the Poincaré section map

$$
U_{E, r}^{-} \cap s_{E} \rightarrow U_{E, r}^{+} \cap s_{E}
$$

which send $x$ to the unique intersection point of the orbit $\Phi(\mathbb{R}, x)$ with $U_{E, r}^{+}$is a symplectomorphism.

We use on $U_{E, r}^{ \pm}$the coordinates $(\vec{a}, \hat{p})$, which are maps

$$
\kappa_{E, r}^{ \pm}: U_{E, r}^{ \pm} \rightarrow N
$$

Indeed they are diffeomorphisms onto their common image

$$
N_{r}:=\left\{\left(\vec{a}^{\prime}, \hat{p}\right) \in N|| \vec{a}^{\prime} \mid<r\right\} .
$$

Thus the map (16.3) induces a diffeomorphism

$$
M_{E, r}: N_{r} \cap \kappa_{E, r}^{-}\left(s_{E}\right) \rightarrow N_{r} \cap \kappa_{E, r}^{+}\left(s_{E}\right)
$$

which converges pointwisely to (16.2) as $r \rightarrow \infty$, using Theorem 6.3. With assumption (6.16), smoothness of $M_{E}$ is then implied by Theorem 6.5.

The scattering states $s \subset P$ form an open subset (see Thm 2.3.3 of [DG]), and thus $U_{E, r}^{-} \cap s_{E}$ is relatively open, too. All orbits in $\Sigma_{E}$ which do not meet the interaction zone $\mathcal{I Z}(E)$ are scattering. Thus $N \backslash A_{E}^{ \pm}\left(s_{E}\right)$ is a compact set. Then using $A_{E}^{ \pm}\left(s_{E}^{ \pm}\right)=N$ and asymptotic completeness Corollary 6.4.2, we see that $\lambda_{N}\left(N \backslash A_{E}^{ \pm}\left(s_{E}\right)\right)=0$.

For $\lambda_{S^{2}}$-almost all $\hat{\theta}^{-} \in S^{2}$ the map

$$
\hat{\varphi}_{E, \hat{\theta}^{-}}: T_{\hat{\theta}^{-}}^{*} S^{2} \rightarrow S^{2}, \quad \hat{\varphi}_{E, \hat{\theta}^{-}}\left(\vec{a}^{-}\right):=\hat{p}^{+}\left(E, \vec{a}^{-}, \hat{\theta}^{-}\right)
$$

is measurable. Here the restriction of the asymptotic direction $\hat{p}^{+}: s^{+} \rightarrow S^{2}$ (which is constant on orbits) to $s_{E}$ is considered as a map

$$
\hat{p}^{+}(E, \cdot, \cdot): A_{E}^{-}\left(s_{E}\right) \rightarrow S^{2},
$$

see Proposition 16.1. 
Definition 16.2. For $E>0$ and $\hat{\theta}^{-} \in S^{2}$ the cross section measure $\sigma\left(E, \hat{\theta}^{-}\right)$on $S^{2}$ is the image measure

$$
\sigma\left(E, \hat{\theta}^{-}\right):=\hat{\varphi}_{E, \hat{\theta}^{-}}\left(\lambda_{\hat{\theta}^{-}}\right)
$$

$\lambda_{\hat{\theta}^{-}}$being Lebesgue measure on the cotangent plane $T_{\hat{\theta}^{-}}^{*} S^{2}$.

Assuming $\sigma\left(E, \hat{\theta}^{-}\right)$on $S^{2} \backslash\left\{\hat{\theta}^{-}\right\}$to be absolutely continuous w.r.t. Haar measure $\lambda_{S^{2}}$, the differential cross section $\frac{d \sigma}{d \hat{\theta}^{+}}\left(E, \hat{\theta}^{-}, \hat{\theta}^{+}\right)$is the Radon-Nikodym derivative of $\sigma\left(E, \hat{\theta}^{-}\right)$.

In Definition (16.5) we by using $\lambda_{\hat{\theta}^{-}}$we normalize the flux through unit area in configuration space to equal one.

Example 16.3. By radial symmetry, for the simplest case (6.1) of scattering by a Kepler potential with $Z \equiv Z_{\infty} \neq 0$, the (Rutherford) differential cross section depends only on the angle

$$
\Delta \theta:=\varangle\left(\hat{\theta}^{-}, \hat{\theta}^{+}\right)
$$

between the initial and final direction. Using formula (8.12) for the eccentricity $e=+1 / \sin \left(\frac{1}{2} \Delta \theta\right)$ of the Kepler hyperbola, we see that the modulus $a$ of the impact parameters $\vec{a}^{ \pm}$equals

$$
a=\frac{|Z|}{2 E} \cot \left(\frac{1}{2} \Delta \theta\right),
$$

so that

$$
\left|\frac{d a}{d \Delta \theta}\right|=\frac{|Z|}{4 E \sin ^{2}\left(\frac{1}{2} \Delta \theta\right)} .
$$

We may assume that $\hat{\theta}^{-}=(0,0,1)$ so that the 3 -component of $\vec{a}^{-}=\vec{p}^{-} \times \vec{L}^{-}$ vanishes and $\lambda_{\hat{\theta}^{-}}$corresponds to integration with the two-form $d L_{1}^{-} \wedge d L_{2}^{-}$. Introducing polar coordinates $\left(L, \varphi^{-}\right)$in the plane $T_{\hat{\theta}^{-}}^{*} S^{2}$, and expressing the volume element at $\hat{\theta}^{+}$on $S^{2}$ in the form

$$
\sin (\Delta \theta) \cdot d \varphi^{-} \wedge d \Delta \theta,
$$

we obtain the familiar expression

$$
\left(\frac{d \sigma}{d \hat{\theta}^{+}}\left(E, \hat{\theta}^{-}, \hat{\theta}^{+}\right)\right)_{\mathrm{Ru}}=\left|\frac{L}{2 E} \frac{d L}{d \Delta \theta}\right|=\left(\frac{Z}{4 E \sin ^{2}\left(\frac{1}{2} \Delta \theta\right)}\right)^{2}
$$

for the Rutherford cross section. Note that it depends only on the modulus of the charge $Z$.

$$
\begin{aligned}
\text { For }\left(\hat{\theta}^{-}, \hat{\theta}^{+}\right) \in & \Theta_{\text {min }}^{-1}(\theta), \underline{k} \in \mathcal{W} \text { let } \\
& \vec{a}_{\underline{k}}^{-}\left(E, \hat{\theta}^{-}, \hat{\theta}^{+}\right):=\lim _{t \rightarrow-\infty} \vec{a}\left(\operatorname{Diff}_{E}^{-1}\left(t, \hat{\theta}^{-}, \hat{\theta}^{+}, \underline{k}\right),\right.
\end{aligned}
$$

with $\operatorname{Diff}_{E}$ defined in Theorem 15.3. 
Theorem 16.4. Let $V$ be a Coulombic potential satisfying the decay estimates (6.16).

Then for energy $E>E_{\text {th }}$ and $\vartheta=\min \left(c E^{-\delta}, \alpha_{\min }\right)$ with $0 \leq \delta \leq \frac{1}{2}$, on $\Theta_{\min }^{-1}(\vartheta)$ the differential cross section is smooth, of the form

$$
\frac{d \sigma}{d \hat{\theta}^{+}}\left(E, \hat{\theta}^{-}, \hat{\theta}^{+}\right)=\sum_{\underline{k} \in \mathcal{W}}\left|\operatorname{det}\left(\frac{d \hat{\varphi}_{E, \hat{\theta}^{-}}}{d \vec{a}}\left(\vec{a}_{\underline{k}}^{-}\left(E, \hat{\theta}^{-}, \hat{\theta}^{+}\right)\right)\right)\right|^{-1}
$$

and differs from the Rutherford cross section (16.6) for charge $Z:=\sqrt{\sum_{l=1}^{n} Z_{l}^{2}}$ only by

$$
\frac{d \sigma}{d \hat{\theta}^{+}}\left(E, \hat{\theta}^{-}, \hat{\theta}^{+}\right)=\left(\frac{d \sigma}{d \hat{\theta}^{+}}\left(E, \hat{\theta}^{-}, \hat{\theta}^{+}\right)\right)_{\mathrm{Ru}} \cdot\left(1+\mathcal{O}\left(E^{2 \delta-1}\right)\right)
$$

uniformly in $\left(\hat{\theta}^{-}, \hat{\theta}^{+}\right) \in \Theta_{\min }^{-1}(\vartheta)$.

Remarks 16.5. 1) In particular the relative difference w.r.t. Rutherford cross section is only of order $\mathcal{O}(1 / E)$ if one excludes cones of an energy-independent aperture $\vartheta$. 2) In 2-dim. and purely Coulombic potentials the differential cross section is even smooth (up to the forward direction $\hat{\theta}^{+}=\hat{\theta}^{-}$) for all positive energies [KK]. As shown in [Kn3], this smoothness is rather exceptional.

Also here for $n>1$ centres we cannot have smoothness of the differential cross section (for $n o V$ and no $E>E_{\mathrm{th}}$ ), if we add to $\mathcal{A D}(\vartheta)$ the neighbourhood of any direction $\hat{s}^{i, k}$. This follows from the observation that (contrary to the 2D attracting case) in 3 dimensions any hard collision with a nucleus changes the degree. By a limit argument there must then exist points where the degree is zero. At these points the differential cross section diverges.

Proof. Theorem 15.3 says that the orbits with data $\left(E, \hat{\theta}^{-}, \hat{\theta}^{+}\right)$are enumerated by $\mathcal{W}$. So if the r.h.s. of (16.7) converges, then by the Transformation Theorem for Lebesgue measure (16.7) follows from the definition of the cross section measure in (16.5).

Estimates (6.17) and (6.18) of Theorem 6.5 imply that for $\left|\vec{q}_{0}\right| \geq R_{\mathrm{vir}}(E)$, $\pm\left\langle\vec{q}_{0}, \vec{p}_{0}\right\rangle \geq 0$ and multi-indices $\gamma:=(\alpha, \beta) \in \mathbb{N}_{0}^{3} \times \mathbb{N}_{0}^{3}$

$$
\partial_{x_{0}}^{\gamma}\left(\hat{p}^{ \pm}\left(x_{0}\right)-\hat{p}_{0}\right)=\mathcal{O}\left(E^{-1-\frac{1}{2}|\alpha|}\right)
$$

and

$$
\partial_{x_{0}}^{\gamma}\left(\vec{a}^{ \pm}\left(x_{0}\right)-\vec{a}\left(x_{0}\right)\right)=\mathcal{O}\left(E^{-1-\frac{1}{2}|\alpha|}\right),
$$

the last estimate being obtained with the help of (16.1).

Similar statements are true for orbit segments in $\mathcal{I Z}\left(c_{q}\right)$.

So up to an error of order $\mathcal{O}(1 / E)$, all variations of the asymptotic data come from the single scattering processes within the balls of radius $c_{q}$ around the singularities.

We switch to $(\vec{y}, \vec{z})$-coordinates.

There are two types of such contributions: 
1. The ones coming from the hard collisions ( $\theta$-visits in succession $\underline{k})$. These lead to factors of the form (11.2) in the product formula for the linearization:

$$
T_{x} \mathcal{P}_{E}=f\left(k_{-1}, k_{0}, k_{1}\right) E \cdot\left(\begin{array}{ll}
11 & 11 \\
1 & 11
\end{array}\right)+\mathcal{O}\left(E^{0}\right)
$$

with

$$
f\left(k_{-1}, k_{0}, k_{1}\right):=\frac{2 d^{k_{-1}, k_{0}} \cos ^{2}\left(\frac{1}{2} \alpha\left(k_{-1}, k_{0}, k_{1}\right)\right)}{-Z_{k_{0}}} .
$$

As $\Delta \psi>c E^{-\delta}$, the relative error in this estimate is of the order $\mathcal{O}\left(E^{2 \delta-1}\right)$.

2. Visits of $c_{q}$-balls around some singularities, which are not hard collisions ( $\theta$-visits). By what we have shown, there can be at most two such events, one before the $\underline{k}$-visits, one after.

So these visits meet the hypothesis (8.6) of Lemma 8.2

$$
\left|\frac{\vec{v}^{-}}{\left|\vec{v}^{-}\right|}+\vec{w}^{-}\right| \geq c E^{-\delta}
$$

i.e. with angle $\vartheta=\mathcal{O}\left(E^{-\delta^{\prime}}\right)$, where $\delta^{\prime}:=1-\delta \in\left[\frac{1}{2}, 1\right]$.

So formula (8.10) of Lemma 8.4 says that the relative deviation of the linearized flow from free motion during these soft collisions is of order $\mathcal{O}\left(E^{1-2 \delta^{\prime}}\right)=$ $\mathcal{O}\left(E^{2 \delta-1}\right)$ :

$$
\begin{aligned}
& \delta \vec{v}^{+}=\delta \vec{v}^{-}+ \\
& \mathcal{O}\left(c^{2} E^{1-2 \delta^{\prime}}\right) \cdot\left(\left|\delta \vec{v}^{-}\right|+\left|\delta \vec{w}^{-}\right|\right), \\
& \delta \vec{w}^{+}=2 u \delta \vec{v}^{-}+\delta \vec{w}^{-}+2 \vec{v}^{-} d u+\mathcal{O}\left(c^{2} E^{1-2 \delta^{\prime}}\right) \cdot\left(\left|\delta \vec{v}^{-}\right|+\left|\delta \vec{w}^{-}\right|\right) .
\end{aligned}
$$

Now we see that the formal sum (16.7) converges:

- There are exactly $n \cdot(n-1)^{l-1}$ words $\underline{k} \in \mathcal{W}$ of length $l$.

- For word length $l+1$ of $\underline{k} \in \mathcal{W}$ the term

$$
\left|\operatorname{det}\left(\frac{d \hat{\varphi}_{E, \hat{\theta}^{-}}}{d \vec{a}}\left(\vec{a}_{\underline{k}}^{-}\left(E, \hat{\theta}^{-}, \hat{\theta}^{+}\right)\right)\right)\right|
$$

in (16.7) is only of relative order $\mathcal{O}\left(E^{-(d-1)}\right)=\mathcal{O}\left(1 / E^{2}\right)$, compared to the term of the word shortened by one letter, as there is one extra factor $E$ coming from (16.9). Here $d$ denotes the dimension, so $d=3$.

So if $E>E_{\text {th }}$ and the threshold $E_{\text {th }}$ is suitably chosen, this decay outweighs the exponential proliferation of words with given word length.

The comparison in (16.8) with Rutherford cross section (16.6) for squared charge $Z^{2}=\sum_{l=1}^{n} Z_{l}^{2}$ follows by adding the contributions in (16.7) of word length one. In $d=3$ dimensions the leading errors of order $\mathcal{O}\left(E^{2 \delta-1}\right)$ come from 1$)$ and 2 ) above, whereas neglecting the contributions of the longer words is only of order $\mathcal{O}\left(E^{-2}\right)$. 
In $d$ dimensions the Rutherford cross section equals

$$
\left(\frac{d \sigma}{d \hat{\theta}^{+}}\left(E, \hat{\theta}^{-}, \hat{\theta}^{+}\right)\right)_{\mathrm{Ru}}=\left(\frac{|Z|}{4 E \sin ^{2}\left(\frac{1}{2} \Delta \theta\right)}\right)^{d-1} .
$$

In 12.4 of $[\mathrm{KK}]$ we remarked that for $d=2$ dimensions, $Z_{l}>0$ and $\theta^{+} \neq \theta^{-}$ the differential cross section of the $n$-centre problem converges to the $d=2$ Rutherford cross section as $E \rightarrow \infty$. The charge $Z$ in (16.10), however, must be chosen as $Z= \pm \sum_{i=1}^{n} Z_{i}$ (and not $Z_{\infty}$, as wrongly stated in [KK]). This result can be sharpened:

Corollary 16.6. The analog of formula (16.8) holds true in $d=2$ dimensions if one sets $Z:=\sum_{i=1}^{n}\left|Z_{i}\right|$.

Proof. Up to error terms the formulae (11.2) and (8.10) for the linearization of the flow which we used to derive the result (16.8) in $d=3$ are invariant under rotations. Thus (16.8) is true in $d=2$ dimensions, too.

\section{The collinear case}

In this section we show by counterexample that the non-collinearity conditions cannot be dropped in Theorem 12.8 and Theorem 15.3.

1) We first consider the set $b_{E}$ of bounded orbits of energy $E$ for attracting Coulombic potentials $V$ which are rotationally symmetric w.r.t. some axis $A \subset \mathbb{R}_{\vec{q}}^{3}$. Thus in particular the nuclei are situated on that axis:

$$
\vec{s}_{1}, \ldots, \vec{s}_{n} \in A
$$

Conversely, that condition ensures that $V$ is rotationally symmetric around $A$, if it is a purely Coulombic potential.

By symmetry trajectories with initial conditions tangential to a two-plane $F \subset \mathbb{R}_{\vec{q}}^{3}$ containing $A$ stay in $F$, and we may thus consider the restricted twodimensional motion on $F$.

The axis $A$ is divided by $\vec{s}_{1}, \ldots, \vec{s}_{n}$ into $n+1$ closed intervals meeting only in their endpoints $\vec{s}_{l}$. These intervals correspond for energies $E>V_{\max }$ to trajectories which are reflected by the nuclei. Thus two of these trajectories are unbounded and $n-1$ bounded.

These special trajectories are of course invariant under rotations around $A$. However, there cannot be any further energy $E$ trajectories in $F$ having this property.

On the other hand it has been shown in [KK] that for $n \geq 3$ nuclei above some energy $E_{\text {th }}$ there is a Cantor set of bounded trajectories in $F$. So in particular there is an uncountable number of trajectories in $F$ which are not moving tangential to $A$ and thus give rise to one-parameter families of trajectories for the full motion in $\hat{M}$.

2) In general there are bounded orbits which do not lie in any plane $F$ containing the axis $A$. We observe that by rotational symmetry the component $\langle\vec{L}, \hat{s}\rangle$ of 
angular momentum in the direction $\hat{s}$ of $A$ is preserved. We now indicate that for certain collinear configurations there exist two-parameter families of bounded orbits of a given energy, parametrized by that angular momentum component and its conjugate angle.

For the sake of simplicity we consider a 3-centre potential

$$
V(\vec{q}):=-\sum_{l=1}^{3} \frac{Z_{l}}{\left|\vec{q}-\vec{s}_{l}\right|}
$$

with $s_{1}:=\overrightarrow{0}, \vec{s}_{2}:=d \cdot \hat{s}, \vec{s}_{3}:=-d \cdot \hat{s}, \hat{s}:=(0,0,1)$ and $Z_{2}=Z_{3}$ which, in addition of being axially symmetric w.r.t. the 3 -axis $A=\mathbb{R} \cdot \hat{s}$, is mirrorsymmetric w.r.t. reflection by the plane $F_{12}:=\left\{\vec{q} \in \mathbb{R}_{\vec{q}}^{3} \mid q_{3}=0\right\}$. We first consider periodic trajectories with angular momentum component $L_{3}=0$ in, say, the plane $F \cong F_{13}:=\left\{\vec{q} \in \mathbb{R}_{\vec{q}}^{3} \mid q_{2}=0\right\}$ which are invariant under reflection by the plane $F_{12}$ :

$$
q_{1}(-t)=q_{1}(t) \quad, \quad q_{2}(t)=0 \quad, \quad q_{3}(-t)=-q_{3}(t) \quad(t \in \mathbb{R}) .
$$

By symbolic dynamics arguments combined with Theorem 6.11 and Remark 11.2.2 of [KK] for all energies $E>0$ there exists a countable infinity of these orbits, all being hyperbolic and having index 0 (as orbits in the two-plane $F_{13}$. Thus by invoking an implicit function argument one may show the existence of smooth family of energy- $E$ bounded orbits starting on the 1 -axis near $\vec{q}(0)$ and parametrized by $L_{3}$. Rotating these orbits around the axis $A$ then yields a two-parameter family of bounded orbits.

3) For repelling axially symmetric potentials the situation is completely different. W.l.o.g. we again consider potentials $V$ which are invariant w.r.t rotations around the axis $A=\mathbb{R} \cdot \hat{s}$, with $\hat{s}=(0,0,1)$. But now we assume that

$$
\langle\nabla V(\vec{q}), \vec{q}-\langle\vec{q}, \hat{s}\rangle \hat{s}\rangle<0 \quad\left(\vec{q} \in \mathbb{R}_{\vec{q}}^{3} \backslash A\right) .
$$

This condition is met, e.g., by repelling $\left(Z_{l}<0\right)$ purely Coulombic potentials meeting (17.1).

Now consider a trajectory starting at $(\vec{p}(0), \vec{q}(0))$ with

$$
\vec{q}(0)-\langle\vec{q}(0), \hat{s}\rangle \hat{s} \neq \overrightarrow{0} \quad \text { and } \quad\langle\nabla \vec{p}(0), \vec{q}(0)-\langle\vec{q}(0), \hat{s}\rangle \hat{s}\rangle \geq 0 .
$$

Then

$$
\langle\vec{p}(t), \vec{q}(t)-\langle\vec{q}(t), \hat{s}\rangle \hat{s}\rangle>0 \quad(t>0)
$$

and is monotonically inreasing in $t$, since

$$
\frac{d}{d t}\langle\vec{p}, \vec{q}-\langle\vec{q}, \hat{s}\rangle \hat{s}\rangle=-\langle\nabla V(\vec{q}), \vec{q}-\langle\vec{q}, \hat{s}\rangle \hat{s}\rangle+\langle\vec{p}, \vec{p}-\langle\vec{p}, \hat{s}\rangle \hat{s}\rangle>0 .
$$

But this means that the orbit leaves the interaction zone in finite time and thus is not bounded. The second of the conditions in (17.2) is not restrictive, since otherwise we may consider negative times. 
We conclude that the only bounded orbits lie on the axis. Thus for $E>V_{\max }$ there are exactly $n-1$ bounded orbits, compared to the uncountable infinity of bounded orbits in the case of NC configurations and $n \geq 3$.

4) Finally we consider scattering orbits. Already for $n=2$ nuclei and attracting purely Coulombic potentials we have one-parameter families of orbits of a given energy $E$ scattering from a direction parallel to the axis $A$ through the positions $\vec{s}_{1}, \vec{s}_{2}$ and to the backward direction. These are obtained by rotating a given solution around $A$. There are infinitely many such families, as can be seen from the explicit Jacobi solution of the two-centre problem, described in Appendix B, or from [KK], Theorem 12.1 .

\section{A. Aspects of geometry and global analysis}

This article on the 3-dimensional $n$-centre problem is based upon analytical perturbation estimates. To the contrary the 2 -dim. $n$-centre problem (and similarly, the 2-dim. periodic potentials of $[\mathrm{Kn} 1, \mathrm{Kn} 2]$ ) was treated in $[\mathrm{KK}]$ using techniques from Riemannian geometry and global analysis.

In this appendix both approaches are compared.

It is known that the trajectories of energy $E>\sup _{\vec{q}} V(\vec{q})$ generated by a Hamiltonian function $H: T^{*} M \rightarrow \mathbb{R}$ of the (local) form

$$
H(\vec{p}, \vec{q})=\frac{1}{2} \sum_{k, l=1}^{d} g^{k, l}(\vec{q}) p_{k} p_{l}+V(\vec{q})
$$

on a $d$-dimensional Riemannian manifold $(M, g)$ coincide (up to a time reparametrization) with the geodesics in the so-called Jacobi metric $g_{E}$ on $M$, conformally equivalent to $g$ :

$$
g_{E}(\vec{q}):=(1-V(\vec{q}) / E) g(\vec{q})
$$

(here we assume for simplicity $E>0$ ).

In the simplest case covered by the paper $V(\vec{q})=-Z /|\vec{q}|$ with $Z>0$, i.e. the attracting Coulomb potential. There, using a formula from Spivak ([Sp], p. 337), we obtain the expression

$$
K_{1,2}(\vec{q})=\frac{Z}{2 E} \frac{-1+3\left(1+\frac{Z}{2 E|q|}\right) \frac{q_{3}^{2}}{|\vec{q}|^{2}}}{(|\vec{q}|+Z / E)^{3}}
$$

for the sectional curvature of the Jacobi metric in the 1-2-tangent plane at $\vec{q}$. We can learn several things from that formula:

- Setting $q_{3}=0$ and thus considering planar motion,

$$
K_{1,2}(\vec{q})=-\frac{Z}{2 E(|\vec{q}|+Z / E)^{3}}<0
$$


for positive $E$, and this expression is bounded below, by

$$
K_{1,2}(\vec{q}) \geq-\frac{E^{2}}{2 Z^{2}}
$$

This fact was used extensively in [Kn1], [Kn2] and [KK] to analyze planar motion by going to the smooth branched covering surface

$$
\mathbf{M}:=\left\{(q, Q) \in \mathbb{C} \times \mathbb{C} \mid Q^{2}=\prod_{l=1}^{n}\left(q-s_{l}\right)\right\}
$$

of the configuration plane, equipped with the lifted Jacobi metric (the branched covering being given by projection to the first factor $q$ ).

- For $d=3$ dimensions the sectional curvature (A.2) is neither uniformly bounded in the $\vec{q}$ variable nor definite. In fact, for $\vec{q}=\left(0,0, q_{3}\right)$

$$
K_{1,2}(\vec{q}) \sim \frac{3 E}{4 Z|\vec{q}|} \rightarrow+\infty \quad(q \rightarrow 0) .
$$

We thus consider here geodesic motion in mixed sectional curvature. Although the $E$-dependence of (A.3) is quadratic, whereas (A.4) is only linear in $E$, negative curvature does never dominate positive curvature in our estimates. Namely we have seen in Lemma 9.1 that the minimal distances of the bounded orbits from the nuclei are of the order $1 / E$, so that effectively (A.4), too, goes like $E^{2}$.

Mixed curvature dynamics is rather intractable in general. However, in the case considered here the motion near the singularities can be treated as a perturbation of Keplerian motion, and this allows us to control the motion in the high energy limit.

Whereas the estimates of this papers are somewhat optimal in the high energy limit $E>E_{\text {th }}$, nothing much could be said about the energy region $0<E \leq E_{\text {th }}$ (the exception being Sect. 6).

To the contrary, for two dimensions many results were shown for all positive energies, using the negativity of Gaussian curvature and the topology of the branched covering surface $\mathbf{M}$ (whose fundamental group is non-abelian for $n \geq 3$ ).

The branched covering $\mathbf{M} \rightarrow \mathbb{C}$ globalizes the so-called Levi-Civita transform $Q \mapsto Q^{2}$ of celestial mechanics. So it is natural to pose the question whether there exists a similar globalization of the Hopf map

$$
\mathbb{C}^{2} \rightarrow \mathbb{R}^{3}, \quad z \mapsto\langle z, \vec{\sigma} z\rangle
$$

used in the Kustaanheimo-Stiefel regularization of a 3-dim. Coulomb singularity. As already mentioned, this was done in [HS] for $n=2$ centres. However, a generalization to arbitrary $n$ seems to be unknown.

We expect that the corresponding manifolds, i.e. four-dimensional analogs of Riemann surfaces, should have interesting topological properties.

One last aspect concerns structural stability. Both in $d=2$ and three dimensions the compact set $b_{E} \subset \Sigma_{E}$ of bounded orbits is hyperbolic and thus structurally stable. 
Thus if we continuously move the singularities by suitable maps $S^{1} \rightarrow \mathbb{R}^{d}$, $u \mapsto \vec{s}_{l}(u)$, we obtain a family of Coulombic potentials $V_{u}$ and correspondingly a one-parameter family of bound states $b_{E, u}$. As the parameter varies cyclically, we obtain a permutation of the bounded orbits in $b_{E} \equiv b_{E, 1}$.

For $d=2$ this action of the braid group on $n$ strands of $\mathbb{R}^{2}$ by permutations is nontrivial in general (see Remark 6.12 of [KK]).

Although in $d=3$ dimensions the manifold $\mathcal{N C}$ of non-collinear configurations is not simply connected for $n \geq 3$, the action of the fundamental group $\pi_{1}(\mathcal{N C})$ on $b_{E}$ is trivial.

\section{B. The two-centre problem}

Here we shortly discuss the purely Coulombic two-centre problem, i.e.

$$
V(\vec{q})=\frac{-Z_{1}}{\left|\vec{q}-\vec{s}_{1}\right|}+\frac{-Z_{2}}{\left|\vec{q}-\vec{s}_{2}\right|} .
$$

W.l.o.g. we assume that the two centres are at $\vec{s}_{1}:=\left(\begin{array}{l}1 \\ 0 \\ 0\end{array}\right)$ and $\vec{s}_{2}:=\left(\begin{array}{c}-1 \\ 0 \\ 0\end{array}\right)$. As is well-known (see e.g. Thirring [Th]), the problem is analytically integrable, see $[\mathrm{GKM}]$ for an application to satellite motion and [SR] for an application to semiclassics of the hydrogen molecule.

The motion is integrated using the prolate ellipsoidal coordinates $(\xi, \eta, \varphi) \in$ $\mathbb{R}^{+} \times[0, \pi) \times[0,2 \pi)$ with

$$
\vec{q} \equiv\left(\begin{array}{c}
q_{1} \\
q_{2} \\
q_{3}
\end{array}\right)=\left(\begin{array}{c}
\cosh (\xi) \cos (\eta) \\
\sinh (\xi) \sin (\eta) \cos (\varphi) \\
\sinh (\xi) \sin (\eta) \sin (\varphi)
\end{array}\right) .
$$

As in these coordinates $H$ is independent of $\varphi$, the conjugate momentum

$$
p_{\varphi}=q_{2} p_{3}-q_{3} p_{2}
$$

is a constant of the motion, equal to the first component of angular momentum. We set $l_{1}:=p_{\varphi}\left(x_{0}\right)$ for initial conditions $x_{0}$.

Then by going to extended phase space and using a new time parameter $s$ defined by

$$
\frac{d t}{d s}=2\left(\cosh ^{2}(\xi)-\cos ^{2}(\eta)\right)
$$

the new Hamiltonian function separates:

$$
\mathcal{H}:=\frac{d t}{d s}(H-E)=H_{1}+H_{2}
$$

with

$H_{1}\left(p_{\xi}, \xi\right):=p_{\xi}^{2}+V_{1}(\xi)$ with $\quad V_{1}(\xi):=\frac{l_{1}^{2}}{\sinh ^{2}(\xi)}-2 Z_{+} \cosh (\xi)-2 E \cosh ^{2}(\xi)$ 
$H_{2}\left(p_{\eta}, \eta\right):=p_{\eta}^{2}+V_{2}(\eta) \quad$ with $\quad V_{2}(\eta):=\frac{l_{1}^{2}}{\sin ^{2}(\eta)}+2 Z_{-} \cos (\eta)+2 E \cos ^{2}(\eta)$,

where $Z_{ \pm}:=Z_{2} \pm Z_{1}$.

The motion on $\mathcal{H}^{-1}(0)$ coincides — up to time parameterization — with the motion on $H^{-1}(E)$. Setting

$$
K:=H_{1}\left(x_{0}\right)=-H_{2}\left(x_{0}\right),
$$

we have three generally independent constants of the motion $H, H_{1}$ and $l_{1}$, whose values are denoted by $E, K$ and $l_{1}$, respectively.

The bifurcation set is then given by the set of values for which the mapping from phase space to the constants of the motion is not locally trivial (see [AM], Sect. 4.5). The most interesting subset is the one for $l_{1}=0$, i.e. two-dimensional motion.

By inspection of the extrema of the $V_{i}$ one sees that for $l_{1}=0$ the image of $\left(H, H_{1}\right)$ is the region in $\mathbb{R}^{2}$ bounded by the curves

$$
K_{+}(E):= \begin{cases}Z_{+}^{2} & , 0>E>-Z_{+} / 2 \\ -2\left(Z_{+}+E\right) & , E \leq-Z_{+} / 2\end{cases}
$$

and

$$
K_{-}(E):=\left\{\begin{array}{ll}
\frac{Z_{-}^{2}}{2 E} & , E>\left|Z_{-}\right| / 2 \\
2\left(\left|Z_{-}\right|-E\right) & , E \leq\left|Z_{-}\right| / 2
\end{array} .\right.
$$

The bifurcation diagramme (see Fig. 2) is the union of $K_{-}, K_{+}$, and the lines

$$
E=0 \quad, \quad K=0 \quad \text { and } \quad K_{0}(E):=-2\left(Z_{+}+E\right)
$$

inside the image of $\left(H, H_{1}\right)$.

The line $K_{0}$ corresponds to the $\left(H, H_{1}\right)$-values of the closed orbit wandering between the centres $\vec{s}_{1}$ and $\vec{s}_{2}$, i.e. having coordinate $\xi=0$.

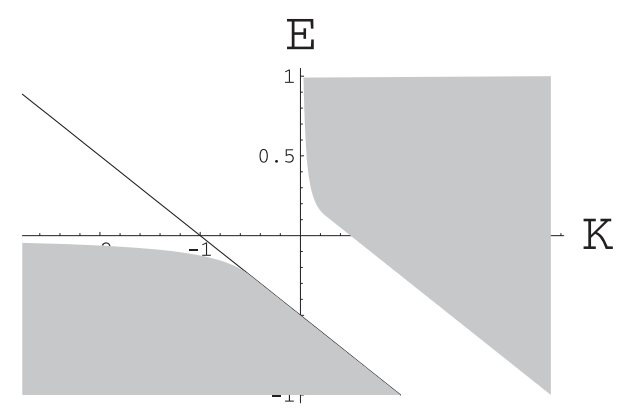

Fig. 2. Bifurcation diagramme

The following relations are useful for the scattering problem. In the (1-2)-plane, i.e. for $\varphi=0$, we have the polar coordinates $q_{1}=r \cos \phi, q_{2}=r \sin \phi$. Then 
$r^{2}=\cosh ^{2}(\xi)-\sin ^{2}(\eta)$ and $\tan (\phi)=\tanh (\xi) \tan (\eta)$ so that in the $r \rightarrow \infty$ limit $\eta$ coincides with $\phi$.

In the same limit $p_{\eta}=q_{1} p_{2}-q_{2} p_{1}-e^{-\xi}\left(p_{1} \sin \eta+p_{2} \cos (\eta)\right)$ coincides with the angular momentum $q_{1} p_{2}-q_{2} p_{1}$ of the (1-2)-plane.

This suffices to relate the asymptotic data $\left(p_{\eta}^{ \pm}, \eta^{ \pm}\right):=\lim _{s \rightarrow \vec{s}^{ \pm}}\left(p_{\eta}(s), \eta(s)\right)$ with the ones in the original system (the times $s^{+}>s^{-}$being defined by $\left.\lim _{s \rightarrow \vec{s}^{ \pm}} \xi(s)=\infty\right)$. The constant $K$ is then given by $\left(p_{\eta}^{ \pm}\right)^{2}+V_{2}\left(\eta^{ \pm}\right)$. Equalling the elliptic integrals

$$
s^{+}-s^{-}=\left|\int_{\eta^{-}}^{\eta^{+}} \frac{d \eta}{\sqrt{-K-V_{2}(\eta)}}\right|
$$

respectively

$s^{+}-s^{-}=2 \int_{\xi_{\min }}^{\infty} \frac{d \xi}{\sqrt{K-V_{1}(\xi)}} \quad$ with $\quad \cosh \left(\xi_{\min }\right)=\frac{-Z_{+}}{2 E}+\sqrt{\left(\frac{Z_{+}}{2 E}\right)^{2}-\frac{K}{2 E}}$

then suffice in principle to calculate analytically the scattering transformation, but the expressions become rather lengthy.

Acknowledgements. I thank the Max Planck Institute for Mathematics in the Sciences (Leipzig), where a part of this paper was written, for hospitality and support.

\section{References}

[AaMi] Aarseth, S., Mikkola, S.: An Implementation of $N$-body Chain Regularization. Celestial Mechanics and Dynamical Astronomy 57, 439-459 (1993)

[Ag] Agmon, S.: Lectures on Exponential Decay of Solutions of Second-Order Elliptic Equations. Princeton: Princeton University Press 1983

[AM] Abraham, R., Marsden, J.E.: Foundations of Mechanics. Reading: Benjamin 1978

[Bo] Bolotin, S.V.: Nonintegrability of the $n$-centre problem for $n>2$. Vestnik Mosk. Gos. Univers., ser. math. mekh. 46 (1982), No. 4

[BT] Bolsinov, A.V.; Taimanov, I.A.: Integrable geodesic flows with positive topological entropy. Invent. math. 140, 639-650 (2000)

[DG] Dereziński, J., Gérard, C.: Scattering Theory of Classical and Quantum $N$ Particle Systems. Texts and Monographs in Physics. Berlin: Springer 1997

[DK] Dierkes, T., Knauf, A.: Perturbations of the planar $n$-centre problem. In preparation

[Du] Duistermaat, J.J.: On the Morse Index in Variational Calculus. Adv. Math. 21, 173-195 (1976)

[Ec] Eckhardt, B.: Irregular Scattering. Physica D 33, 89-98 (1988)

[EJ] Eckhardt, B., Jung, Ch.: Regular and irregular potential scattering. J. Phys. A: Math. Gen. 19 L829-L833 (1986)

[Fa] Falconer, K.: Techniques in Fractal Geometry. New York: Wiley 1997

[Ga] Gaspard, P.: Chaos, Scattering and Statistical Mechanics. Cambridge: Cambridge University Press 1998

[GK] Gérard, C., Knauf, A.: Collisions for the Quantum Coulomb Hamiltonian. Commun. Math. Phys. 143, 17-26 (1991) 
[GKM] Golin, S., Knauf, A., Marmi, S.: The Hannay Angles: Geometry, Adiabaticity, and an Example. Commun. Math. Phys. 123, 95-122 (1989)

[HKSW] Helffer, B., Siedentop, H., Knauf, A., Weikard, R.: On the Absence of a First Order Correction for the Number of Bound States of a Schrödinger Operator with Coulomb Singularity. Commun. P.D.E. 17, 615-639 (1992)

[HS] Helffer, B., Siedentop, H.: A generalization of the Kustaanheimo-Stiefel transform for two-centre systems. J. Bull. Lond. Math. Soc. 28, 33-42 (1996)

[Hi] Hirsch, M.: Differential Topology. Graduate Texts in Mathematics, Vol. 33. Berlin, Heidelberg, New York: Springer 1988

[Hu Hunziker, W.: Scattering in Classical Mechanics. In: Scattering Theory in Mathematical Physics. J.A. La Vita, J.-P. Marchand, eds., Dordrecht: Reidel 1974

[KH] Katok, A., Hasselblatt, B.: Modern Theory of Dynamical Systems. Cambridge: Cambridge University Press 1997

[KK] Klein, M., Knauf, A.: Classical Planar Scattering by Coulombic Potentials. Lecture Notes in Physics m 13. Berlin, Heidelberg, New York: Springer 1992

[K1] Klingenberg, W.: Riemannian Geometry. Studies in Mathematics 1; Berlin, New York: De Gruyter 1995

[Kn1] Knauf, A.: Ergodic and Topological Properties of Coulombic Periodic Potentials. Commun. Math. Phys. 110, 89-112 (1987)

[Kn2] Knauf, A.: Coulombic Periodic Potentials: The Quantum Case. Annals of Physics 191, 205-240 (1989)

[Kn3] Knauf, A.: Qualitative Aspects of Classical Potential Scattering. Regular and Chaotic Dynamics 4, 1-20 (1999)

[KR] Koecher, M., Remmert, R.: Hamiltonsche Quaternionen. In: Ebbinghaus, H.-D. et al: Zahlen. Berlin, Heidelberg, New York: Springer 1992

[Ku] Kummer, M.: On the Regularization of the Kepler Problem. Commun. Math. Phys. 84, 133-152 (1982)

[KS] Kustaanheimo, P., Stiefel, E.: Perturbation theory of Kepler motion based on spinor regularization. Journal für Mathematik 218, 204-219 (1965)

[Ma] Mattila, P.: Geometry of Sets and Measures in Euclidean Spaces. Cambridge studies in advanced mathematics, Vol. 44. Cambridge: Cambridge University Press 1995

[DS] McDuff, D., Salamon, D.: Introduction to Symplectic Topology. Oxford: Oxford University Press 1995

[Mi] Milnor, J.: Morse Theory. Annals of Mathematics Studies. Princeton: Princeton University Press 1973

[Mo] Moser, J.: Regularization of Kepler's Problem and the Averaging Method on a Manifold. Commun. Pure Appl. Math. 23, 609-636 (1970)

[PP] Parry, W., Pollicott, M.: An analogue of the prime number theorem for closed orbits of Axiom A flows. Ann. Math. 118, 573-591 (1983)

[Ru] Ruelle, D.: Thermodynamic Formalism. Reading: Addison-Wesley 1978

[Sim] Simon, B.: Wave Operators for Classical Particle Scattering. Commun. Math. Phys. 23, 37-49 (1971)

[Sin] Sinai, Ya.G., ed.: Dynamical Systems II. Encyclopaedia of Mathematical Sciences, Vol. 2. Berlin, Heidelberg, New York: Springer 1989

[Sm] Smilansky, U.: The Classical and Quantum Theory of Chaotic Scattering. Lecture Notes Summer School on 'Quantum Chaos'. Les Houches 1989

[Sp] Spivak, M.: Differential Geometry, Vol. II. Berkeley: Publish or Perish 1979

[StS] Stiefel, E.L., Scheifele, G.: Linear and Regular Celestial Mechanics. Berlin: Springer 1971

[SR] Strand, M.P., Reinhardt, W.P.: Semiclassical quantization of the low lying electronic states of $H_{2}^{+}$. J. Chem. Phys. 70, 3812-3827 (1979)

[Te] Tél, T.: Transient Chaos. In: Directions in Chaos, Vol. 4, ed. Hao Bai-lin. Singapore: World Scientific 1990

[Th] Thirring, W.: Lehrbuch der Mathematischen Physik 1. 2nd ed.; Wien, New York: Springer 1988 
[Tr] Tricot, C., Jr: Two definitions of fractional dimension. Math. Proc. Camb. Phil. Soc. 92, 57-74 (1982)

[Vi] Vinti, J.P.: New Method of Solution for Unretarded Satellite Orbits. J. Res. Nat. Bur. Standards 63, 105-116 (1959)

[Wa] Walters, P.: An Introduction to Ergodic Theory. Graduate Texts in Mathematics, Vol. 79. Berlin, Heidelberg, New York: Springer 1982

Note added in proof. The anonymous referee informed me about the interesting related article: S.V. Bolotin, P. Negrini: Regularization and topological entropy for the spatial $n$ center problem, which meanwhile appeared in Ergodic Theory and Dynamical Systems 21, 383-399 (2001). Concerning the context of the $n$-centre-problem on $\mathbb{R}^{3}$, the authors succeed to construct a global regularization of $n$ attracting singularities, based on the local KS transform. Furthermore, they prove that for $n \geq 3$ the topological entropy is strictly positive for all energies $E \geq 0$, whereas the present paper is only dealing with all energies above a positive threshold energy. Additionally, the authors prove several results for configuration manifolds different from $\mathbb{R}^{3}$. I do not believe, however, that these topological methods could be used to substantially simplify the proofs of the analytic results given in the present paper. 